\title{
Characterization of Extremal KMS States on Groupoid C*-Algebras
}

\author{
Rafael Pereira Lima \\ DisSERTAÇÃO APRESENTADA \\ aO Instituto de Matemática e Estatística \\ DA \\ UniversidAdE DE SÃO PAUlO \\ PARA \\ OBTENÇÃO DO TÍTULO \\ $\mathrm{DE}$ \\ Mestre em CiênCIAS
}

Programa: Mestrado em Matemática Aplicada

Orientador: Prof. Dr. Rodrigo Bissacot

Durante o desenvolvimento deste trabalho o autor recebeu auxílio financeiro do $\mathrm{CNPq}$

São Paulo, julho de 2019 


\section{Characterization of Extremal KMS States on Groupoid C*-Algebras}

Esta dissertação contém as correções e alterações sugeridas pela Comissão Julgadora durante a defesa

realizada por Rafael Pereira Lima em 1/7/2019.

O original encontra-se disponível no Instituto de Matemática e Estatística da Universidade de São Paulo.

Comissão Julgadora:

- Prof. Dr. Cristian Ortiz (presidente) - IME-USP

- Prof. Dr. Alcides Buss - UFSC

- Prof. Dr. Alexandre Baraviera - UFRGS 


\section{Agradecimentos}

Consegui escrever esta dissertação por causa da ajuda de algumas pessoas. Gostaria de agradecer aos meus pais, minha irmã e minha madrinha pelo incentivo que sempre tive.

Sou grato pela ótima orientação do professor Rodrigo Bissacot, que me preparou para a pesquisa e se preocupa com a carreira dos alunos, além de ser um exemplo para mim. Aos colegas do grupo pela ajuda durante o desenvolvimento da dissertação, principalmente Lucas, João, Rodrigo e Thiago, que revisaram o trabalho durante vários seminários. Em particular, gostaria de agradecer ao Lucas, por me recomendar para o professor Rodrigo.

Agradeço ao professor Severino Toscano pela ajuda durante o mestrado. Especialmente, sou muito grato pela ajuda e apoio do professor Paulo Cordaro, o que foi fundamental para eu conseguir terminar o mestrado. 


\section{Resumo}

Nesta dissertação de mestrado, estudamos um teorema de Neshveyev [17] que descreve todos os estados KMS em uma $\mathrm{C}^{*}$-álgebra de um grupóide étale localmente compacto Hausdorff satisfazendo o segundo axioma de enumerabilidade. Depois estudamos um resultado provado por Thomsen [26] que caracteriza os estados KMS extremais nessa $\mathrm{C}^{*}$-álgebra para um grupóide de Renault-Deaconu.

Palavras-chave: $\mathrm{C}^{*}$-álgebras, estados KMS, medidas conformes, grupóides. 


\section{Abstract}

In this master's thesis we study a theorem due to Neshveyev [17] which describes all KMS states on the groupoid $\mathrm{C}^{*}$-algebra for a locally compact Hausdorff second countable étale groupoid. Then we study a result due to Thomsen [26] which characterizes the extremal KMS states on this $\mathrm{C}^{*}$-algebra for a Renault-Deaconu groupoid.

Keyworkds: $C^{*}$-algebras, KMS states, conformal measures, groupoids. 


\section{Contents}

\begin{tabular}{lll}
\hline Introduction & 6 \\
\hline
\end{tabular}

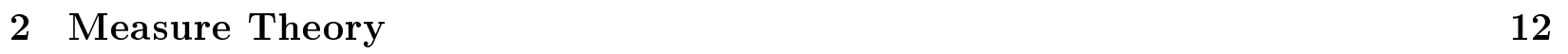

2.1 Radon-Nikodym Theorem . . . . . . . . . . . . . . . . 12

2.2 Pushforward Measure . . . . . . . . . . . . . . . . . . . . . 15

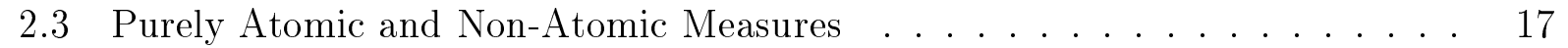

2.4 Measures on Locally Compact Spaces . . . . . . . . . . . . . . . . 18

$2.5 \quad \mu$-Measurable Functions $\ldots \ldots \ldots \ldots$

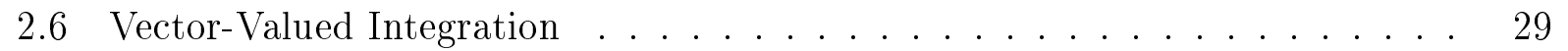

3 Groupoids 41

3.1 Introduction $\ldots \ldots \ldots \ldots \ldots \ldots \ldots$

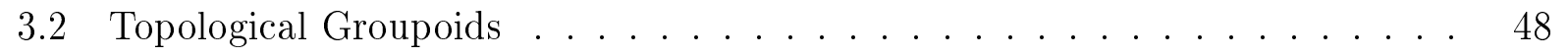

3.3 Groupoid $\mathrm{C}^{*}$-Algebras $\ldots \ldots \ldots \ldots \ldots \ldots$

4 Renault's Disintegration Theorem

4.1 Haar Systems . . . . . . . . . . . . . . . . 74

4.2 Borel Hilbert Bundles . . . . . . . . . . . . . . . . . . . . . . . . 82

4.3 Renault's Disintegration Theorem . . . . . . . . . . . . . . . . 92 
5 Neshveyev's Theorems

5.1 KMS States . . . . . . . . . . . . . . . . . . . . 95

5.2 First Theorem . . . . . . . . . . . . . . . . 124

5.3 Second Theorem . . . . . . . . . . . . . . . . . . 140

6 Renault-Deaconu Groupoid

6.1 Introduction . . . . . . . . . . . . . . . . . . 159

6.2 Full orbits . . . . . . . . . . . . . . . . . . 171

6.3 Conformal Measures $\ldots \ldots \ldots \ldots$. . . . . . . . . . . . . . . . . . .

6.4 KMS States on the Renault-Deaconu Groupoid . . . . . . . . . . . . . 201

\begin{tabular}{|lll}
\hline Concluding Remarks & 214 \\
\hline
\end{tabular} 


\section{Chapter 1}

\section{Introduction}

The purpose of this thesis is to find all KMS states on groupoid $\mathrm{C}^{*}$-algebras when the groupoid satisfies certain topological conditions. This result was proved by Neshveyev in [17]. Later we study a theorem due to Thomsen [26] which applies Neshveyev's theorem to a Renault-Deaconu groupoid to characterize its extremal KMS states.

Groupoids are a generalization of groups where not every pair of elements can be multiplied but each element has an inverse. This structure can be seen as a collection of arrows attached to points on a plane, as shown in Figure 1.1. Such arrows can be composed if the end (called range) of the first arrow is the source of the second. The inverse is obtained by reversing the direction of the arrow and each point is identified with an element of the groupoid assuming its corresponding vector is the null vector.

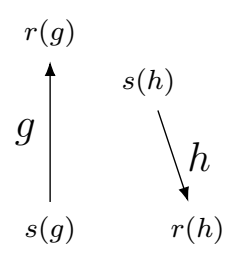

(a)

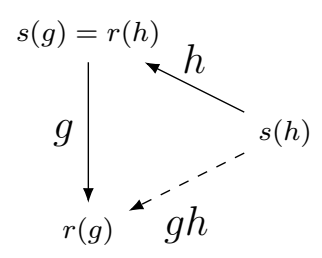

(b)

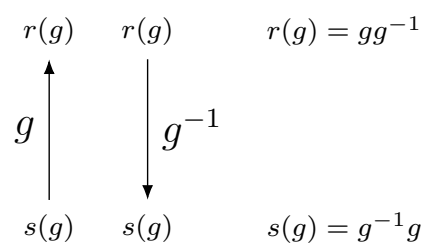

(c)

Figure 1.1: Groupoids can be seen as arrows on a plane. $s(g)$ and $r(g)$ denote the source and range of $g$. (a) $g$ and $h$ are not composable, since $s(g) \neq r(h)$; (b) The composition of $g$ and $h$ is $g h$; (c) $g^{-1}$ is the inverse of $g$. Note that $g^{-1} g=s(g)$ and $g g^{-1}=r(g)$. 
Given a groupoid $G, G^{(2)}$ is the set of composable elements. It consists of all pairs of elements in $G$ which can be multiplied. $G^{(0)} \subset G$ is the set of units. $G$ is endowed with the multiplication (also called composition) and inversion operations. $r, s: G \rightarrow G^{(0)}$ are the range and source maps. Later we will define formally the notion of groupoids. The results on groupoids in this thesis can be found in Rodrigo Frausino's thesis [9]. In fact, this thesis can be seen as a sequel of his work because he also describes groupoid $\mathrm{C}^{*}$-algebras and the Renault-Deaconu groupoid. In addition, many results here are based on his work.

Under certain conditions, we can equip the groupoid with a topology in such a way that $r, s$ are local homeomorphisms and the sets $G_{x}^{x}=s^{-1}(x) \cap r^{-1}(x)$ are discrete and countable groups, and we assume this topology satisfies other conditions. In this case, we can equip the space of continuous and compactly supported functions on $G$, denoted by $C_{c}(G)$, with an involution and a convolution which is not the pointwise multiplication. Then $C_{c}(G)$ becomes a $*$-algebra, not necessarily commutative.

In order to define the groupoid $\mathrm{C}^{*}$-algebra $C^{*}(G)$, we equip $C_{c}(G)$ with a norm which depends on the $*$-representations of $C_{c}(G)$. Then $C^{*}(G)$ is defined as the completion of $C_{c}(G)$ with respect to this norm.

Let $c$ be a continuous $\mathbb{R}$-valued 1-cocycle, that is, a continuous function $c: G \rightarrow \mathbb{R}$ such that $c\left(g_{1} g_{2}\right)=c\left(g_{1}\right)+c\left(g_{2}\right)$ for $\left(g_{1}, g_{2}\right) \in G^{(2)}$. Then we fix a dynamics on $C^{*}(G)$ defined by $\tau_{t}(f)(g)=e^{i t c(g)} f(g)$ for every $f \in C_{c}(G), g \in G$ and $t \in \mathbb{R}$. For $f \in C_{c}(G)$, we can extend the definition of $\tau$ to complex parameters, that is, $\tau_{z}(f)$ is well-defined. Given $\beta \in \mathbb{R}$, we say that a state $\varphi$ on $C^{*}(G)$ is a KMS state if $\varphi\left(f_{1} \tau_{i \beta}\left(f_{2}\right)\right)=\varphi\left(f_{2} f_{1}\right)$ for every $f_{1}, f_{2} \in C_{c}(G)$.

KMS states characterizes the equilibrium states in quantum statistical mechanics. A theorem due to Neshveyev describes every KMS state $\varphi$ on $C^{*}(G)$ by an explicit formula. In fact, there is a correspondence between $\varphi$ and a pair $\left(\mu,\left\{\varphi_{x}\right\}_{x \in G^{(0)}}\right)$ satisfying some conditions, such that $\mu$ is a probability measure on $G^{(0)}$ and each $\varphi_{x}$ is a state on $C^{*}\left(G_{x}^{x}\right)$. An important step in the proof of this theorem is the Renault's disintegration theorem [15], which will be used to obtain $\left\{\varphi_{x}\right\}_{x \in G^{(0)}}$ and $\mu$ when a KMS state $\varphi$ on $C^{*}(G)$ is given.

In the final part of the thesis, we define the Renault-Deaconu groupoid and prove Thom- 
sen's theorem.

Let $X$ be a locally compact, second countable, locally Hausdorff space. Given $\sigma: X \rightarrow X$ a local homeomorphism, the Renault-Deaconu groupoid is defined by

$$
\mathcal{G}=\left\{(x, k, y): k=n-m, \sigma^{n}(x)=\sigma^{m}(y)\right\}
$$

with composition $(x, k, y)(y, l, z)=(x, k+l, z)$ and inversion $(x, k, y)^{-1}=(y,-k, x)$.

Although the definition of $\mathcal{G}$ is abstract, it is useful to have an intuition about this structure. Note that the sequence $\left\{\sigma^{n}(x)\right\}_{n \in \mathbb{N}}$ can be seen as a trajectory starting at $x$. Given $y \in X,(x, k, y) \in \mathcal{G}$ means that the trajectories of $x$ and $y$ eventually meet. $k$ can be interpreted as the delay of one trajectory with respect to the other. Figure 1.2 shows this idea.

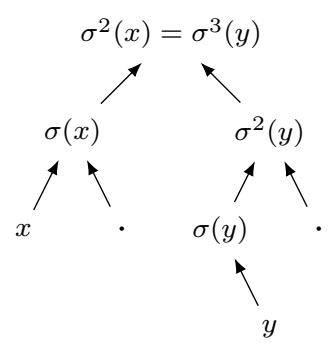

Figure 1.2: If $(x, k, y) \in \mathcal{G}$ then the trajectories $\left\{\sigma^{l}(x)\right\}_{l \in \mathbb{N}}$ and $\left\{\sigma^{l}(y)\right\}_{l \in \mathbb{N}}$ eventually meet. $k$ can be seen as the delay of one trajectory with respect to the other. In this figure, $k=-1$, since $\sigma^{2}(x)=\sigma^{3}(y)$.

Given a continuous function $F: X \rightarrow \mathbb{R}$, we can define a continuous $\mathbb{R}$-valued 1-cocycle $c_{F}$ by

$$
c_{F}(x, k, y)=\sum_{j=0}^{n-1} F\left(\sigma^{j}(x)\right)-\sum_{j=0}^{m-1} F\left(\sigma^{j}(y)\right)
$$

for $n, m \in \mathbb{N}$ such that $k=n-m$ and $\sigma^{n}(x)=\sigma^{m}(y)$. In fact, there exists a bijection between $\mathbb{R}$-valued 1-cocycles on $\mathcal{G}$ and continuous real-valued functions on $X$. Then we define the dynamics on $C^{*}(\mathcal{G})$ by $\tau_{t}(f)(g)=e^{i t c_{F}(g)} f(g)$. We want to describe the KMS 
states on $C^{*}(G)$ with respect to this dynamics.

Since extremal KMS states are sufficient to describe all KMS states on a $\mathrm{C}^{*}$-algebra, Thomsen's theorem characterizes only the extremal KMS states on the full $\mathrm{C}^{*}$-algebra of this groupoid. In this case, we show that the probability measures corresponding to the KMS states are $e^{\beta F}$-conformal measures on $X$.

The orbit $\mathcal{O}(x)$ of $x$ denotes the set of points $y \in X$ such that $(x, k, y) \in \mathcal{G}$ for some $k$. There is a bijection between orbits in $X$ and the set of extremal atomic $e^{\beta F}$-conformal probability measures on $X$. Thomsen's theorem divides extremal KMS-states $\varphi$ corresponding to measures $m$ in three cases:

- when $m$ is continuous;

- when $m$ purely atomic and corresponds to a periodic orbit;

- when $m$ purely atomic and corresponds to an aperiodic orbit.

In each case the theorem gives a formula for $\varphi$.

This thesis is structured in the following way:

Chapter 2, we recall some concepts of measure theory. This chapter is important to understand the properties of the measures corresponding to KMS states on grupoid $\mathrm{C}^{*}$ algebras. We also define the integral of vector-valued functions on a Banach space.

Chapter 3; we define groupoids and topological groupoids. Then we define the groupoid $\mathrm{C}^{*}$-algebra and prove some properties of this $\mathrm{C}^{*}$-algebra.

Chapter 4 4 we define concepts necessary to understand Renault's disintegration theorem and we state this theorem. However, we do not prove this result.

Chapter 5. we define KMS states on arbitrary $\mathrm{C}^{*}$-algebras and prove some properties. Then we prove two theorems due to Neshveyev, used to describe KMS states on some groupoid $\mathrm{C}^{*}$-algebras. We state these theorems below and we refer to them as Neshveyev's first theorem and Neshveyev's second theorem, respectively. 
Theorem. [17, Theorem 1.1] Let $G$ be a locally compact Hausdorff second countable étale groupoid. There is a one-to-one correspondence between states on $C^{*}(G)$ with centralizer containing $C_{0}\left(G^{(0)}\right)$ and pairs $\left(\mu,\left\{\varphi_{x}\right\}_{x}\right)$ consisting of a probability measure $\mu$ on $G^{(0)}$ and a $\mu$-measurable field of states $\varphi_{x}$ on $C^{*}\left(G_{x}^{x}\right)$. Namely, the state corresponding to $\left(\mu,\left\{\varphi_{x}\right\}_{x}\right)$ is given by

$$
\varphi(f)=\int_{G^{(0)}} \sum_{g \in G_{x}^{x}} f(g) \varphi_{x}\left(u_{g}\right) d \mu(x) \quad \text { for } \quad f \in C_{c}(G)
$$

Theorem. [17, Theorem 1.3] Let $G$ be a locally compact second countable Hausdorff étale groupoid. Let $c$ be a continuous $\mathbb{R}$-valued 1-cocycle on $G$ and $\tau$ be the dynamics on $C^{*}(G)$ defined by $\tau_{t}(f)(g)=e^{i t c(g)} f(g)$ for $f \in C_{c}(G), g \in G$. Fix $\beta \in \mathbb{R}$. Then there exists a oneto-one correspondence between $\mathrm{KMS}_{\beta}$-states on $C^{*}(G)$ and pairs $\left(\mu,\left\{\varphi_{x}\right\}_{x \in G^{(0)}}\right)$ consisting of a probability measure $\mu$ on $G^{(0)}$ and a $\mu$-measurable field of states $\varphi_{x}$ on $C^{*}\left(G_{x}^{x}\right)$ such that:

(i) $\mu$ is quasi-invariant with Radon-Nikodym derivative $e^{-\beta c}$;

(ii) $\varphi_{x}\left(u_{g}\right)=\varphi_{r(h)}\left(u_{h g h^{-1}}\right)$ for every $g \in G_{x}^{x}$ and $h \in G_{x}$, for $\mu$-a.e. $x$; in particular, $\varphi_{x}$ is tracial for $\mu$-a.e. $x$;

(iii) $\varphi_{x}\left(u_{g}\right)=0$ for all $g \in G_{x}^{x} \backslash c^{-1}(0)$, for $\mu$-a.e. $x$.

Chapter 6, we define the Renault-Deaconu groupoid, describe some of its properties, then we characterize the extremal KMS-states proving the following theorem due to Thomsen:

Theorem. [26, Theorem 2.2] Let $\beta \in \mathbb{R} \backslash\{0\}$. Assume that the periodic points of $\sigma$ are countable. The extremal $\mathrm{KMS}_{\beta}$-states for $\tau$ are

1. States $\phi_{m}$, where $m$ is an extremal and continuous (non-atomic) $e^{\beta F}$-conformal Borel probability measure on $X$; 
2. The states $\phi_{x}^{\lambda}$, where $\lambda \in \mathbb{C},|\lambda|=1$ and $x$ is periodic with minimum period $p$, such that

$$
\sum_{j=0}^{p-1} F\left(\sigma^{j}(x)\right)=0 \quad \text { and } \quad \sum_{n=1}^{\infty} \sum_{y \in Y_{n}} \exp \left(-\beta \sum_{j=0}^{n-1} F\left(\sigma^{j}(y)\right)\right)<\infty
$$

3. The states $\phi_{m_{z}}$ where $z$ is aperiodic and $\beta$-summable. 


\section{Chapter 2}

\section{Measure Theory}

The main theorems in this thesis, described in Chapters 5 and 6, shows that there is a relation between a KMS state on a particular groupoid $\mathrm{C}^{*}$-algebra and a probability measure on a subset of this groupoid. In order to understand these theorems, we should recall some results from measure theory. We also generalize the notion of integral to functions from a measurable space to a Banach space.

\subsection{Radon-Nikodym Theorem}

The Radon-Nikodym theorem proves that, under certain conditions, two measures $\nu, \mu$ are related by a non-negative measurable function $f$, denoted the Radon-Nikodym derivative. In this case, $\nu$ can be interpreted as the integral of $f$ with respect to $\mu$. The results in this section can be found in [14].

Definition 2.1.1. Let $X$ be a measurable space and let $\mu, \nu$ be measures on $X$. We say $\nu$ is absolutely continuous with respect to $\mu$ if

$$
\mu(A)=0 \quad \text { implies } \quad \nu(A)=0, \quad A \text { measurable. }
$$

We denote $\nu \ll \mu$. 
Note that $\ll$ defines a partial order on the set of measures on $X$ (assuming the $\sigma$-algebra is fixed.)

Theorem 2.1.2. (Radon-Nikodym Theorem) Let $X$ be a measurable space and $\nu, \mu$ be $\sigma$-finite measures on $X$. If $\nu \ll \mu$ then there exists a measurable nonnegative function $f$ on $X$ such that $f$ is finite $\mu$-a.e. and

$$
\nu(A)=\int_{A} f d \mu, \quad A \subset X \text { measurable. }
$$

Moreover, $\nu$ is finite if and only if $f$ is integrable.

The function $f$ in Theorem 2.1.2 is called the Radon-Nikodym derivative of $\nu$ with respect to $\mu$ and is denoted by

$$
f=\frac{d \nu}{d \mu}
$$

Although we write 2.1 as an equality, the function $f$ is not unique. If there exists a function $g$ satisfying 2.1, then $f=g \mu$-a.e. We assume equality since we can neglect values of $f$ on a null set.

Remark 2.1.3. If the measure space $X$ is locally compact Hausdorff, the Radon-Nikodym derivative is a local property. That is, if we want to find the Radon-Nikodym derivative $\frac{d \nu}{d \mu}(x)$ on a neighborhood of a point $x$, it is sufficient to study the relation between $\nu, \mu$ on this neighborhood.

In fact, let $U$ be an open neighborhood of $x$ and assume there exists a measurable function $\Delta$ on $U$ such that

$$
\int_{U} f(y) d \nu(y)=\int_{U} f(y) \Delta(y) d \mu(y)
$$


for every $f \in C_{c}(U)$. Then using the definition of $d \nu / d \mu$, we have

$$
\int_{U} f(y) \frac{d \nu}{d \mu}(y) d \mu(y)=\int_{U} f(y) \Delta(y) d \mu(y) .
$$

Since $f$ is arbitrary, we have

$$
\frac{d \nu}{d \mu}(y)=\Delta(y), \quad \text { for } \mu \text {-a.e. } y \in U \text {. }
$$

Example 2.1.4. Let $\mu$ be the Lebesgue measure on $\mathbb{R}$. Define the measure $\nu$ on $\mathbb{R}$ by $\nu([a, b])=a^{3}-b^{3}$, for every closed interval $[a, b]$. Then

$$
\nu([a, b])=\int_{a}^{b} 3 x^{2} \mu(x) .
$$

Then we have, by Remark 2.1.3.

$$
\frac{d \nu}{d \mu}(x)=3 x^{2}
$$

Now we state some results on the Radon-Nikodym derivative which will be used throughout the thesis.

Proposition 2.1.5. Let $\mu, \nu$ be $\sigma$-finite measures on $X$ such that $\nu \ll \mu$. Then, for every integrable function $g$ with respect to $\nu$ we have

$$
\int_{X} g d \nu=\int_{X} g \frac{d \nu}{d \mu} d \mu
$$

Proposition 2.1.6. If $\mu, \nu$ are $\sigma$-finite measures on $X$ such that $\nu \ll \mu$ and $d \nu / d \mu \neq 0$ $\mu$-a.e., then $\mu \ll \nu$ and

$$
\frac{d \mu}{d \nu}=\left(\frac{d \nu}{d \mu}\right)^{-1}
$$


Proposition 2.1.7. (Chain rule) If $\mu, \nu, \eta$ are measures on $X$ satisfying $\eta \ll \nu \ll \mu$, then

$$
\frac{d \eta}{d \mu}=\frac{d \eta}{d \nu} \frac{d \nu}{d \mu}
$$

\subsection{Pushforward Measure}

Given a measurable function $T: X \rightarrow Y$ between two measurable spaces, assume $X$ is endowed with a measure $\mu$. Then we can define a measure on $Y$, referred to as the pushforward measure. This notion is defined in [24].

This notion will be used to prove Theorem 6.3.21 on page 194 .

Theorem. Let $\beta \in \mathbb{R}$. A measure $\mu$ on $\mathcal{G}^{(0)}$ is $e^{\beta F}$-conformal if, and only if, $\mu$ is quasiinvariant with Radon-Nikodym derivative $e^{-\beta c_{F}}$.

This theorem shows that one of the hypothesis of Neshveyev's second theorem holds for every $e^{\beta F}$-conformal measure on the unit space of the Renault-Deaconu groupoid. This will be used to prove Thomsen's theorem.

Definition 2.2.1. Let $X, Y$ be measurable spaces. Let $\mu$ be a measure on $X$. Given a measurable function $\sigma: X \rightarrow Y$, we define the pushforward measure $\sigma_{*} \mu$ on $Y$ by

$$
\int_{Y} f d\left(\sigma_{*} \mu\right)=\int_{X} f \circ \sigma d \mu .
$$

Lemma 2.2.2. Equation 2.2 is equivalent to

$$
\sigma_{*} \mu(A)=\mu\left(\sigma^{-1}(A)\right), \quad \text { for every } A \subset Y \text { measurable. }
$$

Proof. Assume (2.2) holds. Let $A \subset Y$ be measurable. Then $\chi_{A}$ is a measurable function 
on $Y . \sigma$ is measurable, then $\chi_{A} \circ \sigma$ is measurable on $X$. Note that, for $x \in A$,

$$
\chi_{A} \circ \sigma(x)=\left\{\begin{array}{ll}
1 & \text { if } \sigma(x) \in A \\
0 & \text { otherwise }
\end{array}=\left\{\begin{array}{ll}
1 & \text { if } x \in \sigma^{-1}(A) \\
0 & \text { otherwise }
\end{array}=\chi_{\sigma^{-1}(A)} .\right.\right.
$$

Hence,

$$
\sigma_{*} \mu(A)=\int_{Y} \chi_{A}(y) d\left(\sigma_{*} \mu\right)=\int_{X} \chi_{A} \circ \sigma(x) d \mu(x)=\int_{X} \chi_{\sigma^{-1}(A)} d \mu(x)=\mu\left(\sigma^{-1}(A)\right)
$$

Conversely, suppose 2.3 holds. Let $\varphi$ be a simple nonnegative measurable function on $Y$. There exist $a_{1}, \ldots, a_{n} \geq 0, A_{1}, \ldots, A_{n}$ measurable on $Y$ such that $\varphi=\sum_{i=1}^{n} a_{i} \chi_{A_{i}}$. Then

$$
\begin{aligned}
\int_{Y} \varphi d \sigma_{*} \mu & =\sum_{i=1}^{n} a_{i} \sigma_{*} \mu\left(A_{i}\right)=\sum_{i=1}^{n} a_{i} \mu\left(\sigma^{-1}\left(A_{i}\right)\right) \\
& =\sum_{i=1}^{n} a_{i} \int_{X} \chi_{\sigma^{-1}\left(A_{i}\right)}(x) d \mu(x) \\
& =\sum_{i=1}^{n} a_{i} \int_{X} \chi_{A_{i}} \circ \sigma(x) d \mu(x) \\
& =\int_{X} \varphi \circ \sigma(x) d \mu(x) .
\end{aligned}
$$

Let $f$ be a measurable function on $Y$. Assume $f$ is nonnegative. Then there exists a sequence of simple nonnegative functions bounded by $f$ and converging to $f$. Hence 2.2 holds for $f$. Therefore (2.2) holds for every measurable function on $Y$.

Lemma 2.2.3. Let $\mu$ be a measure on $X$. Let $\sigma_{2}: X \rightarrow Y, \sigma_{1}: Y \rightarrow Z$ be measurable. Then $\sigma_{1 *} \sigma_{2 *} \mu=\left(\sigma_{1} \circ \sigma_{2}\right)_{*} \mu$.

Proof. Let $A \subset X$ be measurable. Then,

$$
\begin{aligned}
\sigma_{1 *} \sigma_{2 *} \mu(A) & =\sigma_{1 *}\left(\sigma_{2 *} \mu\right)(A)=\sigma_{2 *} \mu\left(\sigma_{1}^{-1}(A)\right) \\
& =\mu\left(\sigma_{2}^{-1}\left(\sigma_{1}^{-1}(A)\right)\right)=\mu\left(\left(\sigma_{1} \circ \sigma_{2}\right)^{-1}(A)\right)
\end{aligned}
$$




$$
=\left(\sigma_{1} \circ \sigma_{2}\right)_{*}(A)
$$

Lemma 2.2.4. Let $\mu, \nu$ be measures on $X$ such that $\nu \ll \mu$. Let $\sigma: X \rightarrow Y$ be a measurable bijection such that $\sigma^{-1}$ is measurable. Then $\sigma_{*} \nu \ll \sigma_{*} \mu$ and

$$
\frac{d \sigma_{*} \nu}{d \sigma_{*} \mu}(y)=\frac{d \nu}{d \mu}\left(\sigma^{-1}(y)\right) \quad y \in Y .
$$

Proof. Let $f$ be a $\sigma_{*} \nu$-integrable function on $Y$. Then

$$
\begin{aligned}
\int_{Y} f(y) d \sigma_{*} \nu(y) & =\int_{X} f \circ \sigma(x) d \nu(x) \\
& =\int_{X} f \circ \sigma(x) \frac{d \nu}{d \mu}(x) d \mu(x) \\
& =\int_{Y} f(y) \frac{d \nu}{d \mu}\left(\sigma^{-1}(y)\right) d \sigma_{*} \mu(y)
\end{aligned}
$$

Then $\sigma_{*} \nu \ll \sigma_{*} \mu$ and

$$
\frac{d \sigma_{*} \nu}{d \sigma_{*} \mu}(y)=\frac{d \nu}{d \mu}\left(\sigma^{-1}(y)\right) \quad y \in Y .
$$

\subsection{Purely Atomic and Non-Atomic Measures}

In this section we recall that every finite Borel measure can be decomposed uniquely as a sum of two measures, one being purely atomic and the other one being continuous. As a consequence, an extremal probability measure is either purely atomic or continuous.

In order to prove Thomsen's theorem, we will show in Chapter 6 that every extremal KMS 
state corresponds to an extremal probability $m$, then $m$ has one of the properties defined below. The results in this section can be found in [12] and [25].

Definition 2.3.1. A finite Borel measure $m$ on the topological space $X$ is non-atomic or continuous when $m(\{x\})=0$ for every $x \in X$ and purely atomic if there is a Borel set $A \subset X$ such that $m(A)=m(X)$ and $m(\{a\})>0$ for all $a \in A$.

Given a measure $\mu$, we write $\mu(x)=\mu(\{x\})$.

Proposition 2.3.2. If $\mu$ is a $\sigma$-finite measure on a $\sigma$-algebra then there exist unique measures $\mu^{a}$ and $\mu^{c}$ such that $\mu=\mu^{a}+\mu^{c}$ and such that $\mu^{a}$ is purely atomic and $\mu^{c}$ is non-atomic.

\subsection{Measures on Locally Compact Spaces}

The results in this section are presented in [6]. Here we introduce the notion of Radon measures and we conclude that, if the topological space satisfies certain conditions, every probability measure is Radon.

First we prove some properties of Hausdorff spaces.

Proposition 2.4.1. Let $X$ be a Hausdorff space, and let $K$ and $L$ be disjoint compact subsets of $X$. Then there are disjoint open subsets $U$ and $V$ of $X$ such that $K \subset U$ and $L \subset V$.

Proof. We can assume that $K$ and $L$ are both non-empty (otherwise we could use $\varnothing$ as one of our open sets and $X$ as the other). Let us begin with the case where $K$ contains exactly one point, say $x$. We show that there are open disjoint sets $U_{x}, V_{x}$ such that $x \in U_{x}$ and $L \subset V_{x}$

Since $X$ is Hausdorff, for each $y \in L$ there is a pair $U_{y}, V_{y}$ of disjoint open sets such that $x \in U_{y}$ and $y \in V_{y}$. Since $L$ is compact, there is a finite family $y_{1}, \ldots, y_{n}$ such that the sets $V_{y_{1}}, \ldots, V_{y_{n}}$ cover $L$. The sets $U_{x}$ and $V_{x}$ defined by $U_{x}=\cap_{i=1}^{n} U_{y_{i}}, V_{x}=\cup_{i=1}^{n} V_{y_{i}}$ are then the required sets. 
Next consider the case where $K$ has more than one element. We have just shown that for each $x \in K$ there are disjoint open sets $U_{x}$ and $V_{x}$ such that $x \in U_{x}$ and $L \subset V_{x}$. Since $K$ is compact, there is a finite family $x_{1}, \ldots, x_{n}$ such that $U_{x_{1}}, \ldots, U_{x_{n}}$ cover $K$. The proof is complete if we define $U=\cup_{i=1}^{n} U_{x_{i}}, V=\cap_{i=1}^{n} V_{x_{i}}$.

Proposition 2.4.2. Let $X$ be a locally compact Hausdorff space, $x$ a point in $X$, and $U$ an open neighborhood of $x$. Then $x$ has an open neighborhood whose closure is compact and included in $U$.

Proof. Since $X$ is locally compact, there is an open neighborhood of $x$, say $W$, whose closure is compact. By replacing $W$ with $W \cap U$, we assume that $W$ is included in $U$. The difficulty is that $\bar{W}$ may extend outside $U$.

Use Proposition 2.4.1 to choose disjoint open sets $V_{1}$ and $V_{2}$ that separate the compact sets $\{x\}$ and $\bar{W} \backslash W$. Note that the closure of $V_{1} \cap W$ is included in $W$. In fact, suppose there exists $y \in \overline{V_{1} \cap W}$ such that $y \notin W$. Then $y \in \bar{W} \backslash W$. By definition, $V_{2}$ is a neighborhood of $y$. Since $y \in \overline{V_{1} \cap W}$, there exists $x_{1} \in V_{1} \cap W$ such that $x_{1} \in V_{2}$. This leads to a contradiction because $V_{1} \cap V_{2}=\varnothing$.

Then $\overline{V_{1} \cap W}$ is compact and included in $W$, and hence in $U$; thus $V_{1} \cap W$ is the required open neighborhood of $x$.

A subset of a topological space $X$ is a $G_{\delta}$ if it is the intersection of a sequence of open subsets of $X$, and $F_{\sigma}$ if it is the union of a sequence of closed subsets of $X$.

Proposition 2.4.3. Let $X$ be a locally compact Hausdorff space, let $K$ be a compact subset of $X$, and let $U$ be an open subset of $X$ that includes $K$. Then there is an open set $V$ of $X$ that has a compact closure and satisfies $K \subset V \subset \bar{V} \subset U$.

Proof. Proposition 2.4.2 implies that each point in $K$ has an open neighborhood whose closure is compact and included in $U$. Since $K$ is compact, some finite collection of these neighborhoods covers $K$. Let $V$ be the union of these sets in such a finite collection; then $V$ is the required set. 
Proposition 2.4.4. Let $X$ be a locally compact Hausdorff second countable space. Then each open subset of $X$ is an $F_{\sigma}$, and is in fact the union of a sequence of compact sets. Likewise, each closed subset is a $G_{\delta}$.

Proof. Suppose that $\mathcal{U}$ is a countable basis for the topology of $X$. Let $U$ be an open set in $X$. Given $x \in U$, it follows from Proposition 2.4 .2 that there exists an open neighborhood $W_{x}$ of $x$ such that $\overline{W_{x}}$ is compact and $\overline{W_{x}} \subset U$. Since $\mathcal{U}$ is the basis for the topology of $X$, there exists $V_{x} \in \mathcal{U}$ such that $x \in V_{x} \subset W_{x}$. Then $\overline{V_{x}}$ is compact and $\overline{V_{x}} \subset U$. Thus,

$$
U=\bigcup_{x \in U} \overline{V_{x}}
$$

Since each $V_{x} \in \mathcal{U}$ and $\mathcal{U}$ is countable, then $U$ is a countable union of compact sets. Therefore $U$ is $F_{\sigma}$.

Let $A \subset X$ be a closed set. Then $A^{c}$ is open, and $A^{c}$ is the union of a sequence $\left\{F_{n}\right\}$ consisting of closed sets. Hence,

$$
A=\left(A^{c}\right)^{c}=\left(\bigcup_{n=1}^{\infty} F_{n}\right)^{c}=\bigcap_{n=1}^{\infty} F_{n}^{c} .
$$

Therefore $A$ is $G_{\delta}$.

Lemma 2.4.5. Let $X$ be a locally compact Hausdorff second countable space. Given an open subset $U \subset X$, there exists a sequence $\left\{K_{n}\right\}$ of compact subsets such that $K_{n} \subset K_{n+1}$ for every $n$, and $U=\cup_{n=1}^{\infty} K_{n}$.

Proof. Let $U \subset X$ be an open set. It follows from Proposition 2.4.4 that there is a sequence of compact sets $\left\{F_{n}\right\}$ such that $U=\cup_{n} F_{n}$. Define, for each $n \geq 1, K_{n}=\cup_{i=1}^{n} F_{i}$. Clearly each $K_{n}$ is compact and $K_{n} \subset K_{n+1}$. Moreover, $U=\cup_{n=1}^{\infty} K_{n}$.

Let $X$ be a Hausdorff topological space. Then $\mathcal{B}(X)$, the Borel $\sigma$-algebra on $X$, is the $\sigma$-algebra generated by the open subsets of $X$; the Borel subsets of $X$ are those that belong to $\mathcal{B}(X)$. 
We turn to terminology for measures. Let $X$ be a Hausdorff topological space. A Borel measure on $X$ is a measure whose domain is $\mathcal{B}(X)$. Suppose that $\mathcal{A}$ is a $\sigma$-algebra on $X$ such that $\mathcal{B}(X) \subset \mathcal{A}$. A positive measure $\mu$ on $\mathcal{A}$ is Radon if

(a) each compact subset $K$ of $X$ satisfies $\mu(K)<\infty$,

(b) each set $A$ in $\mathcal{A}$ satisfies

$$
\mu(A)=\inf \{\mu(U): A \subset U \text { and } U \text { is open }\}, \text { and }
$$

(c) each open set $U$ of $X$ satisfies

$$
\mu(U)=\sup \{\mu(K): K \subset U \text { and } K \text { is compact }\}
$$

A Radon Borel measure on $X$ is a Radon measure whose domain is $\mathcal{B}(X)$. A measure that satisfies condition (b) is often called outer regular, and a measure that satisfies condition (c), inner regular.

Now we define the support of a Radon Borel measure. The following theorem is necessary to show that the support is well-defined.

Proposition 2.4.6. Let $X$ be a locally compact Hausdorff space, let $\mu$ be a Radon Borel measure on $X$. Then the union of all open subsets of $X$ that have measure zero under $\mu$ is itself an open set that has measure zero under $\mu$.

Proof. Let $\mathcal{U}$ be the collection of all open subsets of $X$ that have measure zero under $\mu$, and let $U$ be the union of the sets in $\mathcal{U}$. Then $U$ is open. If $K$ is a compact subset of $U$, then $K$ can be covered by a finite collection $U_{1}, \ldots, U_{n}$ of sets that belong to $\mathcal{U}$, and so we have

$$
\mu(K) \leq \sum_{i=1}^{n} \mu\left(U_{i}\right)=0 .
$$

Since $K$ is arbitrary, it follows from the definition of Radon measure that $\mu(U)=0$. 
It follows from Proposition 2.4.6 that, for $X, \mu$, there is the largest open subset $U \subset X$ with $\mu(U)=0$. Then we define the support as follows.

Definition 2.4.7. Let $X$ be a locally compact Hausdorff space, and $\mu$ a Radon Borel measure on $X$. We define the support of $\mu$ as the complement of the largest open subset of $X$ with measure zero. We denote the support of $X$ by $\operatorname{supp}(\mu)$.

Note that $\operatorname{supp}(\mu)$ is closed. Now we prove some properties of Radon measures.

Lemma 2.4.8. Let $X$ be a Hausdorff space in which each open set is an $F_{\sigma}$, and let $\mu$ be a finite Borel measure on $X$. Then each Borel subset $A$ of $X$ satisfies

$$
\begin{aligned}
& \mu(A)=\inf \{\mu(U): A \subset U \text { and } U \text { is open }\} \\
& \mu(A)=\sup \{\mu(F): F \subset A \text { and } F \text { is closed }\}
\end{aligned}
$$

In particular, $\mu$ is Radon.

Proof. Let $\mathcal{R}$ denote the set of Borel sets $A \subset X$ that satisfy conditions (2.4) and (2.5). We prove that $\mathcal{R}$ contains all open subsets of $X$. Let $U \subset X$ open. Clearly $U$ satisfies 2.4. By hypothesis there exists a sequence $\left\{F_{n}\right\}$ of closed sets such that $U=\cup_{n} F_{n}$. We can assume that $F_{n} \subset F_{n+1}$ for each $n$ without loss of generality. Then $\mu(U)=\lim _{n} \mu\left(F_{n}\right)$. Therefore (2.5) holds.

Now we show that conditions (2.4) and 2.5 hold for an arbitrary Borel set $A$ if, and only if, for every $\varepsilon>0$ there are $U$ open, $F$ closed, such that

$$
F \subset A \subset U \text { and } \mu(U \backslash F)<\varepsilon
$$

In fact, assume (2.4) and 2.5 hold. Let $A$ be measurable. Given $\varepsilon>0$, by (2.4) there exists $U$ open such that $A \subset U$ and

$$
\mu(U)<\mu(A)+\varepsilon / 2 .
$$


Aplying (2.5), there exists $F \subset A$ closed satisfying

$$
\mu(F)>\mu(A)-\varepsilon / 2 .
$$

Then, by (2.7) and (2.8), we have

$$
\mu(U \backslash F)=\mu(U)-\mu(F)<\left(\mu(A)+\frac{\varepsilon}{2}\right)-\left(\mu(A)-\frac{\varepsilon}{2}\right)=\varepsilon .
$$

Then (2.6) holds.

Conversely, assume (2.6) holds. Given $\varepsilon>0$, there are $U$ open, $F$ closed, such that $F \subset A \subset U$ and $\mu(U \backslash F)<\varepsilon$. Hence,

$$
\begin{aligned}
\mu(A) \leq \mu(U) & =\mu(A)+\mu(U \backslash A) \\
& \leq \mu(A)+\mu(U \backslash F), \text { since } F \subset A, \\
& <\mu(A)+\varepsilon,
\end{aligned}
$$

and

$$
\begin{aligned}
\mu(F) \leq \mu(A) & =\mu(F)+\mu(A \backslash F) \\
& \leq \mu(F)+\mu(U \backslash F), \text { since } A \subset U, \\
& <\mu(F)+\varepsilon .
\end{aligned}
$$

Since $\varepsilon>0$ is arbitrary, it follows that conditions $(2.4)$ and $(2.5)$ are satisfied.

We can now show that $\mathcal{R}$ is a $\sigma$-algebra. Clearly $\varnothing \in \mathcal{R}$, since $\varnothing$ is open. Given $A \in \mathcal{R}$, $\varepsilon>0$, there are $U$ open, $F$ closed such that $F \subset A \subset U$ and $\mu(U \backslash F)<\varepsilon$. Then $F^{c}$ is open, $U^{c}$ is closed, and $U^{c} \subset A^{c} \subset F^{c}$. Since $F^{c} \backslash U^{c}=U \backslash F$, it follows that $\mu\left(F^{c} \backslash U^{c}\right)=\mu(U \backslash F)<\varepsilon$. Therefore $A^{c} \in \mathcal{R}$.

Let $\left\{A_{n}\right\}$ be a sequence of sets in $\mathcal{R}$. Then, for every $n \geq 1$, there exists $U_{n}$ open, $F_{n}$ 
closed such that

$$
F_{n} \subset A_{n} \subset U_{n} \text { and } \mu\left(U_{n} \backslash F_{n}\right)<\frac{\varepsilon}{2^{n+1}}
$$

Let $U=\cup_{n} U_{n}$ and $F=\cup_{n} F_{n}$. Then $U, F$ satisfy the relations $F \subset \cup_{n} A_{n} \subset U$ and

$$
\mu(U \backslash F) \leq \mu\left(\bigcup_{n=1}^{\infty}\left(U_{n} \backslash F_{n}\right)\right) \leq \sum_{n=1}^{\infty} \mu\left(U_{n} \backslash F_{n}\right)<\sum_{n=1}^{\infty} \frac{\varepsilon}{2^{n+1}}=\frac{\varepsilon}{2}
$$

The set $U$ is open, but the set $F$ can fail to be closed. However for each $N$ the set $\cup_{n=1}^{N} F_{n}$ is closed, and since

$$
\mu(U \backslash F)=\mu(U)-\mu(F)=\mu(U)-\lim _{N \rightarrow \infty} \mu\left(\bigcup_{n=1}^{N} F_{n}\right)
$$

we can choose $N$ such that

$$
\mu\left(U \backslash \bigcup_{n=1}^{N} F_{n}\right)<\varepsilon
$$

Thus $U$ and $\cup_{n=1}^{N} F_{k}$ are the sets required in (2.6), and $\mathcal{R}$ is closed under countable unions.

We have now shown that $\mathcal{R}$ is a $\sigma$-algebra on $X$ that contains the open sets. Since $\mathcal{B}(X)$ is the smallest $\sigma$-algebra on $X$ that contains the open sets, it follows that $\mathcal{B}(X) \subset \mathcal{R}$. Therefore this lemma is proved.

Remark 2.4.9. Let $X$ be a locally compact Hausdorff second countable space, then every probability measure on $X$ is Radon. In fact, it follows from Proposition 2.4.4 that every open set is $G_{\delta}$. Then, given a probability measure $\mu$ on $X$, it follows from Lemma 2.4.8 that $\mu$ is Radon.

We assume in Lemma 2.4.8 that the measure $\mu$ is finite, but this result can be generalized to $\sigma$-finite measures that are finite on compact sets. 
Proposition 2.4.10. Let $X$ be a locally compact Hausdorff space that has a countable basis, and let $\mu$ be a Borel measure on $X$ that is finite on compact sets. Then $\mu$ is Radon.

Proof. First consider the inner regularity of $\mu$. Let $U$ be an open subset of $X$. Lemma 2.4.5 implies that $U$ is the union of a sequence $\left\{K_{j}\right\}$ of compact subsets, then

$$
\mu(U)=\lim _{n \rightarrow \infty} \mu\left(\bigcup_{j=1}^{n} K_{j}\right) .
$$

The inner regularity follows.

Let $\left\{U_{n}\right\}$ be a sequence of open sets such that $X=\cup_{n} U_{n}$ and such that $\mu\left(U_{n}\right)<\infty$ holds for each $n$ (for instance, take a countable basis $\mathcal{U}$ for $X$, and arrange in a sequence those sets $U$ in $\mathcal{U}$ for which $\bar{U}$ is compact).

For each $n$ define a Borel measure $\mu_{n}$ on $X$ by $\mu_{n}(A)=\mu\left(A \cap U_{n}\right)$. The measures $\mu_{n}$ are finite, and so Lemma 2.4 .8 implies that they are outer regular. Hence if $A$ belongs to $\mathcal{B}(X)$ and if $\varepsilon$ is a positive number, then for each $n$ there is an open set $V_{n}$ that includes $A$ and satisfies $\mu_{n}\left(V_{n}\right)<\mu_{n}(A)+\varepsilon / 2^{n}$. Consequently,

$$
\mu\left(\left(U_{n} \cap V_{n}\right) \backslash A\right)<\varepsilon / 2^{n}
$$

Then set $V$ defined by $V=\cup_{n}\left(U_{n} \cap V_{n}\right)$ is open, includes $A$ and satisfies

$$
\mu(V \backslash A) \leq \sum_{n} \mu\left(\left(U_{n} \cap V_{n}\right) \backslash A\right)<\varepsilon
$$

Hence $\mu(V) \leq \mu(A)+\varepsilon$, and the outer regularity of $\mu$ follows.

Assume $X$ is a locally compact second countable Hausdorff space. By definition, a Radon measure $\mu$ on $X$ is finite on compact subsets of $X$. It follows from Proposition 2.4 .10 that a Borel measure on $X$ is Radon if, and only if, it is finite on the compact subsets of $X$.

Proposition 2.4.11. [6, Proposition 7.2.6] Let $X$ be a Hausdorff space, let $\mathcal{A}$ be a $\sigma$-algebra 
on $X$ that includes $\mathcal{B}(X)$, and let $\mu$ be a Radon measure on $\mathcal{A}$. If $A$ belongs to $\mathcal{A}$ and is $\sigma$-finite under $\mu$, then

$$
\mu(A)=\sup \{\mu(K): K \subset A \text { and } K \text { is compact }\} .
$$

Remark 2.4.12. Let $X$ be a locally compact second countable Hausdorff space, and $\mu$ a Borel measure which is finite on compact subsets of $X$. It follows from Propositions 2.4.10 and 2.4.11 that, for every $A \subset X$ Borel, we have

$$
\begin{aligned}
& \mu(A)=\inf \{\mu(U): A \subset U \text { and } U \text { is open }\} \\
& \mu(A)=\sup \{\mu(K): K \subset A \text { and } K \text { is compact }\} .
\end{aligned}
$$

Lemma 2.4.13. Let $X$ be a locally compact Hausdorff second countable space, $\mu$ be a Radon Borel measure on $X, B \subset X$ a Borel set, $U \subset X$ an open set satisfying $B \subset U$. Given a continuous non-negative function $f$ on $X$, we have

$$
\int_{B} f(x) d \mu(x)=\inf _{\substack{B \subset V \subset U \\ V \text { open }}} \int_{V} f(x) d \mu(x)
$$

Proof. Since $f$ is continuous and non-negative, we can define the Borel measure $\nu$ on $X$ by

$$
\nu(A)=\int_{A} f(x) d \mu(x), \quad A \text { Borel set. }
$$

The function $f$ is continuous and $\mu$ is finite on compact subsets, then $\nu$ is finite on compact subsets of $X$. Hence, from Proposition 2.4.10, $\nu$ is Radon. Therefore, for every $B \subset X$ Borel,

$$
\int_{B} f(x) d \mu(x)=\nu(B)=\inf _{\substack{B \subset V \\ V \text { open }}} \nu(V)=\inf _{\substack{B \subset V \\ V \text { open }}} \int_{V} f(x) d \mu(x) .
$$

For every open set $V$ such that $B \subset V$, it follows that $\mu(B) \leq \mu(V \cap U) \leq \mu(U)$ and 
$V \cap U$ is open. Hence, we can take the infimum over the open sets $V$ such that $B \subset V \subset U$. Therefore,

$$
\int_{B} f(x) d \mu(x)=\nu(B)=\inf _{\substack{B \subset V \subset U \\ V \text { open }}} \nu(V)=\inf _{\substack{B \subset V \subset U \\ V \text { open }}} \int_{V} f(x) d \mu(x)
$$

Lemma 2.4.14. Let $X$ be a locally compact Hausdorff second countable space and $\mu$ a Radon measure on $X$. Given an open subset $U \subset X$, we have

$$
\mu(U)=\sup _{\substack{f \in C_{c}(X) \\ 0 \leq f \leq \chi_{U}}} \int_{X} f(x) d \mu(x) .
$$

Proof. - Assume $\mu(U)<\infty$.

Note that for every $f \in C_{c}(X)$ such that $0 \leq f \leq \chi_{U}$, we have

$$
\int_{X} f(x) d \mu(x) \leq \mu(U)
$$

Given $\varepsilon>0$, there is a compact set $K \subset U$ satisfying $\mu(U \backslash K)<\varepsilon$ by Remark 2.4.12. Since $X$ is locally compact Hausdorff, there is an open set $V$ such that $\bar{V}$ is compact and $K \subset V \subset \bar{V} \subset U$ by Proposition 2.4.3.

By Urysohn's lemma, there exists a continuous function $f$ assuming values in the interval $[0,1]$ such that $f$ equals one on $K$ and vanishes outside $\bar{V} \subset U$. Then $f \in$ $C_{c}(X)$ and $0 \leq f \leq \chi_{U}$.

Using the fact that $\mu(U)-\mu(K) \leq \varepsilon$, we have $\mu(K) \geq \mu(U)-\varepsilon$. Hence,

$$
\begin{aligned}
\int_{X} f(x) d \mu(x) & =\mu(K)+\int_{U \backslash K} f(x) d \mu(x) \\
& \geq \mu(K) \\
& \geq \mu(U)-\varepsilon .
\end{aligned}
$$


Since $\varepsilon$ is arbitrary, we have

$$
\mu(U)=\sup _{\substack{f \in C_{c}(X) \\ 0 \leq f \leq \chi_{U}}} \int_{X} f(x) d \mu(x) .
$$

- Suppose $\mu(U)=\infty$.

Let $n$ be a natural number. By Remark 2.4.12, there exists a compact set $K_{n} \subset U$ such that $\mu\left(K_{n}\right) \geq n$. From Urysohn's lemma, we can choose a continuous compactly supported function $f_{n}$ assuming values in the interval $[0,1]$ such that $f_{n}(x)=1$ for every $x \in K_{n}$ and $f_{n}$ vanishes of $U$. Hence,

$$
\int_{X} f_{n}(x) d \mu(x) \geq \int_{K_{n}} f_{n}(x) d \mu(x)=\mu\left(K_{n}\right) \geq n .
$$

Therefore,

$$
\sup _{\substack{f \in C_{c}(X) \\ 0 \leq f \leq \chi_{U}}} \int_{X} f(x) d \mu(x) \geq \sup _{n \in \mathbb{N}} \int_{X} f_{n}(x) d \mu(x)=\infty .
$$

Hence the result follows.

\section{$2.5 \quad \mu$-Measurable Functions}

In this section we define the $\mu$-completion of a $\sigma$-algebra. The definition here can be found in [6]. This $\sigma$-algebra will be necessary to understand one of the conditions in Neshveyev's first theorem.

Definition 2.5.1. Let $(X, \mathcal{A})$ be a measurable space and let $\mu$ be a measure on $\mathcal{A}$. The completion of $\mathcal{A}$ under $\mu$ is the collection $\mathcal{A}_{\mu}$ of subsets $A$ of $X$ for which there are sets $E$ and $F$ in $\mathcal{A}$ such that 


$$
E \subset A \subset F \text { and } \mu(F \backslash E)=0 \text {. }
$$

A set that belongs to $\mathcal{A}_{\mu}$ is said to be $\mu$-measurable.

In fact, $\mathcal{A}_{\mu}$ is a $\sigma$-algebra on $X$. We say that a function $f$ on $X$ is $\mu$-measurable if it is measurable with respect to the $\sigma$-algebra $\mathcal{A}_{\mu}$. Note that if $f$ is measurable with respect to the $\sigma$-algebra $\mathcal{A}$, then $f$ is $\mu$-measurable.

Lemma 2.5.2. Let $X$ be a topological space and $\mu$ a Borel purely atomic probability measure on $X$. Every complex-valued function on $X$ is $\mu$-measurable.

Proof. Let $f$ be a complex valued function on $X$. Let $I$ be the set of points $x \in X$ such that $\mu(\{x\})>0$, then $I$ is countable $\mu(X \backslash I)=0$. Given $V \subset \mathbb{C}$ measurable, let $A=f^{-1}(V)$ and $J=I \backslash A$. Then $I, J$ are measurable since both are countable.

Note that $I \cap A \subset A \subset X \backslash J$. Since $(X \backslash J) \backslash I \subset X \backslash I$, it follows that

$$
\mu((X \backslash J) \backslash I) \leq \mu(X \backslash I)=0
$$

Then $A$ is $\mu$-measurable and, therefore, $f$ is $\mu$-measurable.

\subsection{Vector-Valued Integration}

Now we introduce the concept of vector-valued integral, that is, the integral of functions $f: \mathbb{R} \rightarrow B$ where $B$ is a complex Banach space. This section is based on [21]. We will need this notion to prove Proposition 5.1.19 on page 105, and then define KMS states on a arbitrary $\mathrm{C}^{*}$-algebra.

Recall that one of the main steps in the construction of the Lebesgue integral is the notion

of simple functions. A function $\varphi: \mathbb{R} \rightarrow \mathbb{R}$ is simple if there are $A_{1}, \cdots, A_{n}$ measurable sets 
and $a_{1}, \ldots, a_{n}$ real numbers such that

$$
\varphi=\sum_{i=1}^{n} a_{i} \chi_{A_{i}} .
$$

Then we define its integral by

$$
\int_{\mathbb{R}} \varphi(t) d \mu(t)=\sum_{i=1}^{n} a_{i} \mu\left(A_{i}\right) .
$$

Then, under certain conditions, the integral of a measurable function can be approximated by the integral of simple functions. We will try to define the integral of vector-valued functions similarly.

Definition 2.6.1. Let $\mu$ be a Borel measure on $\mathbb{R}$ and $B$ a Banach space. A function $\varphi: \mathbb{R} \rightarrow B$ is simple if there are $a_{1}, \ldots, a_{n} \in B$, and $A_{1}, \ldots, A_{n}$ Borel subsets of $B$ such that

$$
\varphi=\sum_{i=1}^{n} a_{i} \chi_{A_{i}}
$$

and each $\chi_{A_{i}}: \mathbb{R} \rightarrow \mathbb{R}$ is defined by

$$
\chi_{A_{i}}(x)= \begin{cases}1, & \text { if } x \in A_{i}, \\ 0, & \text { if } x \notin A_{i},\end{cases}
$$

for $x \in \mathbb{R}$. We call 2.11) a representation of $\varphi$.

In the next example, we show that the representation (2.11) is not necessarily unique.

Example 2.6.2. Let $\varphi: \mathbb{R} \rightarrow \mathbb{R}^{3}$ be defined by

$$
\varphi(x)= \begin{cases}(1,1,1), & \text { if } 0<x \leq 1, \\ (1,2,2), & \text { if } 1<x \leq 2, \\ (1,2,3), & \text { if } 2<x \leq 3 .\end{cases}
$$


Then,

$$
\begin{aligned}
\varphi & =(1,1,1) \chi_{(0,1]}+(1,2,2) \chi_{(1,2]}+(1,2,3) \chi_{(2,3]} \\
& =(1,1,1) \chi_{(0,3]}+(0,1,1) \chi_{(1,3]}+(0,0,1) \chi_{(2,3]}
\end{aligned}
$$

Therefore $\varphi$ is simple and can be written in at least two different representations.

Definition 2.6.3. Let $\mu$ be a Borel measure on $\mathbb{R}$ and $B$ a Banach space. Given a simple function $\varphi$ with representation 2.11 , we define its integral by

$$
\int_{\mathbb{R}} \varphi(x) d \mu(x)=\sum_{i=1}^{n} a_{i} \mu\left(A_{i}\right) .
$$

Lemma 2.6.4. The integral in Definition 2.6 .3 is well-defined, that is, it does not depend on the representation.

Proof. Let $B$ be a Banach space. Given a simple function $\varphi: \mathbb{R} \rightarrow B$, let $a_{1}, \ldots, a_{n} \in B$, $b_{1}, \ldots, b_{m} \in B$, and let $A_{1}, \ldots, A_{n}, B_{1}, \ldots, B_{m}$ be Borel sets such that

$$
\varphi=\sum_{i=1}^{n} a_{i} \chi_{A_{i}}=\sum_{j=1}^{m} b_{i} \chi_{A_{i}}
$$

Let

$$
x=\sum_{i=1}^{n} a_{i} \mu\left(A_{i}\right) \quad \text { and } \quad y=\sum_{j=1}^{m} b_{j} \mu\left(B_{j}\right) .
$$

Note that $x, y \in B$. Choose an arbitrary $\Lambda \in B^{*}$. Then $\Lambda \circ \varphi: \mathbb{R} \rightarrow \mathbb{C}$ is a simple function with

$$
\Lambda \circ \varphi=\sum_{i=1}^{n} \Lambda\left(a_{i}\right) \chi_{A_{i}}=\sum_{j=1}^{m} \Lambda\left(b_{i}\right) \chi_{A_{i}} .
$$

Since the integral of complex-valued functions does not depend on the representation, we 
have

$$
\begin{aligned}
\int_{\mathbb{R}}(\Lambda \circ \varphi)(t) d \mu(t)=\sum_{i=1}^{n} \Lambda\left(a_{i}\right) \mu A_{i} & =\sum_{j=1}^{m} \Lambda\left(b_{i}\right) \mu B_{i} \\
=\Lambda\left(\sum_{i=1}^{n} a_{i} \mu A_{i}\right) & =\Lambda\left(\sum_{j=1}^{m} b_{j} \mu B_{j}\right) \\
=\Lambda(x) & =\Lambda(y)
\end{aligned}
$$

Since $\Lambda$ is arbitrary and $B^{*}$ separates points in $B$, it follows that $x=y$.

Remark 2.6.5. Given a simple function $\varphi: \mathbb{R} \rightarrow B, \Lambda \in B^{*}, \Lambda \circ \varphi: \mathbb{R} \rightarrow \mathbb{C}$ is a simple function. Note that we used the property

$$
\Lambda\left(\int_{\mathbb{R}} \varphi(t) d \mu(t)\right)=\int_{\mathbb{R}} \Lambda(\varphi(t)) d \mu(t)
$$

in Lemma 2.6.4 to show that the integral is well-defined. Similarly, we will define the integral in such a way that this property holds when we replace $\varphi$ by a Borel function $f: \mathbb{R} \rightarrow B$. Given $\Lambda \in B^{*}$, we denote $\Lambda f=\Lambda \circ f$. Note that both $\Lambda f$ and $\Lambda \varphi$ are measurable functions. Definition 2.6.6. Given a Banach space $B$, a function $f: \mathbb{R} \rightarrow B$ is weakly measurable if $\Lambda f$ is measurable for every $\Lambda \in X^{*}$.

Remark 2.6.7. Note that every Borel function $f: \mathbb{R} \rightarrow B$ is weakly measurable. In particular, every continuous function from $\mathbb{R}$ to $B$ is weakly measurable.

Definition 2.6.8. Let $\mu$ be a Borel measure on $\mathbb{R}$. Given a Banach space $B$, let $f: \mathbb{R} \rightarrow B$ be weakly measurable. If there exists $y \in B$ such that for every $\Lambda \in B^{*}$,

$$
\Lambda y=\int_{\mathbb{R}} \Lambda f(t) d \mu(t),
$$

then we define the integral of $f$ by

$$
\int_{\mathbb{R}} f(t) d \mu(t)=y .
$$


Remark 2.6.9. Note that there exists at most one $y$ such that 2.12 holds. This follows from the fact that $B^{*}$ separates points in $B$.

Definition 2.6.10. Given a Banach space $B, C_{c}(\mathbb{R}, B)$ denotes the space of compactly supported functions $f: \mathbb{R} \rightarrow B$ which are continuous. Recall that the support of $f$ is the closure of the set $\{t \in \mathbb{R}: f(t) \neq 0\}$.

Note that every function $f \in C_{c}(\mathbb{R}, B)$ is weakly measurable.

Given a Banach space $B$, we define the norm on $C_{c}(\mathbb{R}, B)$ by

$$
\|f\|_{\infty}=\sup _{t \in \mathbb{R}}\|f(t)\|
$$

Lemma 2.6.11. Let $\mu$ be a Borel measure on $\mathbb{R}, B$ a Banach space and $f \in C_{c}(\mathbb{R}, B)$ such that there exists $y=\int_{\mathbb{R}} f(t) d \mu(t)$. Then

$$
\|y\| \leq \int_{\mathbb{R}}\|f(t)\| d \mu(t)
$$

Proof. Since $B$ is a Banach space, we have

$$
\|y\|=\sup _{\substack{\Lambda \in B^{*} \\\|\Lambda\| \leq 1}}|\Lambda y|
$$

However, for every $\Lambda \in B^{*}$ such that $\|\Lambda\| \leq 1$, we have

$$
|\Lambda y|=\left|\int_{\mathbb{R}} \Lambda f(t) d \mu(t)\right| \leq \int_{\mathbb{R}}|\Lambda f(t)| d \mu(t) \leq \int_{\mathbb{R}}\|\Lambda\|\|f(t)\| d \mu(t) \leq \int_{\mathbb{R}}\|f(t)\| d \mu(t) .
$$

Lemma 2.6.12. Let $\mu$ be a Radon measure on $\mathbb{R}$. Let $f \in C_{c}(\mathbb{R}, B)$. Then, for every $\varepsilon>0$, there are $A_{1}, \ldots A_{n}$ disjoint Borel sets, $t_{1}, \ldots, t_{n} \in \mathbb{R}$, such that the function

$$
\varphi=\sum_{i=1}^{n} f\left(t_{i}\right) \chi_{A_{i}}
$$


satisfies the following property: $\|\varphi-f\|_{\infty} \leq \varepsilon$.

Proof. Let $\varepsilon>0$. Since $f$ is continuous, for every $t \in \mathbb{R}$, there exists an open set $U_{t}$ such that for every $s \in U_{t},\|f(s)-f(t)\|<\varepsilon$.

Let $K$ be the support of $f$. Then there are $t_{1}, \ldots t_{n} \in K$ such that $U_{t_{1}}, \ldots, U_{t_{n}}$ is an open cover for $K$. Let, for $i=1, \ldots, n$,

$$
A_{i}= \begin{cases}U_{t_{1}} & \text { if } i=1 \\ U_{t_{i}} \backslash A_{i-1} & \text { if } i=2, \ldots, n .\end{cases}
$$

Then each $A_{i}$ is Borel, and $\cup_{i=1}^{n} A_{i}=\cup_{i=1}^{n} U_{t_{i}}$. Define $\varphi$ by

$$
\varphi=\sum_{i=1}^{n} f\left(t_{i}\right) \chi_{A_{i}} .
$$

Now we prove that $\|\varphi-f\|_{\infty} \leq \varepsilon$. Let $t \in \mathbb{R}$. If $t \notin \cup_{i=1}^{n} U_{t_{i}}$, then $\varphi(t)=0$ by definition. Moreover, $t \notin K$, since $U_{t_{1}}, \ldots, U_{t_{n}}$ cover $K$. Then $f(t)=0$ and $\|f(t)-\varphi(t)\|=0 \leq \varepsilon$.

Assume $t \in \cup_{i=1}^{n} U_{t_{i}}$. By definition of $A_{1}, \ldots, A_{n}$, there exists a unique $i$ such that $t \in A_{i}$. Hence $\varphi(t)=f\left(t_{i}\right)$. Since $A_{i} \subset U_{t_{i}}$, we have

$$
\|\varphi(t)-f(t)\|=\left\|f\left(t_{i}\right)-f(t)\right\|<\varepsilon .
$$

Therefore,

$$
\|\varphi-f\|_{\infty}=\sup _{t \in \mathbb{R}}\|\varphi(t)-f(t)\| \leq \varepsilon .
$$

In order to prove the existence of the integral of functions in $C_{c}(\mathbb{R}, B)$, we will state Theorem 2.6.14, which is an application of a theorem proved in [22].

Definition 2.6.13. Let $X$ be a normed vector space. Given a subset $S$ of $X$, we define 
its convex hull as the smallest convex set containing $S$. We denote the convex hull of $S$ by $\operatorname{co}(S)$

Theorem 2.6.14. [22, Theorem 3.25] Suppose $H$ is the convex hull of a compact set $K$ in a Banach space $B$. Then $\bar{H}$ is compact.

Theorem 2.6.15. Let $\mu$ be a Radon measure on $\mathbb{R}$. Let $B$ be a Banach space. Given $f \in C_{c}(\mathbb{R}, B)$, the integral of $f:$

$$
y=\int_{\mathbb{R}} f(t) d \mu(t)
$$

exists.

Proof. Assume $\mu$ is a probability measure.

Let $K=\operatorname{supp}(f)$. Let $L=f(K) \cup\{0\}$. This set is compact because $f$ is continuous and $K$ is compact. Define $H$ to be the closure of $\operatorname{co}(L)$. Then $H$ is compact by Theorem 2.6.14.

Given $k \in \mathbb{N}$ with $k \geq 1$, it follows from Lemma 2.6.12 that there is a simple function $\varphi^{(k)}: \mathbb{R} \rightarrow B$ such that there are disjoint Borel sets $A_{1}^{(k)}, \ldots, A_{n_{k}}^{(k)}$, and $t_{1}^{(k)}, \ldots, t_{n_{k}}^{(k)} \in \mathbb{R}$ satisfying

$$
\varphi^{(k)}=\sum_{i=1}^{n_{k}} f\left(t_{i}^{(k)}\right) \chi_{A_{i}^{(k)}} \quad \text { and } \quad\left\|\varphi^{(k)}-f\right\|_{\infty}<\frac{1}{k}
$$

Let

$$
y_{k}=\int_{\mathbb{R}} \varphi^{(k)} d \mu(t)=\sum_{i=1}^{n_{k}} f\left(t_{i}^{(k)}\right) \mu\left(A_{i}^{(k)}\right) .
$$

Since the sets $A_{1}^{(k)}, \ldots, A_{n_{k}}^{(k)}$ are disjoint and $\mu$ is a probability measure, we have

$$
\sum_{i=1}^{n_{k}} \mu\left(A_{i}^{(k)}\right) \leq 1 \quad \text { and } \quad\left\|y_{k}\right\| \leq\|f\|_{\infty} .
$$


Moreover, since each $f\left(t_{i}^{(k)}\right) \in H$ and $0 \in H$, it follows from 2.13) and (2.14) that $y_{k} \in H$. $H$ is compact, then $\left\{y_{k}\right\}_{k \in \mathbb{N}}$ has a subsequence $\left\{y_{k_{j}}\right\}_{j \in \mathbb{N}}$ converging to some $y \in H$.

Let $\Lambda \in B^{*}$. Assume $\Lambda \neq 0$ without loss of generality. Since $\Lambda$ is continuous, we have $\Lambda y_{k_{j}} \rightarrow \Lambda y$. However, by Remark 2.6.5.

$$
\Lambda y_{k_{j}}=\Lambda\left(\int_{\mathbb{R}} \varphi^{\left(k_{j}\right)}(t) d \mu(t)\right)=\int_{\mathbb{R}} \Lambda \varphi^{\left(k_{j}\right)}(t) d \mu(t)
$$

By definition, each $\varphi^{\left(k_{j}\right)}$ satisfies $\left\|\varphi^{\left(k_{j}\right)}(t)\right\| \leq\|f\|_{\infty}$. Hence, for $t \in \mathbb{R}$,

$$
\left|\Lambda \varphi^{\left(k_{j}\right)}(t)\right| \leq\|\Lambda\|\left\|\varphi^{\left(k_{j}\right)}(t)\right\| \leq\|\Lambda\|\|f\|_{\infty} .
$$

Moreover, $\Lambda \varphi^{\left(k_{j}\right)}$ converges to $\Lambda f$ pointwise. Since $\mu$ is a probability measure, we can apply the dominated convergence theorem, obtaining

$$
\int_{\mathbb{R}} \Lambda f(t) d \mu(t)=\lim _{j \rightarrow \infty} \int_{\mathbb{R}} \Lambda \varphi^{\left(k_{j}\right)}(t) d \mu(t)=\lim _{j \rightarrow \infty} \Lambda y_{k_{j}}=\Lambda y
$$

Since $\Lambda$ is arbitrary, we have

$$
y=\int_{\mathbb{R}} f(t) d \mu(t)
$$

Now assume $\mu$ is an arbitrary Radon measure on $\mathbb{R}$. Let $K$ be the support of $f$.

Suppose $\mu(K)=0$, then for each $\Lambda \in B^{*}$,

$$
\int_{\mathbb{R}} \Lambda(f(t)) d \mu(t)=\int_{K} \Lambda(f(t)) d \mu(t)=0 .
$$

Therefore $\int_{\mathbb{R}} f(t) d \mu(t)=0$.

Now suppose $\mu(K)>0$. Define the measure $\widetilde{\mu}$ on $\mathbb{R}$ by

$$
\widetilde{\mu}(I)=\frac{\mu(I \cap K)}{\mu(K)}
$$


for every Borel set $I \subset \mathbb{R}$. Then $\widetilde{\mu}$ is a probability Borel measure on $\mathbb{R}$.

Let $y=\int_{\mathbb{R}} f(t) d \widetilde{\mu}(t)$. Then, for every $\Lambda \in B^{*}$,

$$
\begin{aligned}
\Lambda y & =\int_{\mathbb{R}} \Lambda(f(t)) d \widetilde{\mu}(t) \\
& =\int_{K} \Lambda(f(t)) d \widetilde{\mu}(t) \\
& =\frac{1}{\mu(K)} \int_{K} \Lambda(f(t)) d \mu(t) \\
& =\frac{1}{\mu(K)} \int_{\mathbb{R}} \Lambda(f(t)) d \mu(t) .
\end{aligned}
$$

Therefore,

$$
\mu(K) y=\int_{\mathbb{R}} f(t) d \mu(t)
$$

Proposition 2.6.16. Let $\mu$ be a Borel measure on $\mathbb{R}$ and let $B$ be a Banach space. Let $f: \mathbb{R} \rightarrow B$ be a continuous function such that $\int_{-\infty}^{\infty}\|f(t)\| d \mu(t)<\infty$. Then the integral $\int_{\mathbb{R}} f(t) d \mu(t)$ exists.

Proof. Let, for each $n, h_{n}$ be a continuous function such that $h_{n}$ equals 1 in the closed interval $[-n, n]$ and vanishes outside $]-n-1, n+1\left[\right.$. For each $n, f h_{n} \in C_{c}(\mathbb{R}, B)$. Define, for every $n$,

$$
y_{n}=\int_{\mathbb{R}} h_{n}(t) f(t) d \mu(t) .
$$

In order to show that the sequence of $y_{n}$ converges, we only need to prove that $\left\{y_{n}\right\}$ is a Cauchy sequence because $B$ is complete. Given $\varepsilon>0$, let $n_{0}$ be such that

$$
\int_{|t| \geq n_{0}}\|f(t)\|<\frac{\varepsilon}{2}
$$


Given $n, m \geq n_{0}$, assume $n \geq m$ without loss of generality. By definition, we have

$$
h_{n}(t)=h_{m}(t)=1 \quad \text { for } t \text { satisfying }|t|<n_{0} .
$$

In this case, $\left|h_{n}(t)-h_{m}(t)\right|=0$. Then,

$$
\begin{aligned}
\left\|y_{n}-y_{m}\right\| & =\left\|\int_{\mathbb{R}} h_{n}(t) f(t) d \mu(t)-\int_{\mathbb{R}} h_{m}(t) f(t) d \mu(t)\right\| \\
& =\left\|\int_{\mathbb{R}}\left(h_{n}(t)-h_{m}(t)\right) f(t) d \mu(t)\right\| \\
& \leq \int_{\mathbb{R}}\left|h_{n}(t)-h_{m}(t)\right|\|f(t)\| d \mu(t) \\
& =\int_{|t| \geq n_{0}}\left|h_{n}(t)-h_{m}(t)\right|\|f(t)\| d \mu(t), \quad \text { by }[2.16), \\
& \leq \int_{|t| \geq n_{0}}\left(\left|h_{n}(t)\right|+\left|h_{m}(t)\right|\right)\|f(t)\| d \mu(t) \\
& \leq 2 \int_{|t| \geq n_{0}}\|f(t)\| d \mu(t), \quad \text { because } h_{n}, h_{m} \text { assume values in }[0,1], \\
& \leq 2 \frac{\varepsilon}{2}=\varepsilon .
\end{aligned}
$$

Therefore $y_{n} \rightarrow y$ for some $y \in B$.

Now we prove that $\int_{\mathbb{R}} \Lambda\left(h_{n}(t) f(t)\right) d \mu(t) \rightarrow \int_{\mathbb{R}} \Lambda(f(t)) d \mu(t)$. For every $t$, we have

$$
\left|\Lambda\left(h_{n}(t) f(t)\right)\right| \leq\|\lambda\|\|f(t)\| .
$$

By assumption, the function $t \mapsto\|f(t)\|$ is integrable. Then, the dominated convergence theorem implies,

$$
\lim _{n \rightarrow \infty} \int_{\mathbb{R}} \Lambda\left(h_{n}(t) f(t)\right) d \mu(t)=\int_{\mathbb{R}}\left(\lim _{n \rightarrow \infty} \Lambda\left(h_{n}(t) f(t)\right)\right) d \mu(t)=\int_{\mathbb{R}} \Lambda(f(t)) d \mu(t) .
$$


Note that, for every $n$,

$$
\Lambda\left(\int_{\mathbb{R}} h_{n}(t) f(t) d \mu(t)\right)=\int_{\mathbb{R}} \Lambda\left(h_{n}(t) f(t)\right) d \mu(t) .
$$

The left-hand side equals to $\Lambda y_{n}$ and thus converges to $\Lambda y$. As we already proved, the right-hand side converges to $\int_{\mathbb{R}} \Lambda(f(t)) d \mu(t)$. Therefore,

$$
\Lambda y=\int_{\mathbb{R}} \lambda(f(t)) d \mu(t)
$$

$\Lambda$ is arbitrary, then the integral

$$
y=\int_{\mathbb{R}} f(t) d \mu(t)
$$

exists.

Corollary 2.6.17. Let $B$ be a Banach space and $\mu$ a Borel measure on $X$. Let $f: \mathbb{R} \rightarrow B$ be a continuous function such that $t \mapsto\|f(t)\|$ is integrable. Given a linear and bounded operator $L: B \rightarrow B_{1}$ such that $B_{1}$ is a Banach space, then

$$
L\left(\int_{\mathbb{R}} f(t) d \mu(t)\right)=\int_{\mathbb{R}} L(f(t)) d \mu(t)
$$

Proof. Let $y=\int_{\mathbb{R}} f(t) d \mu(t)$. The function $L f: \mathbb{R} \rightarrow B_{1}$ is continous, moreover, $t \mapsto$ $\|L(f(t))\|$ is integrable, since

$$
\int_{\mathbb{R}}\|L(f(t))\| d \mu(t) \leq\|L\| \int_{\mathbb{R}}\|f(t)\| d \mu(t)<\infty .
$$

Let $z=\int_{\mathbb{R}} L(f(t)) d \mu(t)$. Given $\Lambda \in B_{1}^{*}, \Lambda L \in B^{*}$. Hence,

$$
\begin{aligned}
\Lambda z & =\Lambda \int_{\mathbb{R}} L(f(t)) d \mu(t) \\
& =\int_{\mathbb{R}} \Lambda L(f(t)) d \mu(t)
\end{aligned}
$$




$$
\begin{aligned}
& =\int_{\mathbb{R}}(\Lambda L)(f(t)) d \mu(t) \\
& =(\Lambda L) \int_{\mathbb{R}}(f(t)) d \mu(t) \\
& =(\Lambda L) y \\
& =\Lambda(L y) .
\end{aligned}
$$

Since $\Lambda$ is arbitrary, it follows that $z=L y$. Thus,

$$
L\left(\int_{\mathbb{R}} f(t) d \mu(t)\right)=\int_{\mathbb{R}} L(f(t)) d \mu(t) .
$$




\section{Chapter 3}

\section{Groupoids}

Groupoids can be understood as a generalization of groups where the unit is not unique and not every pair of elements can be multiplied. Each groupoid $G$ is endowed with two functions $r$ and $s$ from $G$ to the subset $G^{(0)}$ of units. We equip the groupoid with a topology such that $r, s$ are continuous.

If $G$ has some nice topological properties, we can define $C_{c}(G)$, the space of continuous and compactly supported complex functions on $G$. Moreover, we can endow this space with an involution and a product which is not necessarily commutative. Then we define a norm on $C_{c}(G)$ which depends on the *-representations of $C_{c}(G)$. Then the full groupoid $\mathrm{C}^{*}$-algebra, denoted $C^{*}(G)$ is the completion of $C_{c}(G)$ with respect to this norm.

Most definitions and results in this chapter can be found in [9].

\subsection{Introduction}

In this section we define groupoids and give some examples. The results in this section are taken from [9] and [20].

Definition 3.1.1. A groupoid is a set $G$ together with a subset $G^{(0)}$ (called units, unit space or objects), two surjective maps $r, s: G \rightarrow G^{(0)}$ (called range and source, respectively) and 
a law of composition

$$
(g, h) \in G^{(2)} \mapsto g h=g \cdot h \in G,
$$

where $G^{(2)}=\{(g, h) \in G \times G: s(g)=r(h)\}$ is called the set of composable elements or composable pairs.

A groupoid satisfies the following properties for $g, h, k \in G$ :

(i) $s(g h)=s(h)$ and $r(g h)=r(g)$ if $(g, h) \in G^{(2) \text {; }}$

(ii) $r(x)=s(x)$ if $x \in G^{(0)}$;

(iii) $g s(g)=g$ and $r(g) g=g$;

(iv) $(g h) k=g(h k)$ if $(g, h),(h, k) \in G^{(2)}$;

(v) $g$ has a two-sided inverse $g^{-1}$ such that $g g^{-1}=r(g)$ and $g^{-1} g=s(g)$.

The maps $(g, h) \in G^{(2)} \mapsto g h$ and $g \mapsto g^{-1}$ are called product and inverse, respectively.

We can interpret groupoids as a collection of arrows attached to points on a plane. Two arrows can be composed only if the end of the first arrow meets the start of the second. Units are points with the null vector and the inverse of an element is obtained by reversing the direction of the arrow. Figure 3.1 shows this idea.

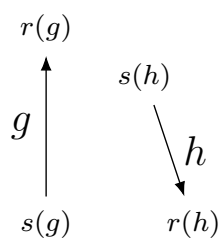

(a)

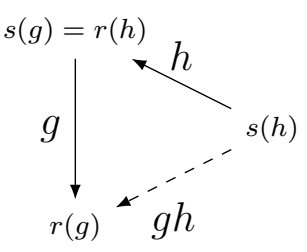

(b)

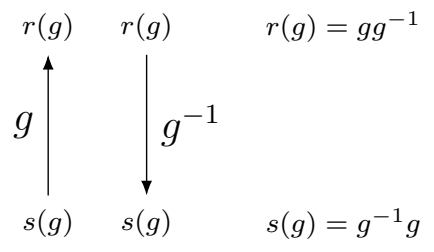

(c)

Figure 3.1: Groupoids can be seen as arrows on a plane. $s(g)$ and $r(g)$ denote the source and range of $g$. (a) $g$ and $h$ are not composable, since $s(g) \neq r(h)$; (b) The composition of $g$ and $h$ is $g h$; (c) $g^{-1}$ is the inverse of $g$. Note that $g^{-1} g=s(g)$ and $g g^{-1}=r(g)$. 
Example 3.1.2. Every group is a groupoid. Let $G$ be a group with unit $e$. Let $G^{(0)}=\{e\}$, $G^{(2)}=G \times G$ and define the range and source maps by $r(g)=s(g)=e$.

Since the range and source of each element is $e$ and $G$ is associative, one can easily show that properties (i)-(v) are satisfied.

Example 3.1.3. We show that a group action defines a groupoid. Let $G$ be a group with identity $e$ and $X$ a set. Recall that a group action [19] is a map $G \times X \rightarrow X$ denoted by $(g, x) \mapsto g x$, satisfying the following properties:

(i) $g(h x)=(g h) x \quad$ for $g, h \in G, x \in X$,

(ii) $e x=x \quad$ for $x \in X$.

If $G$ is an action, we say that $G$ acts on $X$.

The cartesian product $H=G \times X$ has a groupoid structure with unit space $H^{(0)}=\{e\} \times X$. The range and source maps are $s(g, x)=(e, x)$ and $r(g, x)=(e, g x)$ and the operations are defined by

$$
(g, h x)(h, x)=(g h, x) \quad \text { and } \quad(g, x)^{-1}=\left(g^{-1}, g x\right) .
$$

$H$ is a groupoid, called the action groupoid (or transformation groupoid [23]). In fact,

(i) $s((g, x)(h, y))=s(g, y)=(e, y)=s(h, y) \quad$ for $(g, x),(h, y)$ composable;

(ii) $r(e, x)=(e, e x)=(e, x)=s(e, x) \quad$ for $x \in X$;

(iii) Given $(g, x) \in H$,

$$
\begin{aligned}
& (g, x) s(g, x)=(g, x)(e, x)=(g e, x)=(g, x) \\
& r(g, x)(g, x)=(e, g x)(g, x)=(e g, x)=(g, x) ;
\end{aligned}
$$

(iv) Let $(g, x),(h, y),(k, z) \in H$ such that $((g, x),(h, y)),((h, y),(k, z)) \in H^{(2)}$. By hypoth- 
esis, $x=h y$ and $y=k z$. Hence, $x=h k z$. Then

$$
\begin{aligned}
{[(g, x)(h, y)](k, z) } & =(g h, y)(k, z) \\
& =(g h k, z) \\
& =(g, x)(h k, z) \\
& =(g, x)[(h, y)(k, z)] .
\end{aligned}
$$

(v) Given $(g, x) \in H$,

$$
\begin{aligned}
& (g, x)(g, x)^{-1}=(g, x)\left(g^{-1}, g x\right)=\left(g g^{-1}, g x\right)=(e, g x)=r(g, x) \\
& (g, x)^{-1}(g, x)=\left(g^{-1}, g x\right)(g, x)=\left(g^{-1} g, x\right)=(e, x)=s(g, x) .
\end{aligned}
$$

Example 3.1.4. Let $\sim$ be an equivalence relation on a set $X$. Let

$$
\begin{aligned}
G & =\{(x, y) \in X \times X: x \sim y\}, \\
G^{(0)} & =\{(x, x): x \in X\}, \text { and } \\
G^{(2)} & =\{((x, y),(y, z)): x \sim y, y \sim z\} .
\end{aligned}
$$

Define the range and source maps by $r(x, y)=(x, x)$ and $s(x, y)=(y, y)$. Let $(x, y)^{-1}=$ $(y, x)$ and $(x, y)(y, z)=(x, z)$. The inverse and multiplication maps are well-defined by the reflexivity and transitivity of $\sim$. Hence $G$ is a groupoid.

Remark 3.1.5. Note that [9] and [20] define groupoids differently. On the one hand, [9] introduces a groupoid as in Definition 3.1.1. On the other hand, Renuault [20] describes groupoids as follows:

A groupoid is a set $G$ endowed with a product map $(g, h) \mapsto g h: G^{(2)} \rightarrow G$, where $G^{(2)}$ is a subset of $G \times G$ called the set of composable pairs, and an inverse map $g \mapsto g^{-1}: G \rightarrow G$ such that the following relations are satisfied:

(i') $\left(g^{-1}\right)^{-1}=g$; 
(ii') If $(g, h),(h, k) \in G^{(2)}$, then $(g h, k),(g, h k) \in G^{(2)}$ and $(g h) k=g(h k)$;

(iii') $\left(g^{-1}, g\right) \in G^{(2)}$ and if $(g, h) \in G^{(2)}$, then $g^{-1}(g h)=h$;

(iv') $\left(g, g^{-1}\right) \in G^{(2)}$ and if $(h, g) \in G^{(2)}$, then $(h g) g^{-1}=h$.

Given $g \in G$, we define $r(g)=g g^{-1}$ and $s(g)=g^{-1} g$. The unit space is defined by $G^{(0)}=s(G)=r(G)$.

These definitions are equivalent.

First, suppose $G$ is a groupoid as in Definition 3.1.1. Note that $r(x)=s(x)=x$ for each $x \in G^{(0)}$. In fact, $r(x)=s(x)$ by property (ii). Since $s: G \rightarrow G^{(0)}$ is surjective, there exists $g \in G$ such that $x=s(g)=g^{-1} g$. Hence,

$$
s(x)=s(s(g))=s\left(g^{-1} g\right)=s(g)=x .
$$

Now we prove properties (i')-(iv').

(i') $\left(g^{-1}\right)^{-1}=g$

Since $s(g)=g^{-1} g$ and $s(s(g))=r(s(g))$, we have

$$
\begin{aligned}
& s(s(g))=s(g)=g^{-1} g, \\
& r(s(g))=r\left(g^{-1} g\right)=r\left(g^{-1}\right)=g^{-1}\left(g^{-1}\right)^{-1} .
\end{aligned}
$$

Then $g^{-1} g=g^{-1}\left(g^{-1}\right)^{-1}$ and therefore $g=\left(g^{-1}\right)^{-1}$.

(ii') $(g h) k=g(h k)$

This holds by property (iv).

(iii') $g^{-1}(g h)=h$

Note that $s(g)=r(h)$. Then

$$
g^{-1}(g h)=\left(g^{-1} g\right) h=s(g) h=r(h) h=h .
$$


(iv') $(h g) g^{-1}=h$

Note that $s(h)=r(g)$. Then

$$
(h g) g^{-1}=h\left(g g^{-1}\right)=h r(g)=h s(h)=h .
$$

Then $G$ is a groupoid as defined in [20].

Conversely, assume that $G$ is a groupoid as in [20].

First we show that $G^{(2)}=\{(g, h): s(g)=r(h)\}$. Suppose $(g, h) \in G^{(2)}$. Then,

$$
\begin{aligned}
g^{-1}(g h) & =h \quad \text { by (iii'), } \\
{\left[g^{-1}(g h)\right] h^{-1} } & =h h^{-1} \quad \text { by (iv'), } \\
{\left[\left(g^{-1} g\right) h\right] h^{-1} } & =h h^{-1} \\
g^{-1} g & =h h^{-1} \\
s(g) & =r(h) .
\end{aligned}
$$

Suppose $g, h \in G$ are such that $s(g)=r(g)$. Then

$$
\begin{aligned}
\left(h, h^{-1}\right),\left(h^{-1}, h\right) \in G^{(2)} \quad \text { by }(i i i '),\left(\mathrm{iv}^{\prime}\right), \\
\Rightarrow\left(h h^{-1}, h\right) \in G^{(2)} \\
\Rightarrow(r(h), h) \in G^{(2)} \\
\Rightarrow(s(g), h) \in G^{(2)} \\
\Rightarrow\left(g^{-1} g, h\right) \in G^{(2)} \\
\Rightarrow\left(g^{-1} g, h\right) \in G^{(2)} .
\end{aligned}
$$


On the other hand,

$$
\begin{array}{r}
\left(g, g^{-1}\right),\left(g^{-1}, g\right) \in G^{(2)} \quad \text { by (iii') },\left(\text { iv'}^{\prime}\right), \\
\Rightarrow\left(g, g^{-1} g\right) \in G^{(2)} .
\end{array}
$$

Then $\left(g, g^{-1} g\right),\left(g^{-1} g, h\right) \in G^{(2)}$. Therefore $(g, h) \in G^{(2)}$.

Now we prove properties (i)-(v).

(i) $s(g h)=s(h), r(g h)=r(g)$

By assumption, $(g, h) \in G^{(2)}$. Also, $\left(h, h^{-1}\right) \in G^{(2)}$ by (iii). Then $s(h)=r\left(h^{-1}\right)$ and $\left(g h, h^{-1}\right) \in G^{(2)}$. This implies $s(g h)=r\left(h^{-1}\right)=s(h)$.

The proof of $r(g h)=r(g)$ is analogous.

(ii) $r(x)=s(x)$ if $x \in G^{(0)}$

Given $x \in G^{(0)}$, there exists $g \in G$ such that $x=r(g)=g g^{-1}$. Then

$$
s(x)=s\left(g g^{-1}\right)=s\left(g^{-1}\right)=r(g)=x .
$$

Note that $s\left(g g^{-1}\right)=s\left(g^{-1}\right)$ by $(\mathrm{i})$.

(iii) $g s(g)=g$ and $r(g) g=g$

$$
\begin{aligned}
& g s(g)=g\left(g^{-1} g\right)=\left(g^{-1}\right)^{-1}\left(g^{-1} g\right)=g, \\
& r(g) g=\left(g g^{-1}\right) g=\left(g g^{-1}\right)\left(g^{-1}\right)^{-1}=g .
\end{aligned}
$$

(iv) $(g h) k=g(h k)$

This is equivalent to (ii')

(v) $r(g)=g g^{-1}, g^{-1} g=s(g)$

This follows from the definition of $r, s$. 
Therefore the definitions are equivalent.

Remark 3.1.6. Given $A, B$ subsets of a groupoid $G$, one may form the following subsets of G:

$$
A^{-1}=\left\{g \in G: g^{-1} \in A\right\}, A B=\{g h \in G: g \in A, h \in B\}
$$

Given $x, y \in G^{(0)}$ :

$$
G^{x}=r^{-1}(x), G_{y}=s^{-1}(y), \quad \text { and } G_{y}^{x}=G^{x} \cap G_{y}
$$

$G^{x}$ (resp. $\left.G_{y}\right)$ is called the $r$-fiber of $G$ over $x$ (resp. s-fiber of $G$ over $y$ ) as in [7].

Note that $G_{x}^{x}$ is a group. It is called the isotropy group at $x$. In fact,

(i) $g h \in G_{x}^{x}$ for $g, h \in G_{x}^{x}$;

(ii) $g g^{-1}=g^{-1} g=x$ for $g \in G_{x}^{x}$. Hence $x$ is the unity of $G_{x}^{x}$;

(iii) the product in $G_{x}^{x}$ associative.

Notation 3.1.7. Unless otherwise specified, we will use the following notation in this thesis: $G$ denotes a groupoid; its units are denoted by the letters $x, y, z ; g, h$ are elements in $G$. Subsets of $G$ may be written as the uppercase letters $U, V$. The letters may be indexed or marked with an accent or symbol.

\subsection{Topological Groupoids}

If $G$ is a groupoid endowed with a topology, it is useful that its operations have interesting topological properties. We define the notion of topological groupoid, where its operations are continuous. We also define étale groupoids, where the range and source maps are local homeomorphisms. This section is based on [9] and [20]. 
Definition 3.2.1. A topological groupoid is a groupoid $G$ with a topology such that $G^{(2)}$ has the induced topology from $G \times G$, and both the product and inverse maps are continuous.

Remark 3.2.2. Let $G$ be a topological groupoid. Since $r$ and $s$ are defined by $r(g)=g g^{-1}$ and $s(g)=g^{-1} g$, it follows that these functions are continuous.

Now we define the notion of étale groupoid. The main results in this thesis assume the groupoid has this property.

Definition 3.2.3. A topological groupoid is étale if the maps $r$ and $s$ are local homeomorphisms.

Example 3.2.4. Every discrete groupoid $G$ is étale. In fact, for every $g \in G$, the subsets $\{g\},\{r(g)\},\{s(g)\}$ are open in $G$. Moreover, the maps $\left.r\right|_{\{g\}}:\{g\} \rightarrow\{r(g)\},\left.s\right|_{\{g\}}:\{g\} \rightarrow$ $\{s(g)\}$ are homeomorphimsms. In particular, every discrete group is étale.

Example 3.2.5. Let $X$ be a topological space and let $r, s: X \rightarrow X$ be identity maps. Moreover, defined for each $x \in X, x x=x$ and $x^{-1}=x$. Then $X$ is an étale groupoid because $r, s$ are homeomorphimsms.

Another example of étale groupoid is the transformation groupoid $G \times X$ when the group $G$ is discrete. We prove this in the following lemma:

Lemma 3.2.6. Let $G$ be a group endowed with a topology. Let $X$ be a topological space and fix a continuous group action $G \times X \rightarrow X$. Suppose $G \times X$ is the action groupoid as in Example 3.1 .3 and equip this space with the product topology. Then $G \times X$ is étale if, and only if, $G$ is discrete.

Proof. - Suppose $G$ is not discrete.

There exists $g \in G$ such that for each neighborhood $U$ of $g, U \backslash\{g\} \neq \varnothing$.

Fix $x \in X$ and let $V$ be an arbitrary open neighborhood of $x$. Let $U$ be an open neighborhood of $g$. Then there exists $h \neq g$ such that $h \in U$. Then $(g, x),(h, x) \in U \times V$ 
and $s(g, x)=s(h, x)=(e, x)$. Since $U, V$ are arbitrary, it follows that $s$ is not a local homeomorphimsm. Therefore, $G$ is not étale.

- Now suppose that $G$ is discrete

Then the product and inverse maps on the group $G$ are continuous. Note that product and inverse maps on the groupoid are continuous because they are compositions of continuous functions. Then $G \times X$ is a topological groupoid.

For each $g \in G$ the map $X \mapsto X$ defined by $x \mapsto g x$ is a homeomorphimsm with inverse $x \mapsto g^{-1} x$. Then, for every open set $U \subset X$, the set $g U=\{g x: x \in U\}$ is open in $X$.

Now we show that $G \times X$ is étale. Let $(g, x) \in G \times X, U$ a neighborhood of $x \in X$. Then $\{g\} \times U$ is an open neighborhood of $(g, x)$. Then

$$
\begin{aligned}
& r(\{g\} \times U)=\{(e, g x): x \in U\}=\{e\} \times g U, \\
& s(\{g\} \times U)=\{(e, x): x \in U\}=\{e\} \times U .
\end{aligned}
$$

Then $r(\{g\} \times U), s(\{g\} \times U)$ are open sets in $G \times X$.

The function $\left.s\right|_{\{g\} \times U}$ is injective. The function $x \in U \mapsto g x$ is injective, then $r$ is injective on $\{g\} \times U$. Since $g$ and $U$ are arbitrary, it follows that $r, s$ are open bisections. Therefore, $G \times X$ is étale.

Definition 3.2.7. An open subset $\mathcal{U}$ of an étale groupoid is an open bisection of $G$ if $r(\mathcal{U}), s(\mathcal{U})$ are open in $G^{(0)}$, and $\left.r\right|_{\mathcal{U}}: \mathcal{U} \rightarrow r(\mathcal{U})$ and $\left.s\right|_{\mathcal{U}}: \mathcal{U} \rightarrow s(\mathcal{U})$ are homeomorphisms.

Notation 3.2.8. We will usually denote an open bisection by the cursive letter $\mathcal{U}$. This letter may be indexed or marked with an accent or symbol.

Remark 3.2.9. Many times throughout the thesis, we will evaluate sums which take into account values $f(g)$ such that $g$ ranges over $G_{x}$ or $G^{x}$, assuming $f \in C_{c}(G)$. However, if this 
function is supported on an open bisection, we can consider only one term in the sum. This element is usually denoted $h_{x}\left(\operatorname{resp} . h^{x}\right)$ and $h_{x} \in G_{x} \cap \mathcal{U}\left(\operatorname{resp} . h^{x} \in G^{x} \cap \mathcal{U}\right)$.

Later we prove that every $f \in C_{c}(G)$ can be written as a finite sum of continuous functions supported on open bisections. Hence, in many cases, we can assume $f$ is supported on an open bisection without loss of generality.

Proposition 3.2.10. Let $G$ be an étale groupoid. The set of open bisections of $G$ forms an open base for the topology of $G$.

Proof. Let $U$ be an open set of $G$. We will show that for every $g \in U$ there exists an open bisection $\mathcal{U}_{g}$ such that $g \in \mathcal{U}_{g} \subset U$. In fact, let $g \in U$. Since $G$ is étale, $r, s$ are local homeomorphimsms. Then there exist $R_{g}, S_{g}$ open neighborhoods of $g$ such that $r\left(R_{g}\right)$ and $s\left(S_{g}\right)$ are open in $G^{(0)}$, and $\left.r\right|_{R_{g}}: R_{g} \rightarrow r\left(R_{g}\right),\left.s\right|_{S_{g}}: S_{g} \rightarrow s\left(S_{g}\right)$ are homeomorphisms.

Let $\mathcal{U}_{g}=R_{g} \cap S_{g} \cap U . \quad r\left(\mathcal{U}_{g}\right)$ is open in $r\left(R_{g}\right)$, then $r\left(\mathcal{U}_{g}\right)$ is open in $G^{(0)}$. Hence, $\left.r\right|_{\mathcal{U}_{g}}: \mathcal{U}_{g} \rightarrow r\left(\mathcal{U}_{g}\right)$ is a homeomorphism. Analogously $\left.s\right|_{\mathcal{U}_{g}}: \mathcal{U}_{g} \rightarrow s\left(\mathcal{U}_{g}\right)$ is a homeomorphism.

Therefore, for every open set $U$, we have $U=\bigcup_{g \in U} \mathcal{U}_{g}$.

Proposition 3.2.11. If $G$ is an étale groupoid, then the subspace topology of $G^{x}$ and $G_{x}$ is equivalent to the discrete topology for all $x \in G^{(0)}$. Furthermore, if $G$ is second countable, then $G^{x}$ and $G_{x}$ have a countable number of elements.

Proof. Let $g \in G^{x}$. There exists an open bisection $\mathcal{U}_{g}$ containing $g$. We show that $\mathcal{U}_{g} \cap G^{x}=$ $\{g\}$. Suppose there exists $h \neq g$ such that $h \in \mathcal{U}_{g} \cap G^{x}$. Then $r(h)=x$. Contradiction, since $r$ is injective on $\mathcal{U}_{g}$. Hence $\{g\}$ is open in $G^{x}$. Therefore $G^{x}$ is endowed with the discrete topology.

Assume $G$ is second countable. Then $G^{x}$ is second countable. Since the sets $\{g\}, g \in G^{x}$, form a family of disjoint open sets in $G^{x}$, it follows that $G^{x}$ is countable. The proof for $G_{x}$ is analogous.

Proposition 3.2.12. If $G$ is a locally compact Hausdorff étale groupoid, then $G^{(0)}$ is a clopen subset of $G$. We assume $G^{(0)}$ is endowed with the subspace topology. 
Proof. We divide the proof in two parts.

- $G^{(0)}$ is closed

Let $x_{i}$ be a net in $G^{(0)}$ converging to $x \in G$. The function $r$ is continuous, then $r\left(x_{i}\right) \rightarrow r(x)$. As $x_{i} \in G^{(0)}$, we have $r\left(x_{i}\right)=x_{i}$. Hence $x=r(x) \in G^{(0)}$. Therefore $G^{(0)}$ is closed.

- $G^{(0)}$ is open

Let $x \in G^{(0)}$. Let $\mathcal{U} \subset G$ be an open bisection containing $x$. Let $V=G^{(0)} \cap \mathcal{U}$. Then $V$ is an open neighborhood in $G^{(0)}$ of $x$. Moreover, $V \subset r(\mathcal{U})$, since $r(y)=y$ for every $y \in V$.

Since $\left.r\right|_{\mathcal{U}}: \mathcal{U} \rightarrow r(\mathcal{U})$ is a homeomorphimsm, $\left.r\right|_{\mathcal{U}} ^{-1}(V)=V$. Then $V$ is open in $G$. Therefore $G^{(0)}$ is open.

Let $G^{\prime}=\cup_{x \in G^{(0)}} G_{x}^{x}$, called the isotropy bundle. The following lemma shows that $G^{\prime}$ is closed.

Lemma 3.2.13. Let $G$ be a locally compact Hausdorff second countable étale groupoid. Given $g \in G$ such that $r(g) \neq s(g)$, there exists an open bisection $\mathcal{U}$ including $g$ such that $r(\mathcal{U}) \cap s(\mathcal{U})=\varnothing$. Moreover, $G^{\prime} \cap \mathcal{U}=\varnothing$. In particular, $G^{\prime}$ is closed.

Proof. Suppose this lemma is false. Then there exists $g \in G \backslash G^{\prime}$ such that for every open bisection $\mathcal{U}$ including $g$, we have

$$
r(\mathcal{U}) \cap s(\mathcal{U}) \neq \varnothing
$$

Since $G$ is second countable and étale, we can choose a countable family $\left\{\mathcal{U}_{n}\right\}$ of open bisections containing $g$ such that every neighborhood of $g$ contains at least one $\mathcal{U}_{n}$. Hence, 
for every $n$ there are $g_{n}, h_{n} \in \mathcal{U}_{n}$ satisfying

$$
r\left(g_{n}\right)=s\left(h_{n}\right)
$$

By definition, both sequences $\left\{g_{n}\right\}_{n \in \mathbb{N}},\left\{h_{n}\right\}_{n \in \mathbb{N}}$ converge to $g$ as $n \rightarrow \infty$. Then, by continuity of $r, s$, we have $r\left(g_{n}\right) \rightarrow r(g)$ and $s\left(h_{n}\right) \rightarrow s(g)$. However, from (3.1), we have $s\left(h_{n}\right) \rightarrow r(g)$. Hence $r(g)=s(g)$. This leads to a contradiction because we assumed $g \notin G^{\prime}$.

Therefore we can choose $\mathcal{U}$ satisfying $r(\mathcal{U}) \cap s(\mathcal{U})=\varnothing$. Moreover, $G^{\prime} \cap \mathcal{U}=\varnothing$. Since $g \in G \backslash G^{\prime}$ is arbitrary, it follows that $G^{\prime}$ is closed.

Remark 3.2.14. Let $G$ be a groupoid and $V \subset G^{(0)}$. We define $\left.G\right|_{V}=G \cap r^{-1}(V) \cap s^{-1}(V)$. Note that $\left.G\right|_{V}$ is a groupoid. If $G$ is a topological groupoid, then $\left.G\right|_{V}$ is also a topological groupoid. Analogously, if $G$ is étale, so is $\left.G\right|_{V}$.

\subsection{Groupoid C*-Algebras}

Now we define the full groupoid $\mathrm{C}^{*}$-algebra and prove some properties of this $\mathrm{C}^{*}$-algebra. The results in this section can be found in [5], [9] and [23].

Let $G$ be a locally compact second countable Hausdorff étale groupoid. Denote $C_{c}(G)$ by

$$
C_{c}(G)=\{f: G \rightarrow \mathbb{C}: f \text { is continuous and } \operatorname{supp}(f) \text { is compact }\} .
$$

Recall that the support of $f$ is defined by $\operatorname{supp}(f)=\overline{\{g \in G: f(g) \neq 0\}}$. We define the convolution and involution operations on $C_{c}(G)$ by

$$
\left(f_{1} \cdot f_{2}\right)(g)=\sum_{g_{1} g_{2}=g} f_{1}\left(g_{1}\right) f_{2}\left(g_{2}\right) \quad \text { and } \quad f^{*}(g)=\overline{f\left(g^{-1}\right)}
$$

Example 3.3.1. Let $n$ be a positive integer and define the groupoid $G=\{(i, j): i, j=$ 
$1, \ldots, n\}$ such that

$$
\begin{aligned}
& G^{(0)}=\{(i, i): i=1, \ldots, n\} \\
& G^{(2)}=\{((i, k),(k, j)): i, j, k=1, \ldots, n\},
\end{aligned}
$$

and define the operations

$$
(i, k)(k, j)=(i, j) \text { and }(i, j)^{-1}=(j, i)
$$

Equip $G$ with the discrete topology. Then $G$ is locally compact Hausdorff. Moreover, $G$ is étale by Example 3.2.4.

Note that there is a bijection from $C_{c}(G)$ to $M_{n}(\mathbb{C})$ given by $f \mapsto F$ such that $F_{i, j}=f(i, j)$. Moreover, we can identify $C_{c}(G)$ with $M_{n}(\mathbb{C})$. In fact, let $f^{(1)}, f^{(2)} \in C_{c}(G)$. Assume $A$ is a matrix corresponding to $f^{(1)} \cdot f^{(2)}$. Then, for every $i, j=1, \ldots, n$,

$$
A_{i, j}=\left(f^{(1)} \cdot f^{(2)}\right)(i, j)=\sum_{k=1}^{n} f^{(1)}(i, k) f^{(2)}(k, j)=\sum_{k=1}^{n} F_{i, k}^{(1)} F_{k, j}^{(2)}=\left(F^{(1)} F^{(2)}\right)_{i, j},
$$

then $A=F^{(1)} F^{(2)}$.

Now assume $F \in M_{n}(\mathbb{C})$ corresponds to $f \in C_{c}(G)$. Then $F^{*}$ corresponds to $f^{*}$. In fact, for $i, j=1, \ldots, n$,

$$
F_{i, j}^{*}=\overline{F_{j, i}}=\overline{f(j, i)}=\overline{f\left((i, j)^{-1}\right)}=f^{*}(i, j) .
$$

Therefore we can identify $M_{n}(\mathbb{C})$ with $C_{c}(G)$. Moreover, $C_{c}(G)$ is not commutative.

Notation 3.3.2. We usually denote a function in $C_{c}(G)$ by $f$. Note that, for an open subset $U \subset G$, every function in $C_{c}(U)$ can be extended uniquely to a function in $C_{c}(G)$ whose support lies in $U$. Thus, for every open set $U \subset G$, we will denote without loss of 
generality,

$$
C_{c}(U)=\left\{f \in C_{c}(G): \operatorname{supp}(f) \subset U\right\}
$$

Thus $C_{c}(U)$ is a subspace of $C_{c}(G)$.

The letter $h$ sometimes denotes elements in $C_{c}\left(G^{(0)}\right)$. However, $h$ is also used to indicate elements in $G$.

Given $f_{1}, f_{2} \in C_{c}(G), f_{1} f_{2}$ denotes the pointwise product of these functions, while $f_{1} \cdot f_{2}$ denotes the convolution product.

Lemma 3.3.3. Let $G$ be a locally compact second countable Hausdorff étale groupoid. Given $f_{1}, f_{2} \in C_{c}(G), g \in G$,

$$
\begin{aligned}
\left(f_{1} \cdot f_{2}\right)(g) & =\sum_{h \in G_{s(g)}} f_{1}\left(g h^{-1}\right) f_{2}(h) \\
& =\sum_{h \in G^{r(g)}} f_{1}(h) f_{2}\left(h^{-1} g\right) .
\end{aligned}
$$

Proof. Let $g_{1} g_{2} \in G$ such that $g_{1} g_{2}=g$. This is equivalent to $g_{1}=g g_{2}^{-1}$. This equation holds only if $g_{2} \in G^{s(g)}$. Therefore, for every $h \in G^{s(g)}$ we can choose $g_{2}=h$ and $g_{1}=g h^{-1}$. Then,

$$
\left(f_{1} \cdot f_{2}\right)(g)=\sum_{h \in G^{r(g)}} f_{1}\left(g h^{-1}\right) f_{2}(h)
$$

This sum is finite for every $g$, since $G^{r(g)}$ is countable and $f_{2}$ is compactly supported, then the set of elements $h \in G^{r(g)}$ such that $f_{2}(h) \neq 0$ is finite. The proof for 3.4 is analogous.

Lemma 3.3.4. Let $f \in C_{c}(G), h \in C_{c}\left(G^{(0)}\right), g \in G$. Then

$$
(h \cdot f)(g)=h(r(g)) f(g), \quad \text { and } \quad(f \cdot h)(g)=f(g) h(s(g)) .
$$


Proof. It follows from Lemma 3.3.3 that

$$
(h \cdot f)(g)=\sum_{k \in G^{r(g)}} h(k) f\left(k^{-1} g\right)
$$

Suppose $h(k) \neq 0$ for some $k \in G^{r(g)}$. Then $k \in G^{(0)}$ and $r(k)=r(g)$. Hence $k=r(g)$ and therefore,

$$
(h \cdot f)(g)=h(r(g)) f\left(r(g)^{-1} g\right)=h(r(g)) f(r(g) g)=h(r(g)) f(g) .
$$

The proof for $f \cdot h$ is analogous.

Now we show that every function in $C_{c}(G)$ can be decomposed as a sum of continuous compactly supported functions whose support are included in open bisections. This result will be used many times in the thesis because many results are easier to prove when the function is supported on an open bisection.

Lemma 3.3.5. Let $G$ be a locally compact second countable étale Hausdorff groupoid. Given $f \in C_{c}(G)$, there are $\mathcal{U}_{1}, \ldots, \mathcal{U}_{n}$ open bisections and $f_{1}, \ldots, f_{n}$ functions such that $f=f_{1}+\ldots+f_{n}$ and each $f_{i} \in C_{c}\left(\mathcal{U}_{i}\right)$. Moreover, if $f$ is non-negative, we can choose each $f_{i}$ to be non-negative.

Proof. Let $f \in C_{c}(G)$ with support $K$. From Proposition 3.2.10 the set of open bisections forms an open base for $G$. Then there exists a finite cover $\mathcal{U}_{1}, \ldots \mathcal{U}_{n}$ of $K$ such that each $\mathcal{U}_{i}$ is an open bisection.

Let $\mathcal{U}_{n+1}=G \backslash K$. Then $\left\{\mathcal{U}_{i}\right\}_{i=1}^{n+1}$ is an open cover of $G$. Let $\left\{\alpha_{i}\right\}_{i=1}^{n+1}$ be the partition of unit subordinate to the the open cover $\left\{U_{i}\right\}_{i=1}^{n+1}$. Note that $f \alpha_{n+1}=0$ since $\alpha_{n+1}$ is supported on $\mathcal{U}_{n+1} \backslash K$. Then,

$$
f=\sum_{i=1}^{n+1} f \alpha_{i}=\sum_{i=1}^{n} f \alpha_{i} .
$$


Define $f_{i}=f \alpha_{i}$ for $i=1, \ldots, n$. By definition of $\alpha_{i}$, each $f_{i} \in C_{c}\left(\mathcal{U}_{i}\right)$. Moreover, since $\alpha_{i}$ assumes values in the interval $[0,1]$, if $f$ is non-negative, it follows that each $f_{i}$ is non-negative.

Lemma 3.3.6. Let $G$ be a locally compact Hausdorff second countable étale groupoid. If $\mathcal{U}, \mathcal{V} \subset G$ are open bisections, then

$$
\mathcal{U V}=\left\{g h: g \in \mathcal{U}, h \in \mathcal{V},(g, h) \in G^{(2)}\right\}
$$

is an open bisection.

Proof. Before we prove $\mathcal{U} \mathcal{V}$ is an open bisection, we will show that we can assume $s(\mathcal{U})=r(\mathcal{V})$ without loss of generality. Let $W=s(\mathcal{U}) \cap r(\mathcal{V})$. Then $W$ is an open set in $G^{(0)}$ because $\mathcal{U}$, $\mathcal{V}$ are open bisections.

Let $\mathcal{U}_{0}=\left.s\right|_{\mathcal{U}} ^{-1}(W)$ and $\mathcal{V}_{0}=\left.r\right|_{\mathcal{V}} ^{-1}(W)$. Both $\mathcal{U}_{0}, \mathcal{V}_{0}$ are open bisections, since they are open subsets of open bisections. Moreover, we have

$$
s\left(\mathcal{U}_{0}\right)=\left.s \circ s\right|_{\mathcal{U}} ^{-1}(W)=W=\left.r \circ r\right|_{\mathcal{V}} ^{-1}(W)=r\left(\mathcal{V}_{0}\right)
$$

Now we show that $\mathcal{U} \times \mathcal{V} \cap G^{(2)}=\mathcal{U}_{0} \times \mathcal{V}_{0} \cap G^{(2)}$. In fact, given $(g, h) \in \mathcal{U} \times \mathcal{V} \cap G^{(2)}$, we have $g \in \mathcal{U}, h \in \mathcal{V}$ and $s(g)=r(h)$. If we define $x=s(g)$, then $x \in W$. Moreover, $g=\left.s\right|_{\mathcal{U}} ^{-1}(x)$, which implies $g \in \mathcal{U}_{0}$. Analogously, $h \in \mathcal{V}_{0}$. Then $(g, h) \in \mathcal{U}_{0} \times \mathcal{V}_{0} \cap G^{(2)}$. Therefore $\mathcal{U} \times \mathcal{V} \cap G^{(2)} \subset \mathcal{U}_{0} \times \mathcal{V}_{0} \cap G^{(2)}$. Since $\mathcal{U}_{0} \subset \mathcal{U}$ and $\mathcal{V}_{0} \subset \mathcal{V}$, we have $\mathcal{U} \times \mathcal{V} \cap G^{(2)}=\mathcal{U}_{0} \times \mathcal{V}_{0} \cap G^{(2)}$

By definition $\mathcal{U} \mathcal{V}$, we have

$$
\begin{aligned}
\mathcal{U V} & =\left\{g h: g \in \mathcal{U}, h \in \mathcal{V},(g, h) \in G^{(2)}\right\} \\
& =\left\{g h:(g, h) \in \mathcal{U} \times \mathcal{V} \cap G^{(2)}\right\} \\
& =\left\{g h:(g, h) \in \mathcal{U}_{0} \times \mathcal{V}_{0} \cap G^{(2)}\right\} \\
& =\mathcal{U}_{0} \mathcal{V}_{0}
\end{aligned}
$$


Therefore we can assume $s(\mathcal{U})=r(\mathcal{V})$ without loss of generality.

Now we prove $\mathcal{U V}$ is an open bisection. Assume $s(\mathcal{U})=r(\mathcal{V})$. Let $\phi: \mathcal{U} \rightarrow \mathcal{V}$ be the homeomorphism defined by $\phi=\left.\left.r\right|_{\mathcal{V}} ^{-1} \circ s\right|_{\mathcal{U}}$.

Define the map $f$ from $\mathcal{U}$ to $\mathcal{U} \times \mathcal{V}$ by $f(g)=(g, \phi(g))$. The image $f(\mathcal{U})$ is included in $\mathcal{U} \times \mathcal{V} \cap G^{(2)}$, since

$$
r(\phi(g))=r\left(\left.r\right|_{\mathcal{V}} ^{-1} \circ s(g)\right)=s(g)
$$

We claim $f(\mathcal{U})=\mathcal{U} \times \mathcal{V} \cap G^{(2)}$. Suppose $(g, h) \in \mathcal{U} \times \mathcal{V} \cap G^{(2)}$. Then $s(g)=r(g), g \in \mathcal{U}$, $h \in \mathcal{V}$. Hence

$$
h=\left.\left.r\right|_{\mathcal{V}} ^{-1} \circ s\right|_{\mathcal{U}}(g)=\phi(g)
$$

Thus $(g, h)=(g, \phi(g))=f(g)$. Therefore $f(\mathcal{U})=\mathcal{U} \times \mathcal{V} \cap G^{(2)}$.

By definition of $f$, we have that $f$ is injective, thus. We will show that $f$ is a homeomorphism. Let $\pi: \mathcal{U} \times \mathcal{V} \cap G^{(2)} \rightarrow \mathcal{U}$ be the projection onto the first coordinate. $\pi$ is continuous by definition. So we will show that $\pi$ is the inverse of $f$.

Given $g \in \mathcal{U}$,

$$
\pi \circ f(g)=\pi(g, \phi(g))=g .
$$

Given $(g, h) \in \mathcal{U} \times \mathcal{V} \cap G^{(2)}$, we have $(g, h)=(g, \phi(g))=f(g)$ since $f$ is a bijection. Then

$$
(f \circ \pi)(g, h)=f(g)=(g, \phi(g))=(g, h) .
$$

Therefore $\pi$ is the inverse of $f$ and $f$ is a homeomorphism. Hence the set $\mathcal{U} \times \mathcal{V} \cap G^{(2)}$ is open in $\mathcal{U} \times \mathcal{V}$. 
Now we can consider the product $p: \mathcal{U} \times \mathcal{V} \cap G^{(2)} \rightarrow \mathcal{U V}$ and observe that

$$
\left.r\right|_{\mathcal{U V}} \circ p=\left.r\right|_{\mathcal{U}} \circ \pi
$$

In fact, given $(g, h) \in \mathcal{U} \times \mathcal{V} \cap G^{(2)}$,

$$
\left.r\right|_{\mathcal{U V}} \circ p(g, h)=r(g h)=r(g)=\left.r\right|_{\mathcal{U}} \circ \pi(g, h) .
$$

Equation (3.5) shows that $\left.r\right|_{\mathcal{U V}} \circ p$ is a homeomorphism. Moreover, we conclude that $p$ is surjective and $\left.r\right|_{\mathcal{U V}}$ is injective.

In addition, $p$ is injective because if $p\left(g_{1}, h_{1}\right)=p\left(g_{2} h_{2}\right)$, we have by (3.5) the following result,

$$
\begin{aligned}
\left.r\right|_{\mathcal{U}} \circ \pi\left(g_{1}, h_{1}\right) & =\left.r\right|_{\mathcal{U}} \circ \pi\left(g_{2}, h_{2}\right) \\
\left.r\right|_{\mathcal{U}}\left(g_{1}\right) & =\left.r\right|_{\mathcal{U}}\left(g_{1}\right) \\
g_{1} & =g_{2}, \quad \text { since } \mathcal{U} \text { is an open bisection, } \\
r\left(h_{1}\right) & =r\left(h_{2}\right) \quad \text { because }\left(g_{1}, h_{1}\right),\left(g_{2}, h_{2}\right) \in \mathcal{U} \times \mathcal{V} \cap G^{(2)}, \\
h_{1} & =h_{2} \quad \text { since } \mathcal{V} \text { is an open bisection. }
\end{aligned}
$$

Therefore $p$ is injective. Hence $\left.r\right|_{\mathcal{U V}}, p$ are continuous bijections such that their composition is a homeomorphism. Therefore $p: \mathcal{U} \times \mathcal{V} \cap G^{(2)} \rightarrow \mathcal{U V}$ is a homeomorphism.

Since $\mathcal{U} \times \mathcal{V} \cap G^{(2)}$ is an open set, so is $\mathcal{U V}$. We already proved that $\left.r\right|_{\mathcal{U V}}$ is injective. The proof for $\left.s\right|_{\mathcal{U V}}$ is analogous. Therefore, $\mathcal{U V}$ is an open bisection.

Lemma 3.3.7. Let $G$ be a locally compact Hausdorff second countable étale groupoid. If $\mathcal{U} \subset G$ is an open bisection, then $\mathcal{U}^{-1}=\left\{g^{-1}: g \in \mathcal{U}\right\}$ is an open bisection.

Proof. Let $\iota: G \rightarrow G$ be the inverse map. $\iota$ is continuous and $\iota \circ \iota$ is the identity. Then $\mathcal{U}^{-1}=\iota(\mathcal{U})$ is open. 
Let $g_{1}, g_{2} \in \mathcal{U}^{-1}$ such that $r\left(g_{1}\right)=r\left(g_{2}\right)$. There exist $h_{1}, h_{2} \in \mathcal{U}$ such that $g_{i}=h_{i}^{-1}$, $i=1,2$. Then

$$
s\left(h_{1}\right)=r\left(g_{1}\right)=r\left(g_{2}\right)=s\left(h_{2}\right)
$$

Since $\mathcal{U}$ is an open bisection, we have $h_{1}=h_{2}$. Then $g_{1}=g_{2}$. The proof for $s$ is analogous. Therefore $\mathcal{U}^{-1}$ is an open bisection.

Lemma 3.3.8. Let $G$ be a locally compact Hausdorff second countable étale groupoid.

(i) Given $\mathcal{U}_{1}, \mathcal{U}_{2}$ open bisections, $f_{1} \in C_{c}\left(\mathcal{U}_{1}\right), f_{2} \in C_{c}\left(\mathcal{U}_{2}\right)$, then $f_{1} \cdot f_{2} \in C_{c}\left(\mathcal{U}_{1} \mathcal{U}_{2}\right)$.

(ii) If $\mathcal{U}$ is an open bisection and $f \in C_{c}(\mathcal{U})$, we have $f^{*} \in C_{c}\left(\mathcal{U}^{-1}\right)$.

Proof. (i) Note that $\mathcal{U}_{1} \mathcal{U}_{2}$ is an open bisection by Lemma 3.3.6.

Let $g \notin \mathcal{U}_{1} \mathcal{U}_{2}$. Then $f_{1} \cdot f_{2}(g)=0$ since $f(g) \neq 0$ implies that there are $g_{1} \in \mathcal{U}_{1}, g_{2} \in \mathcal{U}_{2}$ satisfying $g_{1} g_{2}=g$. Therefore the support of $f_{1} \cdot f_{2}$ lies in $\mathcal{U}_{1} \mathcal{U}_{2}$.

Since $\mathcal{U}_{1} \mathcal{U}_{2}$ is an open bisection, the maps $u_{1}, u_{2}$ are homeomorphisms where $u_{1}$ : $\mathcal{U}_{1} \mathcal{U}_{2} \rightarrow \mathcal{U}_{1}$ is defined by $u_{1}=\left.r\right|_{\mathcal{U}_{1}} ^{-1} \circ r$ and $u_{2}: \mathcal{U}_{1} \mathcal{U}_{2} \rightarrow \mathcal{U}_{2}$ is defined by $u_{2}=\left.s\right|_{\mathcal{U}_{2}} ^{-1} \circ s$.

Given $g \in \mathcal{U}_{1} \mathcal{U}_{2}, g_{1}=u_{1}(g), g_{2}=u_{2}(g)$ are the only elements satisfying $g_{1} \in \mathcal{U}_{1}, g_{2} \in$ $\mathcal{U}_{2}, g=g_{1} g_{2}$. In fact, suppose there are $\left(h_{1}, h_{2}\right) \in \mathcal{U}_{1} \times \mathcal{U}_{2} \cap G^{(2)}$ such that $g=h_{1} h_{2}$. Then $r\left(h_{1}\right)=r(g)$. Since $\mathcal{U}_{1}$ is an open bisection, we have $h_{1}=\left.r\right|_{\mathcal{U}_{1}} ^{-1} \circ r(g)=g_{1}$. Analogously $h_{2}=g_{2}$.

Therefore, for every $g \in \mathcal{U}_{1} \mathcal{U}_{2}$,

$$
\left(f_{1} \cdot f_{2}\right)(g)=f_{1}\left(u_{1}(g)\right) f_{2}\left(u_{2}(g)\right)
$$

For $i=1,2, u_{i}: \mathcal{U}_{1} \mathcal{U}_{2} \rightarrow \mathcal{U}_{i}$ is continuous and $f_{i}: \mathcal{U}_{i} \rightarrow \mathbb{C}$ is continuous. Hence $f_{1} \cdot f_{2}$ is continuous on $\mathcal{U}_{1} \mathcal{U}_{2}$. Since $f_{1} \cdot f_{2}$ vanishes outside $\mathcal{U}_{1} \mathcal{U}_{2}$, we have $f_{1} \cdot f_{2} \in C_{c}\left(\mathcal{U}_{1} \mathcal{U}_{2}\right)$. 


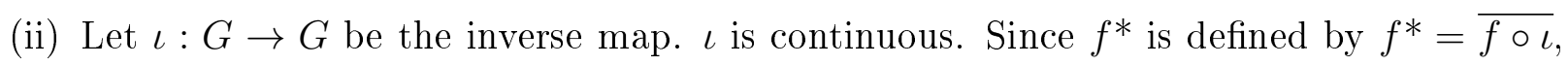
then $f^{*}$ is continuous.

Let $K=\operatorname{supp}(f)$ and $L=\operatorname{supp}\left(f^{*}\right)$. Then

$$
\begin{aligned}
L & =\overline{\left\{g \in G: f^{*}(g) \neq 0\right\}} \\
& =\overline{\left\{g \in G: \overline{f\left(g^{-1}\right)} \neq 0\right\}} \\
& =\overline{\left\{g \in G: f\left(g^{-1}\right) \neq 0\right\}} \\
& =\overline{\{g \in G: f(g) \neq 0\}} \\
& ={\overline{(\{g \in G: f(g) \neq 0\})^{-1}}}^{-1}, \quad \text { since the inversion is continuous, } \\
& =\overline{(\{g \in G: f(g) \neq 0\})}^{-1}, \\
& =K .
\end{aligned}
$$

The inversion on $G$ is continuous, thus $L$ is compact. Moreover, $L \subset \mathcal{U}^{-1}$. Thus $f^{*} \in C_{c}\left(\mathcal{U}^{-1}\right)$.

Theorem 3.3.9. Let $G$ be a locally compact Hausdorff second countable étale groupoid. $C_{c}(G)$ with the operations 3.2 is a $*$-algebra.

Proof. Clearly $C_{c}(G)$ is a vector space.

- The product is bilinear.

Let $f_{1}, f_{2}, f \in C_{c}(G), \lambda \in \mathbb{C}, g \in G$. Then,

$$
\begin{aligned}
{\left[\left(f_{1}+\lambda f_{2}\right) \cdot f\right](g) } & =\sum_{g_{1} g_{2}=g}\left(f_{1}+\lambda f_{2}\right)\left(g_{1}\right) f\left(g_{2}\right) \\
& =\sum_{g_{1} g_{2}=g} f_{1}\left(g_{1}\right) f\left(g_{2}\right)+\lambda \sum_{g_{1} g_{2}=g} f_{2}\left(g_{1}\right) f\left(g_{2}\right) \\
& =\left[f_{1} \cdot f\right](g)+\lambda\left[f_{2} \cdot f\right](g) .
\end{aligned}
$$


The proof for $f \cdot\left(f_{1}+\lambda f_{2}\right)$ is analogous.

- The product is associative.

Let $f_{1}, f_{2}, f_{3} \in C_{c}(G)$. Given $g \in G$,

$$
\begin{aligned}
{\left[f_{1} \cdot\left(f_{2} \cdot f_{3}\right)\right](g) } & =\sum_{g_{1} h=g} f_{1}\left(g_{1}\right)\left(f_{2} \cdot f_{3}\right)(h) \\
& =\sum_{g_{1} h=g} \sum_{g_{2} g_{3}=h} f_{1}\left(g_{1}\right) f_{2}\left(g_{2}\right) f_{3}\left(g_{3}\right) \\
& =\sum_{g_{1} g_{2} g_{3}=g} f_{1}\left(g_{1}\right) f_{2}\left(g_{2}\right) f_{3}\left(g_{3}\right) \\
& =\sum_{h g_{3}=g} \sum_{g_{1} g_{2}=h} f_{1}\left(g_{1}\right) f_{2}\left(g_{2}\right) f_{3}\left(g_{3}\right) \\
& =\sum_{h g_{3}=g}\left(\sum_{g_{1} g_{2}=h} f_{1}\left(g_{1}\right) f_{2}\left(g_{2}\right)\right) f_{3}\left(g_{3}\right) \\
& =\sum_{h g_{3}=g}\left(f_{1} \cdot f_{2}\right)(h) f_{3}\left(g_{3}\right) \\
& =\left[\left(f_{1} \cdot f_{2}\right) \cdot f_{3}\right](g) .
\end{aligned}
$$

- $f_{1} \cdot f_{2} \in C_{c}(G)$ if $f_{1}, f_{2} \in C_{c}(G)$.

Since the product is bilinear and, from Lemma 3.3.5 every function in $C_{c}(G)$ can be written as a finite sum of continuous functions supported on open bisections, it suffices to show that $f_{1} \cdot f_{2} \in C_{c}\left(\mathcal{U}_{1} \mathcal{U}_{2}\right)$ for $f_{1} \in C_{c}\left(\mathcal{U}_{1}\right), f_{2} \in C_{c}\left(\mathcal{U}_{2}\right)$ where $\mathcal{U}_{1}, \mathcal{U}_{2}$ are open bisections. Note that $\mathcal{U}_{1} \mathcal{U}_{2}$ is an open bisection by Lemma 3.3.6. However, we already proved $f_{1} \cdot f_{2} \in C_{c}\left(\mathcal{U}_{1} \mathcal{U}_{2}\right)$ in Lemma 3.3.8

- For $f \in C_{c}(G), f^{* *}=f$.

Let $g \in G$, then

$$
f^{* *}(g)=\overline{f^{*}\left(g^{-1}\right)}=\overline{\overline{f\left(\left(g^{-1}\right)^{-1}\right)}}=f(g) .
$$

- For $f_{1}, f_{2} \in C_{c}(G),\left(f_{1} \cdot f_{2}\right)^{*}=f_{2}^{*} \cdot f_{1}^{*}$. 
Let $g \in G$. Then,

$$
\begin{aligned}
\left(f_{1} \cdot f_{2}\right)^{*}(g) & =\overline{\left(f_{1} \cdot f_{2}\right)\left(g^{-1}\right)} \\
& =\sum_{g_{1} g_{2}=g^{-1}} \overline{f_{1}\left(g_{1}\right) f_{2}\left(g_{2}\right)} \\
& =\sum_{g_{1} g_{2}=g^{-1}} f_{2} *\left(g_{2}^{-1}\right) f_{1} *\left(g_{1}^{-1}\right) \\
& =\sum_{g_{2}^{-1} g_{1}^{-1}=g} f_{2}^{*}\left(g_{2}^{-1}\right) f_{1} *\left(g_{1}^{-1}\right), \\
& \text { making the change of variables } h_{1}=g_{2}^{-1}, h_{2}=g_{1}^{-1}, \\
& =\sum_{h_{1} h_{2}=g} f_{2} *\left(h_{1}\right) f_{1} *\left(h_{2}\right) \\
& =\left(f_{2} * \cdot f_{1} *\right)(g) .
\end{aligned}
$$

- The involution is conjugate-linear.

Let $f_{1}, f_{2} \in C_{c}(G), \lambda \in \mathbb{C}, g \in G$. Then,

$$
\left(f_{1}+\lambda f_{2}\right) *(g)=\overline{\left(f_{1}+\lambda f_{2}\right)\left(g^{-1}\right)}=\overline{f_{1}\left(g^{-1}\right)}+\overline{\lambda f_{2}\left(g^{-1}\right)}=f_{1} *(g)+\bar{\lambda} f_{2} *(g) .
$$

- If $f \in C_{c}(G)$, then $f^{*} \in C_{c}(G)$.

Since the involution is conjugate-linear, we can assume $f \in C_{c}(\mathcal{U})$ for an open bisection. It follows from Lemma 3.3 .8 that $f^{*} \in C_{c}(\mathcal{U})$.

In Theorem 3.3.9 we proved $C_{c}(G)$ is a $*$-algebra. Now we will equip this space with a norm such that its completion is a $\mathrm{C}^{*}$-algebra.

Lemma 3.3.10. Let $G$ be a locally compact Hausdorff second countable étale groupoid. $C_{c}\left(G^{(0)}\right)$ is a sub-*-algebra of $C_{c}(G)$. Moreover, $C_{c}\left(G^{(0)}\right)$ is commutative with product given by the pointwise multiplication and involution defined by $f^{*}(x)=\overline{f(x)}$. 
Proof. From Proposition 3.2 .12 it follows that $G^{(0)}$ is open. Then $C_{c}\left(G^{(0)}\right)$ is a subspace of $C_{c}(G)$ as described in Notation 3.3.2.

- $f^{*} \in C_{c}\left(G^{(0)}\right)$ if $f \in C_{c}\left(G^{(0)}\right)$

Let $f \in C_{c}\left(G^{(0)}\right)$. Then $f^{*} \in C_{c}(G)$ by Theorem 3.3.9. Let $g \in G$ such that $f(g) \neq 0$. Then $f^{*}(g)=\overline{f\left(g^{-1}\right)} \neq 0$. Thus $g^{-1} \in G^{(0)}$ by assumption. Then $g=g^{-1} \in G^{(0)}$. Therefore $f^{*}$ is also supported on $G^{(0)}$.

- $f_{1} \cdot f_{2}=f_{1} f_{2} \in C_{c}\left(G^{(0)}\right)$

Now let $f_{1}, f_{2} \in C_{c}\left(G^{(0)}\right)$. Then $f_{1} \cdot f_{2} \in C_{c}(G)$ by by Theorem 3.3.9. Given $g \in G$, we have

$$
\left(f_{1} \cdot f_{2}\right)(g)=\sum_{g_{1} g_{2}=g} f_{1}\left(g_{1}\right) f_{2}\left(g_{2}\right)
$$

Suppose there are $g_{1}, g_{2}$ such that $g=g_{1} g_{2}$ and $f_{1}\left(g_{1}\right) f_{2}\left(g_{2}\right) \neq 0$. Then $g_{1}, g_{2} \in G^{(0)}$. Hence $g \in G^{(0)}$ and $g=g_{1}=g_{2}$. Therefore, $\left(f_{1} \cdot f_{2}\right)(g)=f_{1}(g) f_{2}(g)$.

Therefore $C_{c}\left(G^{(0)}\right)$ is commutative.

Proposition 3.3.11. Let $G$ be a locally compact Hausdorff second countable étale groupoid. For each $f \in C_{c}(G)$, there is a constant $K_{f} \geq 0$ such that $\|\pi(f)\| \leq K_{f}$ for every *representation $\pi: C_{c}(G) \rightarrow B(H)$ of $C_{c}(G)$ on a Hilbert space $H$. If $f$ is supported on an open bisection, we can take $K_{f}=\|f\|_{\infty}$.

Proof. Suppose $\pi$ is a $*$-representation. Then $\left.\pi\right|_{C_{c}\left(G^{(0)}\right)}$ is a $*$-representation of the commutative $*$-algebra $C_{c}\left(G^{(0)}\right)$, and so $\|\pi(h)\| \leq\|h\|_{\infty}$ for every $h \in C_{c}\left(G^{(0)}\right)$.

Let $f \in C_{c}(G)$. There are $f_{1}, \ldots, f_{n}$ with $f=\sum_{i=1}^{n} f_{i}$ such that each $f_{i} \in C_{c}\left(\mathcal{U}_{i}\right)$ and $\mathcal{U}_{i}$ is an open bisection. Fix $i$, hence $f_{i}^{*} \in C_{c}\left(\mathcal{U}^{-1}\right)$ and therefore $f_{i}^{*} \cdot f_{i} \in C_{c}\left(\mathcal{U}^{-1} \mathcal{U}\right)$. However, 
$\mathcal{U U}^{-1}=s(\mathcal{U})$. In fact,

$$
\begin{aligned}
\mathcal{U}^{-1} \mathcal{U} & =\left\{g h: g \in \mathcal{U}^{-1}, h \in \mathcal{U}, s(g)=r(h)\right\} \\
& =\left\{g_{1}^{-1} g_{2}: g_{1}, g_{2} \in \mathcal{U}, r\left(g_{1}\right)=r\left(g_{2}\right)\right\} \\
& =\left\{g^{-1} g: g \in \mathcal{U}\right\} \quad \text { since } \mathcal{U} \text { is an open bisection } \\
& =s(\mathcal{U}) .
\end{aligned}
$$

Thus $f_{i}^{*} f_{i} \in C_{c}(s(\mathcal{U}))$. So

$$
\left\|\pi\left(f_{i}\right)\right\|^{2}=\left\|\pi\left(f_{i}^{*} \cdot f_{i}\right)\right\| \leq\left\|f_{i}^{*} \cdot f_{i}\right\|_{\infty}=\left\|f_{i}\right\|^{2} .
$$

Let $K_{f}=\sum_{i=1}^{n}\left\|f_{i}\right\|$. Applying triangle inequality, we have $\|\pi(f)\| \leq K_{f}$.

Proposition 3.3.12. Let $f \in C_{c}(G)$ such that $f \neq 0$. There exists a $*$-representation of $C_{c}(G)$ such that $\pi(f) \neq 0$.

Proof. Let $x \in G^{(0)}$ such that $f\left(h_{x}\right) \neq 0$ for some $h_{x} \in G_{x}$. Since $G_{x}$ is countable,

$$
\ell^{2}\left(G_{x}\right)=\left\{\left\{\xi_{g}\right\}_{g \in G_{x}}: \xi_{g} \in \mathbb{C}, \sum_{g \in G_{x}}\left|\xi_{g}\right|^{2}<\infty\right\}
$$

is a Hilbert space with inner product given by

$$
\langle\xi, \zeta\rangle=\sum_{g \in G_{x}} \xi_{g} \overline{\zeta_{g}}
$$

Define $\pi_{x}: C_{c}(G) \rightarrow B\left(\ell^{2}\left(G_{x}\right)\right)$ by

$$
\left(\pi_{x}\left(f_{1}\right) \xi\right)_{g}=\sum_{h_{1} h_{2}=g} f_{1}\left(h_{1}\right) \xi_{h_{2}} .
$$


Note that

$$
\left(\pi_{x}\left(f_{1}\right) \xi\right)_{g}=\sum_{h \in G_{s(g)}} f_{1}\left(g h^{-1}\right) \xi_{h}=\sum_{h \in G^{r}(g)} f_{1}(h) \xi_{h^{-1} g}
$$

making the change of variables $h_{2}=h, h_{1}=g h^{-1}$ in the first sum, and $h_{1}=h, h_{2}=h^{-1} g$ in the second sum. Note that $\pi_{x}$ is linear.

- $\pi_{x}$ is well-defined.

We will prove that the image of $\pi_{x}$ is in $B\left(\ell^{2}\left(G_{x}\right)\right)$. Since $\pi_{x}$ is linear, it is sufficient to show that $\pi_{x}\left(f_{1}\right) \in B\left(\ell^{2}\left(G_{x}\right)\right)$ for every $f \in C_{c}(\mathcal{U})$ such that $\mathcal{U} \subset G$ is an open bisection.

Let $\mathcal{U} \subset G$ be an open bisection, $f_{1} \in C_{c}(\mathcal{U})$. Let $L$ denote the set of $g \in G_{x}$ such that there exists $h \in G^{r(g)}$ satisfying $f(h) \neq 0$. $h$ is unique for every $g \in L$ and it will be denoted by $h^{r(g)}$. Then, for every $\xi \in \ell^{2}\left(G_{x}\right), g \in L$,

$$
\left(\pi_{x}\left(f_{1}\right) \xi\right)_{g}=f_{1}\left(h^{r(g)}\right) \xi_{\left(h^{r(g)}\right)^{-1} g}
$$

Note that $\left(\pi_{x}\left(f_{1}\right) \xi\right)_{g}=0$ if $g \in G_{x} \backslash L$.

Suppose there are $g_{1}, g_{2} \in L$ such that $\left(h^{r\left(g_{1}\right)}\right)^{-1} g_{1}=\left(h^{r\left(g_{2}\right)}\right)^{-1} g_{2}$. Then $s\left(h^{r\left(g_{1}\right)}\right)=$ $s\left(h^{r\left(g_{2}\right)}\right)$. Since $h^{r\left(g_{1}\right)}, h^{r\left(g_{2}\right)} \in \mathcal{U}$, we have $h^{r\left(g_{1}\right)}=h^{r\left(g_{2}\right)}$. Then $g_{1}=g_{2}$. Since $h^{r(g)} g \in$ $G_{x}$, the family $\left\{\xi_{h^{r(g)} g}\right\}_{g \in L}$ has distinct elements. Hence,

$$
\begin{aligned}
\left\|\pi_{x}\left(f_{1}\right) \xi\right\|^{2} & =\sum_{g \in G_{x}}\left|\left(\pi_{x}\left(f_{1}\right) \xi\right)_{g}\right|^{2} \\
& =\sum_{g \in L}\left|\left(\pi_{x}\left(f_{1}\right) \xi\right)_{g}\right|^{2} \\
& =\sum_{g \in L}\left|f_{1}\left(h^{r(g)}\right) \xi_{\left(h^{r(g)}\right)^{-1} g}\right|^{2} \\
& \leq\left\|f_{1}\right\|_{\infty}^{2} \sum_{g \in L}\left|\xi_{\left(h^{r(g)}\right)^{-1} g}\right|^{2}
\end{aligned}
$$




$$
\begin{aligned}
& \leq\left\|f_{1}\right\|_{\infty}^{2} \sum_{h \in G_{x}}\left|\xi_{h}\right|^{2}, \text { since }\left\{\xi_{h^{r(g)} g}\right\}_{g \in L} \text { has distinct elements, } \\
& \leq\left\|f_{1}\right\|_{\infty}^{2}\|\xi\|^{2}
\end{aligned}
$$

Therefore $\left\|\pi_{x}\left(f_{1}\right)\right\| \leq\left\|f_{1}\right\|_{\infty}<\infty$.

- $\pi_{x}(f) \neq 0$.

Let $\xi \in \ell^{2}\left(G_{x}\right)$ such that $\xi_{x}=1$ and $\xi_{l}=0$ if $l \neq x$. Let $g \in G_{x}$ such that $f(g) \neq 0$.

Then, by definition of $\xi$,

$$
\left(\pi_{x}(f) \xi\right)_{g}=\sum_{h \in G^{r(g)}} f(h) \xi_{h^{-1} g}=f(g) \xi_{g^{-1} g}=f(g) \xi_{x}=f(g) \neq 0 .
$$

- $\pi_{x}\left(f_{1} \cdot f_{2}\right)=\pi_{x}\left(f_{1}\right) \pi_{x}\left(f_{2}\right)$

Let $f_{1}, f_{2} \in C_{c}(G)$. Then,

$$
\begin{aligned}
{\left[\pi_{x}\left(f_{1}\right)\left(\pi_{x}\left(f_{2}\right) \xi\right)\right]_{g} } & =\sum_{g_{1} h=g} f_{1}\left(g_{1}\right)\left(\pi_{x}\left(f_{2}\right) \xi\right)_{h} \\
& =\sum_{g_{1} h=g} f_{1}\left(g_{1}\right) \sum_{g_{2} g_{3}=h} f_{2}\left(g_{2}\right) \xi_{g_{3}} \\
& =\sum_{g_{1} g_{2} g_{3}=g} f_{1}\left(g_{1}\right) f_{2}\left(g_{2}\right) \xi_{g_{3}} \\
& =\sum_{h g_{3}=g}\left(\sum_{g_{1} g_{2}=h} f_{1}\left(g_{1}\right) f_{2}\left(g_{2}\right)\right) \xi_{g_{3}} \\
& =\sum_{h g_{3}=g}\left(f_{1} \cdot f_{2}\right)(h) \xi_{g_{3}} \\
& =\left[\pi_{x}\left(f_{1} \cdot f_{2}\right) \xi\right]_{g} .
\end{aligned}
$$

- $\pi_{x}\left(f_{1}^{*}\right)=\pi_{x}\left(f_{1}\right)^{*}$ 
Let $f_{1} \in C_{c}(G), \xi \in \ell^{2}\left(G_{x}\right)$. Then

$$
\begin{aligned}
\left\langle\xi, \pi_{x}\left(f_{1} *\right) \xi\right\rangle & =\sum_{g \in G_{x}} \xi_{g} \overline{\left[\pi_{x}\left(f_{1}^{*}\right)\right]_{g}} \\
& =\sum_{g \in G_{x}} \xi_{g} \sum_{h \in G_{x}} \overline{f_{1}^{*}\left(g h^{-1}\right) \xi_{h}} \\
& =\sum_{g \in G_{x}} \xi_{g} \sum_{h \in G_{x}} f_{1}\left(h g^{-1}\right) \overline{\xi_{h}} \\
& =\sum_{h \in G_{x}}\left(\sum_{g \in G_{x}} f_{1}\left(h g^{-1}\right) \xi_{g}\right) \overline{\xi_{h}} \\
& =\sum_{h \in G_{x}}\left[\pi_{x}\left(f_{1}\right) \xi\right]_{h} \overline{\xi_{h}} \\
& =\left\langle\pi_{x}\left(f_{1}\right) \xi, \xi\right\rangle .
\end{aligned}
$$

Therefore $\pi_{x}\left(f_{1}^{*}\right)=\pi_{x}\left(f_{1}\right)^{*}$.

It follows that $\pi_{x}$ is a $*$-representation of $C_{c}(G)$ such that $\pi_{x}(f) \neq 0$.

Theorem 3.3.13. Assume $G$ is a locally compact Hausdorff second countable étale groupoid. There exists a $\mathrm{C}^{*}$-algebra $C^{*}(G)$ such that $C_{c}(G)$ is dense in $C^{*}(G)$ and the norm on $C^{*}(G)$ satisfies

$$
\|f\|=\sup \left\{\|\pi(f)\|: \pi: C_{c}(G) \rightarrow B\left(H_{\pi}\right) \text { is a } * \text {-representation of } C_{c}(G)\right\},
$$

for every $f \in C_{c}(G)$.

Proof. For every $f \in C_{c}(G)$, Proposition 3.3 .11 shows that the set

$$
\left\{\|\pi(f)\|: \pi \text { is a } * \text {-representation of } C_{c}(G)\right\}
$$

is bounded above, and it is nonempty because of the zero representation. So we can define 
$\rho: C_{c}(G) \rightarrow[0, \infty)$ by

$$
\rho(f)=\sup \left\{\|\pi(f)\|: \pi: C_{c}(G) \rightarrow B\left(H_{\pi}\right) \text { is a } * \text {-representation }\right\} .
$$

$\rho$ is a norm on $C_{c}(G)$. In fact, given $\lambda \in \mathbb{C}, f \in C_{c}(G)$,

$$
\rho(\lambda f)=\sup _{\pi}\|\pi(\lambda f)\|=|\lambda| \sup _{\pi}\|\pi(f)\|=|\lambda| \rho(f) .
$$

Given $f_{1}, f_{2} \in C_{c}(G)$,

$$
\rho\left(f_{1}+f_{2}\right)=\sup _{\pi}\left\|\pi\left(f_{1}+f_{2}\right)\right\| \leq \sup _{\pi}\left\|\pi\left(f_{1}\right)\right\|+\sup _{\pi}\left\|\pi\left(f_{2}\right)\right\|=\rho\left(f_{1}\right)+\rho\left(f_{2}\right) .
$$

Given $f \in C_{c}(G)$ such that $f \neq 0, \rho(f)>0$ by Proposition 3.3.12.

The norm is submultiplicative. Given $f_{1}, f_{2} \in C_{c}(G)$,

$$
\rho\left(f_{1} \cdot f_{2}\right)=\sup _{\pi}\left\|\pi\left(f_{1} \cdot f_{2}\right)\right\|=\sup _{\pi}\left\|\pi\left(f_{1}\right) \pi\left(f_{2}\right)\right\| \leq \sup _{\pi}\left\|\pi\left(f_{1}\right)\right\| \sup _{\pi}\left\|\pi\left(f_{2}\right)\right\|=\rho\left(f_{1}\right) \rho\left(f_{2}\right) .
$$

Given $f \in C_{c}(G)$,

$$
\rho\left(f^{*}\right)=\sup _{\pi}\left\|\pi\left(f^{*}\right)\right\|=\sup _{\pi}\left\|\pi(f)^{*}\right\|=\sup _{\pi}\|\pi(f)\|=\rho(f) .
$$

Moreover, $\rho$ satisfies the $C^{*}$-identity. Indeed, given $f \in C_{c}(G)$,

$$
\rho\left(f^{*} f\right)=\sup _{\pi}\left\|\pi\left(f^{*} f\right)\right\|=\sup _{\pi}\left\|\pi(f)^{*} \pi(f)\right\|=\sup _{\pi}\|\pi(f)\|^{2}=\sup _{\pi}\|\pi(f)\|=\rho(f)^{2} .
$$

So we define $C^{*}(G)$ to be the completion of $C_{c}(G)$ with respect to the norm $\rho$. $C^{*}(G)$ is a $\mathrm{C}^{*}$-algebra.

Definition 3.3.14. Given a locally compact Hausdorff second countable étale groupoid $G$, $C^{*}(G)$ is called the full $C^{*}$-algebra of $G$.

Remark 3.3.15. In this thesis we also say $\mathrm{C}^{*}(\mathrm{G})$ is the groupoid $C^{*}$-algebra for $G$. However, 
this is not the unique $\mathrm{C}^{*}$-algebra defined as the completion of $C_{c}(G)$. For example, in [9] the reduced $\mathrm{C}^{*}$-algebra is defined as the closure of $C_{c}(G)$ with respect to the norm $\|f\|=\left\|\pi_{\lambda}(f)\right\|$, where $\pi_{\lambda}$ is a $*$-representation of $C_{c}(G)$ called the regular representation of $C_{c}(G)$.

Lemma 3.3.16. Let $G$ be a locally compact second countable Hausdorff étale groupoid. Then $C_{0}\left(G^{(0)}\right)$ is a sub-C*-algebra of $C^{*}(G)$ and the norm on $C_{0}\left(G^{(0)}\right)$ is the uniform norm. Moreover, $C_{c}\left(G^{(0)}\right)$ is dense in $C_{0}\left(G^{(0)}\right)$.

Proof. From Proposition 3.2 .12 , we have that $G^{(0)}$ is clopen in $G$. Moreover, $G^{(0)}$ is an open bisection because $r$ and $s$ are injective on $G^{(0)}$. From Lemma 3.3 .10 we have that $C_{c}\left(G^{(0)}\right)$ is a sub-*-algebra with product given by pointwise multiplication and involution defined by $f^{*}(x)=\overline{f(x)}$.

Let $h \in C_{c}\left(G^{(0)}\right)$. It follows from Proposition 3.3.11 that $\|\pi(h)\| \leq\|h\|_{\infty}$ for every representation $\pi$ of $C_{c}(G)$. Then $\|h\| \leq\|h\|_{\infty}$.

We will show that $\|h\|=\|h\|_{\infty}$ for every $h \in C_{c}\left(G^{(0)}\right)$. Given $x \in G^{(0)}$, let $\pi_{x}: C_{c}(G) \rightarrow$ $B\left(\ell^{2}\left(G_{x}\right)\right)$ be the *-representation as in the proof of Proposition 3.3.12. Then, for every $h \in C_{c}\left(G^{(0)}\right), \xi \in \ell^{2}\left(G_{x}\right)$, we have

$$
\left[\pi_{x}(h) \xi\right]_{g}=\sum_{g_{1} g_{2}=g} h\left(g_{1}\right) \xi_{g_{2}}=h(r(g)) \xi_{g}
$$

because $h$ vanishes outside $G^{(0)}$. Let $\zeta \in \ell^{2}\left(G_{x}\right)$ such that $\zeta_{g}=0$ if $g \neq x$ and $\zeta_{x}=1$. Then $\|\zeta\|=1$ and

$$
\left\|\pi_{x}(h)\right\|^{2} \geq\left\|\pi_{x}(h) \zeta\right\|^{2}=\sum_{g \in G_{x}}\left|\pi_{x}(h) \zeta_{g}\right|^{2}=\left|\pi_{x}(h)\right|^{2}=|h(r(x))|^{2}=|h(x)|^{2} .
$$

Then,

$$
\|h\|=\sup \left\{\|\pi(h)\|: \pi: C_{c}(G) \rightarrow B\left(\mathcal{H}_{\pi}\right) \text { is a } * \text {-representation }\right\}
$$




$$
\geq \sup _{x \in G^{(0)}}\left|\pi_{x}(h)\right|=\sup _{x \in G^{(0)}}|h(x)|=\|h\|_{\infty}
$$

Then $\|h\|=\|h\|_{\infty}$. Recall that $C_{0}\left(G^{(0)}\right)$ is the closure of $C_{c}\left(G^{(0)}\right)$ with respect to the norm $\|\cdot\|_{\infty}$. Therefore, $C_{0}\left(G^{(0)}\right)$ is a sub-C ${ }^{*}$-algebra of $C^{*}(G)$.

Example 3.3.17. Let $X$ be the groupoid of Example 3.2.5 and assume that $X$ is locally compact Hausdorff second countable. Then the operations on $C_{c}(X)$ are the pointwise multiplication and the complex conjugate by Lemma 3.3.10. It follows from Lemma 3.3.16 that $C_{0}(X)=C^{*}(X)$ and the norm on this $\mathrm{C}^{*}$-algebra is the uniform norm.

Now we define the inductive limit topology. Later we show that convergence with respect to the inductive limit topology on $C_{c}(G)$ implies convergence in the norm of $C^{*}(G)$. This definition can be found in [10]. Then we will prove that $C^{*}(G)$ is separable.

Definition 3.3.18. Suppose $X$ is a locally compact Hausdorff second countable space. Given a sequence $\left\{f_{n}\right\}_{n \in \mathbb{N}}$ on $C_{c}(X)$ and $f \in C_{c}(X)$, we say that $f_{n} \rightarrow f$ with respect to the inductive limit topology if, and only if, $f_{n} \rightarrow f$ uniformly and there exists a compact set $K$ in $X$ such that, eventually, all the $f_{n}$ and $f$ vanish outside $K$. Given a topological space $Y$, we will say that a function $F: C_{c}(X) \rightarrow Y$ is continuous in the inductive limit topology if $F\left(f_{n}\right) \rightarrow F(f)$ whenever $f_{n} \rightarrow f$ with respect to the inductive limit topology.

Lemma 3.3.19. Let $G$ be a locally compact Hausdorff second countable Hausdorff étale groupoid. Let $\left\{f_{i}\right\}_{i \in \mathbb{N}}$ be a sequence in $C_{c}(G)$ such that $f_{i} \rightarrow f$ with respect to the inductive limit topology. Then $f_{i} \rightarrow f$ in $C^{*}(G)$.

Proof. Let $K$ be a compact set such that $f_{i}$ eventually vanishes outside $K$. Let $\mathcal{U}_{1}, \ldots, \mathcal{U}_{n}$ be open bisections which cover $K$. Let $p_{1}, \ldots, p_{n}$ be a partition of unit subordinate to the open cover.

Let $\pi: C_{c}(G) \rightarrow B\left(H_{\pi}\right)$ be a $*$-representation of $C_{c}(G)$. Fix $j=1, \ldots, n$. Then $p_{j} f$ and each $p_{j} f_{i}$ are supported on the open bisection $\mathcal{U}_{j}$. Hence, by Proposition 3.3.11, we have for 
every $i$,

$$
\left\|\pi\left(p_{j} f_{i}\right)-\pi\left(p_{j} f\right)\right\| \leq\left\|p_{j} f_{i}-p_{j} f\right\|_{\infty} \leq\left\|f_{i}-f\right\|_{\infty} .
$$

By taking the supremum on $\pi$, we have $\left\|p_{j} f_{i}-p_{j} f\right\| \leq\left\|f_{i}-f\right\|_{\infty}$. Since $f_{i}$ converges to $f$ uniformly, it follows that $p_{j} f_{i} \rightarrow p_{j} f$ in $C^{*}(G)$. Since $\left\{p_{j}\right\}$ is a partition of unit subordinate to the open cover of $K$, and the sequence $f_{i}$ is eventually supported on $K$, it follows that $f_{i} \rightarrow f$ in $C^{*}(G)$.

We state the Stone-Weierstrass theorem below, which can be found in [11]. This theorem will be used to prove that $C^{*}(G)$ is separable.

Theorem 3.3.20. (Stone-Weierstrass theorem for complex-valued functions) Let $K$ be a compact space, $A$ a subalgebra of $C(X)$ which separates points in $K$, that is, for every $x_{1}, x_{2} \in K$, there is $f \in A$ such that $f\left(x_{1}\right) \neq f\left(x_{2}\right)$. Assume $\bar{f} \in A$ for every $f \in A$. Moreover, suppose that for every $x \in K$ there exists $f \in A$ with $f(x) \neq 0$. Therefore $A$ is dense in $C(K)$.

Lemma 3.3.21. Let $X$ be a locally compact Hausdorff second countable space. Let $U$ be an open subset of $X$ with compact closure. Then $C_{c}(U)$ is separable with respect to the supremum norm.

Proof. Let $\mathcal{F}=\left\{U_{n}\right\}$ be a countable family of open sets such that $\overline{U_{n}}$ is compact and $U=\cup_{n} \overline{U_{n}}$. Given $n, m$ such that $\overline{U_{n}} \cap \overline{U_{m}}=\varnothing$, let $f_{n, m} \in C_{c}(U)$ such that $\left.f_{n, m}\right|_{\overline{U_{n}}}=1$ and $\left.f_{n, m}\right|_{\overline{U_{m}}}=0$.

Let $A$ be the algebra generated by $f_{n, m}$, and $A_{0}$ be the set generated by sums and products of $f_{n, m}$, and also by multiplication of scalars in $\mathbb{Q}+i \mathbb{Q}$. Note that $A_{0}$ is countable and dense in $A$. Moreover, if $f \in A$, then $\bar{f} \in A$.

Note that $A$ separates points in $U$. Let $x_{1}, x_{2} \in U$. It follows from Propositions 2.4.1 and 2.4 .2 that there are $U_{1}, U_{2} \in \mathcal{F}$ such that $\overline{U_{1}} \cap \overline{U_{2}}=\varnothing, x_{1} \in U_{1}, x_{2} \in U_{2}$. Then $f_{1,2}\left(x_{1}\right)=1$ and $f_{1,2}\left(x_{2}\right)=0$. 
Therefore, by the Stone-Weierstrass theorem, $A_{0}$ is dense in $C_{c}(U)$.

Proposition 3.3.22. Let $G$ be a locally compact Hausdorff second countable étale groupoid. Then $C^{*}(G)$ is separable.

Proof. Let $I$ be a countable family of open bisections with compact support that covers $G$. From Lemma 3.3.21, there exists a countable subset $A_{\mathcal{U}}$ of $C_{c}(\mathcal{U})$ such that $A_{\mathcal{U}}$ is dense in $C_{c}(\mathcal{U})$ with respect to the supremum norm. Let $A_{0}$ be the set generated by finite sums of elements in $\cup_{\mathcal{U} \in I} A_{\mathcal{U}}$. Then $A_{0}$ is countable.

Let $f \in C_{c}(G)$. There is a finite family $\mathcal{U}_{1}, \ldots, \mathcal{U}_{n} \in I$ that covers the support of $f$. Let $p_{1}, \ldots, p_{n}$ be a partition of unit subordinate to $\mathcal{U}_{1}, \ldots, \mathcal{U}_{n}$.

Let $\varepsilon>0$. Given $i=1, \ldots, n, p_{i} f \in C_{c}\left(\mathcal{U}_{i}\right)$. Then there exists $F_{i} \in A_{\mathcal{U}_{i}}$ satisfying $\left\|F_{i}-p_{i} f_{i}\right\|_{\infty}<\varepsilon / n$. It follows from Proposition 3.3.11 that $\left\|\pi\left(F_{i}\right)-\pi\left(p_{i} f\right)\right\|<\varepsilon / n$, for every *-representation of $C_{c}(G)$. Then $\left\|F_{i}-p_{i} f\right\|<\varepsilon / n$.

Let $F=\sum_{i=1}^{n} F_{i}$. Then $F \in A_{0}$. Moreover,

$$
\|F-f\|=\left\|\sum_{i=1}^{n} F_{i}-\sum_{i=1}^{n} p_{i} f_{i}\right\| \leq \sum_{i=1}^{n}\left\|F_{i}-p_{i} f_{i}\right\|<n \frac{\varepsilon}{n}=\varepsilon .
$$

Therefore $A_{0}$ is dense in $C_{c}(G)$. Since $C_{c}(G)$ is dense in $C^{*}(G)$, it follows that $C^{*}(G)$ is separable. 


\section{Chapter 4}

\section{Renault's Disintegration Theorem}

Neshveyev's theorems describe KMS states $\varphi$ on a groupoid $\mathrm{C}^{*}$-algebra. It is possible to write $\varphi(f)$ as an integral on $G^{(0)}$ with respect to a probability measure $\mu$ such that, for each $x$, there is a state $\varphi_{x}$ on $C^{*}\left(G_{x}^{x}\right)$. Moreover, the family of states $\varphi_{x}$ depends on $\mu$.

Recall from the theory of $\mathrm{C}^{*}$-algebras [2], [16] that we can write $\varphi(f)=\langle\pi(f) \xi, \xi\rangle$ for a representation $\pi: C^{*}(G) \rightarrow \mathcal{H}$, and $\xi \in \mathcal{H}$. Analogously, we can write $\varphi_{x}(f)=\left\langle\pi_{x}(f) \xi_{x}, \xi_{x}\right\rangle$ for a representation $\pi: C^{*}\left(G_{x}^{x}\right) \rightarrow \mathcal{H}_{x}$, and $\xi_{x} \in \mathcal{H}$

A fundamental step in the proof of Neshveyev's theorem is the Renault's disintegration theorem which shows a relation between $\pi$ and the family of $\pi_{x}$. Moreover, it proves that we can assume $\xi=\left\{\xi_{x}\right\}_{x \in G^{(0)}}$. In this case we say $\xi \in \int_{G^{(0)}}^{\oplus} \mathcal{H}_{x} d \mu(x)$.

The results in this chapter can be found in [4] and [10].

\subsection{Haar Systems}

Given a measure $\mu$ on $G^{(0)}$, it is possible to define a measure $\nu$ on $G$ if we have a family of measures $\lambda^{x}$ supported on $G^{x}$. The family $\left\{\lambda^{x}\right\}_{x \in G^{(0)}}$ is called a Haar system. A Haar system is a generalization of the notion of Haar measures on groups.

If we fix the Haar system, we can define a family of measures $\lambda_{x}$ supported on $G_{x}$ and, 
with this family, we can construct another measure $\nu^{-1}$ on $G$. When $\nu, \nu^{-1}$ are equivalent, we say $\mu$ is quasi-invariant.

Given a state $\varphi$ on $C^{*}(G)$, it follows from Neshveyev's first theorem that there is corresponding probability measure $\mu$ on $G^{(0)}$. In addition, if this state is KMS, then this measure is necessarily quasi-invariant with respect to the Haar system given by counting measures $\lambda^{x}$ on $G^{x}$.

The results in this section are based on [4] and [10].

Definition 4.1.1. Let $G$ be a locally compact Hausdorff groupoid, a (left) Haar system $\left\{\lambda^{x}\right\}_{x \in G^{(0)}}$ for $G$ is a family of Radon measures on $G$, such that the following conditions hold:

(i) $\operatorname{supp}\left(\lambda^{x}\right)=G^{x}$ for every $x \in G^{(0)}$;

(ii) (continuously varying) for $f \in C_{c}(G)$, the function

$$
x \mapsto \int_{G} f(g) d \lambda^{x}(g)
$$

is in $C_{c}\left(G^{(0)}\right)$;

(iii) (left invariance) for $f \in C_{c}(G), h \in G$,

$$
\int_{G} f(h g) d \lambda^{s(h)}(g)=\int_{G} f(g) d \lambda^{r(h)}(g) .
$$

Now we prove that Haar systems are a generalization of Haar measures, as defined in [6]. A topological group is a group $G$ endowed with a topology such that its operations are continuous functions. Given a locally compact Hausdorff group G, a (left) Haar measure is a non-zero Radon Borel measure $\mu$ on $G$ satisfying

$$
\mu(g A)=\mu(A) \quad \text { for every } g \in G \text { and } A \subset G \text { measurable. }
$$


Lemma 4.1.2. Let $G$ be a locally compact Hausdorff topological group. Let $\mu$ be a Radon measure on $G$. Then $\mu$ is a Haar measure if, and only if, $\left\{\lambda^{x}\right\}_{x \in G^{(0)}}$ is a Haar system assuming $\lambda^{1}=\mu$.

Proof. Note that a group $G$ is a groupoid such that $G^{(0)}=\{1\}$.

Assume $\mu$ is a Haar measure. First we show that $\operatorname{supp}(\mu)=G^{1}=G$. We prove that the properties of Haar system hold for $\left\{\lambda^{x}\right\}_{x \in G^{(0)}}$

(i) $\operatorname{supp}\left(\lambda^{1}\right)=G^{1}=G$

Suppose there exists an open non-empty set $U \subset G$ such that $\mu(U)=0$. Let $h \in U$. Then $h^{-1} U$ is an open neighborhood of 1 and $\mu\left(h^{-1} U\right)=\mu(U)=0$. Let $V=h^{-1} U$.

Let $K$ be a compact set. For every $g \in K, g V$ is an open neighborhood of $g$. Then there are $g_{1}, \ldots, g_{n} \in K$ such that $g_{1} V, \ldots, g_{n} V$ is an open cover of $K$. However, $\mu\left(g_{1} V\right)=0$ for every $i=1, \ldots, n$. Hence $\mu(K)=0$. Then, by definition of Radon measure, we have

$$
\mu(G)=\sup \{K \subset G: K \text { is compact }\}=0,
$$

which leads to a contradiction. Therefore, $\mu$ is positive on all open subsets of $G$.

Now let $F$ be the support of $\mu$. Then, by definition, $F$ is closed and $\mu(G \backslash F)=0$. Since $G \backslash F$ is open, we have $G \backslash F=\varnothing$, that is, $G=F$.

(ii) Property (ii) in Definition 4.1.1 since $G^{(0)}$ is singleton.

(iii) Left invariance in Definition 4.1.1

Let $f \in C_{c}(G)$. We can assume $f \geq 0$ without loss of generality. Let $A \subset G$ measurable and $a \geq 0$ such that $\varphi=a \chi_{A} \leq f$.

Given $h \in G$, the function $g \mapsto \varphi(h g)$ satisfies $\varphi(h g)=\chi_{h^{-1} A}(g) \leq f(h g)$ for every 
$g \in G$. Hence,

$$
\int_{G} \varphi(g) d \mu(g)=a \mu(A)=a \mu\left(h^{-1} A\right)=\int_{G} \varphi(h g) d \mu(g) .
$$

Equation 4.1 holds for every simple function $\varphi \leq f$ by linearity of the integral. Then, by taking the supremum over $\varphi \leq f$,

$$
\int_{G} f(g) d \mu(g) \leq \int_{G} f(h g) d \mu(g)
$$

The other inequality is proven analogously.

Conversely, assume $\left\{\lambda^{x}\right\}_{x \in G^{(0)}}$ is a Haar system with $\lambda^{1}=\mu$.

Let $U \subset G$ be an open set and let $h \in G$. Note that there is a correspondence between measurable functions $0 \leq f \leq \chi_{U}$ and $0 \leq \tilde{f} \leq \chi_{h^{-1} U}$ given by the relation

$$
\widetilde{f}(g)=f\left(h^{-1} g\right)
$$

In fact, given $f$ satisfying $f \leq \chi_{U}, g \in G$ such that $\widetilde{f}(g) \neq 0$, then $\tilde{f}\left(h^{-1} g\right) \neq 0$. Hence $h^{-1} g \in U$. Then $g \in h U$. Therefore $0 \leq \tilde{f} \leq \chi_{h U}$. Analogously, given $0 \leq \tilde{f} \leq \chi_{h U}$, there is a unique $0 \leq f \leq \chi_{U}$ such that 4.2 holds.

Hence, by Lemma 2.4.14.

$$
\mu(U)=\sup _{\substack{f \in C_{c}(G) \\ 0 \leq f \leq \chi_{U}}} \int_{G} f(g) d \mu(g)=\sup _{\substack{f \in C_{c}(G) \\ 0 \leq f \leq \chi_{U}}} \int_{G} f\left(h^{-1} g\right) d \mu(g)=\sup _{\substack{\tilde{f} \in C_{c}(G) \\ 0 \leq \widetilde{f} \leq \chi_{U}}} \int_{G} \widetilde{f}(g) d \mu(g)=\mu(h U) .
$$

Let $A$ be a Borel set. Since the map $g \mapsto h g$ is a homeomorphism, for every open set $V \supset h A$, there exists a unique open set $A \subset$ such that $V=h U$. Then,

$$
\mu(A)=\inf _{\substack{A \subset U \\ U \text { open }}} \mu(U)=\inf _{\substack{A \subset U \\ U \text { open }}} \mu(h U)=\inf _{\substack{h A \subset V \\ V \text { open }}} \mu(V)=\mu(h A)
$$


Therefore $\mu$ is a Haar measure.

Now we prove that the family $\lambda^{x}$ of counting measures on $G^{x}$ is a Haar system. In fact, this will be the Haar system used in Chapter 5 .

Proposition 4.1.3. Let $G$ be a locally compact étale groupoid. If $\lambda^{x}$ is the counting measure on $G^{x}$, then $\left\{\lambda^{x}\right\}_{x \in G^{(0)}}$ is a left Haar system.

Proof. Each $G^{x}$ is countable, then the counting measure $\lambda^{x}$ is well defined. We show the properties of Definition 4.1.1 hold.

(i) Given $x \in G^{(0)}, G^{x}$ is closed in $G$. Moreover, by Proposition 3.2.11, $G^{x}$ is countable. Then $G^{x}$ is the support of $\lambda^{x}$.

Since $G$ is étale, for every $g \in G^{x}$ there exists an open bisection $\mathcal{U}_{g}$ such that $\mathcal{U}_{g} \cap G^{x}=$ $\{g\}$. Let $K \subset G$ be compact. Then $K^{\prime}=K \cap G^{x}$ is compact and $\lambda^{x}(K)=\lambda^{x}\left(K^{\prime}\right)$. Moreover, there are $g_{1}, \ldots g_{n} \in G^{x}$ such that $K^{\prime} \subset \mathcal{U}_{g_{1}} \cup \ldots \cup \mathcal{U}_{g_{n}}$. Hence,

$$
\lambda^{x}(K)=\lambda^{x}\left(K^{\prime}\right) \leq \lambda^{x}\left(\mathcal{U}_{g_{1}} \cup \ldots \cup \mathcal{U}_{g_{n}}\right) \leq \sum_{i=1}^{n} \lambda^{x}\left(\mathcal{U}_{g_{i}}\right)=\sum_{i=1}^{n} \lambda^{x}\left(g_{i}\right)=n
$$

Then $\lambda^{x}$ is finite on compact subsets. Therefore, $\lambda^{x}$ is Radon by Proposition 2.4.10.

(ii) Let $f \in C_{c}(G)$. First assume $f \in C_{c}(\mathcal{U})$, where $\mathcal{U}$ is an open bisection. Let $K=$ $\operatorname{supp}(f)$. Define the open set $V=r(\mathcal{U})$ and the compact set $L=r(K)$. Hence, we can define the function $\tilde{f} \in C_{c}(r(V))$ by

$$
\tilde{f}(y)= \begin{cases}f\left(\left.r\right|_{\mathcal{U}} ^{-1}(y)\right), & \text { if } y \in L \\ 0, & \text { otherwise. }\end{cases}
$$

Note that, for every $y \in G^{(0)}$,

$$
\tilde{f}(y)=\int_{G} f(g) d \lambda^{y}(g)
$$


Now let $f \in C_{c}(G)$ arbitrary with support $K=\operatorname{supp}(f)$. Since $K$ is compact and $G$ is étale, $K$ has a finite open cover $\mathcal{U}_{1}, \ldots, \mathcal{U}_{n}$ of open bisections. By Proposition 3.3.5, there are $f_{1}, \ldots, f_{n}$ such that $f=f_{1}+\ldots+f_{n}$ and each $f_{i} \in C_{c}\left(\mathcal{U}_{i}\right)$. As we have shown, for every $i=1, \ldots, n$, the function

$$
y \mapsto \int_{G} f_{i}(g) d \lambda^{y}(g)
$$

is continuous and compactly supported. Then the function

$$
y \mapsto \int_{G} f(g) d \lambda^{y}(g)
$$

is continuous and compactly supported.

(iii) Given $f \in C_{c}(G), h \in G$,

$$
\int_{G} f(h g) d \lambda^{s(h)}(g)=\sum_{g \in G^{s(h)}} f(h g)
$$

We can use the change of variables $\tilde{g}=h g$ because the function from $G^{s(h)}$ to $G^{r(h)}$ defined by $g \mapsto h g$ is injective. Then we have $\tilde{g} \in G^{r(h)}$ and

$$
\sum_{g \in G^{s(h)}} f(h g)=\sum_{\tilde{g} \in G^{r}(h)} f(\tilde{g})=\int_{G} f(\tilde{g}) d \lambda^{r(h)}(\tilde{g}) .
$$

Given a Haar system $\left\{\lambda^{x}\right\}$, we define for $x \in G^{(0)}$ the measure $\lambda_{x}$ by $\lambda_{x}(E)=\lambda^{x}\left(E^{-1}\right)$, for every $E \subset G$ measureable.

Lemma 4.1.4. If $G$ is a locally compact Hausdorff étale groupoid, and $\lambda^{x}$ is the counting measure on $G^{x}$, then $\lambda_{x}$ is the counting measure on $G_{x}$.

Proof. Given a set $A,|A|$ denotes the number of elements in this set. Let $E \subset G$ measurable, 
then

$$
\begin{aligned}
\lambda_{x}(E) & =\lambda^{x}\left(E^{-1}\right) \\
& =\left|\left\{g: g \in E^{-1} \cap G^{x}\right\}\right| \\
& =\left|\left\{h^{-1}: h \in E \cap G_{x}\right\}\right|, \quad \text { by the change of variables } h^{-1}=g, \\
& =\left|\left\{h: h \in E \cap G_{x}\right\}\right|,
\end{aligned}
$$

since $h \mapsto h^{-1}$ is a bijection. Then $\lambda_{x}$ is the counting measure on $G_{x}$.

Definition 4.1.5. Let $G$ be a locally compact Hausdorff groupoid with a Haar system $\left\{\lambda^{x}\right\}_{x \in G^{(0)}}$. Given a Radon measure $\mu$ on $G^{(0)}$, we define the induced measures on $G$ by

$$
\nu(E)=\int_{G^{(0)}} \lambda^{x}(E) d \mu(x), \quad \nu^{-1}(E)=\int_{G^{(0)}} \lambda_{x}(E) d \mu(x)
$$

for every Borel set $E$. Or equivalently, for every $f \in C_{c}(G)$,

$$
\int f(g) d \nu(g)=\int_{G^{(0)}} \int_{G^{x}} f(g) d \lambda^{x}(g) d \mu(x), \quad \int f(g) d \nu^{-1}(g)=\int_{G^{(0)}} \int_{G_{x}} f(g) d \lambda_{x}(g) d \mu(x) .
$$

We denote $\nu=\int_{G^{(0)}} \lambda^{x} d \mu(x), \nu^{-1}=\int_{G^{(0)}} \lambda_{x} d \mu(x)$.

If $G$ a is locally compact Hausdorff étale groupoid and endowed with a Haar system $\left\{\lambda^{x}\right\}$ such that each $\lambda^{x}$ is a counting measure on $G^{x}$, then we denote the induced measures $\nu$ and $\nu^{-1}$ by $\mu_{r}$ and $\mu_{s}$. In this case,

$$
\int f(g) d \mu_{r}(g)=\int_{G^{(0)}} \sum_{g \in G^{x}} f(g) d \mu(x), \quad \int f(g) d \mu_{s}(g)=\int_{G^{(0)}} \sum_{g \in G_{x}} f(g) d \mu(x) .
$$

Definition 4.1.6. Suppose $\mu$ is a measure on $G^{(0)}$. We say $\mu$ is quasi-invariant if $\nu$ and $\nu^{-1}$ are equivalent measures. In this case we take $\Delta: G \rightarrow(0, \infty)$ to be the Radon-Nikodym derivative $d \nu / d \nu^{-1}$.

Remark 4.1.7. It follows from [10, Theorem 3.72] that it is possible to choose $\Delta$ to be a 
homomorphism from $G$ to $(0, \infty)$, where $(0, \infty)$ is a group with respect to the product.

Proposition 4.1.8. 17 Let $G$ be a locally compact Hausdorff étale groupoid with Haar system $\left\{\lambda^{x}\right\}$ given by counting measures $\lambda^{x}$ on $G^{x}$. If $\mu$ is a quasi-invariant measure on $G^{(0)}$, then for $\mu$-a.e. $x$ and all $g \in G_{x}^{x}$, we have $\Delta(g)=1$.

Proof. Let $G^{\prime}$ be the isotropy bundle defined by $G^{\prime}=\cup_{x \in G^{(0)}} G_{x}^{x}$. Note that $G^{\prime}$ is closed in $G$. In fact, if $\left\{g_{i}\right\}$ is a sequence in $G^{\prime}$ converging to some $g$ in $G$, it follows from the continuity of $r$ and $s$ that $r\left(g_{i}\right) \rightarrow r(g)$ and $s\left(g_{i}\right) \rightarrow s(g)$. Since $r\left(g_{i}\right)=s\left(g_{i}\right)$ for every $i$, we have $r(g)=s(g)$, that is, $g \in G^{\prime}$. Therefore $G^{\prime}$ is measurable.

Let $f$ be a positive measurable function whose support lies in $G^{\prime}$. Then, by assumption,

$$
\int_{G} f(g) d \mu_{r}(g)=\int_{G} f(g) \Delta(g) d \mu_{s}(g) .
$$

It follows from the definition of $\mu_{r}$ that

$$
\begin{aligned}
\int_{G} f(g) d \mu_{r}(g) & =\int_{G^{(0)}} \sum_{g \in G^{x}} f(g) d \mu(x) \\
& =\int_{G^{(0)}} \sum_{g \in G_{x}^{x}} f(g) d \mu(x),
\end{aligned}
$$

since $f$ is supported of $G^{\prime}$. Note that $f \Delta$ is also supported on $G^{\prime}$. Then, by definition of $\mu_{s}$, we have

$$
\begin{aligned}
\int_{G} f(g) \Delta(g) d \mu_{s}(g) & =\int_{G^{(0)}} \sum_{g \in G_{x}} f(g) \Delta(g) d \mu(x) \\
& =\int_{G^{(0)}} \sum_{g \in G_{x}^{x}} f(g) \Delta(g) d \mu(x) .
\end{aligned}
$$

\footnotetext{
${ }^{1}$ Thanks Frausino!
} 
Applying 4.4 and 4.5 in 4.3, we have

$$
\int_{G^{(0)}} \sum_{g \in G_{x}^{x}}(\Delta(g)-1) f(g) d \mu(x)=0
$$

Define $B^{+}=\left\{g \in G^{\prime}: \Delta(g) \geq 1\right\}$ and $B^{-}=\left\{g \in G^{\prime}: \Delta(g) \leq 1\right\}$. If we choose $f=\chi_{B^{+}}$, we have

$$
\int_{G^{(0)}} \sum_{g \in G_{x}^{x} \cap B^{+}}(\Delta(g)-1) d \mu(x)=0 .
$$

Since the function $x \mapsto \sum_{g \in G_{x}^{x} \cap B^{+}}(\Delta(g)-1)$ is non-negative, it follows that for $\mu$-a.e. $x$,

$$
\sum_{g \in G_{x}^{x} \cap B^{+}}(\Delta(g)-1)=0 .
$$

From the definition of $B^{+}$, for $\mu$-a.e. $x$ and all $g \in G_{x}^{x}$, if $\Delta(g) \geq 1$, it follows that $\Delta(g)=1$. Analogously for $B^{-}$, for $\mu$-a.e. $x$ and all $g \in G_{x}^{x}$, if $\Delta(g) \leq 1$, it follows that $\Delta(g)=1$. Therefore, for $\mu$-a.e. $x$ and all $g \in G_{x}^{x}$, we have $\Delta(g)=1$.

Remark 4.1.9. When we study Neshveyev's second theorem, we will consider measures $\mu$ such that $\Delta(g)=e^{c}$ for a continuous $\mathbb{R}$-valued 1-cocycle $c: G \rightarrow \mathbb{R}$. It follows from

Proposition 4.1 .8 that for $\mu$-a.e. $x \in G^{(0)}$, all $g \in G_{x}^{x}$, we have $c(g)=0$. In Remark 6.3 .22 on page 200, we prove this result for extremal $e^{\beta F}$-measures using the properties of the Renault-Deaconu groupoid.

\subsection{Borel Hilbert Bundles}

Given a group $G$, [18] defines a unitary representation of $G$ as a pair $(L, \mathcal{H})$, where $\mathcal{H}$ is a complex Hilbert space and $L$ is homomorphism from $G$ to the group of unitary operators on $\mathcal{H}$, usually written $\mathcal{U}(\mathcal{H})$, with product as group operation. We will denote $L_{g}$ as the image of $g$ in $G$ under the map $L$. 
We want to extend this notion to groupoids. By definition of groupoids, two elements are not necessarily composable. In order to define a unitary representation of groupoids, we will consider unitary operators $L_{g}: \mathcal{H}_{s(g)} \rightarrow \mathcal{H}_{r(g)}$, where $\left\{\mathcal{H}_{x}\right\}_{x \in G^{(0)}}$ is a family of Hilbert spaces indexed by $G^{(0)}$ and satisfying certain conditions.

Given this family of Hilbert spaces, we define a Hilbert space $L^{2}(X * \mathfrak{H}, \mu)$, also denoted by $\int_{X}^{\oplus} \mathcal{H}_{x} d \mu(x)$. By Renault's Disintegration Theorem, a representation $\pi: C_{c}(G) \rightarrow \mathcal{H}$ corresponds to a groupoid representation. Moreover, $\mathcal{H}$ can be identified with $\int_{X}^{\oplus} \mathcal{H}_{x} d \mu(x)$.

In this section we use results from [27], [10], [4], and [15].

Let $\mathfrak{H}=\left\{\mathcal{H}_{x}\right\}_{x \in X}$ be a collection of separable (nonzero) complex Hilbert spaces indexed by a locally compact Hausdorff second countable space $X$. Then the total space is defined by

$$
X * \mathfrak{H}=\left\{(x, h): h \in \mathcal{H}_{x}\right\}
$$

and we let $p: X * \mathfrak{H} \rightarrow X$ be defined by $p(x, h)=x$.

Remark 4.2.1. The total space $X * \mathfrak{H}$ is defined in [27] assuming $X$ is analytic. However, we will always assume that $X$ is locally compact Hausdorff second countable without loss of generality. Theorem 5.3 in [13] shows that every locally compact Hausdorff second countable space is analytic. More details about analytic and Polish spaces can be found in [6].

A section of $X * \mathfrak{H}$ is a function $f: X \rightarrow X * \mathfrak{H}$ such that $(p \circ f)(x)=x$ for each $x \in X$. The total space can be seen as the union of Hilbert spaces such that each $\mathcal{H}_{x}$ is glued to a point $x \in X$. A section maps $x$ to some point in $\{x\} \times \mathcal{H}_{x}$, as shown in Figure 4.1.

Example 4.2.2. Let $\mathcal{H}_{x}=\mathbb{C}$ for every $x \in X$. Then $X * \mathfrak{H}=X \times \mathbb{C}$. In this case, each section $\tilde{f}$ corresponds to a complex-valued function $f$ such that $\tilde{f}(x)=(x, f(x))$. Then we can identify $\tilde{f}$ with $f$ without loss of generality.

Given a Borel measure $\mu$ on $X$, we want to define a space of square-integrable sections, which we denote by $L^{2}(X * \mathfrak{H}, \mu)$, such that it is a Hilbert space and every section $f \in$ 


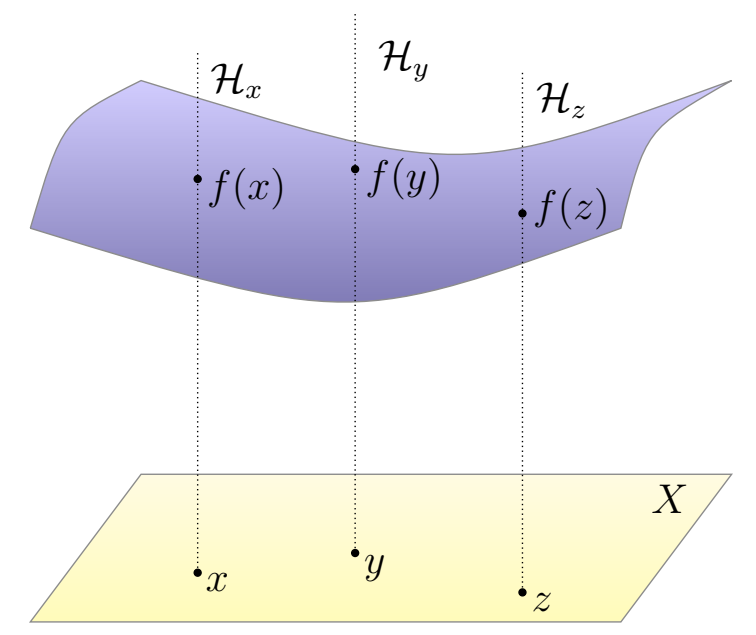

Figure 4.1: $X * \mathfrak{H}$ can be seen as a union of Hilbert spaces $\mathcal{H}_{x}$ such that every $\mathcal{H}_{x}$ corresponds to a point $x$ in $X$. A section is a function that maps $x$ to an element of $\{x\} \times \mathcal{H}_{x}$.

$L^{2}(X * \mathfrak{H}, \mu)$ satisfies

$$
\int_{X}\|f(x)\|^{2} d \mu(x)<\infty .
$$

Here we identify functions that are equal $\mu$-a.e.

If we endow $X * \mathfrak{H}$ with a Borel structure, we would naturally be interested in a subset of measurable sections, denoted by $\operatorname{Sec}(X * \mathfrak{H})$ such that

$$
x \mapsto\left\langle f_{1}(x), f_{2}(x)\right\rangle \text { is measurable for every } f_{1}, f_{2} \in \operatorname{Sec}(X * \mathfrak{H}),
$$

since we want to define the inner product on $L^{2}(X * \mathfrak{H})$ as

$$
\left\langle f_{1}, f_{2}\right\rangle=\int_{X}\left\langle f_{1}(x), f_{2}(x)\right\rangle d \mu(x) .
$$

We will replace condition (4.6) by simpler conditions which consider a countable family of sections. Later we will see that for every pair of measurable functions, (4.6) holds.

Definition 4.2.3. Let $\mathfrak{H}=\left\{\mathcal{H}_{x}\right\}_{x \in X}$ be a family of separable Hilbert spaces indexed by a locally compact Hausdorff second countable space $X$. Then $(X * \mathfrak{H}, p)$ is an analytic Borel 
Hilbert bundle if $X * \mathfrak{H}$ has a Borel structure such that

(a) For every $E \subset X, p^{-1}(E)$ is a Borel in $X * \mathfrak{H}$ if, and only if, $E$ is Borel in $X$;

(b) There is a sequence of sections $\left\{f_{n}\right\}$ such that

(i) the map $\tilde{f}_{n}: X * \mathfrak{H} \rightarrow \mathbb{C}$ defined by $\tilde{f}_{n}(x, h)=\left\langle f_{n}(x), h\right\rangle_{\mathcal{H}_{x}}$ is Borel for each $n$,

(ii) for each $n$ and $m, x \mapsto\left\langle f_{n}(x), f_{m}(x)\right\rangle_{\mathcal{H}_{x}}$ is Borel, and

(iii) the functions $\left\{\tilde{f}_{n}\right\}$ and $p$ separate points of $X * \mathfrak{H}$.

The sequence $\left\{f_{n}\right\}$ is called a fundamental sequence for $(X * \mathfrak{H}, p)$.

Remark 4.2.4. Given an analytic Borel Hilbert bundle $(X, p)$, we let $\operatorname{Sec}(X * \mathfrak{H})$ denote the set of sections $f: X \rightarrow X * \mathfrak{H}$ such that

$$
x \mapsto\left\langle f(x), f_{n}(x)\right\rangle
$$

is Borel for every $f_{n}$. It follows from [27, Remark F.3] that a section $f$ of $X * \mathfrak{H}$ is in $\operatorname{Sec}(X * \mathfrak{H})$ if, and only if, it is Borel.

Remark 4.2.5. Note that given a section $f$ of $X * \mathfrak{H}, f(x)$ is an element of $\{x\} \times \mathcal{H}_{x}$. Although this is an abuse of notation, we will identify $\{x\} \times \mathcal{H}_{x}$ with $\mathcal{H}_{x}$. Hence, the inner product $\langle f(x), h\rangle$ is well-defined for $h \in \mathcal{H}_{x}$. We can also write $\left\langle f_{1}(x), f_{2}(x)\right\rangle$ for sections $f_{1}, f_{2}$ without further comments.

Example 4.2.6. Let $X$ be a locally compact Hausdorff second countable space. Let $\mathcal{H}$ be a separable Hilbert space. Define $\mathcal{H}_{x}=\mathcal{H}$ for each $x \in X$. Then $X * \mathcal{H}=X \times \mathcal{H}$. Endow $X \times \mathcal{H}$ with the product topology. Given $E \subset X, p^{-1}(E)=E \times \mathcal{H}$. Thus $p^{-1}(E)$ is Borel if, and only if, $E$ is Borel. Therefore property (a) of Definition 4.2.3 holds.

Assume $\left\{e_{n}\right\}$ is an orthonormal basis for $\mathcal{H}$. Define a sequence of sections of $X * \mathfrak{H}$ by $f_{n}(x)=\left(x, e_{n}\right) \in\{x\} \times \mathcal{H}_{x}$. We will prove that $\left\{f_{n}\right\}$ is a fundamental sequence: 
(i) $\tilde{f}_{n}: X \times \mathcal{H} \rightarrow \mathbb{C}$ is defined by $\tilde{f}_{n}(x, h)=\left\langle f_{n}(x), h\right\rangle=\left\langle e_{n}, h\right\rangle$. $\tilde{f}_{n}$ is continuous, then it is Borel.

(ii) Given $n, m$, the function $x \mapsto\left\langle f_{n}(x), f_{m}(x)\right\rangle=\left\langle e_{n}, e_{m}\right\rangle$ is constant, therefore this function is Borel.

(iii) Let $(x, h),(y, k) \in X \times \mathcal{H}$. If $x \neq y$, then $p(x, h) \neq p(y, k)$ by definition of $p$. Assume $x=y$ and $h \neq k$. There exists $e_{n}$ such that $\left\langle e_{n}, h\right\rangle \neq\left\langle e_{n}, k\right\rangle$. Thus, $\tilde{f}_{n}(x, h) \neq \tilde{f}_{n}(y, k)$.

Therefore $(X \times \mathcal{H}, p)$ is an analytic Borel Hilbert bundle.

Example 4.2.7. Given a locally compact Hausdorff second countable space $X$, let $X=$ $X_{\infty} \cup X_{1} \cup X_{2} \cup \ldots$ be a Borel partition of $X$, i.e., every $X_{d}$ is Borel and the collection of $X_{d}$ is disjoint.

For every $d=\infty, 1,2, \ldots$, let $\mathcal{H}^{(d)}$ be a Hilbert space of dimension $d$ and basis $\left\{e_{n}^{d}\right\}_{n=1}^{n=d}$. Then

$$
X * \mathfrak{H}=\bigcup_{d=1}^{d=\infty} X_{d} \times \mathcal{H}^{(d)}
$$

Endow $X * \mathfrak{H}$ with a Borel structure such that $E \subset X * \mathfrak{H}$ is Borel if, and only if, $E \cap\left(X_{d} \times \mathcal{H}^{(d)}\right)$ is Borel for all $d$.

Define, for every $n=\infty, 1,2, \ldots$, the section $f_{n}$ such that

$$
f_{n}(x)= \begin{cases}e_{n}^{d}, & \text { if } x \in X_{d} \text { and } 1 \leq n \leq d \\ 0, & \text { otherwise }\end{cases}
$$

We prove that $(X * \mathfrak{H}, p)$ is an analytic Borel Hilbert bundle. Recall from Example 4.2.6 that $\left(X_{d} \times \mathcal{H}^{(d)},\left.p\right|_{X_{d}}\right)$ is an analytic Borel Hilbert bundle. Moreover, $\left\{\left.f_{n}\right|_{X_{d}}\right\}$ is a fundamental sequence. Here we assume $\left.f_{n}\right|_{X_{d}}: X_{d} \rightarrow X_{d} \times \mathcal{H}^{(d)}$ and $\left.p\right|_{X_{d} \times \mathcal{H}^{(d)}}: X_{d} \times \mathcal{H}^{(d)} \rightarrow X_{d}$ without loss of generality.

Now we show that all properties in Definition 4.2 .3 hold for $(X * \mathfrak{H}, p)$. 
(a) Let $E \subset X$. Then

$$
p^{-1}(E)=\bigcup_{d=1}^{d=\infty}\left(E \cap X_{d}\right) \times \mathcal{H}^{(d)}
$$

Assume $E$ is Borel and fix $d=\infty, 1,2, \ldots$ Then $E \cap X_{d}$ is a Borel subset of $X_{d}$. Since $\left(X_{d} \times \mathcal{H}^{(d)},\left.p\right|_{X_{d} \times \mathcal{H}^{(d)}}\right)$ is a Borel Hilbert bundle, it follows that

$$
\left.p\right|_{X_{d} \times \mathcal{H}^{(d)}} ^{-1}\left(E \cap X_{d}\right)=\left(E \cap X_{d}\right) \times \mathcal{H}^{(d)} \text { is Borel. }
$$

Note that $d$ is arbitrary. From 4.7 we have that $p^{-1}(E)$ is Borel.

Conversely, assume $p^{-1}(E)$ is Borel. Fix $d=\infty, 1,2, \ldots$ Then $p^{-1}(E) \cap\left(X_{d} \times \mathcal{H}^{(d)}\right)$ is Borel in $X_{d} \times \mathcal{H}^{(d)}$. However, from 4.7$), p^{-1}(E) \cap\left(X_{d} \times \mathcal{H}^{(d)}\right)=\left(E \cap X_{d}\right) \times \mathcal{H}^{(d)}$. Hence $E \cap X_{d}$ is Borel in $X$. Since $d$ is arbitrary, it follows that $E$ is Borel.

(b) (i) Fix $n .\left.\tilde{f}_{n}\right|_{X_{d}}$ is Borel for every $n, d$ from Example 4.2 .6 . Since $\widetilde{f}_{n}=\left.\widetilde{f}_{n}\right|_{X_{\infty}}+\left.\widetilde{f}_{n}\right|_{X_{1}}+\left.\widetilde{f}_{n}\right|_{X_{2}}+\ldots$, it follows that $\widetilde{f}_{n}$ is Borel.

(ii) Fix $d$ and let $n, m \in\{\infty, 1,2, \ldots\} .\left\{\left.f_{n}\right|_{X_{d}}\right\}$ is a fundamental sequence for the Borel Hilbert bundle $\left(X_{d} \times \mathcal{H}^{(d)},\left.p\right|_{X_{d} \times \mathcal{H}^{(d)}}\right)$. Then the function from $X_{d}$ to $\mathbb{C}$ defined by $x \mapsto\left\langle f_{n}(x), f_{m}(x)\right\rangle$ is Borel.

Since $d$ is arbitrary, the function from $X$ to $\mathbb{C}$ defined by $x \mapsto\left\langle f_{n}(x), f_{m}(x)\right\rangle$ is Borel.

(iii) Let $(x, h),(y, k) \in X * \mathfrak{H}$. If $x \neq y, p(x, h)=p(y, k)$.

Assume $x=y$. Let $d$ be such that $x \in X_{d}$. Since $\left(X_{d} \times \mathcal{H}^{(d)},\left.p\right|_{X_{d} \times \mathcal{H}^{(d)}}\right)$ is an analytic Borel Hilbert bundle with fundamental sequence $\left\{\left.f_{n}\right|_{X_{d} \times \mathcal{H}^{(d)}}\right\}_{n=1}^{n=\infty}$, there exists $n$ such that $\left.\tilde{f}_{n}\right|_{X_{d} \times \mathcal{H}^{(d)}}(x, h) \neq\left.\tilde{f}_{n}\right|_{X_{d} \times \mathcal{H}^{(d)}}(y, h)$. Thus $\tilde{f}_{n}(x, h) \neq \tilde{f}_{n}(x, k)$.

Therefore $(X * \mathfrak{H}, p)$ is an analytic Borel Hilbert bundle.

Given $f_{1}, f_{2} \in \operatorname{Sec}(X * \mathfrak{H})$, [27, Proposition F.6] shows that the function $x \mapsto\left\langle f_{1}(x), f_{2}(x)\right\rangle$ is Borel. If $\mu$ is a Borel measure on $X$, we can define the normed vector space $L^{2}(X * \mathfrak{H}, \mu)$ 
formed by the quotient of

$$
\mathcal{L}^{2}(X * \mathfrak{H}, \mu)=\left\{f \in \operatorname{Sec}(X * \mathfrak{H}): x \rightarrow\|f(x)\|^{2} \text { is } \mu \text {-integrable }\right\}
$$

where functions agreeing $\mu$-almost everywhere are identified. $L^{2}(X * \mathfrak{H}, \mu)$ is a Hilbert space with inner product defined by

$$
\left\langle f_{1}, f_{2}\right\rangle=\int_{X}\left\langle f_{1}(x), f_{2}(x)\right\rangle d \mu(x)
$$

Here we also denote $L^{2}(X * \mathfrak{H})$ by $\int_{X}^{\oplus} \mathcal{H}_{x} d \mu(x)$.

Given a Borel Hilbert bundle $X * \mathfrak{H}$, we can define a groupoid, called the isomorphism bundle, consisting of unitary operators between Hilbert spaces in the family $\left\{\mathcal{H}_{x}\right\}$.

Definition 4.2.8. Given $X$ a locally compact Hausdorff second countable space, let $(X * \mathfrak{H}, p)$ be an analytic Borel Hilbert bundle. The isomorphism bundle of $X * \mathfrak{H}$ is the set

$$
\operatorname{Iso}(X * \mathfrak{H})=\left\{(x, L, y) \text { such that } L: \mathcal{H}_{y} \rightarrow \mathcal{H}_{x} \text { is unitary }\right\}
$$

endowed with the smallest Borel structure such that, for all $f_{1}, f_{2} \in \operatorname{Sec}(X * \mathfrak{H})$,

$$
\phi_{f_{1}, f_{2}}(x, L, y)=\left\langle L f_{1}(y), f_{2}(x)\right\rangle
$$

define Borel functions from $\operatorname{Iso}(X * \mathfrak{H})$ to $\mathbb{C}$.

Lemma 4.2.9. Iso $(X * \mathfrak{H})$ is a groupoid whose units are defined by $\left(x, i d_{\mathcal{H}_{x}}, x\right)$. Two elements $(x, L, y)$ and $\left(y^{\prime}, M, z\right)$ are composable if $y=y^{\prime}$. In this case, we define their product by $(x, L, y)(y, M, z)=(x, L M, z)$. The inverse in $\operatorname{Iso}(X * \mathfrak{H})$ is defined by $(x, L, y)^{-1}=\left(y, L^{*}, x\right)$. The range and source maps are defined by $r(x, L, y)=\left(x, i d_{\mathcal{H}_{x}}, x\right)$ and $s(x, L, y)=\left(y, i d_{\mathcal{H}_{y}}, y\right)$ Proof. Given $x \in X, i d_{\mathcal{H}_{x}}$ is unitary. Hence $\left(x, i d_{\mathcal{H}_{x}}, x\right) \in \operatorname{Iso}(X * \mathfrak{H})$. Then $r, s$ are surjective. Note that we can identify $\left(x, i d_{\mathcal{H}_{x}}, x\right)$ with $x$ without loss of generality. Thus we will assume 
$X=\operatorname{Iso}(X * \mathfrak{H})^{(0)}$.

Now we show that the product in $\operatorname{Iso}(X * \mathfrak{H})$ is well-defined. In fact, given $(x, L, y)$, $(y, M, z) \in \operatorname{Iso}(X * \mathfrak{H})$, then $L M: \mathcal{H}_{z} \rightarrow \mathcal{H}_{x}$ is a unitary operator. Thus $(x, L M, z) \in$ $\operatorname{Iso}(X * \mathfrak{H})$. The inverse is well-defined as well, since $(x, L, y)^{-1}=\left(y, L^{*}, x\right)$ by definition and $L^{*}: \mathcal{H}_{x} \rightarrow \mathcal{H}_{y}$ is unitary.

Now we prove the conditions in Definition 3.1.1. Let $g=(x, L, y), h=(y, M, z)$ and $k=(z, N, w)$ be elements of $\operatorname{Iso}(X * \mathfrak{H})$. Then

(i) $s(g h)=s((x, L, y)(y, M, z))=s(x, L M, z)=z=s(y, M, z)=s(h)$, and

$$
r(g h)=r((x, L, y)(y, M, z))=r(x, L M, z)=x=r(x, L, y)=r(g) .
$$

(ii) $x=r\left(x, i d_{\mathcal{H}_{x}}, x\right)=r(x)$, and $x=s\left(x, i d_{\mathcal{H}_{x}}, x\right)=s(x)$.

(iii) $g s(g)=g y=(x, L, y)\left(y, i d_{\mathcal{H}_{y}}, y\right)=\left(x, \operatorname{Lid}_{\mathcal{H}_{y}}, y\right)=(x, L, y)=g$, and

$$
r(g) g=x g=\left(x, i d_{\mathcal{H}_{x}}, x\right)(x, L, y)=\left(x, i d_{\mathcal{H}_{x}} L, y\right)=(x, L, y)=g
$$

(iv) $(g h) k=[(x, L, y)(y, M, z)](z, N, w)=(x, L M, z)(z, N, w)=(x, L M N, w)$, and $g(h k)=(x, L, y)[(y, M, z)(z, N, w)]=(x, L, y)(y, M N, w)=(x, L M N, w)$.

Then $(g h) k=g(h k)$.

(v) $g g^{-1}=(x, L, y)\left(y, L^{*}, x\right)=\left(x, L L^{*}, x\right)=\left(x, i d_{\mathcal{H}_{x}}, x\right)=x=r(g)$, and

$$
g^{-1} g=\left(y, L^{*}, x\right)(x, L, y)=\left(y, L^{*} L, y\right)=\left(y, i d_{\mathcal{H}_{y}}, y\right)=y=s(g) .
$$

Therefore $\operatorname{Iso}(X * \mathfrak{H})$ is a groupoid.

Definition 4.2.10. Let $G$ be a locally compact Hausdorff second countable étale groupoid with Haar system $\left\{\lambda^{x}\right\}_{x \in G^{(0)}}$. A unitary representation of $G$ is a triple $\left(\mu, G^{(0)} * \mathfrak{H}, L\right)$ such that $\mu$ is a quasi-invariant measure on $G^{(0)}, L=\left\{L_{g}\right\}_{g \in G}$ is a family of unitary operators $L_{g}: \mathcal{H}_{s(g)} \rightarrow \mathcal{H}_{r(g)}$, and $\widehat{L}: G \rightarrow \operatorname{Iso}\left(G^{(0)} * \mathfrak{H}\right)$ is a Borel homomorphism such that $g \mapsto \widehat{L}_{g}=$ $\left(r(g), L_{g}, s(g)\right)$. 
Now we define a norm on $C_{c}(G)$ which will be an upper bound for the norm of a representation obtained with the Renault's disintegration theorem. This norm is defined in [20].

Definition 4.2.11. Let $G$ be a locally compact Hausdorff second countable étale groupoid with Haar system $\left\{\lambda^{x}\right\}_{x \in G^{(0)}}$. We define the $I$-norm on $C_{c}(G)$ by

$$
\|f\|_{I}=\max \left\{\sup _{x \in G^{(0)}} \int_{G}|f(g)| d \lambda^{x}(g), \sup _{x \in G^{(0)}} \int_{G}|f(g)| d \lambda_{x}(g)\right\} .
$$

If the Haar system is given by counting measures $\lambda^{x}$ on $G^{x}$, then

$$
\|f\|_{I}=\max \left\{\sup _{x \in G^{(0)}} \sum_{g \in G^{x}}|f(g)|, \sup _{x \in G^{(0)}} \sum_{g \in G_{x}}|f(g)|\right\} .
$$

Lemma 4.2.12. Let $G$ be a locally compact Hausdorff second countable étale groupoid with Haar system given by counting measures $\lambda^{x}$ on $G^{x}$. Then $\|f\|_{I}<\infty$ for every $f \in C_{c}(G)$. Moreover, $\|\cdot\|_{I}$ defines a norm on $C_{c}(G)$.

Proof. Let $f_{1}, f_{2}, f \in C_{c}(G), \lambda \in \mathbb{C}$.

- $\|f\|_{I}=0$ implies $f=0$.

Assume $\|f\|_{I}=0$. Let $g \in G$ and $x=r(g)$. Then,

$$
|f(g)| \leq \sum_{h \in G^{x}}|f(h)| \leq\|f\|_{I}=0 .
$$

Therefore $f=0$.

- $\|\lambda f\|=|\lambda|\|f\|$.

Let $x \in G^{(0)}$. Then

$$
\sum_{g \in G^{x}}|\lambda f(g)|=|\lambda| \sum_{g \in G^{x}}|f(g)|
$$


Thus,

$$
\sup _{x \in G^{(0)}} \sum_{g \in G^{x}}|\lambda f(g)|=|\lambda| \sup _{x \in G^{(0)}} \sum_{g \in G^{x}}|f(g)| .
$$

Analogously,

$$
\sup _{x \in G^{(0)}} \sum_{g \in G_{x}}|\lambda f(g)|=|\lambda| \sup _{x \in G^{(0)}} \sum_{g \in G_{x}}|f(g)| .
$$

Therefore $\|\lambda f\|_{I}=|\lambda|\|f\|_{I}$..

- $\left\|f_{1}+f_{2}\right\|_{I} \leq\left\|f_{1}\right\|_{I}+\left\|f_{2}\right\|_{I}$.

$$
\begin{aligned}
\left\|f_{1}+f_{2}\right\|_{I} & =\max \left\{\sup _{x \in G^{(0)}} \sum_{g \in G^{x}}\left|f_{1}(g)+f_{2}(g)\right|, \sup _{x \in G^{(0)}} \sum_{g \in G_{x}}\left|f_{1}(g)+f_{2}(g)\right|\right\} \\
& \leq \max \left\{\sup _{x \in G^{(0)}} \sum_{g \in G^{x}}\left(\left|f_{1}(g)\right|+\left|f_{2}(g)\right|\right), \sup _{x \in G^{(0)}} \sum_{g \in G_{x}}\left(\left|f_{1}(g)\right|+\left|f_{2}(g)\right|\right)\right\} \\
& \leq \max \left\{\sup _{x \in G^{(0)}} \sum_{g \in G^{x}}\left|f_{1}(g)\right|+\left\|f_{2}\right\|_{I}, \sup _{x \in G^{(0)}} \sum_{g \in G_{x}}\left|f_{1}(g)\right|+\left\|f_{2}\right\|_{I}\right\} \\
& =\left\|f_{1}\right\|_{I}+\left\|f_{2}\right\|_{I} .
\end{aligned}
$$

Now we show that $\|f\|_{I}<\infty$ for every $f \in C_{c}(G)$.

Let $\mathcal{U}$ be an open bisection of $G$ and let $f \in C_{c}(\mathcal{U})$. For every $x \in r(\mathcal{U})$, there is a unique $h^{x} \in \mathcal{U}$ satisfying $r\left(h^{x}\right)=x$. Note that $x=\left.r\right|_{\mathcal{U}} ^{-1}(x)$. Hence,

$$
\sum_{g \in G^{x}}|f(g)|=\left|f\left(h^{x}\right)\right| .
$$


Since $\left.r\right|_{\mathcal{U}}$ is a homeomorphism, we have

$$
\sup _{x \in r(\mathcal{U})} \sum_{g \in G^{x}}|f(g)|=\sup _{x \in r(\mathcal{U})}\left|f\left(h^{x}\right)\right|=\sup _{x \in r(\mathcal{U})}|f \circ r|_{\mathcal{U}}^{-1}(x)\left|=\sup _{g \in \mathcal{U}}\right| f(g) \mid=\|f\|_{\infty} .
$$

If $x \notin r(\mathcal{U})$, then $f(g)=0$ for every $g \in G^{x}$. Thus,

$$
\sup _{x \in G^{(0)}} \sum_{g \in G^{x}}|f(g)|=\|f\|_{\infty} .
$$

Analogously, we can prove that

$$
\sup _{x \in G^{(0)}} \sum_{g \in G_{x}}|f(g)|=\|f\|_{\infty} .
$$

Therefore $\|f\|_{I}=\|f\|_{\infty}$ for $f \in C_{c}(\mathcal{U})$.

Now let $f \in C_{c}(G)$. It follows from Proposition 3.3 .5 that there exist $f_{1}, \ldots f_{n}$ continuous functions supported on open bisections such that $f=f_{1}+\ldots+f_{n}$. Then $\|f\|_{I} \leq\left\|f_{1}\right\|+$ $\ldots+\left\|f_{n}\right\|<\infty$. Therefore $\|\cdot\|_{I}$ is a norm on $C_{c}(G)$.

\subsection{Renault's Disintegration Theorem}

The results in this section can be found in [15]. The Renault's Disintegration Theorem can also be found in [4] and [10. However, both use the abstract notion of upper semi-continuous $\mathrm{C}^{*}$-bundle and the theorem is stated in a more general case than the results presented here.

Proposition 4.3.1. Let $G$ be a locally compact Hausdorff étale groupoid with Haar system $\left\{\lambda^{x}\right\}_{x \in G^{(0)}}$. If $\left(\mu, G^{(0)} * \mathfrak{H}, L\right)$ is a unitary representation of $G$, then we obtain a $\|\cdot\|_{I^{-}}$ norm bounded representation $\pi$ of $C_{c}(G)$ on $\int_{G^{(0)}}^{\oplus} \mathcal{H}_{x} d \mu(x)$, called the integrated form of 
$\left(\mu, G^{(0)} * \mathfrak{H}, L\right)$, determined by

$$
\langle\pi(f) h, k\rangle=\int_{G} f(g)\left\langle L_{g} h_{s(g)}, k_{r(g)}\right\rangle \Delta(g)^{-\frac{1}{2}} d \nu(g),
$$

where $\nu=\int_{G^{(0)}} d \lambda^{x} d \mu(x), \nu^{-1}=\int_{G^{(0)}} d \lambda_{x} d \mu(x)$ and $\Delta=d \nu / d \nu^{-1}$ as described in Definition 4.1 .5 .

Renault's disintegration theorem shows that a representation $\pi: C_{c}(G) \rightarrow \mathcal{H}$ can be identified with a groupoid representation $\left(\mu, G^{(0)} * \mathfrak{H}, L\right)$ such that $\widehat{L}(G)$ is measurable in $\operatorname{Iso}\left(G^{(0)} * \mathfrak{H}\right)$. Condition 4.8 on page 88 will be necessary in order to write 4.9 ).

Definition 4.3.2. Given a vector space $\mathcal{H}_{0}, \operatorname{Lin}\left(\mathcal{H}_{0}\right)$ denotes the set of linear operators $T: \mathcal{H}_{0} \rightarrow \mathcal{H}_{0}$. Note that $\operatorname{Lin}\left(\mathcal{H}_{0}\right)$ is an algebra whose product is defined by composition of operators.

Theorem 4.3.3. (Renault's disintegration theorem) Let $G$ be a locally compact Hausdorff étale groupoid. Suppose that $\mathcal{H}_{0}$ is a dense subspace of a complex Hilbert space $\mathcal{H}$. Let $\pi: C_{c}(G) \rightarrow \operatorname{Lin}\left(\mathcal{H}_{0}\right)$ be a homomorphism such that:

(a) $\left\{\pi(f) h: f \in C_{c}(G)\right.$ and $\left.h \in \mathcal{H}_{0}\right\}$ is dense in $\mathcal{H}$;

(b) For each $h, k \in \mathcal{H}_{0}$,

$$
f \mapsto\langle\pi(f) h, k\rangle
$$

is continuous in the inductive limit topology on $C_{c}(G)$;

(c) For $f \in C_{c}(G)$ and $h, k \in \mathcal{H}_{0}$, we have

$$
\langle\pi(f) h, k\rangle=\left\langle h, \pi\left(f^{*}\right) k\right\rangle .
$$

Then each $\pi(f)$ is bounded and extends to an operator $\bar{\pi}(f)$ on $\mathcal{H}$ of norm at most $\|f\|_{I}$. Furthermore, $\bar{\pi}$ is a representation of $C_{c}(G)$ on $\mathcal{H}$ and there is a unitary representation 
$\left(\mu, G^{(0)} * \mathfrak{H}, L\right)$ of $G$ such that $\mathcal{H} \sim L^{2}\left(G^{(0)} * \mathfrak{H}, \mu\right)$ and $\bar{\pi}$ is equivalent to the integrated form of $\left(\mu, G^{(0)} * \mathfrak{H}, L\right)$.

Remark 4.3.4. Recall that the inductive limit topology is introduced in Definition 3.3 .18 on page 71 .

Lemma 4.3.5. Let $G$ be a locally compact Hausdorff second countable étale groupoid. Let $\varphi$ be a state on $C^{*}(G)$ with GNS-triple $(\mathcal{H}, \pi, \xi)$. Then $\pi$ satisfies the conditions of Renault's disintegration theorem.

Proof. Assume $\varphi$ is a state on $C^{*}(G)$. Let $(\mathcal{H}, \pi, \xi)$ be the corresponding GNS-triple.

(a) $\left\{\pi(f) h: f \in C_{c}(G)\right.$ and $\left.h \in \mathcal{H}\right\}$ is dense in $\mathcal{H}$ Since $\xi$ is a cyclic vector, it follows that $\pi\left(C^{*}(G)\right) \xi$ is dense in $\mathcal{H}$. Recall that $C_{c}(G)$ is dense in $C^{*}(G)$, then $\pi\left(C_{c}(G)\right) \xi$ is dense in $\pi\left(C^{*}(G)\right) \xi$. Therefore, the property holds.

(b) For $h, k \in \mathcal{H}, f \mapsto\langle\pi(f) h, k\rangle$ is continuous in the inductive limit topology on $C_{c}(G)$ Let $h, k \in \mathcal{H}$. Given $f \in C_{c}(G)$, let $\left\{f_{i}\right\}_{i \in \mathbb{N}}$ be a sequence in $C_{c}(G)$ such that $f_{i} \rightarrow f$ with respect to the inductive limit topology. It follows from Lemma 3.3 .19 that $f_{i} \rightarrow f$ in $C^{*}(G)$. Then $\left\|\pi\left(f_{i}\right)-\pi(f)\right\| \rightarrow 0$ and therefore $\left\langle\left(\pi\left(f_{i}\right)-\pi(f)\right) k, h\right\rangle \rightarrow 0$.

(c) $\langle\pi(f) h, k\rangle=\left\langle h, \pi\left(f^{*}\right)\right\rangle$

Let $h, k \in \mathcal{H}, f \in C_{c}(G)$. Since $\pi$ is an *-representation of $C^{*}(G)$, we have $\pi\left(f^{*}\right)=$ $\pi(f)^{*}$. Therefore the property holds. 


\section{Chapter 5}

\section{Neshveyev's Theorems}

In this chapter we describe the KMS states of a groupoid $\mathrm{C}^{*}$-algebra. Neshveyev proves this result in [17] in two theorems: the first theorem describes all states satisfying a certain condition, and the second theorem describes all KMS states and it is a corollary of the first result. These theorems show a correspondence between a KMS state $\varphi$ on $C^{*}(G)$ and a pair $\left(\mu,\left\{\varphi_{x}\right\}_{x \in G^{(0)}}\right)$ consisting of a probability measure $\mu$ on $G^{(0)}$ and a family of states $\varphi_{x}$ on $C^{*}\left(G_{x}^{x}\right)$ satisfying a certain condition.

Before proving Neshveyev's theorems, we define KMS states on a $\mathrm{C}^{*}$-algebra as described in [2], [3] and [9].

\subsection{KMS States}

In this section we define the notion of KMS states and prove some of their main properties. The results in this section can be found in [2], [3] and [9]. KMS states characterizes the equilibrium states in quantum statistical mechanics.

Before we define KMS states, let us recall the definition of approximate unit and prove some properties of states on a $\mathrm{C}^{*}$-algebra.

Let $A$ be a $C^{*}$-algebra. An approximate unit for $A$ is an increasing net $\left\{u_{\lambda}\right\}_{\lambda \in \Lambda}$ of positive 
elements in the closed unit ball of $A$ such that $a=\lim _{\lambda} a u_{\lambda}$ for all $a \in A$. Equivalently, $a=\lim _{\lambda} u_{\lambda} a$. From [16], it follows that every $\mathrm{C}^{*}$-algebra $A$ contains an approximate unit. Moreover, if $A$ is separable, then it admits an approximate unit which is a sequence.

Recall the following theorems on positive linear functionals from [16]:

Theorem 5.1.1. If $\varphi$ is a positive linear functional on a $\mathrm{C}^{*}$-algebra $A$, then it is bounded.

Theorem 5.1.2. Let $\varphi$ be a bounded linear functional on a $\mathrm{C}^{*}$-algebra $A$. The following conditions are equivalent:

(i) $\varphi$ is positive.

(ii) For each approximate unit $\left\{u_{\lambda}\right\}_{\lambda \in \Lambda},\|\varphi\|=\lim _{\lambda} \varphi\left(u_{\lambda}\right)$.

(iii) For some approximate unit $\left\{u_{\lambda}\right\}_{\lambda \in \Lambda},\|\varphi\|=\lim _{\lambda} \varphi\left(u_{\lambda}\right)$.

Proof. Assume $\varphi \neq 0$. First we show the implication $(i) \Rightarrow(i i)$ holds.

Note that the map $A^{2} \rightarrow \mathbb{C}$ defined by $(a, b) \mapsto \varphi\left(b^{*} a\right)$ is a positive sesquilinear form on $A$. Hence $\varphi\left(b^{*} a\right)=\overline{\varphi\left(a^{*} b\right)}$ and the Cauchy-Schwarz inequality $\left|\varphi\left(b^{*} a\right)\right| \leq \varphi\left(b^{*} b\right)^{1 / 2} \varphi\left(a^{*} a\right)^{1 / 2}$ holds.

Let $\left(u_{\lambda}\right)_{\lambda \in \Lambda}$ be an approximate unit of $A$ and let $a \in A$ with $a \neq 0$. Then, for every $\lambda \in \Lambda$,

$$
\left|\varphi\left(a u_{\lambda}\right)\right|^{2} \leq \varphi\left(a^{*} a\right) \varphi\left(u_{\lambda}^{*} u_{\lambda}\right)=\varphi\left(a^{*} a\right) \varphi\left(u_{\lambda}^{2}\right)
$$

by the Cauchy-Schwarz inequality. Since the net $\left\{u_{\lambda}\right\}_{\lambda \in \Lambda}$ is increasing and its elements are in the unit ball, then the net $\left\{u_{\lambda}^{2}\right\}_{\lambda \in \Lambda}$ is also increasing and included in the unit ball. Because $\varphi$ is positive and bounded, we have $\sup _{\lambda \in \Lambda} \varphi\left(u_{\lambda}^{2}\right)=\lim _{\lambda \in \Lambda} \varphi\left(u_{\lambda}^{2}\right)$. Then, for every $i \in \Lambda$, we have by 5.1 ,

$$
\left|\varphi\left(a u_{i}\right)\right|^{2} \leq \varphi\left(a^{*} a\right) \lim _{\lambda \in \Lambda} \varphi\left(u_{\lambda}^{2}\right) \leq\|\varphi\|\left\|a^{*} a\right\| \lim _{\lambda \in \Lambda} \varphi\left(u_{\lambda}^{2}\right)=\|\varphi\|\|a\|^{2} \lim _{\lambda \in \Lambda} \varphi\left(u_{\lambda}^{2}\right) .
$$


Therefore, using the continuity of $\varphi$,

$$
\begin{array}{r}
\lim _{\lambda \in \Lambda}\left|\varphi\left(a u_{i}\right)\right|^{2} \leq\|\varphi\|\|a\|^{2} \lim _{\lambda \in \Lambda} \varphi\left(u_{\lambda}^{2}\right) \\
|\varphi(a)|^{2} \leq\|\varphi\|\|a\|^{2} \lim _{\lambda \in \Lambda} \varphi\left(u_{\lambda}^{2}\right) \\
\frac{|\varphi(a)|^{2}}{\|a\|^{2}} \leq\|\varphi\| \lim _{\lambda \in \Lambda} \varphi\left(u_{\lambda}^{2}\right) .
\end{array}
$$

Since $a$ is arbitrary, $\|\varphi\| \leq \lim _{\lambda \in \Lambda} \varphi\left(u_{\lambda}^{2}\right)$. On the other hand, for each $\lambda \in \Lambda$,

$$
\varphi\left(u_{\lambda}^{2}\right) \leq\|\varphi\|\left\|u_{\lambda}^{2}\right\| \leq\|\varphi\|
$$

Then $\|\varphi\|=\lim _{\lambda \in \Lambda} \varphi\left(u_{\lambda}^{2}\right)$. Note that $u_{\lambda}^{2}-u_{\lambda} \leq 0$ for every $\lambda$, thus $\varphi\left(u_{\lambda}^{2}\right) \leq \varphi\left(u_{\lambda}\right)$. Therefore,

$$
\|\varphi\|=\lim _{\lambda \in \Lambda} \varphi\left(u_{\lambda}^{2}\right) \leq \lim _{\lambda \in \Lambda} \varphi\left(u_{\lambda}\right) \leq\|\varphi\|
$$

It is obvious that $(i i) \Rightarrow(i i i)$.

Now we show that $(i i i) \Rightarrow(i)$. Suppose that $\left\{u_{n}\right\}_{n \in \mathbb{N}}$ is an approximate unit such that $1=\lim _{n \rightarrow \infty} \tau\left(u_{n}\right)$. Let $a$ be a selft-adjoint element of $A$ such that $\|a\| \leq 1$ and write $\tau(a)=\alpha+i \beta$ where $\alpha, \beta$ are real numbers. To show that $\tau(a) \in \mathbb{R}$, we may suppose that $\beta \leq 0$. If $k$ is a positive integer, then

$$
\begin{aligned}
\left\|a-i u_{\lambda}\right\|^{2} & =\left\|\left(a+i k u_{n}\right)\left(a-i k u_{n}\right)\right\| \\
& =\left\|a^{2}+k^{2} u_{n}^{2}-i k\left(a u_{n}-u_{n} a\right)\right\| \\
& \leq 1+k^{2}+k\left\|a u_{n}-u_{n} a\right\|,
\end{aligned}
$$

so $\left|\tau\left(a-i k u_{n}\right)\right|^{2} \leq 1+k^{2}+k\left\|a u_{n}-u_{n} a\right\|$ because $\|\tau\|=1$.

However, $\lim _{k \rightarrow \infty} \tau\left(a-i k u_{n}\right)=\tau(a)-i k$ by hypothesis, and $\lim _{k \rightarrow \infty}\left(a u_{n}-u_{n} a\right)=0$, so 
in the limit as $n \rightarrow \infty$, we get

$$
|\alpha+i \beta-i k|^{2}=|\tau(a)-i k|^{2}=|\alpha+i \beta-i k|^{2} \leq 1+k^{2},
$$

then

$$
\begin{aligned}
& \alpha^{2}+\beta^{2}-2 k \beta+k^{2} \leq 1+n^{2} \\
\Rightarrow & -2 k \beta \leq 1-\beta^{2}-\alpha^{2} .
\end{aligned}
$$

Since $\beta$ is not positive and this inequality holds for all positive integers $n, \beta$ must be zero. Therefore, $\tau(a)$ is real if $a$ is hermitian.

Now suppose that $a$ is positive and $\|a\| \leq 1$. Then $u_{n}-a$ is hermitian and $\left\|u_{n}-a\right\| \leq 1$, so $\tau\left(u_{n}-a\right) \leq 1$. But then $1-\tau(a)=\lim _{n \rightarrow \infty} \tau\left(u_{n}-a\right) \leq 1$, and therefore $\tau(a) \geq 0$. Thus, $\tau$ is positive and we have shown (iii) $\Rightarrow(i)$.

We want to show that, if the $\mathrm{C}^{*}$-algebra $A$ is commutative and unital, then the extremal states are precisely the characters on $A$. We need some results on convex spaces before studying this.

Definition 5.1.3. Given a commutative $\mathrm{C}^{*}$-algebra $A$, a character is a non-zero homomorphism $\varphi: A \rightarrow \mathbb{C}$.

Definition 5.1.4. Let $X$ be a normed vector space. A functional $\eta$ in a convex subset $C \subset X^{*}$ is an extreme point in $C$ (or extremal) if the condition $\eta=t \eta_{1}+(1-t) \eta_{2}$, where $\eta_{1}, \eta_{2} \in C, 0<t<1$, implies that $\eta=\eta_{1}=\eta_{2}$.

Definition 5.1.5. We say that a state $\varphi$ on $A$ is pure if it has the property that whenever $\rho$ is a positive linear functional on $A$ such that $\rho \leq \varphi$, necessarily there is a number $t \in[0,1]$ such that $\rho=t \varphi$.

Theorem 5.1.6. [3, Theorem 2.3.15] Let $A$ be a unital $C^{*}$-algebra. The set of states on $A$ is convex and its extremal points are the pure states. 
Theorem 5.1.7. [16, Theorem 5.1.6] Let $\varphi$ be a state on a commutative $\mathrm{C}^{*}$-algebra $A$. Then $\varphi$ is pure if, and only if, it is a character on $A$.

Lemma 5.1.8. Let $X$ be a Borel space endowed with a probability measure $\mu$. Let $A$ be a separable $\mathrm{C}^{*}$-algebra and $A_{0}$ a dense subset of $A$. Let $\psi_{x}$ be a family of states on $A$ defined for $\mu$-a.e. $x \in X$ such that for every $a \in A_{0}$ the map $x \mapsto \psi_{x}(a)$ is $\mu$-measurable. Define $\varphi$ by

$$
\varphi(a)=\int_{X} \psi_{x}(a) d \mu(x)
$$

Then $\varphi$ is a state on $A$.

Proof. Let $V \subset X$ be a conull set such that $\psi_{x}$ is defined for every $x \in V$. Define for every $a \in A$ the function $F_{a}: X \rightarrow \mathbb{C}$ by

$$
F_{a}(x)= \begin{cases}\psi_{x}(a) & \text { if } x \in V \\ 0 & \text { otherwise }\end{cases}
$$

By assumption, $F_{a}$ is $\mu$-measurable for every $a \in A_{0}$.

Let $a \in A$. Since $A_{0}$ is dense in $A$, there exists a sequence $\left\{a_{n}\right\}_{n \in \mathbb{N}}$ in $A_{0}$ converging to $a$. Then, using the continuity of $\psi_{x}$, we have for $\mu$-a.e. $x$,

$$
F_{a}(x)=\psi_{x}(a)=\lim _{n \rightarrow \infty} \psi_{x}\left(a_{n}\right)=\lim _{n \rightarrow \infty} F_{a_{n}}(x)
$$

Therefore $F_{a}$ is measurable. In other words, the function $x \mapsto \psi_{x}(a)$ is $\mu$-measurable for every $a \in A$.

In order to show that $\varphi$ is a state, we begin by proving that $\varphi$ is a positive linear functional. Since each $\psi_{x}$ linear, it follows from the definition of $\varphi$ that $\varphi$ is also linear. Let $a$ be a positive 
element in $A$. Then $\psi_{x}(a) \geq 0$ for $\mu$-a.e. $x$. Thus,

$$
\varphi(a)=\int_{X} \psi_{x}(a) d \mu(x) \geq 0
$$

By Theorem 5.1.1, $\varphi$ is bounded. It follows from Theorem 5.1 .2 that, for some approximate unit $\left\{u_{n}\right\}_{n \in \mathbb{N}}$ of $A$,

$$
\|\varphi\|=\lim _{n \rightarrow \infty} \varphi\left(u_{n}\right)=\lim _{n \rightarrow \infty} \int_{X} \psi_{x}\left(u_{n}\right) d \mu(x) .
$$

However,

$$
\left|\psi_{x}\left(u_{n}\right)\right| \leq\left\|\psi_{x}\right\|\left\|u_{n}\right\|=\left\|u_{n}\right\| \leq 1
$$

for every $n$ and for $\mu$-a.e. $x \in X$ because each $\psi_{x}$ is a state and every $u_{n}$ satisfies $\left\|u_{n}\right\| \leq 1$. Therefore we can apply the dominated convergence theorem, then

$$
\begin{aligned}
\|\varphi\| & =\lim _{n \rightarrow \infty} \int_{X} \psi_{x}\left(u_{n}\right) d \mu(x) \\
& =\int_{X}\left(\lim _{n \rightarrow \infty} \psi_{x}\left(u_{n}\right)\right) d \mu(x) \\
& =\int_{X} 1 d \mu(x), \quad \text { since each } \psi_{x} \text { is a state, } \\
& =1 .
\end{aligned}
$$

Now we define the notion of dynamical system on a $\mathrm{C}^{*}$-algebra and prove some of its properties.

Definition 5.1.9. We say that $\tau=\left\{\tau_{t}\right\}_{t \in \mathbb{R}}$ is a one-parameter group of $*$-automorphisms of a $\mathrm{C}^{*}$-algebra $A$ if $\tau_{t}: A \rightarrow A$ is an $*$-automorphism and

(i) $\tau_{t+s}=\tau_{t} \circ \tau_{s}$ for all $t, s \in \mathbb{R}$; 
(ii) $\tau_{0}=i d$.

Example 5.1.10. Consider the algebra of square matrices $M_{n}(\mathbb{C})$ for some $n$. Let $H \in$ $M_{n}(\mathbb{C})$, and define each $\tau_{t}: M_{n}(\mathbb{C}) \rightarrow M_{n}(\mathbb{C})$ by $\tau_{t}(A)=e^{i t H} A e^{-i t H}$. Note that $\tau_{t}$ is linear and $\tau_{0}$ is the identity. Each $\tau_{t}$ is a homomorphism because

$$
\tau_{t}(A B)=e^{i t H} A B e^{-i t H}=e^{i t H} A e^{-i t H} e^{i t H} B e^{-i t H}=\tau_{t}(A) \tau_{t}(B)
$$

Also, the equality $\tau_{t+s}=\tau_{t} \circ \tau_{s}$ holds, since

$$
\tau_{t+s}(A)=e^{i(t+s) H} A e^{-i(t+s) H}=e^{i t H} e^{i s H} A e^{-i s H} e^{-i t H}=e^{i t H} \tau_{s}(A) e^{-i t H}=\tau_{t}\left(\tau_{s}(A)\right) .
$$

Definition 5.1.11. A $C^{*}$-dynamical system is a pair $(A, \tau)$ where $A$ is a $C^{*}$-algebra and $\tau=\left\{\tau_{t}\right\}_{t \in \mathbb{R}}$ is a one-parameter group of $*$-automorphisms strongly continuous, i.e., $t \mapsto \tau_{t}(a)$ is continuous in the norm for all $a \in A$. If we fix $\tau$, we say that $\tau$ is the dynamics on $A$.

Now we prove that, under certain conditions, if we define a one-parameter group of *automorphisms $\tau$ on a dense $*$-algebra of a $\mathrm{C}^{*}$-algebra $A$, then we can extend the operators uniquely on $A$. Moreover, $\tau$ defines a dynamics on $A$.

Lemma 5.1.12. Let $A_{0}$ be a dense *-subalgebra of a $C^{*}$-algebra $A$. Let $\tau=\left\{\tau_{t}\right\}_{t \in \mathbb{R}}$ be a family of $*$-automorphisms $\tau_{t}: A_{0} \rightarrow A_{0}$ such that

(i) $\tau_{t+s}=\tau_{t} \circ \tau_{s}$ for $t, s \in \mathbb{R}$,

(ii) $\tau_{0}=i d$,

(iii) $t \mapsto \tau_{t}(a)$ is continuous in the norm for each $a \in A_{0}$.

Moreover, assume

$$
\left\|\tau_{t}(a)\right\| \leq\|a\|, \quad \text { for every } t \in \mathbb{R}, a \in A_{0} .
$$


Then $\tau$ can be extended uniquely to a dynamics on $A$, which we also denote by $\tau=\left\{\tau_{t}\right\}_{t \in \mathbb{R}}$ without loss of generality.

Proof. First we show that each $\tau_{t}$ can be extended uniquely to $A$. Let $a \in A$. Since $A_{0}$ is dense in $A$, there exists a sequence $\left\{a_{n}\right\}_{n \in \mathbb{N}}$ converging to $a$.

Using the fact that $A_{0}$ is a vector space and the equation 5.2 , we have that $\left\{\tau_{t}\left(a_{n}\right)\right\}_{n \in \mathbb{N}}$ is a Cauchy sequence, then we can define $\tau_{t}(a)=\lim _{n \rightarrow \infty} \tau_{t}\left(a_{n}\right)$.

Note that $\tau_{t}(a)$ is well-defined. In fact, let $\left\{b_{n}\right\}_{n \in \mathbb{N}}$ be an arbitrary sequence converging to $a$, and let $x=\lim _{n \rightarrow \infty} \tau_{t}\left(b_{n}\right)$. Then

$$
\left\|\tau_{t}(a)-x\right\|=\lim _{n \rightarrow \infty}\left\|\tau_{t}\left(a_{n}\right)-\tau_{t}\left(b_{n}\right)\right\| \leq \lim _{n \rightarrow \infty}\left\|a_{n}-b_{n}\right\|=0
$$

It follows from the defintion of $\tau_{t}$ on $A$ that $\left\|\tau_{t}(a)\right\| \leq\|a\|$ for every $a \in A$.

Note that the extension $\tau$ also satisfies $\tau_{0}=i d$ and $\tau_{t+s}=\tau_{t} \circ \tau_{s}$. In fact, given $a \in A$, let $\left\{a_{n}\right\}_{n \in \mathbb{N}}$ be a sequence in $A_{0}$ converging to $a$. Then

$$
\begin{aligned}
\tau_{0}(a) & =\lim _{n \rightarrow \infty} \tau_{0}\left(a_{n}\right)=\lim _{n \rightarrow \infty} a_{n}=a \\
\tau_{t+s}(a) & =\lim _{n \rightarrow \infty} \tau_{t+s}\left(a_{n}\right)=\lim _{n \rightarrow \infty} \tau_{t} \circ \tau_{s}\left(a_{n}\right)=\tau_{t} \circ \tau_{s}(a) .
\end{aligned}
$$

Then $\tau_{t}$ is invertible with inverse $\tau_{-t}$.

Now we show that $\tau_{t}: A \rightarrow A$ is an automorphism. Let $a, b \in A$ and $\left\{a_{n}\right\}_{n \in \mathbb{N}},\left\{b_{n}\right\}_{n \in \mathbb{N}}$ sequences in $A_{0}$ converging to $a, b$ respectively. Then

$$
\begin{aligned}
& \tau_{t}(a b)=\lim _{n \rightarrow \infty} \tau_{t}\left(a_{n} b_{n}\right)=\lim _{n \rightarrow \infty} \tau_{t}\left(a_{n}\right) \tau_{t}\left(b_{n}\right)=\tau_{t}(a) \tau_{t}(b), \\
& \tau_{t}\left(a^{*}\right)=\lim _{n \rightarrow \infty} \tau_{t}\left(a_{n}^{*}\right)=\lim _{n \rightarrow \infty} \tau_{t}\left(a_{n}\right)^{*}=\tau_{t}(a)^{*}
\end{aligned}
$$

Finally, we show that $\tau$ is strongly continuous. Let $a \in A$ and fix $t_{0}$. Given $\varepsilon$, there exists $a_{0} \in A_{0}$ such that $\left\|a-a_{0}\right\|<\varepsilon / 3$. Let $\delta>0$ such that $\left\|\tau_{t}\left(a_{0}\right)-\tau_{s}\left(a_{0}\right)\right\|<\varepsilon / 3$ for every $t \in \mathbb{R}$ such that $\left|t-t_{0}\right|<\delta$. 
Given $t$ with $\left|t-t_{0}\right|<\delta$, we have

$$
\begin{aligned}
\left\|\tau_{t}(a)-\tau_{t_{0}}(a)\right\| & \leq\left\|\tau_{t}(a)-\tau_{t}\left(a_{0}\right)\right\|+\left\|\tau_{t}\left(a_{0}\right)-\tau_{t_{0}}\left(a_{0}\right)\right\|+\left\|\tau_{t_{0}}\left(a_{0}\right)-\tau_{t_{0}}(a)\right\| \\
& \leq 2\left\|a-a_{0}\right\|+\left\|\tau_{t}\left(a_{0}\right)-\tau_{t_{0}}\left(a_{0}\right)\right\| \\
& \leq 2 \frac{\varepsilon}{3}+\frac{\varepsilon}{3} \\
& =\varepsilon
\end{aligned}
$$

Let $X$ be a complex Banach space and $X^{*}$ its dual. Let $\sigma\left(X, X^{*}\right)$ denote the topology on $X$ induced by the functionals on $X$. This topology is denoted the weak topology on $X$.

Remark 5.1.13. We say that a function $f: \mathbb{R} \rightarrow X$ is $\sigma\left(X, X^{*}\right)$-continuous if $\eta \circ f: \mathbb{R} \rightarrow \mathbb{C}$ is continuous for every $\eta \in X^{*}$.

Definition 5.1.14. Given a Banach space $X$, let $\left\{\tau_{t}\right\}_{t \in \mathbb{R}}$ be a family of linear and bounded operators $\tau_{t}: X \rightarrow X$. This family is called a one-parameter $\sigma\left(X, X^{*}\right)$-continuous group of isometries if

(i) $\tau_{t+s}=\tau_{t} \circ \tau_{s}$ for all $t, s \in \mathbb{R}$;

(ii) $\tau_{0}=i d$;

(iii) $\left\|\tau_{t}\right\|=1$ for all $t \in \mathbb{R}$;

(iv) $t \mapsto \tau_{t}(a)$ is $\sigma\left(X, X^{*}\right)$-continuous for all $a \in X$.

Lemma 5.1.15. Let $A$ be a $\mathrm{C}^{*}$-algebra and $\tau$ its dynamics. Then $\tau$ is a one-parameter $\sigma\left(A, A^{*}\right)$-continuous group of isometries.

Proof. Note that properties (i) and (ii) in Definitions 5.1 .9 and 5.1 .14 are equal. In both, every $\tau$ is a linear operator on $A$. So we need to show properties (iii) and (iv) of Definition 5.1 .14 
(iii) $\left\|\tau_{t}\right\|=1$

This follows from the fact that every *-automorphism on a $\mathrm{C}^{*}$-algebra is an isometry.

(iv) $t \mapsto \tau_{t}(a)$ is $\sigma\left(A, A^{*}\right)$-continuous for all $a \in A$

Let $a \in A$ and $t_{0} \in \mathbb{R} . \tau$ is strongly continuous by assumption. Then $\tau_{t}(a) \rightarrow \tau_{t_{0}}(a)$ in the norm as $t \rightarrow t_{0}$. Thus, for every $\eta \in A^{*}, \eta\left(\tau_{t}(a)\right) \rightarrow \eta\left(\tau_{t_{0}}(a)\right)$ as $t \rightarrow t_{0}$. Thus property (iv) holds.

Therefore $\tau$ is a one-parameter $\sigma\left(A, A^{*}\right)$-continuous group of isometries.

Note that, for every $a \in X$, the function $t \mapsto \eta \circ \tau_{t}(a)$ is continuous for all $\eta \in X^{*}$. If we can extend this function to an analytic function on a strip in $\mathbb{C}$ which we will define later, we say that $a$ is analytic. We will prove that the set of analytic elements is dense in $X$.

Definition 5.1.16. Let $\tau$ be a one-parameter $\sigma\left(X, X^{*}\right)$-continuous group of isometries. An element $a \in X$ is analytic for $\tau$ if there exists $\lambda>0$ and a function $f: I_{\lambda} \rightarrow X$, where $I_{\lambda}=\{z \in \mathbb{C}:|\operatorname{Im}(z)|<\lambda\}$, such that

(i) $f(t)=\tau_{t}(a)$ for all $t \in \mathbb{R}$;

(ii) The function $z \mapsto \eta(f(z))$ is analytic in $I_{\lambda}$ for all $\eta \in X^{*}$.

Under these conditions we write

$$
\tau_{z}(a)=f(z), \text { for } z \in I_{\lambda}
$$

If $\lambda=\infty$, we say that $a$ is entire analytic for $\tau$.

Remark 5.1.17. Suppose $a_{1}, a_{2} \in X$ are entire analytic, and $\alpha \in \mathbb{C}$. Let $f_{1}, f_{2}$ be the corresponding functions as in Definition 5.1.16. Then, for every $t \in \mathbb{R}$,

$$
f_{1}(t)+\alpha f_{2}(t)=\tau_{t}\left(a_{1}\right)+\alpha \tau_{t}\left(a_{2}\right)=\tau_{t}\left(a_{1}+\alpha a_{2}\right) .
$$


Moreover, the function $z \mapsto \eta\left(f_{1}(z)+\alpha f_{2}(z)\right)$ is analytic for every $\eta \in X^{*}$. Thus $a_{1}+\alpha a_{2}$ is entire analytic. Therefore the set of entire analytic elements in $X$, denoted $X_{\tau}$, is a subspace of $X$. For $a_{1}, a_{2} \in X_{\tau}, \alpha, z \in \mathbb{C}$, it follows from (5.3) that $\tau_{z}\left(a_{1}+\alpha a_{2}\right)=\tau_{z}\left(a_{1}\right)+\tau_{z}\left(a_{2}\right)$.

Later we will prove that $X_{\tau}$ is also dense in $X$, both in the $\sigma\left(X, X^{*}\right)$-topology (see Proposition 5.1.19) and with respect to the norm (see Corollary 5.1.24).

When $\tau$ defines a dynamics on a $\mathrm{C}^{*}$-algebra $A, A_{\tau}$ is not only a vector space, but a *-subalgebra. We will prove this result in Lemma 5.1 .22 on page 110 .

Lemma 5.1.18. Let $n$ be a positive integer, and $\delta>0$. Then

$$
\begin{aligned}
\sqrt{\frac{n}{\pi}} \int_{-\infty}^{\infty} e^{-n t^{2}} d t & =1 \\
\sqrt{\frac{n}{\pi}} \int_{|t| \geq \delta} e^{-n t^{2}} d t & =\frac{1}{\sqrt{\pi}} \int_{|t| \geq \sqrt{n} \delta} e^{-t^{2}} d t .
\end{aligned}
$$

Moreover, the second integral converges to zero as $n \rightarrow \infty$.

Proof. Let $\delta \geq 0$. Since the function $t \mapsto e^{-n t^{2}}$ is even, we have

$$
\begin{aligned}
\sqrt{\frac{n}{\pi}} \int_{|t| \geq \delta} e^{-n t^{2}} d t & =2 \sqrt{\frac{n}{\pi}} \int_{t \geq \delta} e^{-n t^{2}} d t \\
& =\frac{2}{\sqrt{\pi}} \int_{s \geq \sqrt{n} \delta} e^{-s^{2}} d s, \quad \text { by the change of variables } s=\sqrt{n} t \\
& =\frac{1}{\sqrt{\pi}} \int_{|s| \geq \sqrt{n} \delta} e^{-s^{2}} d s
\end{aligned}
$$

Thus the equality (5.5) follows. If $\delta=0$, we have

$$
\sqrt{\frac{n}{\pi}} \int_{-\infty}^{\infty} e^{-n t^{2}} d t=\sqrt{\frac{n}{\pi}} \int_{|t| \geq 0} e^{-n t^{2}} d t=\frac{1}{\sqrt{\pi}} \int_{|s| \geq 0} e^{-s^{2}} d s=\frac{1}{\sqrt{\pi}} \int_{-\infty}^{\infty} e^{-s^{2}} d s=1
$$

Therefore the integral $\sqrt{\frac{n}{\pi}} \int_{|t| \geq \delta} e^{-n t^{2}} d t$ converges to zero as $n \rightarrow \infty$.

Proposition 5.1.19. If $\tau$ is a one-parameter $\sigma\left(X, X^{*}\right)$-continuous group of isometries, and 
$a \in X$, define for $n=1,2, \ldots$,

$$
a_{n}=\sqrt{\frac{n}{\pi}} \int_{-\infty}^{\infty} \tau_{t}(a) e^{-n t^{2}} d t
$$

Then, for each $n, a_{n}$ is an entire analytic element for $\tau$ and $\left\|a_{n}\right\| \leq\|a\|$. In addition,

$$
\tau_{z}\left(a_{n}\right)=\sqrt{\frac{n}{\pi}} \int_{-\infty}^{\infty} \tau_{t}(a) e^{-n(t-z)^{2}} d t
$$

for every $z \in \mathbb{C}$. Moreover, $a_{n} \rightarrow a$ on the $\sigma\left(X, X^{*}\right)$ topology as $n \rightarrow \infty$. In particular, $X_{\tau}$, is a $\sigma\left(X, X^{*}\right)$-dense subspace of $X$.

Proof. Let $a \in X$. Define for each $n=1,2, \ldots$ the function $f_{n}: \mathbb{C} \rightarrow X$ by

$$
f_{n}(z)=\sqrt{\frac{n}{\pi}} \int_{-\infty}^{\infty} \tau_{t}(a) e^{-n(t-z)^{2}} d t
$$

This function is well-defined since $t \mapsto e^{-n(t-z)^{2}}$ is an integrable function, $\left\|\tau_{t}(a)\right\| \leq\|a\|$ for every $t$, and $t \mapsto e^{-n(t-z)^{2}} \tau_{t}(a)$ is continuous, then we apply Proposition 2.6. Note that for each $s \in \mathbb{R}$, we have

$$
\begin{aligned}
f_{n}(s) & =\sqrt{\frac{n}{\pi}} \int_{-\infty}^{\infty} \tau_{t}(a) e^{-n(t-s)^{2}} d t \\
& =\sqrt{\frac{n}{\pi}} \int_{-\infty}^{\infty} \tau_{t+s}(a) e^{-n t^{2}} d t \\
& =\sqrt{\frac{n}{\pi}} \int_{-\infty}^{\infty} \tau_{s} \circ \tau_{t}(a) e^{-n t^{2}} d t \\
& =\tau_{s}\left(\sqrt{\frac{n}{\pi}} \int_{-\infty}^{\infty} \tau_{t}(a) e^{-n t^{2}} d t\right), \quad \text { by Corollary 2.6.17, } \\
& =\tau_{s}\left(a_{n}\right) .
\end{aligned}
$$

Let $\eta \in X^{*}$. Let $z, z_{0} \in \mathbb{C}$ such that $z \neq z_{0}$. By definition of $f_{n}$, it follows that

$$
\left|\frac{\eta\left(f_{n}(z)\right)-\eta\left(f_{n}\left(z_{0}\right)\right)}{z-z_{0}}-\sqrt{\frac{n}{\pi}} \int_{-\infty}^{\infty} 2 n(t-z) e^{-n(t-z)^{2}} \eta\left(\tau_{t}(a)\right) d t\right|
$$


equals to

$$
\begin{aligned}
& \left|\sqrt{\frac{n}{\pi}} \int_{-\infty}^{\infty} \eta\left(\tau_{t}(a)\right)\left(\frac{e^{-n(t-z)^{2}}-e^{-n\left(t-z_{0}\right)^{2}}}{z-z_{0}}\right) d t-\sqrt{\frac{n}{\pi}} \int_{-\infty}^{\infty} 2 n(t-z) e^{-n(t-z)^{2}} \eta\left(\tau_{t}(a)\right) d t\right| \\
= & \sqrt{\frac{n}{\pi}}\left|\int_{-\infty}^{\infty}\left(\frac{e^{-n(t-z)^{2}}-e^{-n\left(t-z_{0}\right)^{2}}}{z-z_{0}}-2 n(t-z) e^{-n(t-z)^{2}}\right) \eta\left(\tau_{t}(a)\right) d t\right| \\
\leq & \sqrt{\frac{n}{\pi}} \int_{-\infty}^{\infty}\left|\frac{e^{-n(t-z)^{2}}-e^{-n\left(t-z_{0}\right)^{2}}}{z-z_{0}}-2 n(t-z) e^{-n(t-z)^{2}}\right|\left|\eta\left(\tau_{t}(a)\right)\right| d t \\
\leq & \|\eta\|\|a\| \sqrt{\frac{n}{\pi}} \int_{-\infty}^{\infty}\left|\frac{e^{-n(t-z)^{2}}-e^{-n\left(t-z_{0}\right)^{2}}}{z-z_{0}}-2 n(t-z) e^{-n(t-z)^{2}}\right| d t,
\end{aligned}
$$

since $\left|\eta\left(\tau_{t}(a)\right)\right| \leq\|\eta\|\left\|\tau_{t}(a)\right\| \leq\|\eta\|\|a\|$ for each $t$. This integral goes to zero when $z \rightarrow z_{0}$ and the entire analyticity follows. Also, $f_{n}(z)=\tau_{z}\left(a_{n}\right)$ for every $z \in \mathbb{C}$.

In addition, we have $\left\|a_{n}\right\| \leq\|a\|$. In fact,

$$
\begin{aligned}
\left\|a_{n}\right\| & =\left\|\sqrt{\frac{n}{\pi}} \int_{-\infty}^{\infty} \tau_{t}(a) e^{-n t^{2}} d t\right\| \\
& \leq \sqrt{\frac{n}{\pi}} \int_{-\infty}^{\infty}\left\|\tau_{t}(a)\right\| e^{-n t^{2}} d t, \text { from Lemma 2.6.11, } \\
& \leq\|a\| \sqrt{\frac{n}{\pi}} \int_{-\infty}^{\infty} e^{-n t^{2}} d t \\
& =\|a\|, \quad \text { from Lemma 5.1.18. }
\end{aligned}
$$

Now we prove that $a_{n}$ converges to $a$ on the $\sigma\left(X, X^{*}\right)$ topology. Let $\eta \in X^{*}$. If $\eta=0$ or $a=0$, it follows that $\eta\left(a_{n}\right)=\eta(a)=0$ for every $n$. Then we assume $\eta \neq 0$ and $a \neq 0$ without loss of generality.

Note that

$$
\begin{aligned}
\eta\left(a_{n}-a\right) & =\eta\left(a_{n}\right)-\eta(a) \\
& =\sqrt{\frac{n}{\pi}} \int_{-\infty}^{\infty} \eta\left(\tau_{t}(a)\right) e^{-n t^{2}} d t-\eta(a), \quad \text { by definition of } a_{n}
\end{aligned}
$$




$$
=\sqrt{\frac{n}{\pi}} \int_{-\infty}^{\infty}\left(\eta\left(\tau_{t}(a)\right)-\eta(a)\right) e^{-n t^{2}} d t
$$

since $\sqrt{\frac{n}{\pi}} \int_{-\infty}^{\infty} e^{-n t^{2}} d t=1$ by Lemma 5.1.18.

Let $\varepsilon>0$. Since the map $t \mapsto \tau_{t}(a)$ is continuous and $\eta \in X^{*}$, there exists $\delta>0$ such that

$$
|t|<\delta \text { implies }\left|\eta\left(\tau_{t}(a)\right)-\eta(a)\right|<\varepsilon / 2 \text {. }
$$

Moreover, from Lemma 5.1.18, there exists $N>0$ such that, for every $n \geq N$, we have

$$
\sqrt{\frac{n}{\pi}} \int_{|t| \geq \delta} e^{-n t^{2}} d t<\frac{\varepsilon}{4\|\eta\|\|a\|}
$$

Then, for each $n \geq N$,

$$
\begin{aligned}
\left|\eta\left(a_{n}-a\right)\right| & =\left|\sqrt{\frac{n}{\pi}} \int_{-\infty}^{\infty} e^{-n t^{2}}\left(\eta\left(\tau_{t}(a)\right)-\eta(a)\right) d t\right| \\
& \leq\left|\sqrt{\frac{n}{\pi}} \int_{|t| \geq \delta} e^{-n t^{2}}\left(\eta\left(\tau_{t}(a)\right)-\eta(a)\right) d t\right|+\left|\sqrt{\frac{n}{\pi}} \int_{|t|<\delta} e^{-n t^{2}}\left(\eta\left(\tau_{t}(a)\right)-\eta(a)\right) d t\right| \\
& \left.\left.\leq \sqrt{\frac{n}{\pi}} \int_{|t| \geq \delta} e^{-n t^{2}} \mid \eta\left(\tau_{t}(a)\right)-\eta(a)\right)\left|d t+\sqrt{\frac{n}{\pi}} \int_{|t|<\delta} e^{-n t^{2}}\right| \eta\left(\tau_{t}(a)\right)-\eta(a)\right) \mid d t \\
& \left.\leq \sqrt{\frac{n}{\pi}} \int_{|t| \geq \delta} e^{-n t^{2}} \mid \eta\left(\tau_{t}(a)\right)-\eta(a)\right) \mid d t+\frac{\varepsilon}{2} \sqrt{\frac{n}{\pi}} \int_{|t|<\delta} e^{-n t^{2}} d t, \quad \text { from (5.6), } \\
& \leq \sqrt{\frac{n}{\pi}} \int_{|t| \geq \delta} e^{-n t^{2}}\|\eta\|\left(\left\|\tau_{t}(a)\right\|+\|a\|\right) d t+\frac{\varepsilon}{2} \sqrt{\frac{n}{\pi}} \int_{|t|<\delta} e^{-n t^{2}} d t \\
& \leq \sqrt{\frac{n}{\pi}} \int_{|t| \geq \delta} e^{-n t^{2}}\|\eta\|(\|a\|+\|a\|) d t+\frac{\varepsilon}{2} \sqrt{\frac{n}{\pi}} \int_{|t|<\delta} e^{-n t^{2}} d t, \quad \text { since }\left\|\tau_{t}\right\|=1, \\
& =2\|\eta\|\|a\| \sqrt{\frac{n}{\pi}} \int_{|t| \geq \delta} e^{-n t^{2}} d t+\frac{\varepsilon}{2} \sqrt{\frac{n}{\pi}} \int_{|t|<\delta} e^{-n t^{2}} d t \\
& \leq 2\|\eta\|\|a\| \frac{\varepsilon}{4\|\eta\|\|a\|}+\frac{\varepsilon}{2} \sqrt{\frac{n}{\pi}} \int_{|t|<\delta} e^{-n t^{2}} d t, \quad \text { from }(\underline{5.7)}, \\
& \leq 2\|a\| \frac{\varepsilon}{4\|\eta\|\|a\|}+\frac{\varepsilon}{2} \sqrt{\frac{n}{\pi}} \int_{-\infty}^{\infty} e^{-n t^{2}} d t,
\end{aligned}
$$




$$
\begin{aligned}
& =\frac{\varepsilon}{2}+\frac{\varepsilon}{2}, \text { from Lemma 5.1.18, } \\
& =\varepsilon .
\end{aligned}
$$

Hence $a_{n} \rightarrow a$ in the topology $\sigma\left(X, X^{*}\right)$.

Remark 5.1.20. Let $\tau$ be a one-parameter $\sigma\left(X, X^{*}\right)$-continuous group of isometries. Given $\lambda$, we say that $a$ is analytic on the strip $I_{\lambda}$ if the conditions in Definition 5.1.16 hold for $a$ and $\lambda$.

We say that $a \in X$ is strongly analytic on the strip $I_{\lambda}$ if there exists $f: I_{\lambda} \rightarrow X$ such that condition (i) in Definition 5.1.16 is satisfied and the limit

$$
\lim _{h \rightarrow 0} \frac{f(z+h)-f(z)}{h}
$$

exists for every $z \in I_{\lambda}$. We now show that these two notions are equivalent.

Lemma 5.1.21. Let $\tau$ be a one-parameter $\sigma\left(X, X^{*}\right)$-continuous group of isometries, and $a \in X_{\tau}$. Given $w \in \mathbb{C}, \tau_{w}(a) \in X_{\tau}$. Moreover, for every $z \in \mathbb{C}$ we have

$$
\tau_{z+w}(a)=\tau_{z} \circ \tau_{w}(a)
$$

Proof. Let $a \in X_{\tau}$. Then there exists a function $f: \mathbb{C} \rightarrow X$ such that $f(t)=\tau_{t}(a)$ for every $t \in \mathbb{R}$, and $\eta \circ f$ is entire analytic for $\eta \in X^{*}$.

Fix $s \in \mathbb{R}$. Let $f_{s}: \mathbb{C} \rightarrow X$ be the function defined by $f_{s}(w)=f(s+w)$. Then $\eta \circ f_{s}$ is entire analytic for all $\eta \in X^{*}$.

Define $g: \mathbb{C} \rightarrow X$ by $g(w)=\tau_{s} \circ \tau_{w}(a)$. Note that $\eta \circ g$ is entire analytic for every $\eta \in X^{*}$. In fact, given $w \in \mathbb{C}$,

$$
\eta \circ g(w)=\eta \circ \tau_{s} \circ \tau_{w}(a)=\left(\eta \circ \tau_{s}\right) \circ f(z)
$$

Since $\eta \circ \tau_{s} \in X^{*}$ and $a$ is entire analytic, it follows that $\left(\eta \circ \tau_{s}\right) \circ f$ is entire analytic. Thus 
$\eta \circ g$ is entire analytic. Moreover, for $t \in \mathbb{R}$, we have

$$
\eta \circ g(t)=\eta \circ \tau_{s} \circ \tau_{t}(a)=\eta \circ \tau_{s+t}(a)=\eta \circ f(t+s)=\eta \circ f_{s}(t)
$$

Thus $\eta \circ g$ and $\eta \circ f_{s}$ are entire analytic functions which agree on the real line. Thus, by the uniqueness theorem for analytic functions [1, Theorem 6.9] we have $\eta \circ g=\eta \circ f_{s}$. $\eta$ is arbitrary and $X^{*}$ separates poins in $X$, thus $g=f_{s}$. Therefore, for every $w \in \mathbb{C}$,

$$
\tau_{s} \circ \tau_{w}(a)=\tau_{s+w}(a)
$$

Now we fix $w \in \mathbb{C}$ and assume $s$ is a real variable. Define $f_{w}: \mathbb{C} \rightarrow X$ by $f_{w}(z)=f(w+z)$. Since $a$ is entire analytic, the function $\eta \circ f_{w}$ is entire analytic for every $\eta \in X^{*}$. By equation (5.8), we have for all $t \in \mathbb{R}$ the equality

$$
f_{w}(t)=f(w+t)=\tau_{t+w}(a)=\tau_{t} \circ \tau_{w}(a) .
$$

Therefore $\tau_{w}(a)$ is entire analytic and

$$
\tau_{z} \circ \tau_{w}(a)=f_{w}(z)=f(w+z)=\tau_{z+w}(a) .
$$

Let $\tau$ define a dynamics on a $\mathrm{C}^{*}$-algebra. We say that a subset $A_{1}$ is $\tau$-invariant if $\tau_{t}(a) \in A_{1}$ for every $t \in \mathbb{R}, a \in A_{1}$.

Lemma 5.1.22. Let $(A, \tau)$ be a $\mathrm{C}^{*}$-dynamical system. Then $A_{\tau}$ is a $*$-subalgebra which is $\tau$-invariant.

Proof. $A_{\tau}$ is a vector space by Remark 5.1.17. Moreover, $A_{\tau}$ is $\tau$-invariant by Lemma 5.1.21.

Given $a, b \in A_{\tau}$, let $f_{1}, f_{2}: \mathbb{C} \rightarrow A$ be the functions such that $\eta \circ f_{1}, \eta \circ f_{2}$ are analytic for all $\eta \in A^{*}$, and such that $f_{1}(t)=\tau_{t}(a), f_{2}(t)=\tau_{t}(b)$ for every $t \in \mathbb{R}$. 
Let $f=f_{1} f_{2}$. Then $\eta \circ f$ is analytic for all $\eta \in A^{*}$. Moreover, given $t \in \mathbb{R}$,

$$
f(t)=f_{1}(t) f_{2}(t)=\tau_{t}(a) \tau_{t}(b)=\tau_{t}(a b) .
$$

Therefore $a b$ is entire analytic.

Define $f_{1}^{*}$ by $f_{1} *(z)=f_{1}(\bar{z})^{*}$. Then, for $t \in \mathbb{R}$,

$$
f_{1}^{*}(t)=f_{1}(t)^{*}=\tau_{t}(a)^{*}=\tau_{t}\left(a^{*}\right)
$$

Given $\eta \in A^{*}$, let $\eta_{1} \in A^{*}$ be defined by $\eta_{1}(b)=\overline{\eta\left(b^{*}\right)}$ for $b \in A$. Then,

$$
\eta \circ f_{1}^{*}(z)=\eta\left(f_{1}(\bar{z})^{*}\right)=\overline{\eta_{1} \circ f(\bar{z})}
$$

Note that the function $z \mapsto \overline{\eta_{1} \circ f_{1}(\bar{z})}$ is analytic. In fact,

$$
\lim _{h \rightarrow 0} \frac{\overline{\eta_{1} \circ f_{1}(\bar{z}+\bar{h})}-\overline{\eta_{1} \circ f_{1}(\bar{z})}}{h}=\overline{\left(\lim _{h \rightarrow 0} \frac{\eta_{1} \circ f_{1}(\bar{z}+\bar{h})-\eta_{1} \circ f_{1}(\bar{z})}{\bar{h}}\right)} .
$$

This limit exists since $\eta_{1} \circ f_{1}$ is analytic. Then $\eta \circ f_{1}^{*}$ is analytic, and therefore $a^{*}$ is entire analytic.

Proposition 5.1.23. Let $\tau$ be a one-parameter $\sigma\left(X, X^{*}\right)$-continuous group of isometries. Given $\lambda>0, a \in X$ is analytic on the strip $I_{\lambda}$ if, and only if, $a$ is strongly analytic on $I_{\lambda}$.

Proof. Let $\eta \in X^{*}$. Let $z_{0} \in I_{\lambda}$. There exists $r>0$ such that $D\left(z_{0}, r / 2\right) \subset \overline{D\left(z_{0}, r\right)} \subset I_{\lambda}$.

Let $h, k \in D(0, r / 2)$. Then $z_{0}+h, z_{0}+k \in D\left(z_{0}, r / 2\right)$. Using Cauchy's integral formula and assuming $C=\partial D\left(z_{0}, r\right)$, we have

$$
\begin{aligned}
\eta\left(f\left(z_{0}\right)\right) & =\frac{1}{2 \pi i} \oint_{C} \frac{\eta(f(z))}{z-z_{0}} d z \\
\eta\left(f\left(z_{0}+h\right)\right) & =\frac{1}{2 \pi i} \oint_{C} \frac{\eta(f(z))}{z-z_{0}-h} d z \\
\eta\left(f\left(z_{0}+k\right)\right) & =\frac{1}{2 \pi i} \oint_{C} \frac{\eta(f(z))}{z-z_{0}-k} d z
\end{aligned}
$$


Then

$$
\begin{aligned}
& \eta\left(\frac{f\left(z_{0}+h\right)-f\left(z_{0}\right)}{h}-\frac{f\left(z_{0}+k\right)-f\left(z_{0}\right)}{k}\right) \\
= & \frac{1}{2 \pi i} \oint_{C} \eta(f(z))\left[\frac{1}{h}\left(\frac{1}{z-z_{0}-h}-\frac{1}{z-z_{0}}\right)-\frac{1}{k}\left(\frac{1}{z-z_{0}-k}-\frac{1}{z-z_{0}}\right)\right] d z
\end{aligned}
$$

Note that $\frac{1}{a-b}-\frac{1}{a}=\frac{b}{a(a-b)}$ for $a, b$ complex numbers such that $a \neq b$ and $a \neq 0$. Applying this, we have that (5.9) equals to

$$
\begin{aligned}
& \frac{1}{2 \pi i} \oint_{C} \eta(f(z))\left[\frac{1}{h} \frac{h}{\left(z-z_{0}\right)\left(z-z_{0}-h\right)}-\frac{1}{k} \frac{k}{\left(z-z_{0}\right)\left(z-z_{0}-k\right)}\right] d z \\
= & \frac{1}{2 \pi i} \oint_{C} \eta(f(z))\left[\frac{1}{\left(z-z_{0}\right)\left(z-z_{0}-h\right)}-\frac{1}{\left(z-z_{0}\right)\left(z-z_{0}-k\right)}\right] d z \\
= & \frac{1}{2 \pi i} \oint_{C} \frac{\eta(f(z))}{z-z_{0}}\left[\frac{1}{z-z_{0}-h}-\frac{1}{z-z_{0}-k}\right] d z \\
= & \frac{1}{2 \pi i} \oint_{C} \frac{\eta(f(z))}{z-z_{0}} \frac{h-k}{\left(z-z_{0}-h\right)\left(z-z_{0}-k\right)} d z
\end{aligned}
$$

Note that for every $z$ such that $\left|z-z_{0}\right|=r$, we have

$$
\left|z-z_{0}-h\right| \geq\left|z-z_{0}\right|-|h| \geq r-\frac{r}{2}=\frac{r}{2}
$$

Thus

$$
\frac{1}{\left|z-z_{0}-h\right|} \leq \frac{2}{h}
$$

Analogously, $\frac{1}{\left|z-z_{0}-k\right|} \leq \frac{2}{k}$. Recall that $C$ denotes the set of points $z$ satisfying $\left|z-z_{0}\right|=r$. Therefore,

$$
\begin{aligned}
\left|\eta\left(\frac{f\left(z_{0}+h\right)-f\left(z_{0}\right)}{h}-\frac{f\left(z_{0}+k\right)-f\left(z_{0}\right)}{k}\right)\right| & =\left|\frac{1}{2 \pi i} \oint_{C} \frac{\eta(f(z))}{z-z_{0}} \frac{h-k}{\left(z-z_{0}-h\right)\left(z-z_{0}-k\right)} d z\right| \\
& \leq r \max _{z \in C} \frac{|\eta(f(z))|}{\left|z-z_{0}\right|} \frac{|h-k|}{\left|z-z_{0}-h\right|\left|z-z_{0}-k\right|}
\end{aligned}
$$




$$
\begin{aligned}
& \left.\leq r \frac{4}{r^{2}} \max _{z \in C} \frac{|\eta(f(z))|}{\left|z-z_{0}\right|}|h-k|, \quad \text { by } 5.10\right) \\
& =\frac{4}{r^{2}} \max _{z \in C}|\eta(f(z))||h-k| \\
& \leq \frac{4}{r^{2}}\|\eta\| \max _{z \in C}\|f(z)\||h-k| .
\end{aligned}
$$

Using the fact that $\|x\|=\sup _{\|\eta\|=1}\|\eta(x)\|$ in a Banach space, we obtain

$$
\left|\frac{f\left(z_{0}+h\right)-f\left(z_{0}\right)}{h}-\frac{f\left(z_{0}+k\right)-f\left(z_{0}\right)}{k}\right| \leq \frac{4}{r^{2}} \max _{z \in C}|f(z)||h-k| .
$$

Since $X$ is complete, it follows that the limit

$$
\lim _{h \rightarrow 0} \frac{f\left(z_{0}+h\right)-f\left(z_{0}\right)}{h}
$$

exists. In other words, $a$ is strongly analytic on $I_{\lambda}$.

Conversely, suppose that $a$ is strongly analytic on the strip $I_{\lambda}$. Let $z \in I_{\lambda}$. By hypothesis, there exists $x \in X$ such that

$$
x=\lim _{h \rightarrow 0} \frac{f(z+h)-f(z)}{h} .
$$

Let $\eta \in X^{*}$. Then

$$
\eta(x)=\eta\left(\lim _{h \rightarrow 0} \frac{f(z+h)-f(z)}{h}\right)=\lim _{h \rightarrow 0} \frac{\eta(f(z+h))-\eta(f(z))}{h} .
$$

Hence $\eta \circ f$ is analytic at $z$. Since $z$ and $\eta$ are arbitrary, it follows that $a$ is analytic on $I_{\lambda}$

Corollary 5.1.24. If $\tau=\left\{\tau_{t}\right\}_{t \in \mathbb{R}}$ is a one-parameter $\sigma\left(X, X^{*}\right)$-continuous group of isometries, then $\tau$ is strongly continuous and $X_{\tau}$ is norm-dense in $X$.

Proof. Note that by strongly continuous, we mean that the map $t \mapsto \tau_{t}(a)$ is continuous with respect to the norm in $X$ for every $a \in X$. 
Suppose $X_{\tau}$ is not dense in $X$. Let $H$ be the norm closure of $X_{\tau}$. By hypothesis, $H$ is a proper subspace of $X$. Let $y \in X \backslash H$. By the Hahn-Banach for the sets $H$ and $\{y\}$, there exists a $\varphi \in X^{*}$ such that

$$
\operatorname{Re}(\varphi(y))<\operatorname{Re}(\varphi(y)), \quad h \in H
$$

Since $H$ is a proper subspace of $X$ and $\varphi$ is linear, $\operatorname{Re}(\varphi(H))$ must be either $\{0\}$ or $\mathbb{R}$. By equation (5.11), $\operatorname{Re}(\varphi(H))=\{0\}$.

Note that $\operatorname{Im}(\varphi(H))=0$. In fact, let $h \in H$. Then there is $\lambda \in \mathbb{R}$ such that $\varphi(h)=\lambda i$. Since $H$ is a complex vector space and $\varphi$ is linear, $i h \in H$ and $\varphi(i h)=\lambda$. It follows that $\lambda=0$. The choice of $h$ is arbitrary, thus $\operatorname{Im}(\varphi(H))=\{0\}$. Hence $\varphi$ is zero on $H$ and $\varphi(y) \neq 0$.

From Proposition 5.1.19, $X_{\tau}$ is dense in $X$ with respect to the $\sigma\left(X, X^{*}\right)$-topology. Since $H$ contains $X_{\tau}, H$ is also dense in $X$ in this topology. Thus there exists a sequence $\left\{y_{n}\right\}$ in $H$ converging to $y$ in the $\sigma\left(X, X^{*}\right)$-topology. Since $\varphi \in X^{*}$, we have $\varphi\left(y_{n}\right) \rightarrow \varphi(y)$. This leads to a contradiction because $\varphi(y) \neq 0$ and $\varphi\left(y_{n}\right)=0$ for every $n$. Therefore $H=X$, that is, $X_{\tau}$ is norm dense in $X$.

Now we show that $\tau$ is strongly continuous. Given $a \in X_{\tau}, z \in \mathbb{C}$, it follows from Proposition 5.1 .23 that the limit

$$
\lim _{h \rightarrow 0}\left\|\frac{\tau_{z+h}(a)-\tau_{z}(a)}{h}\right\|
$$

exists. Thus $\left\|\frac{\tau_{z+h}(a)-\tau_{z}(a)}{h}\right\|$ converges to zero as $h \rightarrow 0$, i.e., the function $z \mapsto \tau_{z}(a)$ is continuous with respect to the norm.

Now choose $a \in X$ arbitrary, fix $t \in \mathbb{R}$ and let $\varepsilon>0$. Since $X_{\tau}$ is norm dense in $X$, there exists $a_{\varepsilon} \in X_{\tau}$ such that $\left\|a-a_{\varepsilon}\right\|<\varepsilon / 3$.

Since $a_{\varepsilon} \in X_{\tau}$, there exists $\delta>0$ such that for every $h \in \mathbb{R}$ with $|h|<\delta$, we have 
$\left\|\tau_{t+h}\left(a_{\varepsilon}\right)-\tau_{t}\left(a_{\varepsilon}\right)\right\|<\varepsilon / 3$. Then

$$
\begin{aligned}
\left\|\tau_{t+h}(a)-\tau_{t}(a)\right\| & \leq\left\|\tau_{t+h}(a)-\tau_{t+h}\left(a_{\varepsilon}\right)\right\|+\left\|\tau_{t+h}\left(a_{\varepsilon}\right)-\tau_{t}\left(a_{\varepsilon}\right)\right\|+\left\|\tau_{t}\left(a_{\varepsilon}\right)-\tau_{t}(a)\right\| \\
& \leq\left\|\tau_{t+h}\right\|\left\|a-a_{\varepsilon}\right\|+\left\|\tau_{t+h}\left(a_{\varepsilon}\right)-\tau_{t}\left(a_{\varepsilon}\right)\right\|+\left\|\tau_{t}\right\|\left\|a_{\varepsilon}-a\right\| \\
& =\left\|a-a_{\varepsilon}\right\|+\left\|\tau_{t+h}\left(a_{\varepsilon}\right)-\tau_{t}\left(a_{\varepsilon}\right)\right\|+\left\|a_{\varepsilon}-a\right\| \\
& <\frac{\varepsilon}{3}+\left\|\tau_{t+h}\left(a_{\varepsilon}\right)-\tau_{t}\left(a_{\varepsilon}\right)\right\|+\frac{\varepsilon}{3} \\
& =\frac{2}{3} \varepsilon+\left\|\tau_{t+h}\left(a_{\varepsilon}\right)-\tau_{t}\left(a_{\varepsilon}\right)\right\| \\
& \leq \frac{2}{3} \varepsilon+\frac{\varepsilon}{3} \\
& =\varepsilon .
\end{aligned}
$$

Then the map $t \mapsto \tau_{t}(a)$ is continuous. Since $a$ is arbitrary, $\tau$ is strongly continuous.

Definition 5.1.25. Let $(A, \tau)$ be $\mathrm{C}^{*}$-dynamical system, $\varphi$ a state on $A$ and $\beta \in \mathbb{R}$. We say $\varphi$ is a $\tau$ - $\mathrm{KMS}_{\beta}$-state if

$$
\varphi\left(a \tau_{i \beta}(b)\right)=\varphi(b a)
$$

for all $a, b$ in a $*$-subalgebra $A_{0}$ composed of entire analytic elements such that $A_{0}$ is normdense and $\tau$-invariant.

When $\tau$ is implicit, we just say that $\varphi$ is a $\operatorname{KMS}_{\beta}$-state in order to assert that $\varphi$ is a $\tau$-KMS ${ }_{\beta}$-state. Moreover, if $\beta$ is fixed, we just say $\varphi$ is a KMS state.

Remark 5.1.26. If $\varphi$ is a KMS-state and $\beta=0$, we have

$$
\varphi(a b)=\varphi(b a)
$$

for every $a, b \in A_{0}$. From the continuity of $\varphi$, this equality holds for every $a, b \in A$. In this case, we say the state $\varphi$ is tracial.

Definition 5.1.27. Let $(A, \tau)$ be a $\mathrm{C}^{*}$-dynamical system. We say that $a \in A$ is $\tau$-invariant 
if $\tau_{t}(a)=a$ for every $a \in A$.

Definition 5.1.28. Let $A$ be a $C^{*}$-algebra with dynamics $\tau$. We say that a state $\varphi$ on $A$ is $\tau$-invariant if $\varphi\left(\tau_{t}(a)\right)=\varphi(a)$ for every $a \in A$ and $t \in \mathbb{R}$.

In this section we defined KMS-states as described in [3]. However, Neshveyev's theorem assumes a diferent definition of KMS-states in [17]. The definition used is the item (ii) of the next theorem. Now we show an equivalence in the definition of KMS states when $\beta \neq 0$.

Proposition 5.1.29. Let $A$ be a $C^{*}$-algebra with dynamics given by $\tau$ and let $\beta \in \mathbb{R} \backslash\{0\}$. Given a state $\varphi$ on $A$, the following are equivalent:

(i) $\varphi$ is a $\mathrm{KMS}_{\beta}$-state;

(ii) $\varphi$ is $\tau$-invariant and

$$
\varphi\left(a \tau_{i \beta}(b)\right)=\varphi(b a)
$$

for a dense set of analytic elements $a, b \in A$.

(iii) Equation 5.12 holds for every $a, b \in A_{\tau}$.

Proof. Assume $\beta>0$ without loss of generality.

$(i) \Rightarrow(i i)$ Let $\varphi$ be a $\mathrm{KMS}_{\beta}$-state. By definition, there exists a subalgebra $A_{0}$ composed of entire analytic elements such that $A_{0}$ is norm-dense, $\tau$-invariant and such that 5.12 holds for every $a, b \in A_{0}$. Thus, we only need to show that $\varphi$ is $\tau$-invariant.

Let $a \in A_{0}$. Define $f: \mathbb{C} \rightarrow \mathbb{C}$ by $f(z)=\varphi\left(\tau_{z}(a)\right)$. Since $a$ is entire analytic and $\varphi \in A^{*}$, it follows that $f$ is entire analytic.

Let $\left\{u_{\lambda}\right\}_{\lambda \in \Lambda}$ be an approximate unit for $A$. Fix $\lambda \in \Lambda$. Since $A_{0}$ is norm dense in $A$, for every $\varepsilon>0$ there exists $u_{\lambda}^{(\varepsilon)} \in A_{0}$ satisfying $\left\|u_{\lambda}^{(\varepsilon)}-u_{\lambda}\right\|<\varepsilon$. Both $u_{\lambda}^{(\varepsilon)}, a \in A_{0}$ and $\varphi$ is a $\mathrm{KMS}_{\beta}$-state, then

$$
\varphi\left(u_{\lambda}^{(\varepsilon)} \tau_{i \beta}(a)\right)=\varphi\left(a u_{\lambda}^{(\varepsilon)}\right)
$$


Using the continuity of $\varphi$, we have

$$
\varphi\left(u_{\lambda} \tau_{i \beta}(a)\right)=\varphi\left(a u_{\lambda}\right)
$$

for every $\lambda \in \Lambda$. Again, using the continuity of $\varphi$ and the fact that $\left\{u_{\lambda}\right\}_{\lambda \in \Lambda}$ is an approximate unit, we have

$$
\varphi\left(\tau_{i \beta}(a)\right)=\lim _{\lambda} \varphi\left(u_{\lambda} \tau_{i \beta}(a)\right)=\lim _{\lambda} \varphi\left(a u_{\lambda}\right)=\varphi(a) .
$$

Define $g: \mathbb{C} \rightarrow \mathbb{C}$ by $g(z)=\varphi\left(\tau_{z+i \beta}(a)\right)-\varphi\left(\tau_{z}(a)\right)$. Since $a$ is entire analytic and $\varphi \in A^{*}$, it follows that $g$ is entire analytic. Note that $g$ is zero on the real line. In fact, given $t \in \mathbb{R}$, we have by Lemma 5.1 .21

$$
g(t)=\varphi\left(\tau_{t+i \beta}(a)\right)-\varphi\left(\tau_{t}(a)\right)=\varphi\left(\tau_{i \beta}\left(\tau_{t}(a)\right)\right)-\varphi\left(\tau_{t}(a)\right)=0 .
$$

Hence $g(z)=0$ for every $z \in \mathbb{C}$, that is,

$$
\varphi\left(\tau_{z+i \beta}(a)\right)=\varphi\left(\tau_{z}(a)\right) .
$$

Let $f: \mathbb{C} \rightarrow \mathbb{C}$ be the analytic function defined by $f(z)=\varphi\left(\tau_{z}(a)\right)$. From 5.13 it follows that $f$ is periodic, that is, for every $z \in \mathbb{C}$ there exists $s \in \mathbb{R}$ and $0<t \leq \beta$ such that $f(z)=f(s+i t)$. Then, for every $z \in \mathbb{C}$, we have

$$
\begin{aligned}
|f(z)| & \leq \sup _{w \in \mathbb{C}}|f(w)| \\
& \leq \sup _{\substack{s \in \mathbb{R} \\
0<t \leq \beta}}|f(s+i t)| \\
& =\sup _{\substack{s \in \mathbb{R} \\
0<t \leq \beta}}\left|\varphi\left(\tau_{s+i t}(a)\right)\right| \\
& \leq \sup _{\substack{s \in \mathbb{R} \\
0<t \leq \beta}}\left\|\tau_{s+i t}(a)\right\|
\end{aligned}
$$




$$
\begin{aligned}
& =\sup _{\substack{s \in \mathbb{R} \\
0<t \leq \beta}}\left\|\tau_{s}\left(\tau_{i t}(a)\right)\right\| \text {, by Lemma 5.1.21, } \\
& \leq \sup _{0<t \leq \beta}\left\|\tau_{i t}(a)\right\| \text {, by definition of } \tau_{s} \text { for } s \in \mathbb{R}, \\
& <\infty
\end{aligned}
$$

since $f$ is continuous. Then $f$ is entire analytic and bounded. Therefore $f$ is constant. Thus, for every $z \in \mathbb{C}$, we have

$$
\varphi(a)=f(0)=f(z)=\varphi\left(\tau_{z}(a)\right)
$$

In particular, for every $a \in A_{0}$ and $t \in \mathbb{R}$, we have

$$
\varphi\left(\tau_{t}(a)\right)=\varphi(a)
$$

Since $A_{0}$ is dense in $A$, it follows that (5.14 holds for every $t \in \mathbb{R}, a \in A$. Therefore $\varphi$ is $\tau$-invariant.

$($ ii $) \Rightarrow($ iii $)$ Let $\varphi$ be a $\tau$-invariant state and suppose that there exists a dense set $A_{1}$ of analytic elements such that 5.12 holds for every $a, b \in A_{1}$. Let

$$
\mathcal{D}_{\beta}=\{z \in \mathbb{C}: 0 \leq \operatorname{Im}(z) \leq \beta\}
$$

Let $a, b \in A_{\tau}$. Define $\eta \in A^{*}$ by $\eta(x)=\varphi(a x)$. Then we can define the entire analytic function $f: \mathbb{C} \rightarrow \mathbb{C}$ by $f(z)=\varphi\left(a \tau_{z}(b)\right)$ because $f(z)=\eta\left(\tau_{z}(a)\right)$.

Since $A_{1}$ is dense in $A$, there are sequences $\left\{a_{n}\right\}_{n \in \mathbb{N}},\left\{b_{n}\right\}_{n \in \mathbb{N}}$ in $A_{1}$ such that $a_{n} \rightarrow a$ and $b_{n} \rightarrow b$. Let $a^{\prime}=\sup _{n}\left\|a_{n}\right\|, b^{\prime}=\sup _{n}\left\|b_{n}\right\|$. Both $a^{\prime}, b^{\prime}$ are finite.

For every $n$, there exists $\lambda_{n}>\beta$ such that $b_{n}$ is analytic on the strip $I_{\lambda_{n}}$. So we can define the analytic function $f_{n}$ by $\tilde{f}_{n}(z)=\varphi\left(a_{n} \tau_{z}\left(b_{n}\right)\right)$ for $z \in I_{\lambda_{n}}$. Let $f_{n}=\left.\widetilde{f}_{n}\right|_{\mathcal{D}_{\beta}}$. We will show that $\left\{f_{n}\right\}_{n \in \mathbb{N}}$ is a Cauchy sequence with respect to the supremum norm. 
In fact, given $n, m$, define $\lambda_{n, m}=\min \left\{\lambda_{n}, \lambda_{m}\right\}$. Then $\left.\widetilde{f}_{n}\right|_{I_{\lambda_{n}, m}}-\left.\widetilde{f}_{m}\right|_{I_{\lambda_{n}, m}}$ is analytic on $I_{\lambda_{n, m}}$. Then the supremum of $\left|f_{n}-f_{m}\right|$ is attained on the boundary of $\mathcal{D}_{\beta}$. Hence, for each $z \in \mathcal{D}_{\beta}$, we have

$$
\left|f_{n}(z)-f_{m}(z)\right| \leq \max \left\{\sup _{t \in \mathbb{R}}\left|f_{n}(t)-f_{m}(t)\right|, \sup _{t \in \mathbb{R}}\left|f_{n}(t+i \beta)-f_{m}(t+i \beta)\right|\right\}
$$

However, for every $t \in \mathbb{R}$,

$$
\begin{aligned}
\left|f_{n}(t)-f_{m}(t)\right| & =\left|\varphi\left(a_{n} \tau_{t}\left(b_{n}\right)\right)-\varphi\left(a_{m} \tau_{t}\left(b_{m}\right)\right)\right| \\
& \leq\left|\varphi\left(a_{n} \tau_{t}\left(b_{n}\right)\right)-\varphi\left(a_{n} \tau_{t}\left(b_{m}\right)\right)\right|+\left|\varphi\left(a_{n} \tau_{t}\left(b_{m}\right)\right)-\varphi\left(a_{m} \tau_{t}\left(b_{m}\right)\right)\right| \\
& \leq\left\|a_{n} \tau_{t}\left(b_{n}\right)-a_{n} \tau_{t}\left(b_{m}\right)\right\|+\left\|a_{n} \tau_{t}\left(b_{m}\right)-a_{m} \tau_{t}\left(b_{m}\right)\right\| \\
& \leq\left\|a_{n}\right\|\left\|\tau_{t}\left(b_{n}-b_{m}\right)\right\|+\left\|a_{n}-a_{m}\right\|\left\|\tau_{t}\left(b_{m}\right)\right\| \\
& \leq\left\|a_{n}\right\|\left\|b_{n}-b_{m}\right\|+\left\|a_{n}-a_{m}\right\|\left\|b_{m}\right\| \\
& \leq a^{\prime}\left\|b_{n}-b_{m}\right\|+\left\|a_{n}-a_{m}\right\| b^{\prime} .
\end{aligned}
$$

On the other hand,

$$
\begin{aligned}
\left|f_{n}(t+i \beta)-f_{m}(t+i \beta)\right| & =\left|\varphi\left(a_{n} \tau_{t+i \beta}\left(b_{n}\right)\right)-\varphi\left(a_{m} \tau_{t+i \beta}\left(b_{m}\right)\right)\right| \\
& =\left|\varphi\left(\tau_{t}\left(b_{n}\right) a_{n}\right)-\varphi\left(\tau_{t}\left(b_{m}\right) a_{m}\right)\right| \\
& =\left|\varphi\left(\tau_{t}\left(b_{n}\right) a_{n}\right)-\varphi\left(\tau_{t}\left(b_{n}\right) a_{m}\right)\right|+\left|\varphi\left(\tau_{t}\left(b_{n}\right) a_{m}\right)-\varphi\left(\tau_{t}\left(b_{m}\right) a_{m}\right)\right| \\
& \leq\left\|\tau_{t}\left(b_{n}\right) a_{n}-\tau_{t}\left(b_{n}\right) a_{m}\right\|+\left\|\tau_{t}\left(b_{n}\right) a_{m}-\tau_{t}\left(b_{m}\right) a_{m}\right\| \\
& \leq\left\|\tau_{t}\left(b_{n}\right)\right\|\left\|a_{n}-a_{m}\right\|+\left\|\tau_{t}\left(b_{n}\right)-\tau_{t}\left(b_{m}\right)\right\|\left\|a_{m}\right\| \\
& \leq\left\|b_{n}\right\|\left\|a_{n}-a_{m}\right\|+\left\|b_{n}-b_{m}\right\|\left\|a_{m}\right\| \\
& \leq b^{\prime}\left\|a_{n}-a_{m}\right\|+\left\|b_{n}-b_{m}\right\| a^{\prime} .
\end{aligned}
$$

Therefore $\left\|f_{n}-f_{m}\right\| \leq b^{\prime}\left\|a_{n}-a_{m}\right\|+\left\|b_{n}-b_{m}\right\| a^{\prime}$. Hence $\left\{f_{n}\right\}_{n \in \mathbb{N}}$ is a Cauchy sequence and then this sequence converges to a continuous function $\widetilde{f}: \mathcal{D}_{\beta} \rightarrow \mathbb{C}$ which is analytic on the 
interior of $\mathcal{D}_{\beta}$. Note that for every $t \in \mathbb{R}$,

$$
f(t)=\varphi\left(a \tau_{t}(b)\right)=\lim _{n \rightarrow \infty} \varphi\left(a_{n} \tau_{t}\left(b_{n}\right)\right)=\widetilde{f}(t)
$$

by the continuity of $\varphi$ and $\tau_{t}$. Therefore $\left.f\right|_{\mathcal{D}_{\beta}}=\tilde{f}$ by the uniqueness theorem for analytic functions. Moreover,

$$
\begin{aligned}
\varphi\left(a \tau_{i \beta}(b)\right) & =f(i \beta) \\
& =\lim _{n \rightarrow \infty} f_{n}(i \beta) \\
& =\lim _{n \rightarrow \infty} \varphi\left(a_{n} \tau_{i \beta}\left(b_{n}\right)\right) \\
& =\lim _{n \rightarrow \infty} \varphi\left(b_{n} a_{n}\right) \\
& =\varphi(b a),
\end{aligned}
$$

for every $a, b \in A_{\tau}$. Therefore $\varphi$ is a $\mathrm{KMS}_{\beta}$-state.

$($ iii $) \Rightarrow\left(\right.$ i) From Lemma 5.1.22, $A_{\tau}$ is a $*$-subalgebra and is $\tau$-invariant. By Corollary 5.1.24. $A_{\tau}$ is dense in $A$. Hence $\varphi$ is a $\operatorname{KMS}_{\beta}$-state.

Remark 5.1.30. Let $\varphi$ be a $\tau$-invariant state on a $\mathrm{C}^{*}$-algebra $A$. In order to prove that $\varphi$ is a $\mathrm{KMS}_{\beta}$-state, it suffices to show that $\varphi\left(a \tau_{i \beta}(b)\right)=\varphi(b a)$ in an arbitrary dense $*$-subalgebra of $A$. In fact, if $\beta \neq 0$, this follows directly from Proposition 5.1.29, If $\beta=0$, this follows from the continuity of $\varphi$.

Definition 5.1.31. Given a $C^{*}$-algebra $A$ and a state $\varphi$ on $A$, we define the centralizer of $\varphi$ as the set

$$
\{a \in A: \varphi(a b)=\varphi(b a) \quad \text { for every } b \in A\} .
$$

Lemma 5.1.32. Let $(A, \tau)$ be a $\mathrm{C}^{*}$-dynamical system, $\beta>0$, and $\varphi$ a $\tau$-KMS ${ }_{\beta}$-state. Then, for every $a \in A$ such that $a$ is $\tau$-invariant, it follows that $a$ is in the centralizer of $\varphi$. 
Proof. Let $a \in A$ be $\tau$-invariant. Then $\tau_{t}(a)=a$ for every $t$. Then we can define the function $f: \mathbb{C} \rightarrow A$ by $f(z)=a$. Then we have $f(t)=\tau_{t}(a)$ for every $t \in \mathbb{R}$. The function $f$ is constant, then $f$ is strongly analytic on $\mathbb{C}$. Then, by Proposition 5.1.23, $a$ is entire analytic.

Let $b \in A_{\tau}$. Then,

$$
\varphi(b a)=\varphi\left(b \tau_{i \beta}(a)\right)=\varphi(a b)
$$

because $\varphi$ is a $\tau$ - $\mathrm{KMS}_{\beta}$-state.

Now we show that the set of KMS states, for a fixed $\beta>0$ is convex. Moreover, in order to describe all KMS states on a $\mathrm{C}^{*}$-algebra, it is sufficient to find only its extremal KMS states.

Lemma 5.1.33. Let $A$ be a $C^{*}$-algebra with dynamics $\tau$. Let $\beta \in \mathbb{R}$. The set of $\mathrm{KMS}_{\beta}$-states is convex.

Proof. Let $A$ be a $C^{*}$-algebra with dynamics $\tau$. Fix $\beta \in \mathbb{R}$. Given $\varphi_{1}, \varphi_{2}$ KMS states, $0<t<1$, define $\varphi=t \varphi_{1}+(1-t) \varphi_{2}$. Now we show that $\varphi$ is a KMS state.

(i) $\varphi$ is a state.

Note that $\varphi$ is positive. In fact, given a positive element $a \in A$,

$$
\varphi(a)=t \varphi_{1}(a)+(1-t) \varphi_{2}(a) \geq 0
$$

since both $\varphi_{1}(a), \varphi_{2}(a) \geq 0$. Moreover, we prove that $\varphi$ has norm 1. Applying Lemma 5.1 .2 , we have for every approximate unit $\left\{u_{\lambda}\right\}_{\lambda \in \Lambda}$ of $A$,

$$
\begin{aligned}
\|\varphi\| & =\lim _{\lambda} \varphi\left(u_{\lambda}\right) \\
& =\lim _{\lambda}\left(t \varphi_{1}\left(u_{\lambda}\right)+(1-t) \varphi_{2}\left(u_{\lambda}\right)\right) \\
& =t \lim _{\lambda}\left(\varphi_{1}\left(u_{\lambda}\right)\right)+(1-t) \lim _{\lambda}\left(\varphi_{2}\left(u_{\lambda}\right)\right)
\end{aligned}
$$




$$
\begin{aligned}
& =t\left\|\varphi_{1}\right\|+(1-t)\left\|\varphi_{2}\right\| \\
& =t+(1-t) \\
& =1 .
\end{aligned}
$$

(ii) $\varphi$ is $\mathrm{KMS}$

Given $a, b \in A_{\tau}$,

$$
\varphi\left(a \tau_{i \beta}(b)\right)=t \varphi_{1}\left(a \tau_{i \beta}(b)\right)+(1-t) \varphi_{2}\left(a \tau_{i \beta}(b)\right)=t \varphi_{1}(b a)+(1-t) \varphi_{2}(b a)=\varphi(b a) .
$$

In order to prove that the extremal KMS-states are sufficient to describe all KMS-states, we are going to use a the Krein-Milman theorem. But, before using this theorem, we show that the set of $\mathrm{KMS}_{\beta}$-states is compact with respect to a topology we define below, the weak* topology. Results used in this part can be found on [16] and [22].

Let $X$ be a normed vector space. The weak*-topology on $X^{*}$ is generated by the family of seminorms $p_{x}: X^{*} \rightarrow \mathbb{R}$ such that $\eta \mapsto p_{x}(\eta)=|\eta(x)|$.

Theorem 5.1.34. (The Banach-Alaoglu theorem) If $V$ is a neighborhood of 0 in a normed vector space $X$ and if

$$
K=\left\{\eta \in X^{*}:|\eta(x)| \leq 1 \quad \text { for every } x \in V\right\}
$$

then $K$ is compact in the weak*-topology.

In this case, we can also say that $K$ is weak*-compact.

Lemma 5.1.35. Let $A$ be a $C^{*}$-algebra with dynamics $\tau$. Let $\beta \in \mathbb{R}$. The set of $\mathrm{KMS}_{\beta}$-states is compact in the weak*-topology. 
Proof. Let $\widetilde{A}$ be the unitization of $A$. Let

$$
\begin{aligned}
\widetilde{K} & =\left\{\eta \in \widetilde{A}^{*}:|\eta(a)| \leq 1 \quad \text { for } a \in A \text { with }\|a\| \leq 1\right\} \\
& =\left\{\eta \in \widetilde{A}^{*}:\|\eta\| \leq 1\right\} .
\end{aligned}
$$

It follows from the Banach-Alaoglu theorem that $\widetilde{K}$ is weak*-compact.

Let $\left\{\varphi_{\lambda}\right\}_{\lambda \in \Lambda}$ be a net of $\mathrm{KMS}_{\beta}$-states on $A$. Define for each $\lambda$, $\widetilde{\varphi}_{\lambda}$ the extension of $\varphi_{\lambda}$ on $\widetilde{A}$. Since each $\widetilde{\varphi}_{\lambda} \in \widetilde{K}$ and $\widetilde{K}$ is compact, there exists a subnet $\left\{\widetilde{\varphi}_{\lambda_{j}}\right\}_{j \in J}$ converging to some $\widetilde{\varphi} \in \widetilde{K}$ in the weak*-topology. Hence, for every $a \in A$,

$$
\varphi(a)=\lim _{j \in J} \varphi_{\lambda_{j}}(a)
$$

Also, since each $\widetilde{\varphi}_{\lambda_{j}}$ is a state

$$
\widetilde{\varphi}(1)=\lim _{j \in J} \widetilde{\varphi}_{\lambda_{j}}(1)=1 .
$$

It follows from Theorem 5.1.2 that $\widetilde{\varphi}$ is a state. Hence $\varphi$ is a state. We will prove that $\varphi$ is a $\mathrm{KMS}_{\beta}$-state.

Let $a, b \in A_{\tau}$. Then

$$
\varphi\left(a \tau_{i \beta}(b)\right)=\lim _{j \in J} \varphi_{j}\left(a \tau_{i \beta}(b)\right)=\lim _{j \in J} \varphi_{j}(b a)=\varphi(b a) .
$$

Therefore the set of $\mathrm{KMS}_{\beta}$-states is weak*-compact.

We will use the fact that the set of KMS states is compact and convex to prove that the extremal KMS states are sufficient to describe all KMS states. Before proving this, we define some concepts about convex sets and state then state the Krein-Milman theorem. Here all results are defined for subsets of $X^{*}$. For more general results, see [16].

Definition 5.1.36. Let $X$ be a normed vector space. Given a subset $S$ of $X^{*}, \overline{c o}(S)$ denotes the closure of $\operatorname{co}(S)$ with respect to the weak*-topology. 
Now we state the Krein-Milman theorem for $X^{*}$.

Theorem 5.1.37. Let $X$ be a normed vector space. Let $C \subset X^{*}$ be a non-empty convex weak*-compact subset. Then the set $E$ of extreme points of $C$ is non-empty and

$$
C=\overline{\operatorname{co}}(E)
$$

Moreover, if $S$ is a closed subset of $C$ with respect to the weak*-topology such that $\overline{\operatorname{co}}(S)=C$, then $S$ contains $E$.

Now we apply the Krein-Milman theorem to show that the extremal KMS states are sufficient to describe all KMS states on $A$.

Corollary 5.1.38. Let $A$ be a $C^{*}$-algebra and $\tau$ its dynamics. Fix $\beta>0$. Let $C$ denote the set of $\mathrm{KMS}_{\beta^{-}}$-states on $A$ and assume $C \neq \varnothing$. Let $E$ be the set of extremal $\mathrm{KMS}_{\beta^{-}}$-states. Then $C=\overline{\operatorname{co}}(E)$ and $E \neq \varnothing$.

Proof. It follows from Lemmas 5.1.33 and 5.1.35 that $C$ is convex and compact. By hypothesis, $C \neq \varnothing$. Then we can apply the Krein-Milman theorem and the result follows.

\subsection{First Theorem}

Let $G$ be a locally compact Hausdorff second countable étale groupoid. Neshveyev's first theorem shows that for every state $\varphi$ on $C^{*}(G)$ with centralizer containing $C_{0}\left(G^{(0)}\right)$ there is a corresponding pair $\left(\mu,\left\{\varphi_{x}\right\}_{x}\right)$, where $\mu$ is a probability measure on $G^{(0)}$, and $\left\{\varphi_{x}\right\}_{x}$ is a $\mu$-measurable field of states $\varphi_{x}$ on $C^{*}\left(G_{x}^{x}\right)$ for $x \in G^{(0)}$. The results in this section can be found in [17].

In Section 5.3, we will define a dynamics $\tau$ on $C^{*}(G)$ such that every function in $C_{0}\left(G^{(0)}\right)$ is $\tau$-invariant. Thus, by Lemma 5.1 .32 , the centralizer of every KMS state contains $C_{0}\left(G^{(0)}\right)$. Therefore, we can apply Neshveyev's first theorem to KMS states.

Now we describe $\mu$-measurable field of states as described in [5]. 
Definition 5.2.1. Let $G$ be a locally compact Hausdorff second countable étale groupoid, and let $\mu$ be a Radon Borel measure on $G^{(0)}$. For each $x \in G^{(0)}, g \in G_{x}^{x}$, we let $u_{g}$ denote the canonical unitary generators of $C^{*}\left(G_{x}^{x}\right)$, i.e., $u_{g} \in C_{c}\left(G_{x}^{x}\right)$ is a function defined by $u_{g}(g)=1$ and $u_{g}(h)=0$ if $h \neq g$.

We call a collection $\left\{\varphi_{x}\right\}_{x \in G^{(0)}}$ a $\mu$-measurable field of states if each $\varphi_{x}$ is a state on $C^{*}\left(G_{x}^{x}\right)$ and the function:

$$
G^{(0)} \ni x \mapsto \sum_{g \in G_{x}^{x}} f(g) \varphi_{x}\left(u_{g}\right)
$$

is $\mu$-measurable for each $f \in C_{c}(G)$.

Remark 5.2.2. Fix a probability measure $\mu$ on $G^{(0)}$. Given a conull set $V \subset G^{(0)}$, let $\left\{\varphi_{x}\right\}_{x \in V}$ be a family of states $\varphi_{x}$ on $C^{*}\left(G_{x}^{x}\right)$ such that for every $f \in C_{c}(G)$ the function

$$
x \mapsto \chi_{V}(x) \sum_{g \in G_{x}^{x}} f(g) \varphi_{x}\left(u_{g}\right)
$$

is $\mu$-measurable. Then there exists a $\mu$-measurable field of states $\left\{\widetilde{\varphi}_{x}\right\}_{x \in G^{(0)}}$ such that $\widetilde{\varphi}_{x}=$ $\varphi_{x}$ for every $x \in V$. In fact, any $\mathrm{C}^{*}$-algebra $C^{*}\left(G_{x}^{x}\right)$ has at least on state, then you can just choose any family of states $\widetilde{\varphi}_{x}$ on $C^{*}\left(G_{x}^{x}\right)$ such that $\widetilde{\varphi}_{x}=\varphi_{x}$ for $x \in V$. Since the function defined by $(5.15)$ is $\mu$-measurable, then $\left\{\widetilde{\varphi}_{x}\right\}$ is a $\mu$-measurable field of states.

Later we will prove in Neshveyev's first theorem that if $\left\{\varphi_{x}\right\}_{x \in G^{(0)}},\left\{\widetilde{\varphi}_{x}\right\}_{x \in G^{(0)}}$ are two $\mu$-measurable field of states whose states are equal on a conull subset of $G^{(0)}$, then $\left(\mu,\left\{\varphi_{x}\right\}_{x}\right)$ and $\left(\mu,\left\{\widetilde{\varphi}_{x}\right\}_{x}\right)$ define the same state.

Remark 5.2.3. When there is no risk of confusion, we denote the $\mu$-measurable field of states $\left\{\varphi_{x}\right\}_{x \in G^{(0)}}$ by $\left\{\varphi_{x}\right\}_{x}$.

Lemma 5.2.4. Let $G$ be a locally compact Hausdorff second countable étale groupoid and let $x \in G^{(0)}$. Given $g_{1}, g_{2}, g \in G_{x}^{x}, u_{g_{1}} \cdot u_{g_{2}}=u_{g_{1} g_{2}}$ and $u_{g}{ }^{*}=u_{g^{-1}}$. 
Proof. Let $h \in G_{x}^{x}$. Then

$$
u_{g_{1}} \cdot u_{g_{2}}(h)=\sum_{h_{1} h_{2}} u_{g_{1}}\left(h_{1}\right) u_{g_{2}}\left(h_{2}\right) .
$$

Note that for every $h_{1}, h_{2}$ such that $h_{1} \neq g_{1}$ or $h_{2} \neq g_{2}$, we have $u_{g_{1}} \cdot u_{g_{2}}(h)=0$. Thus,

$$
u_{g_{1}} \cdot u_{g_{2}}(h)=\left\{\begin{array}{ll}
1 & \text { if } h=g_{1} g_{2}, \\
0 & \text { otherwise }
\end{array}=u_{g_{1} g_{2}}(h) .\right.
$$

We also have

$$
\begin{aligned}
u_{g}^{*}(h) & =\overline{u_{g}\left(h^{-1}\right)} \\
& =u_{g}\left(h^{-1}\right), \quad \text { since } u_{g} \text { is real-valued, } \\
& = \begin{cases}1 & \text { if } h^{-1}=g \\
0 & \text { otherwise }\end{cases} \\
& = \begin{cases}1 & \text { if } h=g^{-1} \\
0 & \text { otherwise }\end{cases} \\
& =u_{g^{-1}}(h)
\end{aligned}
$$

Therefore the result follows.

Remark 5.2.5. If $\mu$ is purely atomic, it follows from Lemma 2.5 .2 that that every family $\left\{\varphi_{x}\right\}_{x \in G^{(0)}}$ of states $\varphi_{x}$ on $C^{*}\left(G_{x}^{x}\right)$ is a $\mu$-measurable field of states.

Given a state $\varphi$ on $C^{*}(G)$, we obtain from Renault's disintegration theorem a unitary representation of $G$. The following lemma uses this representation to find a representation $\pi_{x}$ on $C^{*}\left(G_{x}^{x}\right)$ for each $x \in G^{(0)}$. In the proof of Neshveyev's first theorem, we will use $\pi_{x}$ to define a state $\varphi_{x}$ on $C^{*}\left(G_{x}^{x}\right)$. 
Lemma 5.2.6. Let $G$ be a locally compact Hausdorff second countable étale groupoid. Let $\left(\mu, G^{(0)} * \mathfrak{H}, L\right)$ be a unitary representation of $G$. Let $x \in G^{(0)}$. The linear map $\pi_{x}: C^{*}\left(G_{x}^{x}\right) \rightarrow$ $B\left(\mathcal{H}_{x}\right)$ defined by $\pi_{x}\left(u_{g}\right)=L_{g}$ is a representation of $C^{*}\left(G_{x}^{x}\right)$.

Proof. Let $x \in G^{(0)}$. Let $g \in G_{x}^{x}$, then

$$
\pi_{x}\left(u_{g}^{*}\right)=\pi_{x}\left(u_{g^{-1}}\right)=L_{g^{-1}}=L_{g}{ }^{*}=\pi_{x}\left(u_{g}\right)^{*}
$$

Given $g_{1}, g_{2} \in G_{x}^{x}$,

$$
\pi_{x}\left(u_{g_{1}} u_{g_{2}}\right)=\pi_{x}\left(u_{g_{1} g_{2}}\right)=L_{g_{1} g_{2}}=L_{g_{1}} L_{g_{2}}=\pi_{x}\left(u_{g_{1}}\right) \pi_{x}\left(u_{g_{2}}\right)
$$

Given a state $\varphi_{x}$ on $C^{*}\left(G_{x}^{x}\right)$, it corresponds to a representation $\pi_{x}: C^{*}\left(G_{x}^{x}\right) \rightarrow B\left(\mathcal{H}_{x}\right)$. We can use $\pi_{x}$ to define a unitary map $L_{g}: \mathcal{H}_{x} \rightarrow \mathcal{H}_{x}$ by $L_{g}=\pi_{x}\left(u_{g}\right)$, for every $g \in G_{x}^{x}$. Using this fact, we can apply Lemma 5.2.7 and obtain a Hilbert space $K_{x}$. We use this result in the proof of Neshveyev's first theorem to show that there exists a state $\psi_{x}$ corresponding to $K_{x}$. Then we use Lemma 5.1 .8 to find the state $\varphi$ on $C^{*}(G)$.

Lemma 5.2.7. Let $G$ be a locally compact Hausdorff second countable étale groupoid with Haar system defined by the counting measures on $G^{x}$. Let $\left\{L_{h}\right\}_{h \in G^{\prime}}$ be a family of unitary operators $L_{h}: \mathcal{H}_{x} \rightarrow \mathcal{H}_{x}$ on a Hilbert space $\mathcal{H}_{x}$ defined for all $h \in G_{x}^{x}$ and $x \in G^{(0)}$. Given $x \in G^{(0)}$, let $K_{x}$ be the family of functions $v: G_{x} \rightarrow \mathcal{H}_{x}$ such that

$$
v(g h)=L_{h}^{*} v(g) \quad \text { for } g \in G_{x} \text { and } h \in G_{x}^{x}
$$

and

$$
\sum_{g \in G_{x} / G_{x}^{x}}\|v(g)\|^{2}<\infty
$$


Then $K_{x}$ is a Hilbert space.

Proof. Note that $G_{x} / G_{x}^{x}$ is defined by the following equivalence relation on $G_{x}$ :

$$
g_{1} \sim g_{2} \text { if } g_{1}=g_{2} h \text { for some } h \in G_{x}^{x}
$$

In order to prove that (5.17) is well-defined, we will show that $\left\|v\left(g_{1}\right)\right\|=\left\|v\left(g_{2}\right)\right\|$ in this case. In fact, by definition of $v$,

$$
\left\|v\left(g_{1}\right)\right\|=\left\|v\left(g_{2} h\right)\right\|=\left\|L_{h}^{*} v\left(g_{2}\right)\right\|=\left\|v\left(g_{2}\right)\right\|
$$

since $L_{h}$ is unitary.

$K_{x}$ is a vector space. In fact, let $u, v \in K_{x}, \lambda \in \mathbb{C}$. Then, for $g \in G_{x}, h \in G_{x}^{x}$,

$$
\begin{aligned}
(u+\lambda v)(g h) & =u(g h)+\lambda v(g h) \\
& =L_{h}{ }^{*} u(g)+L_{h}{ }^{*} \lambda v(g) \\
& =L_{h}{ }^{*}[(u+\lambda v)(g)] .
\end{aligned}
$$

Now we can define the inner product on $K_{x}$ by

$$
\langle u, v\rangle=\sum_{g \in G_{x} / G_{x}^{x}}\langle u(g), v(g)\rangle .
$$

Note that (5.18) defines an inner product on $K_{x}$. In fact, given $g_{1}, g_{2} \in G_{x}, h \in G_{x}^{x}$ such that $g_{1}=g_{2} h$, we have for $u, v \in K_{x}$,

$$
\left\langle u\left(g_{1}\right), v\left(g_{1}\right)\right\rangle=\left\langle u\left(g_{2} h\right), v\left(g_{2} h\right)\right\rangle=\left\langle L_{h}{ }^{*} u\left(g_{2}\right), L_{h}{ }^{*} v\left(g_{2}\right)\right\rangle=\left\langle u\left(g_{2}\right), v\left(g_{2}\right)\right\rangle .
$$

It is easy to show this operation satisfies

$$
\left\langle u_{1}+\lambda u_{2}, v\right\rangle=\left\langle u_{1}, v\right\rangle+\lambda\left\langle u_{2}, v\right\rangle, \quad\langle u, u\rangle \geq 0 \quad \text { and } \quad\langle u, v\rangle=\overline{\langle v, u\rangle}
$$


for $u, u_{1}, u_{2}, v \in K_{x}, \lambda \in \mathbb{C}$.

Suppose $\langle v, v\rangle=0$. Let $g \in G_{x}$, then $\langle v(g), v(g)\rangle=0$ by definition. Therefore $v=0$. We denote the norm defined in (5.17) by $\|\cdot\|$.

Now we prove that $K_{x}$ is Banach. Let $\left\{v_{n}\right\}$ be a Cauchy sequence on $K_{x}$. Given $g \in G_{x}$, $\left\{v_{n}(g)\right\}$ is a Cauchy sequence on $\mathcal{H}_{x}$, hence $v_{n}(g) \rightarrow v(g)$, where $v: G_{x} \rightarrow \mathcal{H}_{x}$ is a function.

We show that $v$ satisfies (5.16). Let $g \in G_{x}, h \in G_{x}^{x}$. Then $v_{n}(g h) \rightarrow v(g h)$. But for each $n, v_{n}(g h)=L_{h}{ }^{*} v_{n}(g)$. Since $L_{h}$ is unitary, it follows that

$$
v(g h)=\lim _{n \rightarrow \infty} v_{n}(g h)=\lim _{n \rightarrow \infty} L_{h}{ }^{*} v_{n}(g)=L_{h}{ }^{*} v(g) .
$$

Now we prove that $v_{n}$ converges to $v$ with respect to the norm. Since $G$ is second countable and étale, it follows from Proposition 3.2.11 that $G_{x}$ is countable. Then $G_{x} / G_{x}^{x}$ is countable. If $G_{x} / G_{x}^{x}$ is finite, then pointwise convergence implies convergence in the norm. So we assume $G_{x} / G_{x}^{x}$ is infinite and denote its elements by a sequence $\left\{g_{k}\right\}$. Given $\varepsilon>0$, let $n_{0} \geq 0$ such that for every $n, m \geq n_{0}$, we have $\left\|v_{n}-v_{m}\right\|<\varepsilon$.

Fix $k_{1}$. If $n \geq n_{0}$, we have

$$
\sum_{k=1}^{k_{1}}\left\|v_{n}\left(g_{k}\right)-v\left(g_{k}\right)\right\|^{2}=\lim _{m \rightarrow \infty} \sum_{k=1}^{k_{1}}\left\|v_{n}\left(g_{k}\right)-v_{m}\left(g_{k}\right)\right\|^{2} \leq \varepsilon^{2} .
$$

Since $k_{1}$ is arbitrary, we have

$$
\left\|v_{n}-v\right\|^{2}=\lim _{k_{1} \rightarrow \infty} \sum_{k=1}^{k_{1}}\left\|v_{n}\left(g_{k}\right)-v\left(g_{k}\right)\right\|^{2} \leq \varepsilon^{2} .
$$

Hence $\left\|v_{n}-v\right\| \rightarrow 0$. Therefore $v_{n}$ converges to $v$ in the norm. Moreover, it is easy to see that $\|v\|<\infty$. In fact, choose $n$ such that $\left\|v_{n}-v\right\|<1$. Hence $\|v\| \leq\left\|v_{n}-v\right\|+\left\|v_{n}\right\|<$ $1+\left\|v_{n}\right\|<\infty$.

Proposition 5.2.8. Let $G$ be a locally compact Hausdorff second countable étale groupoid. Let $f \in C_{c}(G)$ and assume there exists an open set $U$ including the support of $f$ such that 
$U \cap G^{\prime}=\varnothing$. Then we can write $f=f_{1}+\ldots+f_{n}$ where for every $i=1, \ldots, n, f_{i} \in C_{c}\left(\mathcal{U}_{i}\right)$ and $\mathcal{U}_{i}$ is an open bisection satisfying $r\left(\mathcal{U}_{i}\right) \cap s\left(\mathcal{U}_{i}\right)=\varnothing$.

Proof. Let $f \in C_{c}(G)$ and $U$ an open set $U$ containing its support such that $U \cap G^{\prime}=\varnothing$. Since $K$ is compact, there exists an open set $V$ whose closure is compact and $K \subset \bar{V} \subset U$. Then, by Lemma 3.2.13, for every $g \in V$ there exists an open bisection $\mathcal{U}_{g}$ containing $g$ such that $r\left(\mathcal{U}_{g}\right) \cap s\left(\mathcal{U}_{g}\right)=\varnothing$. However, $\bar{V}$ is compact, then there are $g_{1}, \ldots, g_{n}$ such that $\mathcal{U}_{g_{1}}, \ldots, \mathcal{U}_{g_{n}}$ cover $V$. Denote $\mathcal{U}_{i}=\mathcal{U}_{g_{i}}$ for $i=1, \ldots, n$.

Let $h_{i} i=1^{n}$ be the partition of unity subordinate to the open cover $\left\{\mathcal{U}_{i}\right\}_{i=1}^{n}$. For each $i=1, \ldots, n$, let $f_{i}=h_{i} f$. Then $f_{i} \in C_{c}\left(\mathcal{U}_{i}\right)$ and $f=f_{1}+\ldots+f_{n}$.

Theorem 5.2.9. (Neshveyev) Let $G$ be a locally compact Hausdorff second countable étale groupoid. There is a one-to-one correspondence between states on $C^{*}(G)$ with centralizer containing $C_{0}\left(G^{(0)}\right)$ and pairs $\left(\mu,\left\{\varphi_{x}\right\}_{x}\right)$ consisting of a probability measure $\mu$ on $G^{(0)}$ and a $\mu$-measurable field of states $\varphi_{x}$ on $C^{*}\left(G_{x}^{x}\right)$. Namely, the state corresponding to $\left(\mu,\left\{\varphi_{x}\right\}_{x}\right)$ is given by

$$
\varphi(f)=\int_{G^{(0)}} \sum_{g \in G_{x}^{x}} f(g) \varphi_{x}\left(u_{g}\right) d \mu(x) \quad \text { for } \quad f \in C_{c}(G)
$$

Proof. Endow $G$ with the Haar system given by counting measures $\lambda^{x}$ on $G^{x}$.

Assume $\varphi$ is a state on $C^{*}(G)$ with centralizer containing $C_{0}\left(G^{(0)}\right)$. Let $(\mathcal{H}, \pi, \xi)$ be the corresponding GNS-triple. It follows from Lemma 4.3.5 that $\pi$ satisfies the conditions of Renault's disintegration theorem. Therefore there is a unitary representation $\left(\mu, G^{(0)} * \mathfrak{H}, L\right)$ of $G$ such that $\mathcal{H}$ is isomorphic to $L^{2}\left(G^{(0)} * \mathfrak{H}, \mu\right)$ and $\pi$ is equivalent to the integrated form of $\left(\mu, G^{(0)} * \mathfrak{H}, L\right)$. Here we identify $\mathcal{H}$ with $L^{2}\left(G^{(0)} * \mathfrak{H}, \mu\right)$ without loss of generality. Hence,

$$
\begin{aligned}
\varphi(f) & =\langle\pi(f) \xi, \xi\rangle \\
& =\int_{G} f(g)\left\langle L_{g} \xi_{s(g)}, \xi_{r(g)}\right\rangle_{\mathcal{H}_{r(g)}} \Delta(g)^{-\frac{1}{2}} d \mu_{r}(g)
\end{aligned}
$$




$$
=\int_{G^{(0)}} \sum_{g \in G^{x}} f(g)\left\langle L_{g} \xi_{s(g)}, \xi_{x}\right\rangle_{\mathcal{H}_{x}} \Delta(g)^{-\frac{1}{2}} d \mu(x) .
$$

Now we prove that for every $f \in C_{c}(G)$ and for $\mu$-a.e. $x \in G^{(0)}$ we have

$$
\sum_{g \in G^{x} \backslash G_{x}^{x}} f(g)\left\langle L_{g} \xi_{s(g)}, \xi_{x}\right\rangle_{\mathcal{H}_{x}} \Delta(g)^{-\frac{1}{2}}=0 .
$$

Using the lineariy of 5.20 with respect to $f$ we can assume it is supported on an open bisection $\mathcal{U}$. Since the isotropy bundle $G^{\prime}$ is closed from Lemma 3.2 .13 and the sum in $(5.20)$ does not take into account elements in $G^{\prime}$, we can assume $\mathcal{U} \cap G^{\prime}=\varnothing$. Then, by Proposition 5.2.8, we can assume $r(\mathcal{U}) \cap s(\mathcal{U})=\varnothing$ without loss of generality.

Let $h \in C_{c}(r(\mathcal{U}))$. Then $f \cdot h=0$. In fact, given $g \in G,(f \cdot h)(g)=f(g) h(s(g))$. Suppose $f(g) \neq 0$. Then $g \in \mathcal{U}$. Thus $s(g) \in s(\mathcal{U})$. Since $s(\mathcal{U}) \cap r(\mathcal{U})=\varnothing$ by hypothesis, it follows that $s(g) \notin r(\mathcal{U})$. Thus $h(s(g))=0$. Therefore $f \cdot h=0$.

Since $\varphi$ has centralizer containing $C_{0}\left(G^{(0)}\right)$, we have $\varphi(h \cdot f)=\varphi(f \cdot h)=0$. Applying 5.19) for $h \cdot f$, it follows that

$$
\begin{aligned}
0 & =\varphi(h \cdot f) \\
& =\int_{G^{(0)}} \sum_{g \in G^{x}}(h \cdot f)(g)\left\langle L_{g} \xi_{s(g)}, \xi_{x}\right\rangle \Delta(g)^{-\frac{1}{2}} d \mu(x) \\
& =\int_{G^{(0)}} \sum_{g \in G^{x}} h(r(g)) f(g)\left\langle L_{g} \xi_{s(g)}, \xi_{x}\right\rangle \Delta(g)^{-\frac{1}{2}} d \mu(x), \quad \text { by Lemma 3.3.4. } \\
& =\int_{G^{(0)}} h(x) \sum_{g \in G^{x}} f(g)\left\langle L_{g} \xi_{s(g)}, \xi_{x}\right\rangle \Delta(g)^{-\frac{1}{2}} d \mu(x) \\
& =\int_{r(\mathcal{U})} h(x) \sum_{g \in G^{x}} f(g)\left\langle L_{g} \xi_{s(g)}, \xi_{x}\right\rangle \Delta(g)^{-\frac{1}{2}} d \mu(x), \text { since } h \in C_{c}(r(\mathcal{U})), \\
& =\int_{r(\mathcal{U})} h(x) \sum_{g \in G^{x} \backslash G_{x}^{x}} f(g)\left\langle L_{g} \xi_{s(g)}, \xi_{x}\right\rangle \Delta(g)^{-\frac{1}{2}} d \mu(x), \text { since } \mathcal{U} \cap G^{\prime}=\varnothing .
\end{aligned}
$$

Since $h \in C_{c}(r(\mathcal{U}))$ is arbitrary, it follows that for $\mu$-a.e $x \in r(\mathcal{U})$, 5.20 holds. Since 
$f \in C_{c}(\mathcal{U}), 5.20$ holds for every $x \notin r(\mathcal{U})$. Indeed, given $x \notin r(\mathcal{U}), g \in G^{x}$, then $g \notin \mathcal{U}$. Hence $f(g)=0$. Therefore $(5.20)$ holds for all $f \in C_{c}(\mathcal{U})$, for $\mu$-a.e. $x$. Therefore, (5.20) is valid for every $f \in C_{c}(G)$.

Let $f \in C_{c}(G)$. Applying 5.20 on 5.19 , we have

$$
\begin{aligned}
\varphi(f) & =\int_{G^{(0)}} \sum_{g \in G^{x}} f(g)\left\langle L_{g} \xi_{s(g)}, \xi_{x}\right\rangle_{\mathcal{H}_{x}} \Delta(g)^{-\frac{1}{2}} d \mu(x) \\
& =\int_{G^{(0)}} \sum_{g \in G_{x}^{x}} f(g)\left\langle L_{g} \xi_{s(g)}, \xi_{x}\right\rangle_{\mathcal{H}_{x}} \Delta(g)^{-\frac{1}{2}} d \mu(x) \\
& =\int_{G^{(0)}} \sum_{g \in G_{x}^{x}} f(g)\left\langle L_{g} \xi_{x}, \xi_{x}\right\rangle_{\mathcal{H}_{x}} \Delta(g)^{-\frac{1}{2}} d \mu(x) .
\end{aligned}
$$

From Lemma 5.2.6, we can define for every $x \in G^{(0)}$ a representation $\pi_{x}: C^{*}\left(G_{x}^{x}\right) \rightarrow$ $B\left(\mathcal{H}_{x}\right)$ such that $\pi_{x}\left(u_{g}\right)=L_{g}$ for all $g \in G_{x}^{x}$. Then

$$
\varphi(f)=\int_{G^{(0)}} \sum_{g \in G_{x}^{x}} f(g)\left\langle\pi_{x}\left(u_{g}\right) \xi_{x}, \xi_{x}\right\rangle_{\mathcal{H}_{x}} \Delta(g)^{-\frac{1}{2}} d \mu(x)
$$

By Proposition 4.1.8, $\Delta(g)=1$ for all $g \in G_{x}^{x}$ for $\mu$-a.e. $x \in G^{(0)}$. Hence,

$$
\varphi(f)=\int_{G^{(0)}} \sum_{g \in G_{x}^{x}} f(g)\left\langle\pi_{x}\left(u_{g}\right) \xi_{x}, \xi_{x}\right\rangle_{\mathcal{H}_{x}} d \mu(x) .
$$

Since $\xi \in L^{2}(X * \mathfrak{H}, \mu)$, the set $V \subset G^{(0)}$ of elements $x$ such that $\xi_{x} \neq 0$, is measurable. Let $\widetilde{\mu}$ be a measure on $G^{(0)}$ such that $d \widetilde{\mu} / d \mu(x)=\left\|\xi_{x}\right\|^{2}$. Note that $\widetilde{\mu}$ is supported on $V$. Moreover, $\widetilde{\mu}$ is a probability measure. In fact,

$$
\int_{G^{(0)}} d \widetilde{\mu}(x)=\int_{G^{(0)}}\left\|\xi_{x}\right\|^{2} d \mu(x)=\|\xi\|^{2}=1
$$

Let $\widetilde{\xi}$ be defined by $\widetilde{\xi}_{x}=0$ if $x \notin V$, and $\widetilde{\xi}_{x}=\xi_{x} /\left\|\xi_{x}\right\|$ if $x \in V$. Then $\widetilde{\xi} \in L^{2}(X * \mathfrak{H}, \mu)$ and 
$\|\widetilde{\xi}\|=1$. Moreover,

$$
\begin{aligned}
\varphi(f) & =\int_{G^{(0)}} \sum_{g \in G_{x}^{x}} f(g)\left\langle\pi_{x}\left(u_{g}\right) \xi_{x}, \xi_{x}\right\rangle_{\mathcal{H}_{x}} d \mu(x) \\
& =\int_{V} \sum_{g \in G_{x}^{x}} f(g)\left\langle\pi_{x}\left(u_{g}\right) \xi_{x}, \xi_{x}\right\rangle_{\mathcal{H}_{x}} d \mu(x) \\
& =\int_{V} \sum_{g \in G_{x}^{x}} f(g) \frac{\left\langle\pi_{x}\left(u_{g}\right) \xi_{x}, \xi_{x}\right\rangle_{\mathcal{H}_{x}}}{\left\|\xi_{x}\right\|^{2}}\left\|\xi_{x}\right\|^{2} d \mu(x) \\
& =\int_{V} \sum_{g \in G_{x}^{x}} f(g)\left\langle\pi_{x}\left(u_{g}\right) \widetilde{\xi}_{x}, \widetilde{\xi}_{x}\right\rangle_{\mathcal{H}_{x}}\left\|\xi_{x}\right\|^{2} d \mu(x) \\
& =\int_{V} \sum_{g \in G_{x}^{x}} f(g)\left\langle\pi_{x}\left(u_{g}\right) \widetilde{\xi}_{x}, \widetilde{\xi}_{x}\right\rangle_{\mathcal{H}_{x}} d \widetilde{\mu}(x)
\end{aligned}
$$

For every $x \in V$, there is a state $\varphi_{x}$ on $C^{*}\left(G_{x}^{x}\right)$ such that

$$
\varphi_{x}\left(u_{g}\right)=\left\langle\pi_{x}\left(u_{g}\right) \widetilde{\xi}_{x}, \widetilde{\xi}_{x}\right\rangle_{\mathcal{H}_{x}}, \quad \text { for every } g \in G_{x}^{x}
$$

From Remark 5.2 .2 , we can choose a $\widetilde{\mu}$-measurable field of states $\left\{\varphi_{x}\right\}$ such that $\varphi_{x}$ is defined by (5.21) for every $x \in V$. Therefore,

$$
\begin{aligned}
\varphi(f) & =\int_{V} \sum_{g \in G_{x}^{x}} f(g) \varphi_{x}\left(u_{g}\right) d \widetilde{\mu}(x) \\
& =\int_{G^{(0)}} \sum_{g \in G_{x}^{x}} f(g) \varphi_{x}\left(u_{g}\right) d \widetilde{\mu}(x) .
\end{aligned}
$$

Conversely, let $\mu$ be a probability measure on $G^{(0)}$ and let a $\mu$-measurable field of states $\varphi_{x}$ on $C^{*}\left(G_{x}^{x}\right)$.

Given $x$, let $\left(\mathcal{H}_{x}, \pi_{x}, \xi_{x}\right)$ be the GNS-triple for $\varphi_{x}$. Define for every $h \in G_{x}^{x}, L_{h}=\pi_{x}\left(u_{h}\right)$. Each $L_{h}$ is unitary, since

$$
L_{h}^{*}=\pi_{x}\left(u_{h}\right)^{*}=\pi_{x}\left(u_{h}^{*}\right)=\pi_{x}\left(u_{h^{-1}}\right)=\pi_{x}\left(u_{h}^{-1}\right)=\pi_{x}\left(u_{h}\right)^{-1}=L_{h}^{-1} .
$$


Let $K_{x}$ be the Hilbert space as in Lemma 5.2.7. Let $\vartheta_{x}: C_{c}(G) \rightarrow B\left(K_{x}\right)$ be defined by

$$
\left(\vartheta_{x}(f) v\right)(g)=\sum_{h \in G^{r(g)}} f(h) v\left(h^{-1} g\right) \text { for } f \in C_{c}(G), g \in G_{x}
$$

First we show that each $\vartheta_{x}(f)$ is in $B\left(K_{x}\right)$. In fact, $\vartheta_{x}(f)$ is linear on $K_{x}$ by definition. Let $v \in K_{x}, g \in G_{x}$ and $k \in G_{x}^{x}$. Then

$$
\begin{aligned}
\left(\vartheta_{x}(f) v\right)(g k) & =\sum_{h \in G^{r(g)}} f(h) v\left(h^{-1} g k\right) \\
& =\sum_{h \in G^{r(g)}} f(h) L_{k}^{*} v\left(h^{-1} g\right) \\
& =L_{k}^{*}\left(\sum_{h \in G^{r(g)}} f(h) v\left(h^{-1} g\right)\right) \\
& =L_{k} *\left[\left(\vartheta_{x}(f) v\right)(g)\right] .
\end{aligned}
$$

Also, we need to show that $\left\|\vartheta_{x}(f)\right\|^{2}<\infty$ for every $f \in C_{c}(G)$. Since $\vartheta_{x}$ is linear, we can assume $f \in C_{c}(\mathcal{U})$, where $\mathcal{U} \subset G$ is an open bisection.

Let $g \in G_{x}$. Assume $r(g) \notin r(\mathcal{U})$. Then, for every $h \in G^{r(g)}$, it follows that $h \notin \mathcal{U}$, which implies $f(h)=0$. Hence $\left(\vartheta_{x}(f) v\right)(g)=0$. Now assume $r(g) \in r(\mathcal{U})$. Since $\mathcal{U}$ is an open bisection, there exists a unique $h^{r(g)}$ in $\mathcal{U}$ such that $h^{r(g)} \in G^{r(g)}$. Then

$$
\left(\vartheta_{x}(f) v\right)(g)=\sum_{h \in G^{r(g)}} f(h) v\left(h^{-1} g\right)=f\left(h^{r(g)}\right) v\left(\left(h^{r(g)}\right)^{-1} g\right) .
$$

Note that if $g_{1}, g_{2} \in G_{x}$ are equivalent, then $r\left(g_{1}\right)=r\left(g_{2}\right)$. Indeed, there is $k \in G_{x}^{x}$ such that $g_{1}=g_{2} k$. Hence $r\left(g_{1}\right)=r\left(g_{2} k\right)=r\left(g_{2}\right)$. Moreover, if $r\left(g_{1}\right) \in r(\mathcal{U})$, it follows that

$$
\begin{aligned}
\left\|v\left(\left(h^{r\left(g_{1}\right)}\right)^{-1} g_{1}\right)\right\| & =\left\|v\left(\left(h^{r\left(g_{1}\right)}\right)^{-1} g_{2} k\right)\right\| \\
& =\left\|v\left(\left(h^{r\left(g_{2}\right)}\right)^{-1} g_{2} k\right)\right\|, \quad \text { since } r\left(g_{1}\right)=r\left(g_{2}\right), \\
& =\left\|L_{k}^{*} v\left(\left(h^{r\left(g_{2}\right)}\right)^{-1} g_{2}\right)\right\|
\end{aligned}
$$




$$
=\left\|v\left(\left(h^{r\left(g_{2}\right)}\right)^{-1} g_{2}\right)\right\| .
$$

Therefore $\left\|v\left(h^{r(g)} g\right)\right\|$ is well-defined for $g \in G_{x} / G_{x}^{x}$ with $r(g) \in r(\mathcal{U})$. Then,

$$
\begin{aligned}
\left\|\vartheta_{x}(f) v\right\|^{2} & =\sum_{\substack{g \in G_{x} / G_{x}^{x} \\
r(g) \in r(\mathcal{U})}}\left\|\left(\vartheta_{x}(f) v\right)(g)\right\|^{2} \\
& =\sum_{\substack{g \in G_{x} / G_{x}^{x} \\
r(g) \in r(\mathcal{U})}}\left|f\left(h^{r(g)}\right)\right|^{2}\left\|v\left(\left(h^{r(g)}\right)^{-1} g\right)\right\|^{2} \\
& \leq\|f\|_{\infty}^{2} \sum_{\substack{g \in G_{x} / G_{x}^{x} \\
r(g) \in r(\mathcal{U})}}\left\|v\left(\left(h^{r(g)}\right)^{-1} g\right)\right\|^{2} .
\end{aligned}
$$

Note that for $g_{1}, g_{2}$ with $r\left(g_{1}\right) \in \mathcal{U}, g_{1}, g_{2}$ are equivalent if, and only if $\left(h^{r\left(g_{1}\right)}\right)^{-1} g_{1}$ and $\left(h^{r\left(g_{2}\right)}\right)^{-1} g_{2}$ are equivalent. In fact, suppose $g_{1}$ and $g_{2}$ are equivalent. Then $g_{1}=g_{2} k$ for some $k \in G_{x}^{x}$. Recall that $r\left(g_{1}\right)=r\left(g_{2}\right)$. Then $h^{r\left(g_{1}\right)}=h^{r\left(g_{2}\right)}$ and $\left(h^{r\left(g_{1}\right)}\right)^{-1} g_{1}=\left(h^{r\left(g_{2}\right)}\right)^{-1} g_{2} k$. Therefore $\left(h^{r\left(g_{1}\right)}\right) g_{1}$ and $\left(h^{r\left(g_{2}\right)}\right) g_{2}$ are equivalent.

Conversely, assume $\left(h^{r\left(g_{1}\right)}\right)^{-1} g_{1}$ and $\left(h^{r\left(g_{2}\right)}\right)^{-1} g_{2}$ are equivalent. Then there is $k \in G_{x}^{x}$ such that $\left(h^{r\left(g_{1}\right)}\right)^{-1} g_{1}=\left(h^{r\left(g_{2}\right)}\right)^{-1} g_{2} k$. Note that

$$
s\left(h^{r\left(g_{1}\right)}\right)=r\left(\left(h^{r\left(g_{1}\right)}\right)^{-1}\right)=r\left(\left(h^{r\left(g_{1}\right)}\right)^{-1} g_{1}\right)=r\left(\left(h^{r\left(g_{2}\right)}\right)^{-1} g_{2}\right)=r\left(\left(h^{r\left(g_{2}\right)}\right)^{-1}\right)=s\left(h^{r\left(g_{2}\right)}\right) .
$$

Since $h^{r\left(g_{1}\right)}, h^{r\left(g_{2}\right)} \in \mathcal{U}$ and $\mathcal{U}$ is an open bisection, it follows that $h^{r\left(g_{1}\right)}=h^{r\left(g_{2}\right)}$. Then $g_{1}=g_{2} k$. Therefore $g_{1}$ and $g_{2}$ are equivalent.

Then the set of elements $\left(h^{r(g)}\right)^{-1} g$ for $g \in G_{x} / G_{x}^{x}$ with $r(g) \in r(\mathcal{U})$ is a subset of the set $\left\{g: g \in G_{x} / G_{x}^{x}\right\}$. Hence, by 5.22 ,

$$
\begin{aligned}
\left\|\vartheta_{x}(f) v\right\|^{2} & \leq\|f\|_{\infty}^{2} \sum_{\substack{g \in G_{x} / G_{x}^{x} \\
r(g) \in r(\mathcal{U})}}\left\|v\left(\left(h^{r(g)}\right)^{-1} g\right)\right\|^{2} \\
& \leq\|f\|_{\infty}^{2} \sum_{g \in G_{x} / G_{x}^{x}}\|v(g)\|^{2}
\end{aligned}
$$




$$
\begin{aligned}
& =\|f\|_{\infty}^{2}\|v\|^{2} \\
& <\infty .
\end{aligned}
$$

Therefore, $\left\|\vartheta_{x}(f)\right\|<\infty$ for every $f \in C_{c}(G)$.

Now we prove that $\vartheta_{x}$ is a representation of $C_{c}(G)$ on $K_{x}$.

- $\vartheta_{x}\left(f_{1} \cdot f_{2}\right)=\vartheta_{x}\left(f_{1}\right) \vartheta_{x}\left(f_{2}\right)$.

Given $f_{1}, f_{2} \in C_{c}(G), v \in K_{x}, g \in G_{x}$

$$
\begin{aligned}
\left(\vartheta_{x}\left(f_{1} \cdot f_{2}\right) v\right)(g) & =\sum_{h \in G^{r(g)}}\left(f_{1} \cdot f_{2}\right)(h) v\left(h^{-1} g\right) \\
& =\sum_{h \in G^{r(g)}}\left(\sum_{k \in G^{r(h)}} f_{1}(k) f_{2}\left(k^{-1} h\right)\right) v\left(h^{-1} g\right), \quad \text { by 3.4 on page 55. } \\
& =\sum_{h \in G^{r(g)}} \sum_{k \in G^{r(g)}} f_{1}(k) f_{2}\left(k^{-1} h\right) v\left(h^{-1} g\right) \\
& =\sum_{k \in G^{r(g)}} f_{1}(k) \sum_{h \in G^{r(g)}} f_{2}\left(k^{-1} h\right) v\left(h^{-1} g\right) \\
& =\sum_{k \in G^{r(g)}} f_{1}(k) \sum_{h \in G^{r(g)}} f_{2}\left(k^{-1} h\right) v\left(h^{-1} k k^{-1} g\right) \\
& =\sum_{k \in G^{r(g)}} f_{1}(k) \sum_{h \in G^{r(g)}} f_{2}\left(k^{-1} h\right) v\left(\left(k^{-1} h\right)^{-1} k^{-1} g\right) .
\end{aligned}
$$

Making the change of variables $\tilde{h}=k^{-1} h$, then $\tilde{h} \in G^{r\left(k^{-1}\right)}=G^{r\left(k^{-1} g\right)}$. Then,

$$
\begin{aligned}
\left(\vartheta_{x}\left(f_{1} \cdot f_{2}\right) v\right)(g) & =\sum_{k \in G^{r(g)}} f_{1}(k) \sum_{\left.\tilde{h} \in G^{r(k-1} g\right)} f_{2}(\tilde{h}) v\left(\tilde{h}^{-1} k^{-1} g\right) \\
& =\sum_{k \in G^{r(g)}} f_{1}(k)\left(\vartheta_{x}\left(f_{2}\right) v\right)\left(k^{-1} g\right) \\
& =\vartheta_{x}\left(f_{1}\right)\left(\vartheta_{x}\left(f_{2}\right) v\right)(g) .
\end{aligned}
$$

- $\vartheta_{x}\left(f^{*}\right)=\vartheta_{x}(f)^{*}$ 
Given $v, w \in K_{x}$,

$$
\begin{aligned}
\left\langle\vartheta_{x}(f) v, w\right\rangle & =\sum_{g \in G_{x} / G_{x}^{x}}\left\langle\left(\vartheta_{x}(f) v\right)(g), w(g)\right\rangle \\
& =\sum_{g \in G_{x} / G_{x}^{x}} \sum_{h \in G^{r}(g)} f(h)\left\langle v\left(h^{-1} g\right), w(g)\right\rangle .
\end{aligned}
$$

On the other hand,

$$
\begin{aligned}
\left\langle v, \vartheta_{x}\left(f^{*}\right) w\right\rangle & =\sum_{g \in G_{x} / G_{x}^{x}}\left\langle v(g),\left(\vartheta_{x}\left(f^{*}\right) w\right)(g)\right\rangle \\
& =\sum_{g \in G_{x} / G_{x}^{x}} \sum_{h \in G^{r}(g)} \overline{f^{*}(h)}\left\langle v(g), w\left(h^{-1} g\right)\right\rangle \\
& =\sum_{g \in G_{x} / G_{x}^{x}} \sum_{h \in G^{r}(g)} f\left(h^{-1}\right)\left\langle v(g), w\left(h^{-1} g\right)\right\rangle
\end{aligned}
$$

Note that for every $g \in G_{x} / G_{x}^{x}, h \in G^{r(g)}$, there exist unique $\widetilde{g} \in G_{x} / G_{x}^{x}, \widetilde{h} \in G^{r(\widetilde{g})}$ satisfying

$$
\left\{\begin{array}{l}
\widetilde{h}=h^{-1} \\
\widetilde{g}=h^{-1} g .
\end{array}\right.
$$

Then we can make the change of variables in (5.23), obtaining

$$
\begin{aligned}
\left\langle v, \vartheta_{x}\left(f^{*}\right)\right\rangle & =\sum_{\widetilde{g} \in G_{x} / G_{x}^{x}} \sum_{\widetilde{h} \in G^{r(\widetilde{g})}} f(\widetilde{h})\left\langle v\left(\widetilde{h}^{-1} \widetilde{g}\right), w(\widetilde{g})\right\rangle \\
& =\left\langle\vartheta_{x}(f) v, w\right\rangle .
\end{aligned}
$$

Thus $\vartheta_{x}\left(f^{*}\right)=\vartheta(f)^{*}$.

Let $\zeta_{x}$ be defined by $\zeta_{x}(g)=\pi_{x}\left(u_{g}{ }^{*}\right) \xi_{x}$ if $g \in G_{x}^{x}$, and $\zeta_{x}(g)=0$ if $g \in G_{x} \backslash G_{x}^{x}$. Note that 
$\zeta_{x}$ is in $K_{x}$. Given $h \in G_{x}^{x}, g \in G_{x}, \zeta_{x}(g h)=0$ if $g \notin G_{x}^{x}$. If $g \in G_{x}^{x}$, by definition of $K_{x}$,

$$
\zeta_{x}(g h)=\pi_{x}\left(u_{g h}{ }^{*}\right) \xi_{x}=\pi_{x}\left(u_{h}^{*} u_{g}^{*}\right) \xi_{x}=\pi_{x}\left(u_{h}^{*}\right) \pi\left(u_{g}^{*}\right) \xi_{x}=\pi_{x}\left(u_{h}^{*}\right) \zeta_{x}(g)
$$

Moreover, $\left\|\zeta_{x}\right\|=\left\|\zeta_{x}(x)\right\|=\left\|\xi_{x}\right\|=1$. Let $\psi_{x}$ be the state on $C^{*}(G)$ defined by $\psi_{x}(f)=$ $\left\langle\vartheta_{x}(f) \zeta_{x}, \zeta_{x}\right\rangle$. Then, for $f \in C_{c}(G)$,

$$
\begin{aligned}
\psi_{x}(f) & =\sum_{g \in G_{x} / G_{x}^{x}}\left\langle\vartheta_{x}(f) \zeta_{x}(g), \zeta_{x}(g)\right\rangle \\
& =\left\langle\left(\vartheta_{x}(f) \zeta_{x}\right)(x), \zeta_{x}(x)\right\rangle \\
& =\sum_{h \in G^{x}} f(h)\left\langle\zeta_{x}\left(h^{-1} x\right), \zeta_{x}(x)\right\rangle \\
& =\sum_{h \in G^{x}} f(h)\left\langle\zeta_{x}\left(h^{-1}\right), \zeta_{x}(x)\right\rangle \\
& =\sum_{g \in G_{x}^{x}} f(g)\left\langle\pi_{x}\left(u_{g^{-1}}{ }^{*}\right) \xi_{x}, \pi_{x}\left(u_{x}^{*}\right) \xi_{x}\right\rangle, \quad \text { by definition of } \zeta_{x}, \\
& =\sum_{g \in G_{x}^{x}} f(g)\left\langle\pi_{x}\left(u_{g}^{* *}\right) \xi_{x}, \pi_{x}\left(u_{x}^{*}\right) \xi_{x}\right\rangle \\
& =\sum_{g \in G_{x}^{x}} f(g)\left\langle\pi_{x}\left(u_{g}\right) \xi_{x}, \pi_{x}\left(u_{x}\right) \xi_{x}\right\rangle \\
& =\sum_{g \in G_{x}^{x}} f(g) \varphi_{x}\left(u_{g}\right) .
\end{aligned}
$$

Note that $C_{0}\left(G^{(0)}\right)$ is in the centralizer of $\psi_{x}$. From Lemma 3.3 .16 , we have that $C_{c}\left(G^{(0)}\right)$ is dense in $C_{0}\left(G^{(0)}\right)$. Using the continuity of $\psi_{x}$, it is sufficient to show that $C_{c}\left(G^{(0)}\right)$ is in the centralizer of $\varphi$. Given $f \in C_{c}(G)$ and $h \in C_{c}\left(G^{(0)}\right)$, we have

$$
\psi_{x}(f \cdot h)=\sum_{g \in G_{x}^{x}}(f \cdot h)(g) \varphi_{x}\left(u_{g}\right)=\sum_{g \in G_{x}^{x}} f(g) h(x) \varphi_{x}\left(u_{g}\right)=\sum_{g \in G_{x}^{x}}(h \cdot f)(g) \varphi_{x}\left(u_{g}\right)=\psi_{x}(h \cdot f) .
$$

By assumption, the map $x \mapsto \psi_{x}(f)$ is $\mu$-measurable for every $f \in C_{c}(G)$. By Lemma 5.1.8, we can define a state $\varphi$ on $C^{*}(G)$ by $\varphi(f)=\int_{G^{(0)}} \psi_{x}(f) d \mu(x)$. 
Finally we show that if $\left(\mu,\left\{\varphi_{x}\right\}_{x}\right)$ and $\left(\widetilde{\mu},\left\{\widetilde{\varphi}_{x}\right\}_{x}\right)$ define the same state $\varphi$, then $\mu=\widetilde{\mu}$ and $\varphi_{x}=\widetilde{\varphi}_{x}$ for $\mu$-a.e. $x$.

Recall from Proposition 3.2 .12 that $G^{(0)}$ is clopen in $G$. Let $f \in C_{c}\left(G^{(0)}\right)$. Then,

$$
\begin{aligned}
\int_{G^{(0)}} \sum_{g \in G_{x}^{x}} f(g) \varphi_{x}\left(u_{g}\right) d \mu(x) & =\int_{G^{(0)}} \sum_{g \in G_{x}^{x}} f(g) \widetilde{\varphi}_{x}\left(u_{g}\right) d \widetilde{\mu}(x) \\
\int_{G^{(0)}} f(x) \varphi_{x}\left(u_{x}\right) d \mu(x) & =\int_{G^{(0)}} f(x) \widetilde{\varphi}_{x}\left(u_{x}\right) d \widetilde{\mu}(x), \quad \text { since } f \in C_{c}\left(G^{(0)}\right), \\
\int_{G^{(0)}} f(x) d \mu(x) & =\int_{G^{(0)}} f(x) d \widetilde{\mu}(x), \quad u_{x} \text { is the unity in } C^{*}\left(G_{x}^{x}\right) .
\end{aligned}
$$

Since $f$ is arbitrary, we have $\mu=\widetilde{\mu}$.

We will prove that $\varphi_{x}=\widetilde{\varphi}_{x}$ for $\mu$-a.e. $x$. Let $W \subset G$ be the set of $g \in G^{\prime}$ such that $x=r(g)$ and $\varphi_{x}\left(u_{g}\right) \neq \widetilde{\varphi}_{x}\left(u_{g}\right)$. Let $V \subset G^{(0)}$ be the set of $x \in G^{(0)}$ such that $\varphi_{x} \neq \widetilde{\varphi}_{x}$. Note that $V=r(W)$.

Given $g \in W$, let $\mathcal{U}_{g}$ be an open bisection containing $g$. Using the topological properties of $G$, we can assume the family $\left\{\mathcal{U}_{g}\right\}_{g \in W}$ is countable without loss of generality.

Given $f \in C_{c}\left(r\left(\mathcal{U}_{g}\right)\right)$, there exists $F \in C_{c}\left(\mathcal{U}_{g}\right)$ such that $f=\left.F \circ r\right|_{\mathcal{U}_{g}} ^{-1}$. We denote by $h^{x}=\left.r\right|_{\mathcal{U}_{g}} ^{-1}(x)$, hence $F\left(h^{x}\right)=f(x)$ for every $x \in r\left(\mathcal{U}_{g}\right)$. Hence,

$$
\begin{aligned}
& \int_{r\left(\mathcal{U}_{g} \cap G^{\prime}\right)} f(x)\left[\varphi_{x}\left(h^{x}\right)-\widetilde{\varphi}_{x}\left(h^{x}\right)\right] d \mu(x) \\
= & \int_{r\left(\mathcal{U}_{g} \cap G^{\prime}\right)} F\left(h^{x}\right)\left[\varphi_{x}\left(h^{x}\right)-\widetilde{\varphi}_{x}\left(h^{x}\right)\right] d \mu(x) \\
= & \int_{r\left(\mathcal{U}_{g} \cap G^{\prime}\right)} \sum_{g \in G_{x}^{x}} F(g)\left[\varphi_{x}\left(h^{x}\right)-\widetilde{\varphi}_{x}\left(h^{x}\right)\right] d \mu(x), \quad \text { since } h^{x} \in G^{\prime}, \\
= & \int_{r\left(\mathcal{U}_{g}\right)} \sum_{g \in G_{x}^{x}} F(g)\left[\varphi_{x}\left(h^{x}\right)-\widetilde{\varphi}_{x}\left(h^{x}\right)\right] d \mu(x) \\
= & \varphi(F)-\varphi(F) \\
= & 0 .
\end{aligned}
$$


Since $f$ is arbitrary, it follows that $\varphi_{x}\left(u_{h^{x}}\right)=\widetilde{\varphi}_{x}\left(u_{h^{x}}\right)$ for $\mu$-a.e. $x \in r\left(\mathcal{U}_{g} \cap G^{\prime}\right)$. Then $\mu\left(r\left(W \cap \mathcal{U}_{g}\right)\right)=0$. The family of $\mathcal{U}_{g}$ indexed by $g \in W$ is countable, and $V=\cup_{g \in W} r\left(W \cap \mathcal{U}_{g}\right)$. Then $\mu(V)=0$.

Remark 5.2.10. If we define a dynamics $\tau$ on $C^{*}(G)$ such that every function in $C_{0}\left(G^{(0)}\right)$ is $\tau$-invariant, it follows from Lemma 5.1.32, that every KMS state $\varphi$ on $C^{*}(G)$ has centralizer containing $C_{0}\left(G^{(0)}\right)$. In this case, we can apply the first Neshveyev's theorem.

\subsection{Second Theorem}

The second theorem shows the conditions that the pair $\left(\mu,\left\{\varphi_{x}\right\}_{x}\right)$ satisfies if its corresponding state is KMS. We begin by defining a dynamics $\tau$ on $C^{*}(G)$.

Definition 5.3.1. Let $G$ be a topological groupoid. A continuous $\mathbb{R}$-valued 1-cocyle on $G$ is a continuous function $c: G \rightarrow \mathbb{R}$ such that $c(g h)=c(g)+c(h)$ for every $(g, h) \in G^{(2)}$.

Now we will prove a lemma which will help us to show that the dynamics defined by the cocycle is well-defined.

Lemma 5.3.2. Let $G$ be a locally compact Hausdorff second countable étale groupoid. Let $\mathbb{K}$ be $\mathbb{R}$ or $\mathbb{C}$. Let $F: \mathbb{K} \times G \rightarrow \mathbb{C}$ be a continuous function. Let $\mathcal{U} \subset G$ be an open bisection and suppose there exists a compact set $K \subset \mathcal{U}$ such that $F(z, g)=0$ for every $z \in \mathbb{K}, g \notin K$.

Define for every $z \in \mathbb{K}$ the function $F_{z}: G \rightarrow \mathbb{C}$ by $F_{z}(g)=F(z, g)$. Then the map from $\mathbb{K}$ to $C_{c}(G)$ defined by $z \mapsto F_{z}$ is continuous with respect to the norm of $C^{*}(G)$.

Proof. We can assume $\mathbb{K}=\mathbb{C}$ without loss of generality. Note that each $F_{z}$ is continuous and its support is included in the compact set $K$.

Fix $z_{0} \in \mathbb{C}$. Note that $F$ is continuous at $\left(z_{0}, g\right)$ for every $g \in K$. Thus, for each $g \in K$ there exists $\delta_{g}>0$ and an open neighborhood $U_{g}$ of $g$ such that

$$
\left|F(z, h)-F\left(z_{0}, g\right)\right| \leq \frac{\varepsilon}{2}
$$


for every $(z, h)$ satisfying $z \in B_{\delta_{g}}\left(z_{0}\right)=\left\{w \in \mathbb{C}:\left|w-z_{0}\right|<\delta_{g}\right\}, h \in U_{g}$.

The family $\left\{B_{\delta_{g}}\left(z_{0}\right) \times U_{g}\right\}_{g \in K}$ forms an open cover for the compact set $\left\{z_{0}\right\} \times K$. Then there are $g_{1}, \ldots, g_{n} \in K$ such that $\left\{B_{\delta_{g_{i}}}\left(z_{0}\right) \times U_{g_{i}}\right\}_{i=1}^{n}$ covers $\left\{z_{0}\right\} \times K$. Let

$$
\delta=\frac{1}{2} \min \left\{\delta_{g_{1}}, \ldots, \delta_{g_{n}}\right\} .
$$

Now let $z \in \mathbb{C}$ such that $\left|z-z_{0}\right|<\delta$. Let $g \in K$. There exists $i \in\{1, \ldots, n\}$ such that

$$
\left(z_{0}, g\right) \in B_{\delta_{g_{i}}}\left(z_{0}\right) \times U_{g_{i}} .
$$

Since $\delta<\delta_{g_{i}}$, we have $(z, g) \in B_{\delta_{g_{i}}}\left(z_{0}\right) \times U_{g_{i}}$. Thus,

$$
\begin{aligned}
\left|F(z, g)-F\left(z_{0}, g\right)\right| & \leq\left|F(z, g)-F\left(z_{0}, g_{i}\right)\right|+\left|F\left(z_{0}, g_{i}\right)-F\left(z_{0}, g\right)\right| \\
& \leq \frac{\varepsilon}{2}+\frac{\varepsilon}{2} \\
& \leq \varepsilon
\end{aligned}
$$

because $(z, g),\left(z_{0}, g\right) \in B_{\delta_{g_{i}}} \times U_{g_{i}}$. Since $g \in K$ is arbitrary, we have

$$
\begin{aligned}
\left\|F_{z}-F_{z_{0}}\right\| & \leq\left\|F_{z}-F_{z_{0}}\right\|_{\infty}, \quad \text { from Proposition 3.3.11, } \\
& =\sup _{g \in K}\left|F_{z}(g)-F_{z_{0}}(g)\right| \\
& =\sup _{g \in K}\left|F(z, g)-F\left(z_{0}, g\right)\right| \\
& \leq \varepsilon .
\end{aligned}
$$

Therefore the map $z \mapsto F_{z}$ is continuous.

The next lemma is useful to prove that the elements of $C_{c}(G)$ are entire analytic for our dynamics.

Corollary 5.3.3. Using the same conditions of Lemma 5.3 .2 for $\mathbb{K}=\mathbb{C}$, suppose that for 
every $g \in \mathbb{C}$ the function $z \mapsto F(z, g)$ is differentiable. Define $F^{\prime}: \mathbb{C} \times G \rightarrow \mathbb{C}$ by

$$
F^{\prime}(z, g)=\frac{\partial F}{\partial z}(z, g)=\lim _{h \rightarrow 0} \frac{F(z+h, g)-F(z, g)}{h} .
$$

Suppose $F^{\prime}$ is continuous. For every $z \in \mathbb{C}$, define $F_{z}^{\prime}: G \rightarrow \mathbb{C}$ by $F_{z}(g)=F^{\prime}(z, g)$. Then we have the limit

$$
\lim _{h \rightarrow 0}\left\|\frac{F_{z+h}-F_{z}}{h}-F_{z}^{\prime}\right\|=0
$$

for every $z \in \mathbb{C}$.

Proof. Fix $z \in \mathbb{C}$. By definition, $F_{z}^{\prime}$ is supported on $K$. Define the function $H: \mathbb{C} \times G \rightarrow \mathbb{C}$ by

$$
H(h, g)= \begin{cases}\frac{F_{z+h}(g)-F_{z}(g)}{h}-F_{z}^{\prime}(g) & \text { if } h \neq 0 \\ 0 & \text { if } h=0\end{cases}
$$

This function continuous. Moreover, using the notation from of 5.3.2, we have that $H_{h}$ is supported on $K$ for every $h$. Then by Lemma 5.3.2,

$$
\begin{aligned}
0 & =\lim _{h \rightarrow 0}\left\|H_{h}-H_{0}\right\| \\
& =\lim _{h \rightarrow 0}\left\|H_{h}\right\| \\
& =\lim _{h \rightarrow 0}\left\|\frac{F_{z+h}-F_{z}}{h}-F_{z}^{\prime}\right\| .
\end{aligned}
$$

Now we define a dynamics on $C^{*}(G)$ for a continuous $\mathbb{R}$-valued cocycle. Throughout this section, the dynamics is fixed.

Lemma 5.3.4. Let $G$ be a locally compact Hausdorff second countable étale groupoid and $c: G \rightarrow \mathbb{R}$ a continuous $\mathbb{R}$-valued cocycle. Define $\tau=\left\{\tau_{t}\right\}_{t \in \mathbb{R}}$ by $\tau_{t}(f)(g)=e^{i t c(g)} f(g)$ for 
every $g \in G, f \in C_{c}(G)$. Then $\left(C^{*}(G), \tau\right)$ is a $\mathrm{C}^{*}$-dynamical system.

Proof. Fix $t \in \mathbb{R}$. Note that $\tau_{t}(f) \in C_{c}(G)$ for every $f \in C_{c}(G)$. By definition, $\tau_{t}$ is a linear map on $C_{c}(G)$.

- $\tau_{t}$ is a $*$-homomorphism.

Given $f_{1}, f_{2} \in C_{c}(G), g \in G$,

$$
\begin{aligned}
\tau_{t}\left(f_{1} \cdot f_{2}\right)(g) & =e^{i t c(g)}\left(f_{1} \cdot f_{2}\right)(g) \\
& =e^{i t c(g)} \sum_{g_{1} g_{2}=g} f_{1}\left(g_{1}\right) f_{2}\left(g_{2}\right) \\
& =\sum_{g_{1} g_{2}=g} e^{i t c\left(g_{1} g_{2}\right)} f_{1}\left(g_{1}\right) f_{2}\left(g_{2}\right) \\
& =\sum_{g_{1} g_{2}=g}\left(e^{i t c\left(g_{1}\right)} f_{1}\left(g_{1}\right)\right)\left(e^{i t c\left(g_{2}\right)} f_{2}\left(g_{2}\right)\right) \\
& =\sum_{g_{1} g_{2}=g} \tau_{t}\left(f_{1}\right)\left(g_{1}\right) \tau_{t}\left(f_{2}\right)\left(g_{2}\right) \\
& =\left(\tau_{t}\left(f_{1}\right) \cdot \tau_{t}\left(f_{2}\right)\right)(g) .
\end{aligned}
$$

Given $f \in C_{c}(G), g \in G$, we have

$$
\tau_{t}\left(f^{*}\right)(g)=e^{i t c(g)} f^{*}(g)=\overline{e^{-i t c(g)} f\left(g^{-1}\right)}=\overline{e^{i t c\left(g^{-1}\right)} f\left(g^{-1}\right)}=\overline{\tau_{t}(f)\left(g^{-1}\right)}=\tau_{t}(f)^{*}(g) .
$$

Thus $\tau_{t}$ is a $*$-homomorphism.

- $\tau_{t} \circ \tau_{s}=\tau_{t+s}$ and $\tau_{0}$ is the identity

Given $f \in C_{c}(G), g \in G, t, s \in \mathbb{R}$,

$$
\begin{aligned}
\tau_{t} \circ \tau_{s}(f)(g) & =\tau_{t}\left(\tau_{s}(f)\right)(g) \\
& =e^{i t c(g)} \tau_{s}(f)(g) \\
& =e^{i t c(g)} e^{i s c(g)} f(g)
\end{aligned}
$$




$$
\begin{aligned}
& =e^{i(t+s) c(g)} f(g) \\
& =\tau_{t+s}(f)(g) .
\end{aligned}
$$

By definition, $\tau_{0}$ is the identity.

Each $\tau_{t}$ is invertible. Therefore every $\tau_{t}$ is a $*$-automorphism.

- $\tau$ is strongly continuous

Let $f \in C_{c}(G)$. Let $K$ be the support of $f$. Assume there exists and open bisection $\mathcal{U}$ such that $K \subset \mathcal{U}$.

Define $F: \mathbb{R} \times G \rightarrow \mathbb{C}$ by $F(t, g)=\tau_{t}(f)(g)=e^{i t c(g)} f(g)$. Both $c$ and $f$ are continuous functions, then $F$ is continuous. Let $K$ be the compact support of $f$. Then $F(t, g)=0$ for every $t \in \mathbb{R}, g \notin K$. Using the notation of Lemma 5.3.2, we have $\tau_{t}(f)=F_{t}$. Therefore, by Lemma 5.3.2, the function $t \mapsto \tau_{t}(f)$ is continuous.

Now let $f \in C_{c}(G)$ be arbitrary. There are open bisections $\mathcal{U}_{1}, \ldots, \mathcal{U}_{n}$ and functions $f_{1}, \ldots, f_{n}$ such that each $f_{k} \in C_{c}\left(\mathcal{U}_{k}\right)$ and $f=f_{1}+\ldots+f_{n}$. Since the function $t \mapsto \tau_{t}\left(f_{k}\right)$ is continuous for every $k$ and each $\tau_{t}$ is linear, it follows that the function $t \mapsto \tau_{t}(f)$ is continuous.

Note that, for every $f \in C_{c}(G),\|\tau(f)\| \leq\|f\|$. In fact, let $\pi$ be a $*$-representation of $C_{c}(G)$. Then $\pi \circ \tau$ is an $*$-representation of $C_{c}(G)$. Then. by defintion of the norm on $C_{c}(G)$, we have

$$
\left\|\pi\left(\tau_{t}(f)\right)\right\|=\left\|\pi \circ \tau_{t}(f)\right\| f \| .
$$

Since $\pi$ is arbitrary, using the defintion of $\left\|\pi\left(\tau_{t}(f)\right)\right\|$, we have

$$
\left\|\tau_{t}(f)\right\| \leq\|f\| .
$$

Therefore, from Lemma 5.1.12, $\tau$ defines a dynamics on $C^{*}(G)$. 
Lemma 5.3.5. Let $G$ be a locally compact second countable étale groupoid. Fix $\beta>0$. Assume $\varphi$ is a $\mathrm{KMS}_{\beta}$-state. Then the centralizer of $\varphi$ contains $C_{0}\left(G^{(0)}\right)$.

Proof. Let $h \in C_{0}\left(G^{(0)}\right)$. Note that for every $x \in G^{(0)}, c(x)=c(x x)=2 c(x)=0$. Then $c(x)=0$. Since $h$ is supported on $G^{(0)}$, we have for every $t \in \mathbb{R}$,

$$
\tau_{t}(h)(g)=e^{i t c(g)} h(g)=h(g) \quad \text { for } g \in G .
$$

Then $\tau_{t}(h)=h$. Therefore, by Lemma 5.1.32, $h$ is in the centralizer of $\varphi$.

Lemma 5.3.5 shows that we can apply Theorem 5.2 .9 for every KMS state $\varphi$ on $C^{*}(G)$. Then $\varphi$ corresponds to a pair $\left(\mu,\left\{\varphi_{y}\right\}_{\left.y \in G^{(0)}\right)}\right.$ as shown in that theorem.

Lemma 5.3.6. Let $G$ be a locally compact Hausdorff second countable étale groupoid. Then every $f \in C_{c}(G)$ is entire analytic for $\tau$.

Proof. Let $f \in C_{c}(G)$. Since the set of entire analytic elements in $C^{*}(G)$ forms a vector space and each function in $C_{c}(G)$ can be decomposed as a sum of functions in $C_{c}(G)$ supported on open bisections, we can assume $f$ is supported on an open bisection without loss of generality. Let $K=\operatorname{supp}(f)$.

Define $F: \mathbb{C} \times G \rightarrow \mathbb{C}$ by $F(z, g)=e^{i z c(g)} f(g)$. Then $F$ is continuous. Define for every $z \in \mathbb{C}, F_{z}: G \rightarrow \mathbb{C}$ by

$$
F_{z}(g)=F(z, g)=e^{i z c(g)} f(g)
$$

Note that $F_{t}(f)=\tau_{t}(f)$ for every $t \in \mathbb{R}$. For every $z \in \mathbb{C}, F_{z}$ is continuous and supported on $K$. Analogously, define $F^{\prime}: \mathbb{C} \times G \rightarrow$ by

$$
F^{\prime}(z, g)=\frac{\partial F}{\partial z}(z, g)=i c(g) e^{i z c(g)} f(g)
$$


Then $F^{\prime}$ is continuous. From Corollary 5.3 .3 we have the limit

$$
\lim _{h \rightarrow 0}\left\|\frac{F_{z+h}-F_{z}}{h}-F_{z}^{\prime}\right\|=0 .
$$

Therefore $f$ is entire analytic. In this case, we can write $\tau_{z}(f)=F_{z}$ for $z \in \mathbb{C}$.

The following lemma proves some properties of compactly supported functions. These properties will be used in the proof of Neshneyev's theorem.

Lemma 5.3.7. Let $G$ be a locally compact Hausdorff second countable étale groupoid. Let $\mathcal{U}$ be a an open bisection and let $f_{1} \in C_{c}(\mathcal{U}), f_{2} \in C_{c}(G)$. Then, given $g \in G$,

$$
\begin{aligned}
& \left(f_{1} \cdot f_{2}\right)(g)= \begin{cases}f_{1}\left(h^{x}\right) f_{2}\left(\left(h^{x}\right)^{-1} g\right), & \text { for } x \in r(\mathcal{U}), g \in G^{x}, \\
0, & \text { if } r(g) \notin r(\mathcal{U}) .\end{cases} \\
& \left(f_{2} \cdot f_{1}\right)(g)= \begin{cases}f_{1}\left(h_{x}\right) f_{2}\left(g\left(h_{x}\right)^{-1}\right), & \text { for } x \in s(\mathcal{U}), g \in G_{x}, \\
0, & \text { if } s(g) \notin s(\mathcal{U}) .\end{cases}
\end{aligned}
$$

For $x \in r(\mathcal{U}), h^{x}$ denotes the unique element in $\mathcal{U} \cap G^{x}$. Analogously, for $x \in s(\mathcal{U}), h_{x}$ is the unique element in $\mathcal{U} \cap G_{x}$.

Proof. Let $f_{1} \in C_{c}(\mathcal{U}), f_{2} \in C_{c}(G)$.

Equation (5.25): Let $x \in G^{(0)}, g \in G^{x}$. Note that $G^{x} \cap \mathcal{U}=\varnothing$ if $x \notin r(\mathcal{U})$. From Lemma 3.3 .3 , we have

$$
\begin{aligned}
& \left(f_{1} \cdot f_{2}\right)(g)=\sum_{h \in G^{x}} f_{1}(h) f_{2}\left(h^{-1} g\right) \\
& =\sum_{h \in G^{x} \cap \mathcal{U}} f_{1}(h) f_{2}\left(h^{-1} g\right), \quad \text { since } f_{1} \in C_{c}(\mathcal{U}), \\
& = \begin{cases}f_{1}\left(h^{x}\right) f_{2}\left(\left(h^{x}\right)^{-1} g\right), & \text { for } x \in r(\mathcal{U}), g \in G^{x}, \\
0, & \text { if } r(g) \notin r(\mathcal{U}) .\end{cases}
\end{aligned}
$$


Equation (5.26): Let $x \in G^{(0)}, g \in G_{x}$. Note that $G_{x} \cap \mathcal{U}=\varnothing$ if $x \notin s(\mathcal{U})$. From Lemma 3.3 .3 , we have

$$
\begin{aligned}
& \left(f_{2} \cdot f_{1}\right)(g)=\sum_{h \in G_{x}} f_{2}\left(g h^{-1}\right) f_{1}(h) \\
& =\sum_{h \in G_{x} \cap \mathcal{U}} f_{2}\left(g h^{-1}\right) f_{1}(h), \quad \text { since } f_{1} \in C_{c}(\mathcal{U}) \text {, } \\
& = \begin{cases}f_{2}\left(g\left(h_{x}\right)^{-1}\right) f_{1}\left(h_{x}\right), & \text { if } x \in s(\mathcal{U}), \\
0, & \text { otherwise. }\end{cases}
\end{aligned}
$$

Lemma 5.3.8. Let $G$ be a locally compact Hausdorff second countable étale groupoid. Let $c$ be an $\mathbb{R}$-valued 1-cocyle. A measure $\mu$ on $G^{(0)}$ is quasi-invariant with Radon-Nikodym derivative $e^{c}$ if, and only if, for every open bisection $\mathcal{U} \subset G$, we have

$$
\frac{d T_{*} \mu}{d \mu}(x)=e^{c\left(h_{x}\right)}
$$

for $x \in s(\mathcal{U})$, where $h_{x} \in \mathcal{U}$ is the unique element such that $s\left(h_{x}\right)=x$ and $T: r(\mathcal{U}) \rightarrow s(\mathcal{U})$ is the homeomorphism defined by $T\left(r\left(h_{x}\right)\right)=x$. In particular, $T=\left.\left.s\right|_{\mathcal{U}} \circ r\right|_{\mathcal{U}} ^{-1}$.

Proof. Let $\mathcal{U}$ be an open bisection in $G$. Then $h_{x}=\left.s\right|_{\mathcal{U}} ^{-1}(x)$ for every $x \in s(\mathcal{U})$. Let $T: r(\mathcal{U}) \rightarrow s(\mathcal{U})$ such that $T\left(r\left(h_{x}\right)\right)=x$ for every $x \in s(\mathcal{U})$. Then, for every $x$,

$$
x=T\left(r\left(h_{x}\right)\right)=T\left(\left.r\right|_{\mathcal{U}}\left(h_{x}\right)\right)=T\left(\left.r\right|_{\mathcal{U}}\left(\left.s\right|_{\mathcal{U}} ^{-1}(x)\right)\right)
$$

Therefore $T=\left(\left.\left.r\right|_{\mathcal{U}} \circ s\right|_{\mathcal{U}} ^{-1}\right)^{-1}=\left.\left.s\right|_{\mathcal{U}} \circ r\right|_{\mathcal{U}} ^{-1}$.

First we show a formula which holds if, and only if, condition (5.27) is satisfied. Note that equation 5.27) holds if, and only if, for every $f \in C_{c}(s(\mathcal{U}))$,

$$
\int_{s(\mathcal{U})} f(x) d\left(T_{*} \mu\right)(x)=\int_{s(\mathcal{U})} e^{c\left(h_{x}\right)} f(x) d \mu(x) .
$$


Recall that a measure $\mu$ on $G^{(0)}$ is quasi-invariant with Radon-Nikodym derivative $e^{c}$ if, and only if, for every $\tilde{f} \in C_{c}(G)$,

$$
\int_{G} \tilde{f}(g) d \mu_{r}(g)=\int_{G} e^{c(g)} \tilde{f}(g) d \mu_{s}(g) .
$$

From the definition of $\mu_{r}, \mu_{s}$, this is equivalent to

$$
\int_{r(\mathcal{U})} \sum_{g \in G^{x}} \tilde{f}(g) d \mu(x)=\int_{s(\mathcal{U})} \sum_{g \in G_{x}} \tilde{f}(g) d \mu(x) .
$$

Since $\tilde{f} \in C_{c}(\mathcal{U})$, we can consider only $g \in \mathcal{U}$ in the integrals. Recall that $\mathcal{U} \cap G^{x}=\left\{h^{x}\right\}$, if $x \in r(\mathcal{U})$, where $h^{x}=\left.r\right|_{\mathcal{U}} ^{-1}(x)$. Analogously, $\mathcal{U} \cap G_{x}=\left\{h_{x}\right\}$ if $x \in s(\mathcal{U})$. Then we can rewrite $(5.29)$ as

$$
\int_{r(\mathcal{U})} \tilde{f}\left(h^{x}\right) d \mu(x)=\int_{s(\mathcal{U})} e^{c\left(h_{x}\right)} \tilde{f}\left(h_{x}\right) d \mu(x) .
$$

Therefore $\mu$ is quasi-invariant with Radon-Nikodym derivative $e^{c}$ if, and only if, 5.30 holds for every open bisection $\mathcal{U}, \tilde{f} \in C_{c}(\mathcal{U})$.

Note that for every $x \in r(\mathcal{U}), h^{x}=\left.r\right|_{\mathcal{U}} ^{-1}(x)=\left.\left.\left.s\right|_{\mathcal{U}} ^{-1} \circ s\right|_{\mathcal{U}} \circ r\right|_{\mathcal{U}} ^{-1}(x)=\left.s\right|_{\mathcal{U}} ^{-1}(T x)=h_{T x}$. Then 5.30 is equivalent to

$$
\int_{r(\mathcal{U})} \tilde{f}\left(h_{T x}\right) d \mu(x)=\int_{s(\mathcal{U})} e^{c\left(h_{x}\right)} \tilde{f}\left(h_{x}\right) d \mu(x) .
$$

There is a bijection from $C_{c}(s(\mathcal{U}))$ to $C_{c}(\mathcal{U})$ given by $f \mapsto \tilde{f}=\left.f \circ s\right|_{\mathcal{U}}$. This holds because $\left.s\right|_{\mathcal{U}}: \mathcal{U} \rightarrow s(\mathcal{U})$ is a homeomorphism. Therefore, for every $x \in s(\mathcal{U}), f(x)=f\left(s\left(h_{x}\right)\right)=$ $\tilde{f}\left(h_{x}\right)$. In the rest of this proof, given $f \in C_{c}(s(\mathcal{U}))$, we denote by $\tilde{f}$ its corresponding function in $C_{c}(\mathcal{U})$. Analogously, given $\tilde{f} \in C_{c}(\mathcal{U}), f$ is the corresponding function in $C_{c}(s(\mathcal{U})$ ).

Assume $\mu$ is quasi-invariant with Radon-Nikodym derivative $e^{c}$. Let $\mathcal{U}$ be an open bisec- 
tion of $G$. Let $f \in C_{c}(s(\mathcal{U}))$. Then

$$
\begin{aligned}
\int_{s(\mathcal{U})} f(x) d\left(T_{*} \mu\right)(x) & =\int_{s(\mathcal{U})} \tilde{f}\left(h_{x}\right) d\left(T_{*} \mu\right)(x) \\
& =\int_{r(\mathcal{U})} \tilde{f}\left(h_{T x}\right) d \mu(x), \text { from the definition of } T_{*} \mu \\
& =\int_{s(\mathcal{U})} e^{c\left(h_{x}\right)} \tilde{f}\left(h_{x}\right) d \mu(x), \text { by } 5.31 \\
& =\int_{s(\mathcal{U})} e^{c\left(h_{x}\right)} f(x) d \mu(x)
\end{aligned}
$$

Hence, (5.28) holds, then (5.27) holds.

Conversely, suppose 5.28 holds. Given an open bisection $\mathcal{U}, \tilde{f} \in C_{c}(\mathcal{U})$, we have

$$
\begin{aligned}
\int_{r(\mathcal{U})} \tilde{f}\left(h_{T x}\right) d \mu(x) & =\int_{r(\mathcal{U})} f(T x) d \mu(x) \\
& =\int_{s(\mathcal{U})} f(x) d\left(T_{*} \mu\right)(x) \\
& =\int_{s(\mathcal{U})} e^{c\left(h_{x}\right)} f(x) d \mu(x) \\
& =\int_{s(\mathcal{U})} e^{c\left(h_{x}\right)} \tilde{f}\left(h_{x}\right) d \mu(x) .
\end{aligned}
$$

Then (5.31) holds for $\tilde{f}$. Therefore, $\mu$ is quasi-invariant with Radon-Nikodym derivative $e^{c}$.

Lemma 5.3.9. Let $\varphi$ be a state on $C^{*}(G)$ with centralizer containing $C_{0}\left(G^{(0)}\right)$. Assume $\varphi$ corresponds to the pair $\left(\mu,\left\{\varphi_{x}\right\}_{x}\right)$. Then $\varphi$ is $\tau$-invariant if, and only if,

$$
\varphi_{x}\left(u_{g}\right)=0 \quad \text { for every } g \in G_{x}^{x} \backslash c^{-1}(0), \mu \text {-a.e. } x \text {. }
$$

Proof. Assume $\varphi$ is $\tau$-invariant. It follows from the continuity of $c$ that $c^{-1}(0)$ is closed. Let $\widetilde{g} \in G$ such that $c(\widetilde{g}) \neq 0$. Let $t \in \mathbb{R}$ such that $t c(\widetilde{g}) \in(0,2 \pi)$. Then $1-e^{i t c(\widetilde{g})} \neq 0$. There exists an open bisection $\mathcal{U}$ containing $\widetilde{g}$ such that $1-e^{i t c(g)} \neq 0$ for every $g \in \mathcal{U}$. 
Let $f \in C_{c}(s(\mathcal{U}))$ and define $\tilde{f}=\left.f \circ s\right|_{\mathcal{U}} \in C_{c}(\mathcal{U})$. Let $x \in G^{(0)}$. Suppose $g \in G_{x}^{x} \cap \mathcal{U}$. Then $x \in s(\mathcal{U})$ and $g=h_{x}$, where $h_{x}=\left.s\right|_{\mathcal{U}} ^{-1}(x)$ denotes the unique element in $\mathcal{U} \cap G_{x}$. Hence, we can write

$$
\begin{aligned}
\varphi(\widetilde{f}) & =\int_{G^{(0)}} \sum_{g \in G_{x}^{x}} \widetilde{f}(g) \varphi_{x}\left(u_{g}\right) d \mu(x) \\
& =\int_{G^{(0)}} \sum_{g \in G_{x}^{x} \cap \mathcal{U}} \widetilde{f}(g) \varphi_{x}\left(u_{g}\right) d \mu(x) \\
& =\int_{s(\mathcal{U})} \sum_{g \in G_{x}^{x} \cap \mathcal{U}} \widetilde{f}(g) \varphi_{x}\left(u_{g}\right) d \mu(x) \\
& =\int_{s(\mathcal{U})} \sum_{g \in G_{x} \cap \mathcal{U}} \chi_{G^{\prime}}(g) \widetilde{f}(g) \varphi_{x}\left(u_{g}\right) d \mu(x) \\
& =\int_{s(\mathcal{U})} \chi_{G^{\prime}}\left(h_{x}\right) \widetilde{f}\left(h_{x}\right) \varphi_{x}\left(u_{h_{x}}\right) d \mu(x) \\
& =\int_{s(\mathcal{U})} \chi_{G^{\prime}}\left(h_{x}\right) \varphi_{x}\left(u_{h_{x}}\right) f(x) d \mu(x), \quad \text { by definition of } f .
\end{aligned}
$$

Analogously,

$$
\varphi\left(\tau_{t}(\widetilde{f})\right)=\int_{s(\mathcal{U})} e^{i t c\left(h_{x}\right)} \chi_{G^{\prime}}\left(h_{x}\right) \varphi_{x}\left(u_{h_{x}}\right) f(x) d \mu(x) .
$$

Since $\varphi\left(\tau_{t}(\widetilde{f})\right)=\varphi(\widetilde{f})$ by hypothesis, then from equations (5.33) and 5.34 we have

$$
\int_{s(\mathcal{U})}\left[1-e^{i t c\left(h_{x}\right)}\right] \chi_{G^{\prime}}\left(h_{x}\right) \varphi_{x}\left(u_{h_{x}}\right) f(x) d \mu(x)=0 .
$$

The function $f \in C_{c}(s(\mathcal{U}))$ is arbitrary and each $1-e^{i t c\left(h_{x}\right)} \neq 0$. Then, for $\mu$-a.e. $x \in s(\mathcal{U})$,

$$
\chi_{G^{\prime}}\left(h_{x}\right) \varphi_{x}\left(u_{h_{x}}\right)=0
$$

or equivalently, for $\mu$-a.e. $x \in s(\mathcal{U}), g \in \mathcal{U} \cap G_{x}$

$$
\chi_{G^{\prime}}(g) \varphi_{x}\left(u_{g}\right)=0 .
$$


Note that $G \backslash c^{-1}(0)$ can be covered by a countable family of open bisections $\left\{\mathcal{U}_{n}\right\}_{n \in \mathbb{N}}$ such that (5.35) holds for each $\mathcal{U}_{n}$. Therefore, for $\mu$-a.e. $x \in G^{(0)}$, for all $g \in G_{x} \backslash c^{-1}(0)$,

$$
\chi_{G^{\prime}}(g) \varphi_{x}\left(u_{g}\right)=0 .
$$

Thus, for $\mu$-a.e. $x \in G^{(0)}$, for all $g \in G_{x}^{x}, \varphi_{x}\left(u_{g}\right)=0$.

Conversely, assume 5.32 holds. Let $f \in C_{c}(G)$. Then, for $\mu$-a.e. $x \in G^{(0)}$ and all $g \in G_{x}^{x}$, $\varphi\left(u_{g}\right) \neq 0$ implies $c(g)=0$. Then, for $\mu$-a.e. $x \in G^{(0)}$,

$$
\sum_{g \in G_{x}^{x}} f(g) \varphi_{x}\left(u_{g}\right)=\sum_{g \in G_{x}^{x}} e^{i t c(g)} f(g) \varphi_{x}\left(u_{g}\right) .
$$

Therefore,

$$
\begin{aligned}
\varphi\left(\tau_{t}(f)\right) & =\int_{G^{(0)}} \sum_{g \in G_{x}^{x}} e^{i t c(g)} f(g) \varphi_{x}\left(u_{g}\right) d \mu(x) \\
& =\int_{G^{(0)}} \sum_{g \in G_{x}^{x}} f(g) \varphi_{x}\left(u_{g}\right) d \mu(x) \\
& =\varphi(f) .
\end{aligned}
$$

Since $\varphi$ and $\varphi \circ \tau_{t}$ are continuous functions and $C_{c}(G)$ is dense in $C^{*}(G)$, it follows that $\varphi\left(\tau_{t}(a)\right)=\varphi(a)$ for every $a \in C^{*}(G)$. In other words, $\varphi$ is $\tau$-invariant.

Now we prove Neshveyev's second theorem. Note that in this theorem we assume a different definition for KMS-states. Given $\beta \in \mathbb{R}$, a state $\varphi$ on a $\mathrm{C}^{*}$-algebra $A$ is a $\mathrm{KMS}_{\beta^{-}}$ state if $\varphi$ is $\tau$-invariant and $\varphi\left(a \tau_{i \beta}(b)\right)=\varphi(b a)$ for a dense subset of analytic elements $a, b \in A$. This definition corresponds to item (ii) in Proposition 5.1.29, so it is equivalent to the definition introduced in Section 5.1 when $\beta \neq 0$.

Theorem 5.3.10. (Neshveyev) Let $G$ be a locally compact second countable Hausdorff étale groupoid. Let $c$ be a continuous $\mathbb{R}$-valued 1-cocycle on $G$ and $\tau$ be the dynamics on $C^{*}(G)$ defined by $\tau_{t}(f)(g)=e^{i t c(g)} f(g)$ for $f \in C_{c}(G), g \in G$. Fix $\beta \in \mathbb{R}$. Then there exists a one- 


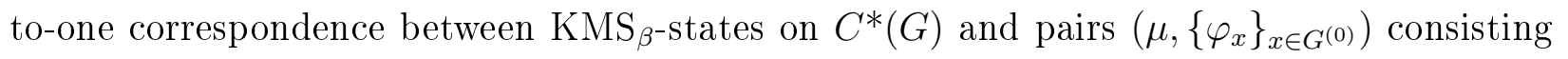
of a probability measure $\mu$ on $G^{(0)}$ and a $\mu$-measurable field of states $\varphi_{x}$ on $C^{*}\left(G_{x}^{x}\right)$ such that:

(i) $\mu$ is quasi-invariant with Radon-Nikodym derivative $e^{-\beta c}$;

(ii) $\varphi_{x}\left(u_{g}\right)=\varphi_{r(h)}\left(u_{h g h^{-1}}\right)$ for every $g \in G_{x}^{x}$ and $h \in G_{x}$, for $\mu$-a.e. $x$; in particular, $\varphi_{x}$ is tracial for $\mu$-a.e. $x$;

(iii) $\varphi_{x}\left(u_{g}\right)=0$ for all $g \in G_{x}^{x} \backslash c^{-1}(0)$, for $\mu$-a.e. $x$.

Proof. From Lemma 5.3.5, the centralizer of any $\tau$-KMS-state $\varphi$ contains $C_{0}\left(G^{(0)}\right)$. By Theorem 5.2.9, $\varphi$ is defined by a pair $\left(\mu,\left\{\varphi_{x}\right\}_{x}\right)$ consisting of a probability measure $\mu$ on $G^{(0)}$ and a $\mu$-measurable field of states $\varphi_{x}$ on $C^{*}\left(G_{x}^{x}\right)$. It follows from Lemma 5.3 .9 that property (iii) is satisfied if and only if $\varphi$ is $\tau$-invariant.

Therefore we have to prove that properties (i) and (ii) are satisfied if, and only if,

$$
\varphi\left(f_{1} \cdot f_{2}\right)=\varphi\left(f_{2} \cdot \tau_{i \beta}\left(f_{1}\right)\right), \quad \text { for every } f_{1}, f_{2} \in C_{c}(G)
$$

Each function in $C_{c}(G)$ can be decomposed as a finite sum of continuous functions supported on open bisections from Lemma 3.3.5. Every function in $C_{c}(G)$ is entire analytic by by Lemma 5.3.6. Note that $\tau_{i \beta}$ is linear on $C_{c}(G)$ by definition. Therefore (5.37) holds if, and only if, for every open bisection $\mathcal{U}$ we have

$$
\varphi\left(f_{1} \cdot f_{2}\right)=\varphi\left(f_{2} \cdot \tau_{i \beta}\left(f_{1}\right)\right), \quad \text { for every } f_{1} \in C_{c}(\mathcal{U}), f_{2} \in C_{c}(G)
$$

We will show (5.38) is equivalent to another equation and we will use this to prove the equivalence between (5.38) and conditions (i) and (ii). Given an open bisection $\mathcal{U}$, define $h^{x}=\left.r\right|_{\mathcal{U}} ^{-1}(x)$ for every $x \in r(\mathcal{U})$. Analogously, define $h_{x}=\left.s\right|_{\mathcal{U}} ^{-1}(x)$ for $x \in s(\mathcal{U})$. Then, for 
every $x \in r(\mathcal{U})$,

$$
\begin{aligned}
T x & =\left.\left.s\right|_{\mathcal{U}} \circ r\right|_{\mathcal{U}} ^{-1}(x)=s\left(h^{x}\right), \quad \text { and } \\
h_{T x} & =\left.s\right|_{\mathcal{U}} ^{-1}(T x)=\left.s\right|_{\mathcal{U}} ^{-1}\left(\left.\left.s\right|_{\mathcal{U}} \circ r\right|_{\mathcal{U}} ^{-1}(x)\right)=\left.r\right|_{\mathcal{U}} ^{-1}(x)=h^{x} .
\end{aligned}
$$

Suppose (5.37) holds. Let $f_{1} \in C_{c}(\mathcal{U}), f_{2} \in C_{c}(G)$. It follows that

$$
\begin{aligned}
\varphi\left(f_{1} \cdot f_{2}\right) & =\int_{G^{(0)}} \sum_{g \in G_{x}^{x}}\left(f_{1} \cdot f_{2}\right)(g) \varphi_{x}\left(u_{g}\right) d \mu(x) \\
& =\int_{r(\mathcal{U})} \sum_{g \in G_{x}^{x}} f_{1}\left(h^{x}\right) f_{2}\left(\left(h^{x}\right)^{-1} g\right) \varphi_{x}\left(u_{g}\right) d \mu(x), \quad \text { from Lemma 5.3.7, } \\
& =\int_{r(\mathcal{U})} f_{1}\left(h^{x}\right) \sum_{g \in G_{x}^{x}} f_{2}\left(\left(h^{x}\right)^{-1} g\right) \varphi_{x}\left(u_{g}\right) d \mu(x) .
\end{aligned}
$$

Since $\tau_{i \beta}\left(f_{1}\right) \in C_{c}(\mathcal{U})$, we can apply Lemma 5.3.7 and obtain

$$
\begin{aligned}
\varphi\left(f_{2} \cdot \tau_{i \beta}\left(f_{1}\right)\right) & =\int_{G^{(0)}} \sum_{g \in G_{x}^{x}}\left(f_{2} \cdot \tau_{i \beta}\left(f_{1}\right)\right)(g) \varphi_{x}\left(u_{g}\right) d \mu(x) \\
& =\int_{s(\mathcal{U})} \sum_{g \in G_{x}^{x}} \tau_{i \beta}\left(f_{1}\right)\left(h_{x}\right) f_{2}\left(g\left(h_{x}\right)^{-1}\right) \varphi_{x}\left(u_{g}\right) d \mu(x), \quad \text { from Lemma 5.3.7. } \\
& =\int_{s(\mathcal{U})} \sum_{g \in G_{x}^{x}} e^{-\beta c\left(h_{x}\right)} f_{1}\left(h_{x}\right) f_{2}\left(g\left(h_{x}\right)^{-1}\right) \varphi_{x}\left(u_{g}\right) d \mu(x) \\
& =\int_{s(\mathcal{U})} e^{-\beta c\left(h_{x}\right)} f_{1}\left(h_{x}\right) \sum_{g \in G_{x}^{x}} f_{2}\left(g\left(h_{x}\right)^{-1}\right) \varphi_{x}\left(u_{g}\right) d \mu(x) .
\end{aligned}
$$

Therefore (5.38) is equivalent to the following equation for $f_{1} \in C_{c}(\mathcal{U}), f_{2} \in C_{c}(G), \mathcal{U}$ open bisection.

$$
\int_{r(\mathcal{U})} f_{1}\left(h^{x}\right) \sum_{g \in G_{x}^{x}} f_{2}\left(\left(h^{x}\right)^{-1} g\right) \varphi_{x}\left(u_{g}\right) d \mu(x)=\int_{s(\mathcal{U})} e^{-\beta c\left(h_{x}\right)} f_{1}\left(h_{x}\right) \sum_{g \in G_{x}^{x}} f_{2}\left(g\left(h_{x}\right)^{-1}\right) \varphi_{x}\left(u_{g}\right) d \mu(x) .
$$


Suppose 5.39 holds. Let $f \in C_{c}(s(\mathcal{U}))$, then we can define $\tilde{f}_{1} \in C_{c}(\mathcal{U})$ such that $\left.f\right|_{\mathcal{U}}=\left.f \circ s\right|_{\mathcal{U}}$. Let $\tilde{f}_{2}=\widetilde{f}_{1}{ }^{*}$. Then $\tilde{f}_{2} \in C_{c}\left(\mathcal{U}^{-1}\right)$ by Lemma 3.3.8.

Let $x \in r(\mathcal{U}), g \in G_{x}^{x}$ such that $\widetilde{f}_{2}\left(\left(h^{x}\right)^{-1} g\right) \neq 0$. Then $\left(h^{x}\right)^{-1} g \in \mathcal{U}^{-1}$ which implies $g^{-1} h^{x} \in \mathcal{U}$. Recall that $\mathcal{U}$ is an open bisection and $h^{x} \in \mathcal{U}$. Moreover, $s\left(g^{-1} h^{x}\right)=s\left(h^{x}\right)$, then $g^{-1} h^{x}=h^{x}$, hence $g=x$. Therefore, for all $x \in r(\mathcal{U})$, we have

$$
\sum_{g \in G_{x}^{x}} \widetilde{f}_{2}\left(\left(h^{x}\right)^{-1} g\right) \varphi_{x}\left(u_{g}\right)=\widetilde{f}_{2}\left(\left(h^{x}\right)^{-1} x\right) \varphi_{x}\left(u_{x}\right)=\widetilde{f}_{2}\left(\left(h^{x}\right)^{-1}\right) .
$$

Therefore, for $\widetilde{f}_{1}, \widetilde{f}_{2}$, we can rewrite 5.39 as

$$
\begin{aligned}
\int_{s(\mathcal{U})} e^{-\beta c\left(h_{x}\right)} \tilde{f}_{1}\left(h_{x}\right) \widetilde{f}_{2}\left(\left(h_{x}\right)^{-1}\right) d \mu(x) & =\int_{r(\mathcal{U})} \widetilde{f}_{1}\left(h^{x}\right) \widetilde{f}_{2}\left(\left(h^{x}\right)^{-1}\right) d \mu(x) \\
& =\int_{r(\mathcal{U})} \widetilde{f}_{1}\left(h_{T x}\right) \widetilde{f}_{2}\left(\left(h_{T x}\right)^{-1}\right) d \mu(x),
\end{aligned}
$$

because $h_{T x}=h^{x}$ for $x \in r(\mathcal{U})$. Using the definition of $\widetilde{f}_{1}$ and $\widetilde{f}_{2}$, we have for every $x \in s(\mathcal{U})$,

$$
\begin{aligned}
\tilde{f}_{1}\left(h_{x}\right) & =\left.f \circ s\right|_{\mathcal{U}}\left(h_{x}\right)=\left.f \circ s\right|_{\mathcal{U}}\left(\left.s\right|_{\mathcal{U}} ^{-1}(x)\right)=f(x), \\
\widetilde{f}_{2}\left(\left(h_{x}\right)^{-1}\right) & =\widetilde{f}_{1}^{*}\left(\left(h_{x}\right)^{-1}\right)=\overline{\widetilde{f}_{1}\left(h_{x}\right)}=\overline{f(x)} .
\end{aligned}
$$

Then we can replace the values in the integrals in 5.40 and obtain

$$
\int_{s(\mathcal{U})} e^{-\beta c\left(h_{x}\right)}|f(x)|^{2} d \mu(x)=\int_{r(\mathcal{U})}|f(T x)|^{2} d \mu(x) .
$$

Since $f$ is arbitrary, it follows that for every $f \in C_{c}(s(\mathcal{U}))$,

$$
\int_{s(\mathcal{U})} e^{-\beta c\left(h_{x}\right)} f(x) d \mu(x)=\int_{r(\mathcal{U})} f(T x) d \mu(x) .
$$

Hence $\frac{d T_{*} \mu}{d \mu}(x)=e^{-\beta c\left(h_{x}\right)}$. It follows from Lemma 5.3 .8 that property (i) holds.

Now we show that property (ii) is satisfied. Let $\mathcal{U}$ be an open bisection and $f \in C_{c}(r(\mathcal{U})$, 
define $f_{1} \in C_{c}(\mathcal{U})$ such that $\left.f_{1}\right|_{\mathcal{U}}=\left.f \circ r\right|_{\mathcal{U}}$. Then $f_{1}\left(h^{x}\right)=f(x)$. Given $f_{2} \in C_{c}(G)$ arbitrary, define the function $F: G^{(0)} \rightarrow \mathbb{C}$ by

$$
F(x)=\sum_{g \in G_{x}^{x}}\left(f_{2} \cdot f_{1}\right)(g) \varphi_{x}\left(u_{g}\right) .
$$

This function is $\mu$-measurable because $f_{2} \cdot f_{1} \in C_{c}(G)$ and $\left\{\varphi_{x}\right\}_{x}$ is a $\mu$-measurable field of states. Moreover, by Lemma 5.3.7.

$$
F(x)= \begin{cases}f_{1}\left(h_{x}\right) \sum_{g \in G_{x}^{x}} f_{2}\left(g\left(h_{x}\right)^{-1}\right) \varphi_{x}\left(u_{g}\right), & \text { if } x \in s(\mathcal{U}) \\ 0, & \text { otherwise }\end{cases}
$$

Then

$$
\begin{aligned}
\int_{r(\mathcal{U})} f(x) \sum_{g \in G_{T x}^{T x}} f_{2}\left(g\left(h^{x}\right)^{-1}\right) \varphi_{T x}\left(u_{g}\right) d \mu(x) & =\int_{r(\mathcal{U})} f_{1}\left(h^{x}\right) \sum_{g \in G_{T x}^{T x}} f_{2}\left(g\left(h^{x}\right)^{-1}\right) \varphi_{T x}\left(u_{g}\right) d \mu(x) \\
& =\int_{r(\mathcal{U})} f_{1}\left(h_{T x}\right) \sum_{g \in G_{T x}^{T x}} f_{2}\left(g\left(h_{T x}\right)^{-1}\right) \varphi_{T x}\left(u_{g}\right) d \mu(x) \\
& =\int_{r(\mathcal{U})} F(T x) d \mu(x) \\
& =\int_{s(\mathcal{U})} F(x) d T_{*} \mu(x) \\
& =\int_{s(\mathcal{U})} e^{-\beta c\left(h_{x}\right)} F(x) d \mu(x),
\end{aligned}
$$

by property (i) that we already proved. Then,

$$
\begin{aligned}
& =\int_{s(\mathcal{U})} e^{-\beta c\left(h_{x}\right)} f_{1}\left(h_{x}\right) \sum_{g \in G_{x}^{x}} f_{2}\left(g\left(h_{x}\right)^{-1}\right) \varphi_{x}\left(u_{g}\right) d \mu(x), \\
& =\int_{r(\mathcal{U})} f_{1}\left(h^{x}\right) \sum_{g \in G_{x}^{x}} f_{2}\left(\left(h^{x}\right)^{-1} g\right) \varphi_{x}\left(u_{g}\right) d \mu(x),
\end{aligned}
$$


by (5.39). Then,

$$
=\int_{r(\mathcal{U})} f(x) \sum_{g \in G_{x}^{x}} f_{2}\left(\left(h^{x}\right)^{-1} g\right) \varphi_{x}\left(u_{g}\right) d \mu(x),
$$

if we make the change of variables $\widetilde{g}=h^{x} g\left(h^{x}\right)^{-1}$, we get

$$
=\int_{r(\mathcal{U})} f(x) \sum_{\widetilde{g} \in G_{T x}^{T x}} f_{2}\left(\widetilde{g}\left(h^{x}\right)^{-1}\right) \varphi_{x}\left(u_{\left(h^{x}\right)^{-1} \widetilde{g} h^{x}}\right) d \mu(x) .
$$

Assume $\mathcal{U}$ has compact closure. Let $\mathcal{V}$ be an open bisection with compact closure such that $s(\mathcal{V}) \subset s(\mathcal{U})$. We can assume $s(\mathcal{V})=s(\mathcal{U})$ without loss of generality. In fact, if this is not the case, just replace $\mathcal{U}$ by $\left.s\right|_{\mathcal{U}} ^{-1} \circ s(\mathcal{V}) . \mathcal{V U}^{-1}$ is an open bisection from Lemmas 3.3.6 and 3.3.7.

Assume $f_{2} \in C_{c}(G)$ is such that $f_{2}$ is positive on $\mathcal{V U}^{-1}$ and vanishes outside this set. Since $f \in C_{c}(r(\mathcal{U}))$ is arbitrary, we have for $\mu$-a.e. $x$,

$$
\sum_{g \in G_{T x}^{T x}} f_{2}\left(g\left(h^{x}\right)^{-1}\right)\left[\varphi_{x}\left(u_{\left(h^{x}\right)^{-1} g h^{x}}\right)-\varphi_{T x}\left(u_{g}\right)\right]=0 .
$$

Let $x \in r(\mathcal{U})$ be such that $(5.41)$ holds. Let $g \in \mathcal{V} \cap G_{T x}$. Then $g\left(h^{x}\right)^{-1}$ is the unique element in $\mathcal{V U}^{-1}$. Thus we can write 5.41 as

$$
\varphi_{x}\left(u_{\left(h^{x}\right)^{-1} g h^{x}}\right)=\varphi_{T x}\left(u_{g}\right) \text { for } g \in G_{T x}^{T x} \cap \mathcal{V}, \mu \text {-a.e. } x \in r(\mathcal{U}) \text {. }
$$

Since the preimage of $s(\mathcal{U})$ under $s$ can be covered by a countable family of open bisections $\mathcal{V}$ with compact closure such that $s(\mathcal{V}) \subset s(\mathcal{U})$, it follows that for $\mu$-a.e. $x \in r(\mathcal{U}), g \in G_{T x}^{T x}$, we have

$$
\varphi_{x}\left(u_{\left(h^{x}\right)^{-1} g h^{x}}\right)=\varphi_{T x}\left(u_{g}\right)=\varphi_{s\left(h^{x}\right)}\left(u_{g}\right) .
$$


Note that the set

$$
\begin{aligned}
& \left\{x \in G^{(0)}: \varphi_{x}\left(u_{g}\right) \neq \varphi_{r(h)}\left(u_{h g h^{-1}}\right) \text { for some } g \in G_{x}^{x}, h \in G_{x}\right\} \\
= & \left\{x \in G^{(0)}: \varphi_{x}\left(u_{g}\right) \neq \varphi_{r\left(h^{-1}\right)}\left(u_{h^{-1} g h}\right) \text { for some } g \in G_{x}^{x}, h \in G^{x}\right\} \\
= & \left\{x \in G^{(0)}: \varphi_{x}\left(u_{g}\right) \neq \varphi_{s(h)}\left(u_{h^{-1} g h}\right) \text { for some } g \in G_{x}^{x}, h \in G^{x}\right\} \\
= & \bigcup_{\mathcal{U}}\left\{x \in G^{(0)}: \varphi_{x}\left(u_{g}\right) \neq \varphi_{s(h)}\left(u_{h^{-1} g h}\right) \text { for some } g \in G_{x}^{x}, h \in G^{x} \cap \mathcal{U}\right\} \\
= & \bigcup_{\mathcal{U}}\left\{x \in r(\mathcal{U}): \varphi_{x}\left(u_{g}\right) \neq \varphi_{s(h)}\left(u_{h^{-1} g h}\right) \text { for some } g \in G_{x}^{x}, h \in G^{x} \cap \mathcal{U}\right\} \\
= & \bigcup_{\mathcal{U}}\left\{x \in r(\mathcal{U}): \varphi_{x}\left(u_{g}\right) \neq \varphi_{s\left(h^{x}\right)}\left(u_{\left(h^{x}\right)^{-1} g h^{x}}\right) \text { for some } g \in G_{x}^{x}\right\} \\
= & \bigcup_{\mathcal{U}}\left\{x \in r(\mathcal{U}): \varphi_{x}\left(u_{g}\right) \neq \varphi_{T x}\left(u_{\left(h^{x}\right)^{-1} g h^{x}}\right) \text { for some } g \in G_{x}^{x}\right\}
\end{aligned}
$$

has measure zero. Here $\mathcal{U}$ ranges over a countable open cover of $G$ such that $r, s$ are injective on $\mathcal{U}$. Therefore property (ii) holds.

Conversely, assume properties (i), and (ii) are satisfied. Given an open bisection $\mathcal{U}$, let $f_{1} \in C_{c}(\mathcal{U}), f_{2} \in C_{c}(G)$. Then

$$
\begin{aligned}
& \int_{r(\mathcal{U})} f_{1}\left(h^{x}\right) \sum_{g \in G_{x}^{x}} f_{2}\left(\left(h^{x}\right)^{-1} g\right) \varphi_{x}\left(u_{g}\right) d \mu(x) \\
& =\int_{r(\mathcal{U})} f_{1}\left(h^{x}\right) \sum_{\tilde{g} \in G_{T x}^{T x}} f_{2}\left(\tilde{g}\left(h^{x}\right)^{-1}\right) \varphi_{x}\left(u_{h^{x} \tilde{g}\left(h^{x}\right)^{-1}}\right) d \mu(x) \quad \text {, making } \tilde{g}=\left(h^{x}\right)^{-1} g h^{x}, \\
& =\int_{r(\mathcal{U})} f_{1}\left(h^{x}\right) \sum_{\tilde{g} \in G_{T x}^{T x}} f_{2}\left(\tilde{g}\left(h^{x}\right)^{-1}\right) \varphi_{r\left(h^{x}\right)}\left(u_{h^{x} \tilde{g}\left(h^{x}\right)^{-1}}\right) d \mu(x) \\
& =\int_{r(\mathcal{U})} f_{1}\left(h_{T x}\right) \sum_{\tilde{g} \in G_{T x}^{T x}} f_{2}\left(\tilde{g}\left(h_{T x}\right)^{-1}\right) \varphi_{r\left(h_{T x}\right)}\left(u_{h_{T x} \tilde{g}\left(h_{T x}\right)^{-1}}\right) d \mu(x) \\
& =\int_{r(\mathcal{U})} f_{1}\left(h_{T x}\right) \sum_{\tilde{g} \in G_{T x}^{T x}} f_{2}\left(\tilde{g}\left(h_{T x}\right)^{-1}\right) \varphi_{T x}\left(u_{\tilde{g}}\right) d \mu(x), \quad \text { from property (ii), } \\
& =\int_{s(\mathcal{U})} e^{-\beta c\left(h_{x}\right)} f_{1}\left(h_{x}\right) \sum_{\tilde{g} \in G_{x}^{x}} f_{2}\left(\tilde{g}\left(h_{x}\right)^{-1}\right) \varphi_{x}\left(u_{\tilde{g}}\right) d \mu(x), \quad \text { from (i) and Lemma 5.3.8. }
\end{aligned}
$$


Then (5.39) holds. However, we already proved this is equivalent to equation (5.37). 


\section{Chapter 6}

\section{Renault-Deaconu Groupoid}

In this chapter we prove a theorem due to Thomsen [26] which characterizes the extremal KMS states on the full $\mathrm{C}^{*}$-algebra of the Renault-Deaconu groupoid. The definition of this groupoid depends on a local homeomorphism $\sigma: X \rightarrow X$ such that $\mathrm{X}$ has some topological properties. We can identify the subset of units with $X$.

We can apply Neshveyev's theorems to this groupoid $\mathrm{C}^{*}$-algebra in order to describe its KMS states. In this chapter we show that, on this groupoid, quasi-invariant measures are the same as conformal measures. Moreover, the corresponding measure of an extremal KMS state is either continuous or supported on an orbit.

Under certain conditions, Thomsen's theorem gives an explicit formula for the extremal KMS states. The results in this chapter are based on [9] and [26].

\subsection{Introduction}

Now we define the Renault-Deaconu groupoid and prove some of its topological properties.

Definition 6.1.1. Let $X$ be a locally compact second countable Hausdorff space. Let $\sigma$ : 
$X \rightarrow X$ be a local homeomorphism. The Renault-Deaconu groupoid is the groupoid

$$
\mathcal{G}=\left\{(x, k, y) \in X \times \mathbb{Z} \times X: \exists n, m \in \mathbb{N}, k=n-m, \sigma^{n}(x)=\sigma^{m}(y)\right\}
$$

such that

$$
\mathcal{G}^{(2)}=\left\{\left(\left(x_{1}, k_{1}, y_{1}\right),\left(x_{2}, k_{2}, y_{2}\right)\right) \in \mathcal{G} \times \mathcal{G}: y_{1}=x_{2}\right\}
$$

with the following multiplication and inversion laws

$$
\left(x, k_{1}, y\right)\left(y, k_{2}, z\right)=\left(x, k_{1}+k_{2}, z\right) \text { and }(x, k, y)^{-1}=(y,-k, x)
$$

and unit space defined by $\mathcal{G}^{(0)}=\{(x, 0, x): x \in X\}$.

The range and source maps are defined by $r(x, k, y)=(x, 0, x)$ and $s(x, k, y)=(y, 0, y)$. Since the map $x \mapsto(x, 0, x)$ is a bijection from $X$ to $\mathcal{G}^{(0)}$, we identify $X$ with $\mathcal{G}^{(0)}$.

Remark 6.1.2. We assume, by convention, that $\sigma^{0}$ is the identity.

The groupoid $\mathcal{G}$ can be understood intuitively as follows: given $x \in X$, we can interpret the sequence $\left\{\sigma^{n}(x)\right\}_{n \in \mathbb{N}}$ as a trajectory starting at $x$, as shown in Figure 6.1.

$$
x \longrightarrow \sigma(x) \longrightarrow \sigma^{1}(x) \longrightarrow \sigma^{2}(x) \longrightarrow \sigma^{3}(x) \longrightarrow \cdots
$$

Figure 6.1: The sequence $\left\{\sigma^{n}(x)\right\}_{n \in \mathbb{N}}, x \in X$, can be interpreted as the trajectory of $x$.

If, for some $y \in X$, there is some $n$ such that $\sigma^{n}(y)$ is an element of the trajectory of $x$, we can say the trajectories eventually meet. In other words, there exists $m$ such that $\sigma^{m}(x)=\sigma^{n}(y) . k=m-n$ is the delay of one trajectory with respect to the other. Hence 
$(x, k, y) \in \mathcal{G}$ if the trajectories of $x$ and $y$ eventually meet. This idea is shown in Figure 6.2.

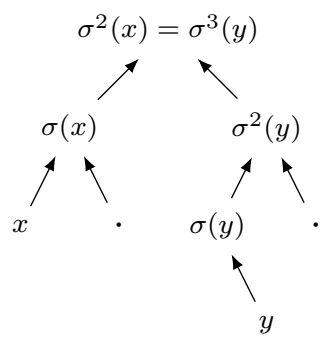

Figure 6.2: If $(x, k, y) \in \mathcal{G}$ then the trajectories $\left\{\sigma^{l}(x)\right\}_{l \in \mathbb{N}}$ and $\left\{\sigma^{l}(y)\right\}_{l \in \mathbb{N}}$ eventually meet. $k$ can be seen as the delay of one trajectory with respect to the other. In this figure, $k=-1$, since $\sigma^{2}(x)=\sigma^{3}(y)$.

Proposition 6.1.3. The Renault-Deaconu groupoid is a groupoid.

Proof. Let $\mathcal{G}$ be the Renault-Deaconu groupoid. Clearly the maps $r$ and $s$ are surjective.

The product is well-defined: In fact, let $g, h \in \mathcal{G}$ be composable. Then $g=(x, k, y)$, $h=(y, l, z)$ for some $x, y, z \in X, k, l \in \mathbb{Z}$.

By definition of $\mathcal{G}$, there exist $m, n \in \mathbb{N}$ such that $k=m-n$ and $\sigma^{m}(x)=\sigma^{n}(y)$. There are $p, q \in \mathbb{N}$ satisfying $l=p-q$ and $\sigma^{p}(y)=\sigma^{q}(z)$. Hence,

$$
\sigma^{m+p}(x)=\sigma^{p}\left(\sigma^{m}(x)\right)=\sigma^{p}\left(\sigma^{n}(y)\right)=\sigma^{p+n}(y)=\sigma^{n}\left(\sigma^{p}(y)\right)=\sigma^{n}\left(\sigma^{q}(z)\right)=\sigma^{n+q}(z) .
$$

The inverse is well-defined: Given $(x, k, y) \in \mathcal{G}$, there exist $m, n \in \mathbb{N}$ such that $k=m-n$ and $\sigma^{m}(x)=\sigma^{n}(y)$. Then $-k=n-m$ and $\sigma^{n}(y)=\sigma^{m}(x)$. Hence $(y,-k, x) \in \mathcal{G}$.

Now we show $\mathcal{G}$ satisfies properties (i)-(v) of Definition 3.1.1:

(i) Let $g=(x, k, y) \in \mathcal{G}, h=(y, l, z) \in \mathcal{G}$, then

$$
\begin{aligned}
& s(g h)=s(x, k+l, z)=z=s(h) \\
& r(g h)=r(x, k+l, z)=x=r(g) .
\end{aligned}
$$

(ii) Given $x \in X, r(x, 0, x)=(x, 0, x)=s(x, 0, x)$. 
(iii) Given $g=(x, k, y) \in \mathcal{G}$,

$$
\begin{aligned}
& g s(g)=(x, k, y)(y, 0, y)=(x, k, y)=g \\
& r(g) g=(x, 0, x)(x, k, y)=(x, k, y)=g .
\end{aligned}
$$

(iv) Let $\left(g_{1}, g_{2}\right),\left(g_{2}, g_{3}\right) \in \mathcal{G}^{(2)}$. Then $g_{1}=\left(x, k_{1}, y\right), g_{2}=\left(y, k_{2}, z\right), g_{3}=\left(z, k_{3}, w\right)$. Hence,

$$
\begin{aligned}
& \left(g_{1} g_{2}\right) g_{3}=\left(x, k_{1}+k_{2}, z\right)\left(z, k_{3}, w\right)=\left(x, k_{1}+k_{2}+k_{3}, w\right) \\
& g_{1}\left(g_{2} g_{3}\right)=\left(x, k_{1}, y\right)\left(y, k_{2}+k_{3}, w\right)=\left(x, k_{1}+k_{2}+k_{3}, w\right) .
\end{aligned}
$$

(v) Given $g=(x, k, y) \in \mathcal{G}$,

$$
\begin{aligned}
& g g^{-1}=(x, k, y)(y,-k, x)=(x, 0, x)=r(g) \\
& g^{-1} g=(y,-k, x)(x, k, y)=(y, 0, y)=s(g) .
\end{aligned}
$$

Example 6.1.4. Let $X=\left\{x=\left\{x_{n}\right\}_{n \in \mathbb{N}}: x_{n} \in\{0,1\}\right\}$. Endow $X$ with the metric

$$
d(x, y)=2^{-\min \left\{n \in \mathbb{N}: x_{n} \neq y_{n}\right\}} .
$$

Moreover, $\sigma$ is a local homeomorphism. Indeed, let $x \in X$. Define the map $\rho: X \rightarrow X$ by

$$
\rho(y)_{n}= \begin{cases}x_{0}, & \text { if } n=0 \\ y_{n-1} & \text { if } n \geq 1\end{cases}
$$

Then $\rho$ is continuous, since for $y, z \in X$,

$$
d(\sigma(y), \sigma(z))=2^{-\min \left\{n \geq 1: y_{n-1} \neq z_{n-1}\right\}}=2^{-1} d(y, z)
$$


Moreover, for every $y \in B(x, 1), y_{0}=x_{0}$. Hence $y=\rho \circ \sigma(y)=\sigma \circ \rho(y)$. Therefore $\sigma$ is invertible on $B(x, 1)$ with inverse $\rho$. That is, $\rho$ is a local homeomorphism.

Then $(x, k, y) \in \mathcal{G}$ if there are $n, m \in \mathbb{N}$ such that $k=n-m$ and $\sigma^{n}(x)=\sigma^{m}(y)$. For instance, if $x=(0,1,0,0, \ldots)$ and $y=(1,1,1,1,0,0, \ldots)$ then $(x,-2, y) \in \mathcal{G}$, as shown in Figure 6.3.

$$
\begin{aligned}
& x=\left(0, \frac{\sigma(x)}{1,0,0, \ldots)}\right. \\
& y=\left(1,1,1, \frac{1,0,0, \ldots)}{\sigma^{3}(y)}\right.
\end{aligned}
$$

Figure 6.3: It follows from the equality $\sigma(x)=\sigma^{3}(y)=(1,0,0, \ldots)$ that $(x,-2, y) \in \mathcal{G}$.

Now we define a topology on $\mathcal{G}$ which makes the Renault-Deaconu groupoid an étale groupoid.

Given $A, B$ open subsets of $X, m, n \in \mathbb{N}$, let

$$
\mathcal{U}_{A, B}^{n, m}=\left\{(x, n-m, y) \in \mathcal{G}: \sigma^{n}(x)=\sigma^{m}(y), x \in A, y \in B\right\}
$$

These sets form a basis of the topology on $\mathcal{G}$.

Proposition 6.1.5. The family of sets $\mathcal{U}_{A, B}^{n, m}$, for $A, B$ open subsets of $X$ and $n, m \in \mathbb{N}$, generates a topology on $\mathcal{G}$. Moreover, $\mathcal{G}$ is second countable.

Proof. First we show that $\mathcal{G}$ is the union of these sets. Let $(x, k, y) \in \mathcal{G}$. Then there exist $n, m$ such that $\sigma^{n}(x)=\sigma^{m}(y)$. Hence $(x, n-m, y) \in \mathcal{U}_{X, X}^{n, m}$.

Now we prove that for every $(x, k, y) \in \mathcal{U}_{A_{1}, B_{1}}^{n_{1}, m_{1}} \cap \mathcal{U}_{A_{2}, B_{2}}^{n_{2}, m_{2}}$ there exists $\mathcal{U}_{A, B}^{n, m}$ such that $(x, k, y) \in \mathcal{U}_{A, B}^{n, m} \subset \mathcal{U}_{A_{1}, B_{1}}^{n_{1}, m_{1}} \cap \mathcal{U}_{A_{2}, B_{2}}^{n_{2}, m_{2}}$.

Let $(x, k, y) \in \mathcal{U}_{A_{1}, B_{1}}^{n_{1}, m_{1}} \cap \mathcal{U}_{A_{2}, B_{2}}^{n_{2}, m_{2}}$. Then,

$$
x \in A_{1} \cap A_{2}, \quad y \in B_{1} \cap B_{2}, \quad \sigma^{n_{1}}(x)=\sigma^{m_{1}}(y), \quad \sigma^{n_{2}}(x)=\sigma^{m_{2}}(y) .
$$


Let $p_{1}=n_{2}, p_{2}=n_{1}$. Note that $m_{1}-n_{1}=m_{2}-n_{2}$ implies $m_{1}+p_{1}=m_{2}+p_{2}$. From the definition of $p_{1}, p_{2}$, we have $n_{1}+p_{1}=n_{2}+p_{2}$.

For $i=1,2$, let $U_{i}$ be an open neighborhood of $\sigma^{n_{i}}(x)$ such that $\sigma^{p_{i}}$ is injective on $U_{i}$. Let

$$
\begin{aligned}
& n=n_{1}+p_{1}=n_{2}+p_{2} \\
& m=m_{1}+p_{1}=m_{2}+p_{2} \\
& A=A_{1} \cap A_{2} \cap \sigma^{-n_{1}}\left(U_{1}\right) \cap \sigma^{-n_{2}}\left(U_{2}\right) \\
& B=B_{1} \cap B_{2} \cap \sigma^{-m_{1}}\left(U_{1}\right) \cap \sigma^{-m_{2}}\left(U_{2}\right) .
\end{aligned}
$$

From continuity of $\sigma^{n_{i}}, \sigma^{m_{i}}$, it follows that $A$ and $B$ are open sets.

We show that $(x, k, y) \in \mathcal{U}_{A, B}^{n, m}$. Clearly $n-m=n_{1}+p_{1}-\left(m_{1}+p_{1}\right)=n_{1}-m_{1}=k$. Then,

$$
\sigma^{n}(x)=\sigma^{p_{1}+n_{1}}(x)=\sigma^{p_{1}}\left(\sigma^{n_{1}}(x)\right)=\sigma^{p_{1}}\left(\sigma^{m_{1}}(y)\right)=\sigma^{p_{1}+m_{1}}(y)=\sigma^{m}(y) .
$$

For $i=1,2, \sigma^{n_{i}}(x) \in U_{i}, \sigma^{m_{i}}(y)=\sigma^{n_{i}}(x) \in U_{i}$. Then $x \in A, y \in B$. Therefore $(x, k, y) \in$ $\mathcal{U}_{A, B}^{n, m}$

Now we show that $\mathcal{U}_{A, B}^{n, m} \subset \mathcal{U}_{A_{1}, B_{1}}^{n_{1}, m_{1}} \cap \mathcal{U}_{A_{2}, B_{2}}^{n_{2}, m_{2}}$. Let $(u, k, v) \in \mathcal{U}_{A, B}^{n, m}$.

For $i=1,2$, we have

$$
\begin{aligned}
\sigma^{n}(u) & =\sigma^{m}(v) \\
\sigma^{n_{i}+p_{i}}(u) & =\sigma^{m_{i}+p_{i}}(v) \\
\sigma^{p_{i}}\left(\sigma^{n_{i}}(u)\right) & =\sigma^{p_{i}}\left(\sigma^{m_{i}}(u)\right) .
\end{aligned}
$$

Since $\sigma^{n_{i}}(u), \sigma^{m_{i}}(v) \in U$ and $\sigma^{p_{i}}$ is injective on this set, it follows that $\sigma^{n_{i}}(u)=\sigma^{m_{i}}(v)$. Hence $(u, k, v) \in \mathcal{U}_{A_{i}, B_{i}}^{n_{i}, m_{i}}$. Therefore $(u, k, v) \in \mathcal{U}_{A_{1}, B_{1}}^{n_{1}, m_{1}} \cap \mathcal{U}_{A_{2}, B_{2}}^{n_{2}, m_{2}}$.

Now we show that $\mathcal{G}$ is second countable. 
Let $\mathcal{B}$ be a countable base of $X$. Then the family of sets of the form

$$
\mathcal{U}_{A, B}^{n, m} \text { such that } n, m \in \mathbb{N} \text { and } A, B \in \mathcal{B}
$$

is also countable. We show this family form a base for $\mathcal{G}$. Let $\mathcal{U}_{A, B}^{n, m}$ with $A, B$ arbitrary open sets in $X$ and $n, m \in \mathbb{N}$. Let $(x, n-m, y) \in \mathcal{U}_{A, B}^{n, m}$. There exists $\tilde{A}, \tilde{B}$ such that $x \in \tilde{A} \subset A$, $y \in \tilde{B} \subset B$. Then $(x, n-m, y) \in \mathcal{U}_{\tilde{A}, \tilde{B}}^{n, m} \subset \mathcal{U}_{A, B}^{n, m}$.

Lemma 6.1.6. Let $\left\{\left(x_{i}, k_{i}, y_{i}\right)\right\}_{i \in \mathbb{N}}$ be a sequence in $\mathcal{G}$ converging to $(x, k, y)$. Then $x_{i} \rightarrow x$, $y_{i} \rightarrow y$ and there exists $i_{0}$ such that $k_{i}=k$ for every $i \geq i_{0}$. Hence we can assume, without loss of generality, that $k_{i}$ is constant.

Proof. Let $n, m \in \mathbb{N}$ such that $\sigma^{n}(x)=\sigma^{m}(y)$ and $k=n-m$. Let $A, B$ be neighborhoods of $x, y$, respectively. Then there exists $i_{0}$ such that for every $i \geq i_{0},\left(x_{i}, k_{i}, y_{i}\right) \in \mathcal{U}_{A, B}^{n, m}$, then,

$$
x \in A, \quad y \in B, \quad k_{i}=n-m=k .
$$

Therefore $k_{i}$ is eventually constant, $x_{i} \rightarrow x$ and $y_{i} \rightarrow y$.

Lemma 6.1.7. Fix $n_{0}, m_{0} \in \mathbb{N}$. Given a sequence $\left\{\left(x_{i}, k, y_{i}\right)\right\}_{i \in \mathbb{N}}$ a net assume that for all neighborhoods $A$ of $x$ and $B$ of $y$ there exists $i_{0}$ such that $\left(x_{i}, k, y_{i}\right) \in \mathcal{U}_{A, B}^{n_{0}, m_{0}}$ for $i \geq i_{0}$.

Then for every $A, B$ open neighborhoods of $x, y$, respectively, $n, m$ such that $(x, k, y) \in$ $\mathcal{U}_{A, B}^{n, m}$. There exists $i_{0}$ such that

$$
\left(x_{i}, k, y_{i}\right) \in \mathcal{U}_{A, B}^{n, m} \quad \text { for } i \geq i_{0}
$$

Then $\left(x_{i}, k, y_{i}\right) \rightarrow(x, k, y)$.

Proof. Note that $k=n_{0}-m_{0}$. Let $n, m \in \mathbb{N}$ such that $\sigma^{n}(x)=\sigma^{m}(y)$ and $k=n-m$. Then $n_{0}+m=n+m_{0}$.

Let $V$ be an open neighborhood of $\sigma^{n}(x)=\sigma^{m}(y)$ where $\sigma^{n_{0}}$ and is injective. Since $x_{i} \rightarrow x$ and $y_{i} \rightarrow y$ and $\sigma$ is continuous, there exists $i_{0}$ such that for every $i \geq i_{0}, \sigma^{n}\left(x_{i}\right), \sigma^{m}\left(y_{i}\right) \in V$. 
Then, given $i \geq i_{0}$,

$$
\sigma^{n+n_{0}}\left(x_{i}\right)=\sigma^{n}\left(\sigma^{n_{0}}\left(x_{i}\right)\right)=\sigma^{n}\left(\sigma^{m_{0}}\left(y_{i}\right)\right)=\sigma^{n+m_{0}}\left(y_{i}\right)=\sigma^{n_{0}+m}\left(y_{i}\right) .
$$

Then $\sigma^{n_{0}}\left(\sigma^{n}\left(x_{i}\right)\right)=\sigma^{n_{0}}\left(\sigma^{m}\left(y_{i}\right)\right)$. Since $\sigma^{n}\left(x_{i}\right), \sigma^{m}\left(y_{i}\right) \in V$ and $\sigma^{n_{0}}$ is injective on $V$, it follows that $\sigma^{n}\left(x_{i}\right)=\sigma^{m}\left(y_{i}\right)$ for every $i \geq i_{0}$.

Note that $(x, k, y) \notin \mathcal{U}_{A, B}^{n, m}$ if $n-m \neq k$.

Let $A, B$ be open neighborhoods of $x, y$ respectively. Let $m, n$ such that $m-n=k$. Since $x_{i} \rightarrow x$ and $y_{i} \rightarrow y$, there exists $i_{0}$ such that if $i \geq i_{0}$,

$$
x_{i} \in A, \quad y_{i} \in B, \quad \sigma^{n}\left(x_{i}\right)=\sigma^{m}\left(y_{i}\right),
$$

or equivalently, $\left(x_{i}, k, y_{i}\right) \in \mathcal{U}_{A, B}^{n, m}$ for every $i \geq i_{0}$.

Corollary 6.1.8. Let $(x, n-m, y) \in \mathcal{G}$. Let $\left\{x_{i}\right\}_{i \in \mathbb{N}},\left\{y_{i}\right\}_{i \in \mathbb{N}}$ be sequences in $X$ such that $x_{i} \rightarrow x$ and $y_{i} \rightarrow y$. If $\sigma^{n}\left(x_{i}\right)=\sigma^{m}\left(y_{i}\right)$ for each $i$, then $\left(x_{i}, n-m, y_{i}\right) \rightarrow(x, n-m, y)$ in $\mathcal{G}$.

Proof. Let $A$ be an open neighborhood of $x, B$ an open neighborhood of $y$. There exists $i_{0}$ such that, for every $i \geq i_{0}, x_{i} \in A, y_{i} \in B$. By hypothesis, $\sigma^{n}\left(x_{i}\right)=\sigma^{m}\left(y_{i}\right)$. Then $\left(x_{i}, n-m, y_{i}\right) \in \mathcal{U}_{A, B}^{n, m}$. From Lemma 6.1.7, it follows that $\left(x_{i}, n-m, y_{i}\right) \rightarrow(x, n-m, y)$ in $\mathcal{G}$.

Theorem 6.1.9. The Renault-Deaconu groupoid $\mathcal{G}$, with topology generated by $\mathcal{U}_{A, B}^{n, m}$ is a topological groupoid, locally compact Hausdorff, second countable and étale.

Proof. $\quad \bullet \mathcal{G}$ is a topological groupoid

(i) $\mathcal{G}^{(2)}$ is closed in $\mathcal{G} \times \mathcal{G}$.

Assume $\left\{\left(g_{i}, h_{i}\right)\right\}_{i \in \mathbb{N}}$ is a sequence in $\mathcal{G}^{(2)}$ converging to $(g, h) \in \mathcal{G} \times \mathcal{G}$. Then $g_{i}=\left(x_{i}, k_{i}, y_{i}\right), h_{i}=\left(y_{i}, l_{i}, z_{i}\right)$ for each $i$. Assume $g=(x, k, y)$ and $h=(\tilde{y}, l, z)$. It follows from Lemma 6.1.6 that $y_{i} \rightarrow y$ and $y_{i} \rightarrow \tilde{y}$ in $X$. Since $X$ is Hausdorff, we have $y=\tilde{y}$. Therefore $(g, h) \in \mathcal{G}^{(2)}$. 
(ii) The inverse is continuous

Denote the inverse map by $\iota$. Let $\mathcal{U}_{B, A}^{m, n}$ be an open set in the topological base of $\mathcal{G}$. Then,

$$
\begin{aligned}
\iota^{-1}\left(\mathcal{U}_{B, A}^{m, n}\right) & =\left\{(x, k, y):(y,-k, x) \in \mathcal{U}_{B, A}^{m, n}\right\} \\
& =\left\{(x, k, y): y \in B, x \in A, \sigma^{m}(y)=\sigma^{n}(x), k=n-m\right\} \\
& =\mathcal{U}_{A, B}^{n, m} .
\end{aligned}
$$

Therefore the inverse map is continuous.

(iii) The product is continuous

Let $\left(g_{i}, h_{i}\right) \rightarrow(g, h)$ in $\mathcal{G}^{(2)}$. We can assume $g=(x, k, y), h=(y, l, z)$ and for each $i, g_{i}=\left(x_{i}, k, y_{i}\right)$ and $h_{i}=\left(y_{i}, l, z_{i}\right)$.

Let $n_{1}, m_{1}, n_{2}, m_{2} \in \mathbb{N}$ such that

$$
\begin{array}{ll}
k=n_{1}-m_{1}, & \sigma^{n_{1}}(x)=\sigma^{m_{1}}(y) \\
l=n_{2}-m_{2}, & \sigma^{n_{2}}(y)=\sigma^{m_{2}}(z) .
\end{array}
$$

Since $\left(x_{i}, k, y_{i}\right) \rightarrow(x, k, y)$ and $\left(y_{i}, l, z_{i}\right) \rightarrow(y, l, z)$, it follows that for $A, B$ neighborhoods of $x$ and $z$, there exists $i_{0}$ such that

$$
\left(x_{i}, k, y_{i}\right) \in \mathcal{U}_{A, X}^{n_{1}, m_{1}} \quad \text { and } \quad\left(y_{i}, k, z_{i}\right) \in \mathcal{U}_{X, B}^{n_{1}, m_{1}} \quad \text { for } \quad i \geq i_{0}
$$

Hence, if $i \geq i_{0}$,

$$
\begin{aligned}
\sigma^{n_{1}+n_{2}}\left(x_{i}\right) & =\sigma^{n_{2}}\left(\sigma^{n_{1}}\left(x_{i}\right)\right)=\sigma^{n_{2}}\left(\sigma^{m_{1}}\left(y_{i}\right)\right)=\sigma^{n_{2}+m_{1}}\left(y_{i}\right) \\
& =\sigma^{m_{1}}\left(\sigma^{n_{2}}\left(y_{i}\right)\right)=\sigma^{m_{1}}\left(\sigma^{m_{2}}\left(z_{i}\right)\right)=\sigma^{m_{1}+m_{2}}\left(z_{i}\right) .
\end{aligned}
$$


Then $\left(x_{i}, k+l, z_{i}\right) \in \mathcal{U}_{A, B}^{n_{1}+n_{2}, m_{1}+m_{2}}$. It follows from Lemma 6.1 .7 that

$$
\left(x_{i}, k+l, z_{i}\right) \rightarrow(x, k+l, z)
$$

- $\mathcal{G}$ is Hausdorff

Let $g_{i}=\left(x_{i}, n_{i}-m_{i}, y_{i}\right) \in \mathcal{G}$ such that $\sigma^{n_{i}}\left(x_{i}\right)=\sigma^{m_{i}}\left(y_{i}\right), i=1,2$. Assume $g_{1} \neq g_{2}$.

(i) If $n_{1}-m_{1} \neq n_{2}-m_{2}$, then $g_{1} \in \mathcal{U}_{X, X}^{n_{1}, m_{1}}, g_{2} \in \mathcal{U}_{X, X}^{n_{2}, m_{2}}$ and $\mathcal{U}_{X, X}^{n_{1}, m_{1}} \cap \mathcal{U}_{X, X}^{n_{2}, m_{2}}=\varnothing$.

(ii) If $n_{1}-m_{1}=n_{2}-m_{2}$, Then $x_{1} \neq x_{2}$ or $y_{1} \neq y_{2}$. Assume $x_{1} \neq x_{2}$. Since $X$ is Hausdorff, we can choose $A_{1}, A_{2}$ open neighborhoods of $x_{1}, x_{2}$ respectively, such that $A_{1} \cap A_{2}=\varnothing$. Then $g_{i} \in \mathcal{U}_{A_{i}, X}^{n_{i}, m_{i}}, i=1,2$, and $\mathcal{U}_{A_{1}, X}^{n_{1}, m_{1}} \cap \mathcal{U}_{A_{2}, X}^{n_{2}, m_{2}}=\varnothing$.

The proof for $y_{1} \neq y_{2}$ is analogous.

- $\mathcal{G}$ is locally compact.

Let $A, B$ be open sets of $X$ such that $\bar{A}, \bar{B}$ are compact, and let $n, m \in \mathbb{N}$. Then $\mathcal{U}_{A, B}^{n, m} \subset \mathcal{U}_{\bar{A}, \bar{B}}^{n, m}$, where

$$
\mathcal{U}_{\bar{A}, \bar{B}}=\left\{(x, n-m, y) \in \mathcal{G}: \sigma^{n}(x)=\sigma^{m}(y), x \in \bar{A}, y \in \bar{B}\right\}
$$

Let $\left\{\left(x_{i}, n-m, y_{i}\right)\right\}_{i \in \mathbb{N}}$ be a sequence in $\mathcal{U}_{\bar{A}, \bar{B}}$. Then $\left\{\left(x_{i}, y_{i}\right)\right\}_{i \in \mathbb{N}}$ is a sequence in the compact set $\overline{A \times B}$. Then there exists a subsequence $\left\{\left(x_{i_{j}}, y_{i_{j}}\right)\right\}_{j \in \mathbb{N}}$ such that $x_{i_{j}} \rightarrow x$ for some $x \in \bar{A}$ and $y_{i_{j}} \rightarrow y$ for some $y \in \bar{B}$. By continuity of $\sigma, \sigma^{n}(x)=\sigma^{m}(y)$. Therefore $\mathcal{U}_{\bar{A}, \bar{B}}$ is compact.

- $\mathcal{G}$ is étale.

Let $(x, n-m, y) \in \mathcal{G}$ such that $\sigma^{n}(x)=\sigma^{m}(y)$. Since $\sigma$ is a local homeomorphism, 
there are $A, B$ open neighborhoods of $x, y$, respectively, satisfying

$$
\begin{gathered}
\sigma^{n}(A) \text { is open and }\left.\sigma^{n}\right|_{A}: A \rightarrow \sigma^{n}(A) \text { is a homeomorphism } \\
\sigma^{m}(B) \text { is open and }\left.\sigma^{m}\right|_{B}: B \rightarrow \sigma^{m}(B) \text { is a homeomorphism. }
\end{gathered}
$$

Then $(x, n-m, y) \in \mathcal{U}_{A, B}^{n, m}$. Since $\mathcal{G}$ is a topological groupoid, $r$ is continuous. In order to prove $r$ is a local homeomorphism, we will show $r$ is injective on $\mathcal{U}_{A, B}^{n, m}, r\left(\mathcal{U}_{A, B}^{n, m}\right)$ is open and $\left.r\right|_{\mathcal{U}_{A, B}^{n, m}} ^{-1}$ is continuous.

(i) $r$ is injective.

Suppose there exist $x_{1}, x_{2} \in A, y_{1}, y_{2} \in B$ such that $r\left(x_{1}, n-m, y_{1}\right)=r\left(x_{2}, n-\right.$ $\left.m, y_{2}\right)$. Then $y_{1}=y_{2}$. Moreover,

$$
x_{1}=\left.\sigma^{-n}\right|_{A}\left(\sigma^{m}\left(y_{1}\right)\right)=\left.\sigma^{-n}\right|_{A}\left(\sigma^{m}\left(y_{2}\right)\right)=x_{2}
$$

Therefore $\left(x_{1}, n-m, y_{1}\right)=\left(x_{2}, n-m, y_{2}\right)$.

(ii) $r\left(\mathcal{U}_{A, B}^{n, m}\right)$ is open.

$$
\begin{aligned}
r\left(\mathcal{U}_{A, B}^{n, m}\right) & =\left\{(y, 0, y) \in \mathcal{G}:(x, n-m, y) \in \mathcal{U}_{A, B}^{n, m}\right\} \\
& =\left\{(y, 0, y) \in \mathcal{G}: x \in A, y \in B, \sigma^{n}(x)=\sigma^{m}(y)\right\} \\
& =\left\{(y, 0, y) \in \mathcal{G}: y \in B, x=\left.\sigma\right|_{A} ^{-n}\left(\sigma^{m}(y)\right) \in A\right\} \\
& =\left\{(y, 0, y) \in \mathcal{G}: y \in B, \sigma^{m}(y) \in \sigma^{n}(A)\right\} \quad \text { note that } \sigma^{n}(A) \text { is open } \\
& =\left\{(y, 0, y) \in \mathcal{G}: y \in B,\left.y \in \sigma\right|_{B} ^{-m}\left(\sigma^{n}(A)\right)\right\} \\
& =\mathcal{U}_{C, C}^{0,0},
\end{aligned}
$$

where $C=\left.B \cap \sigma\right|_{B} ^{-m}\left(\sigma^{n}(A)\right)$.

(iii) $\left.r\right|_{\mathcal{U}_{A, B}^{n, m}} ^{-1}$ is continuous.

Let $\left\{\left(y_{i}, 0, y_{i}\right)\right\}_{i \in \mathbb{N}}$ be a sequence in $r\left(\mathcal{U}_{A, B}^{n, m}\right)$ converging to some $(y, 0, y)$ in $r\left(\mathcal{U}_{A, B}^{n, m}\right)$. Then $y_{i} \rightarrow y \in B$. Define the sequence $x_{i}=\left.\sigma\right|_{A} ^{-n}\left(\sigma^{m}\left(y_{i}\right)\right)$. Then $x_{i} \rightarrow x=$ 
$\left.\sigma\right|_{A} ^{-n}\left(\sigma^{m}(y)\right)$

Note that $x_{i}$ is the only element in $A$ satisfying $\sigma^{n}\left(x_{i}\right)=\sigma^{m}\left(y_{i}\right)$, hence,

$$
\left(x_{i}, n-m, y_{i}\right)=\left.r\right|_{\mathcal{U}_{A, B}^{n, m}} ^{-1}\left(y_{i}, 0, y_{i}\right) .
$$

Analogously, $(x, n-m, y)=\left.r\right|_{\mathcal{U}_{A, B}^{n, m}} ^{-1}(y, 0, y)$. Then, it follows from Corollary 6.1.8 that $\left(x_{i}, n-m, y_{i}\right) \rightarrow(x, n-m, y)$.

Analogously, we can show $s$ is a local homeomorphism.

The next lemma shows that we can identify $X$ wth the unit space of $\mathcal{G}$. In this chapter, we fix $X$ and $\sigma$, and we assume $\mathcal{G}$ is the Renault-Deaconu groupoid.

Lemma 6.1.10. We can identify the unit space $\mathcal{G}^{(0)}$ with the set $X$. In fact, both have the same topology.

Proof. Clearly the map $\iota: \mathcal{G}^{(0)} \rightarrow X$ defined by $(x, 0, x) \rightarrow x$ is a bijection. Denote this map by $\iota$. We will show $\iota$ is a homeomorphism.

Let $n, m \in \mathbb{N}, A, B \subset X$ open sets. If $\mathcal{U}_{A, B}^{n, m} \cap \mathcal{G}^{(0)} \neq \varnothing$, then $n=m$. In this case, $\mathcal{U}_{A, B}^{n, m} \subset \mathcal{G}^{(0)}$. Hence,

$$
\iota\left(\mathcal{U}_{A, B}^{n, m}\right)=\iota(\{(x, 0, x): x \in A \cap B\})=A \cap B
$$

is open in $X$. On the other hand, for any open set $A \subset X$,

$$
\iota^{-1}(A)=\{(x, 0, x): x \in A\}=\mathcal{U}_{A, A}^{0,0},
$$

Therefore $\iota$ is a homeomorphism. 


\section{$6.2 \quad$ Full orbits}

Given $x \in X$, the full orbit of $x$ denotes the set of elements in $X$ whose trajectories eventually meet the trajectory of $x$. There are two types of orbits, periodic and aperiodic. In this section we will study their properties.

Definition 6.2.1. Let $x \in X$, the full orbit of $x$ is the set

$$
\mathcal{O}(x)=\{y \in X: \text { there exists } k \in \mathbb{Z} \text { such that }(x, k, y) \in \mathcal{G}\}
$$

Lemma 6.2.2. Given $x, y \in X, \mathcal{O}(x)=\mathcal{O}(y)$ if, and only if, $y \in \mathcal{O}(x)$.

Proof. Let $y \in \mathcal{O}(x)$. There exists $(x, k, y) \in \mathcal{G}$.

- $\mathcal{O}(y) \subset \mathcal{O}(y)$ Let $z \in \mathcal{O}(y)$. There exists $(y, l, z) \in \mathcal{G}$. Hence $(x, k+l, z) \in \mathcal{G}$. Then $z \in \mathcal{O}(x)$. Therefore $\mathcal{O}(y) \subset \mathcal{O}(x)$.

- $\mathcal{O}(x) \subset \mathcal{O}(y)$ Let $z \in \mathcal{O}(x)$. There exists $(x, l, z) \in \mathcal{G}$. Then $(z, k-l, y) \in \mathcal{G}$. Thus $z \in \mathcal{O}(y)$. Then $\mathcal{O}(x) \subset \mathcal{O}(y)$.

Therefore $\mathcal{O}(x)=\mathcal{O}(y)$.

Conversely, assume $\mathcal{O}(x)=\mathcal{O}(y)$. By definition, $(y, 0, y) \in \mathcal{G}$. Then $y \in \mathcal{O}(y)=\mathcal{O}(x)$.

Remark 6.2.3. Lemma 6.2 .2 is a fact of general groupoids. Given a groupoid $G$, if we define the set $\mathcal{O}(x)=r\left(G_{x}\right)$ for every $x \in G^{(0)}$, then the Lemma 6.2.2 holds.

Lemma 6.2.4. Given $x \in X, \mathcal{O}(x)$ is countable.

Proof.

$$
\begin{aligned}
\mathcal{O}(x) & =\{y \in X: \text { there exists } k \in \mathbb{Z} \text { such that }(x, k, y) \in \mathcal{G}\} \\
& =\left\{y \in X: \mathcal{G}_{x}^{y} \neq \varnothing\right\}
\end{aligned}
$$




$$
\begin{aligned}
& =\left\{r(g): g \in \mathcal{G}_{x} \neq \varnothing\right\} \quad \text { identifying } X \text { with } \mathcal{G}^{(0)} \\
& =r\left(\mathcal{G}_{x}\right) .
\end{aligned}
$$

$\mathcal{G}$ is second countable étale from Theorem 6.1.9. It follows from Proposition 3.2.11 that $\mathcal{G}_{x}$ is countable. Therefore $\mathcal{O}(x)$ is countable.

There are two types of full orbits: periodic and aperiodic orbits. This difference will be fundamental when we define the extremal conformal measures later.

Definition 6.2.5. Given $x \in X$, we say it is periodic or $\sigma$-periodic if there is a positive integer $p$ such that

$$
\sigma^{p}(x)=x
$$

The minimum positive natural number such that (6.1) holds is called the minimal period of $x$.

Definition 6.2.6. A point $z \in X$ is called aperiodic if $\mathcal{O}(z)$ does not contain periodic points.

Definition 6.2.7. Let $y \in X$. If $\mathcal{O}(y)$ has periodic points, $\mathcal{O}(y)$ is called periodic. Otherwise, $\mathcal{O}(y)$ is aperiodic.

When represented graphically, periodic and aperiodic orbits look different. An aperiodic orbits look like a tree while a periodic orbits has a single cycle, the trajectory of a periodic 
element. Figure 6.4 shows these two types of orbits.

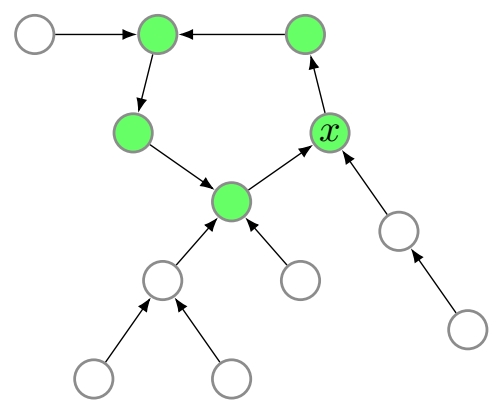

(a)

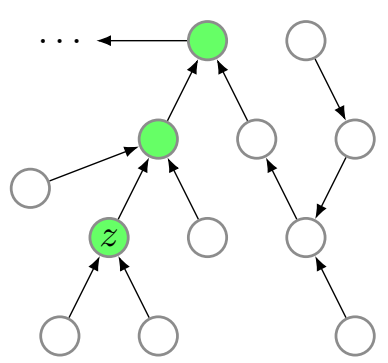

(b)

Figure 6.4: Periodic and aperiodic orbits look different. The periodic orbit (a) has a cycle, while the aperiodic orbit looks like a tree. Circles in green represent the trajectories of $x$ and $z$.

Given $y$ in the orbit of $x$, the trajectory of $y$ eventually meets the trajectory of $x$. If $x$ is periodic, we can see in Figure 6.4 that $y$ eventually meets the point $x$, that is, there exists an $n$ such that $\sigma^{n}(y)=x$. Now we will prove this result.

Lemma 6.2.8. Let $x \in X$ be a periodic point with minimum period $p$. Given $y \in \mathcal{O}(x)$, there exists $n \in \mathbb{N}$ such that $\sigma^{n}(y)=x$.

Proof. Let $y \in \mathcal{O}(x)$. Let $n_{1}, m_{1} \in \mathbb{N}$ such that $\sigma^{n_{1}}(x)=\sigma^{m_{1}}(x)$. Let $N$ be a natural number such that $N+m_{1} \in p \mathbb{N}$. Define $n=N+n_{1}$. Then

$$
\sigma^{n}(y)=\sigma^{N+n_{1}}(y)=\sigma^{N+m_{1}}(x)=x .
$$

Lemma 6.2.9. Assume the set of periodic points in $X$ is countable. Let $I$ denote the set of aperiodic points. Then $I$ is countable

Proof. Let $N$ be the set of points with periodic orbits. Then $I=X \backslash N$ and we can write

$$
N=\bigcup_{\substack{x \in X \\ x \text { periodic }}} \mathcal{O}(x)
$$


$\mathcal{O}(x)$ is countable for every $x \in X$ by Lemma 6.2.4. Moreover, the set of periodic points is countable by hypothesis. Then $N$ is countable and $\mu(N)=0$.

Lemma 6.2.10. Let $z \in X$ be aperiodic. Then $\mathcal{G}_{z}^{z}=\{(z, 0, z)\}$. If $y \in X$ is such that $\mathcal{O}(y)$ has a periodic point with minimum period $p$, then $\mathcal{G}_{y}^{y}=\{(y, k p, y): k \in \mathbb{Z}\}$.

Proof. Let $z \in X$ be aperiodic. Let $k \in \mathbb{Z}$ such that $(z, k, z) \in \mathcal{G}$. Then there exists $n, m \in \mathbb{N}$ such that $\sigma^{n}(z)=\sigma^{m}(z), k=n-m$.

Assume without loss of generality that $n>m$. Denote $x=\sigma^{m}(z) \in \mathcal{O}(z)$. Then $x$ is periodic, since

$$
\sigma^{n-m}(x)=\sigma^{n-m}\left(\sigma^{m}(z)\right)=\sigma^{n}(z)=\sigma^{m}(z)=x .
$$

Contradiction. Then $k=0$ and therefore $\mathcal{G}_{z}^{z}=\{(z, 0, z)\}$.

Let $y \in X$ such that $\mathcal{O}(y)$ is periodic. Then there exist $x \in X$ periodic with minimum period $p$, and $l \in \mathbb{Z}$ such that $(x, l, y) \in \mathcal{G}$. Then $(y,-l, x)(x, p, x)(x, l, p)=(y, p, y) \in \mathcal{G}$. Therefore, by induction, $(y, k p, y) \in \mathcal{G}$ for every $k \in \mathbb{Z}$.

Suppose there exists $k \notin p \mathbb{Z}$ such that $(y, k, y) \in \mathcal{G}_{y}^{y}$. Let $k_{1}, k_{2} \in \mathbb{N}$ such that $k=k_{1}+k_{2} p$, $0 \leq k_{1}<p$. Note that $k_{1} \neq 0$ by hypothesis.

Then $(x, k, x) \in \mathcal{G}$ since $(x, k, x)=(y,-l, x)(y, k, y)(y, l, x)$. Hence there exist $n, m \in \mathbb{N}$ such that $n-m=k$ and $\sigma^{n}(x)=\sigma^{m}(x)$.

Assume $n>m$ without loss of generality. Let $N$ be an integer such that $N+m \in p \mathbb{N}$. Then,

$$
\begin{aligned}
x & =\sigma^{m+N}(x)=\sigma^{n+N}(x)=\sigma^{n-m}\left(\sigma^{m+N}(x)\right)=\sigma^{n-m}(x) \\
& =\sigma^{k}(x)=\sigma^{k_{1}+k_{2} p}(x)=\sigma^{k_{1}}\left(\sigma^{k_{2} p}(x)\right)=\sigma^{k_{1}}(x) .
\end{aligned}
$$

Contradiction, since $x$ has minimum period $p$. Therefore $\mathcal{G}_{y}^{y}=\{(y, k p, y): k \in \mathbb{Z}\}$. 


\subsection{Conformal Measures}

We will show explicitly all the extremal atomic $e^{\beta F}$-conformal probability measures on $\mathcal{G}$. for $\beta \neq 0$.

Now we define conformal measures as described in [8].

Definition 6.3.1. Consider a measurable function $T: X \rightarrow X$ on a measurable space $(X, \mathcal{F})$ and a measurable nonnegative function $f$ on $X$. A measure $\mu$ on $(X, \mathcal{F})$ is called $f$-conformal if

$$
\mu(T(A))=\int_{A} f(x) d \mu(x)
$$

whenever $A \subset X$ is a measurable set, for which $T(A)$ is measurable and $T: A \rightarrow T(A)$ is invertible.

A set $A$ as in Definition 6.3.1 is called special.

The set of $f$-conformal probability measures $\mu$ forms a convex set. We say $\mu$ is extremal if $\mu$ is an extremal point in this set.

Definition 6.3.2. Let $\mu$ be a $f$-conformal probability measure. We say that $\mu$ is extremal if for all $\mu_{1}, \mu_{2}, t \in(0,1)$ such that $\mu=t \mu_{1}+(1-t) \mu_{2}$, it follows that $\mu_{1}=\mu_{2}=\mu$.

Lemma 6.3.3. Let $\mu$ be a finite measure on the topological space $X$ with $\mu=\mu^{a}+\mu^{c}, \mu^{a}$ purely atomic and $\mu^{c}$ non-atomic. Then $\mu$ is $f$-conformal if and only if $\mu^{a}, \mu^{c}$ are $f$-conformal.

Proof. Since $\mu=\mu^{a}+\mu^{c}$, both $\mu^{a}, \mu^{c}$ are finite measures.

Assume $\mu$ is $f$-conformal. Let $X^{a}$ be the Borel set such that $\mu^{a}\left(X^{a}\right)=\mu^{a}(X)$ and $\mu(x)>0$ for every $x \in X^{a}$. Since $\mu^{a}$ is finite, then $X^{a}$ is countable and $\mu^{c}\left(X^{a}\right)=0$.

Let $A$ be a special set. Since $X^{a}$ is countable, $T\left(A \cap X^{a}\right)$ is also countable, then $T\left(A \cap X^{a}\right)$ is measurable. Note that $T(A)=T\left(A \cap X^{a}\right) \cup T\left(A \backslash X^{a}\right)$ and $T\left(A \cap X^{a}\right) \cap T\left(A \backslash X^{a}\right)=\varnothing$. Hence $T\left(A \backslash X^{a}\right)$ is measurable.

$\mu^{c}\left(T\left(A \cap X^{a}\right)\right)=0$ since $T\left(A \cap X^{a}\right)$ is countable. Now we show $\mu^{a}\left(T\left(A \backslash X^{a}\right)\right)=0$. Let $y \in T\left(A \backslash X^{a}\right)$. There exists $a \in A \backslash X^{a}$ such that $y=T(a)$. Using that $\mu$ is $f$-conformal 
and $a \notin X^{a}$, we have

$$
\mu^{a}(T(a))=\mu(T(a))=f(a) \mu(a)=f(a) \mu^{a}(a)=0 .
$$

Since $y$ is arbitrary and $\mu^{a}$ is atomic, we have $\mu^{a}\left(T\left(A \backslash X^{a}\right)\right)=0$. Then

$$
\begin{aligned}
\mu^{a}(T(A)) & =\mu^{a}\left(T\left(A \cap X^{a}\right)\right)+\mu^{a}\left(T\left(A \backslash X^{a}\right)\right) \\
& =\mu^{a}\left(T\left(A \cap X^{a}\right)\right) \\
& =\mu^{a}\left(T\left(A \cap X^{a}\right)\right)+\mu^{c}\left(T\left(A \cap X^{a}\right)\right) \\
& =\mu\left(T\left(A \cap X^{a}\right)\right) \\
& =\int_{A \cap X^{a}} f(x) d \mu(x) \\
& =\int_{A} f(x) d \mu^{a}(x) .
\end{aligned}
$$

Since $\mu=\mu^{a}$ on $X^{a}$ and $\mu^{a}=0$ outside $X^{a}$, then $\mu^{a}$ is $f$-conformal. The proof for $\mu^{c}$ is analogous.

Conversely, assume $\mu^{a}, \mu^{c}$ are $f$-conformal. Let $A$ be a Borel set such that $T: A \rightarrow T(A)$ is invertible. Then

$$
\begin{aligned}
\mu(T(A)) & =\mu^{a}(T(A))+\mu^{c}(T(A)) \\
& =\int_{A} f(x) d \mu^{a}(x)+\int_{A} f(x) d \mu^{c}(x) \\
& =\int_{A} f(x) d \mu(x) .
\end{aligned}
$$

Then $\mu$ is $f$-conformal.

Remark 6.3.4. Fix a continuous function $F: X \rightarrow \mathbb{R}$ and assume $\beta \neq 0$. It follows from Lemma 6.3 .3 that every extremal $e^{\beta F}$-conformal measure is either purely atomic or non-atomic. In fact, let $\mu$ be an extremal $e^{\beta F}$-conformal probability measure and assume is neither purely atomic nor non-atomic. By Lemma 6.3.3, $\mu=\mu^{a}+\mu^{c}, \mu^{a}$ is purely atomic 
and $\mu^{c}$ is non-atomic.

Let $t=\mu^{a}(X)$. Then $t>0$ (otherwise $\mu=\mu^{c}$ ) and $t<1$ (otherwise $\mu=\mu^{a}$ ). Define $\mu_{1}=t^{-1} \mu^{a}, \mu_{2}=(1-t)^{-1} \mu^{c}$. Note that $\mu_{1}, \mu_{2}$ are probability measures. In fact,

$$
\mu_{1}(X)=t^{-1} \mu^{a}(X)=t^{-1} t=1
$$

and

$$
\begin{aligned}
\mu_{2}(X) & =(1-t)^{-1} \mu^{c}(X) \\
& =(1-t)^{-1}\left(\mu(X)-\mu^{a}(X)\right) \quad, \text { since } \mu=\mu^{a}+\mu^{c}, \\
& =(1-t)^{-1}(1-t)=1 .
\end{aligned}
$$

Then $\mu_{1}, \mu_{2}$ are probability measures. Now let $A$ be a special set. Then

$$
\mu_{1}(T(A))=t^{-1} \mu^{a}(T(A))=t^{-1} \int_{A} e^{\beta F(x)} d \mu^{a}(x)=\int_{A} e^{\beta F(x)} d \mu_{1}(x)
$$

and

$$
\mu_{2}(T(A))=(1-t)^{-1} \mu^{c}(T(A))=(1-t)^{-1} \int_{A} e^{\beta F(x)} d \mu^{c}(x)=\int_{A} e^{\beta F(x)} d \mu_{2}(x) .
$$

Therefore $\mu_{1}, \mu_{2}$ are $e^{\beta F}$-conformal.

In this section we want to find all extremal $e^{\beta F}$-conformal purely atomic probability measures on $X$. Given $\mu e^{\beta F}$-conformal, then $\mu(\sigma(x))=e^{\beta F(x)} \mu(x)$.

Lemma 6.3.5. Let $\beta \in \mathbb{R}$ and $\mu$ an $e^{\beta F}$-conformal measure on $X, n$ a positive natural number. Given $y \in X$, we have

$$
\mu\left(\sigma^{n}(y)\right)=\exp \left(\beta \sum_{k=0}^{n-1} F\left(\sigma^{k}(y)\right)\right) \mu(y)
$$


Proof. We prove this by induction. Assume 6.2 holds for $n$. Then

$$
\begin{aligned}
\mu\left(\sigma^{n+1}(y)\right) & =\mu\left(\sigma\left(\sigma^{n}(y)\right)\right)=e^{\beta F\left(\sigma^{n}(y)\right)} \mu\left(\sigma^{n}(y)\right), \text { since } \mu \text { is } e^{\beta F} \text {-conformal, } \\
& =e^{\beta F\left(\sigma^{n}(y)\right)} \exp \left(\beta \sum_{k=0}^{n-1} F\left(\sigma^{k}(y)\right)\right) \mu(y), \text { by hypothesis, } \\
& =\exp \left(\beta \sum_{k=0}^{n} F\left(\sigma^{k}(y)\right)\right) \mu(y) \\
& =\exp \left(\beta \sum_{k=0}^{(n+1)-1} F\left(\sigma^{k}(y)\right)\right) \mu(y) .
\end{aligned}
$$

Then (6.2) holds for $n+1$. Let $n=1$. Using the fact that $\mu$ is $e^{\beta F}$-conformal, we have

$$
\mu\left(\sigma^{n}(y)\right)=\mu(\sigma(y))=e^{\beta F(y)} \mu(y)=\exp \left(\beta \sum_{k=0}^{n-1} F\left(\sigma^{k}(y)\right)\right) \mu(y) .
$$

Proposition 6.3.6. Given $\beta \in \mathbb{R}$, let $\mu$ be an $e^{\beta F}$-conformal measure on $X, x \in X$. Then, for every $y \in \mathcal{O}(x)$,

$$
\mu(y)=\exp \left(-\beta\left(\sum_{j=0}^{m-1} F\left(\sigma^{j}(y)\right)-\sum_{j=0}^{n-1} F\left(\sigma^{j}(x)\right)\right)\right) \mu(x),
$$

where $\sigma^{n}(x)=\sigma^{m}(y)$.

Proof. Let $y \in \mathcal{O}(x)$. There exist $m, n>0$ such that $\sigma^{n}(x)=\sigma^{m}(y)$. Then

$$
\mu\left(\sigma^{n}(x)\right)=\exp \left(\beta \sum_{j=0}^{n-1} F\left(\sigma^{j}(x)\right)\right) \mu(x) \quad \text { and } \quad \mu\left(\sigma^{m}(y)\right)=\exp \left(\beta \sum_{j=0}^{m-1} F\left(\sigma^{j}(y)\right)\right) \mu(y) .
$$

Since $\sigma^{n}(x)=\sigma^{m}(y)$, it follows that

$$
\exp \left(\beta \sum_{j=0}^{m-1} F\left(\sigma^{j}(y)\right)\right) \mu(y)=\exp \left(\beta \sum_{j=0}^{n-1} F\left(\sigma^{j}(x)\right)\right) \mu(x)
$$




$$
\begin{aligned}
& \mu(y)=\exp \left(\beta \sum_{j=0}^{n-1} F\left(\sigma^{j}(x)\right)\right) \mu(x) \exp \left(-\beta \sum_{j=0}^{m-1} F\left(\sigma^{j}(y)\right)\right) \\
& \mu(y)=\exp \left(-\beta\left(\sum_{j=0}^{m-1} F\left(\sigma^{j}(y)\right)-\sum_{j=0}^{n-1} F\left(\sigma^{j}(x)\right)\right)\right) \mu(x) .
\end{aligned}
$$

It follows from Proposition 6.3.6 that if two $e^{\beta F}$-conformal measures $\mu_{1}, \mu_{2}$ are equal on a point $x \in X$, then $\mu_{1}, \mu_{2}$ are equal on $\mathcal{O}(x)$. Moreover, if $\mu$ is an $e^{\beta F}$-conformal measure such that $\mu(x)>0$, then $\mu(y)>0$ for every $y \in \mathcal{O}(x)$.

Corollary 6.3.7. Let $x \in X$ and $\beta \in \mathbb{R}$. There exists at most one $e^{\beta F}$-conformal probability measure that vanishes outside $\mathcal{O}(x)$. In particular, if $\mu$ is an $e^{\beta F}$-conformal probability measure that vanishes outside $\mathcal{O}(x)$, then $\mu$ is extremal.

Proof. Let $\mu_{1}, \mu_{2}$ be a $e^{\beta F}$-conformal probability measures vanishing outside $\mathcal{O}(x)$. It follows from Proposition 6.3.6 that $\mu_{1}(x), \mu_{2}(x)>0$. In fact, let $i=1,2$. Since $\mathcal{O}(x)$ is countable and $\mu_{i}$ is a probability measure whose support lies in $\mathcal{O}(x)$, there exists $y \in \mathcal{O}(x)$ such that $\mu_{i}(y)>0$. Then $\mu_{i}(x)>0$ by Proposition 6.3.6.

Suppose that $\mu_{1}(x)<\mu_{2}(x)$. Then $\mu_{1}(y)<\mu_{2}(y)$ for every $y \in \mathcal{O}(x)$ by Proposition 6.3.6. Therefore

$$
1=\mu_{1}(X)=\mu_{1}(\mathcal{O}(x))=\mu_{2}(\mathcal{O}(x))=\mu_{2}(X)=1
$$

which is a contradiction. is not a probability measure. The proof is analogous for $\mu_{1}(x)>$ $\mu_{2}(x)$. Then $\mu_{1}(x)=\mu_{2}(x)$. Then $\mu_{1}$ and $\mu_{2}$ are equal on $\mathcal{O}(x)$ by Proposition 6.3.6. Therefore $\mu_{1}=\mu_{2}$.

Now we show $\mu$ is extremal. Let $\mu_{1}, \mu_{2}$ be two $e^{\beta F}$-conformal probability measures such that $\mu=t \mu_{1}+(1-t) \mu_{2}, 0<t<1$. Let $A=X \backslash \mathcal{O}(x)$. $A$ is measurable. Since $\mu(A)=0$ and $\mu_{1}(A), \mu_{2}(A) \geq 0$, we have $\mu_{1}(A)=\mu_{2}(A)=0$. By previous arguments, $\mu=\mu_{1}=\mu_{2}$. Therefore $\mu$ is extremal. 
Corollary 6.3.8. Assume $\beta \in \mathbb{R} \backslash\{0\}$. Let $\mu$ be an $e^{\beta F}$-conformal measure. Let $x \in X$ periodic with minimum period $p$ such that $\mu(x)>0$. It follows that

$$
\sum_{j=0}^{p-1} F\left(\sigma^{j}(x)\right)=0
$$

Proof. $\sigma^{p}(x)=x$. Then,

$$
\mu(x)=\mu\left(\sigma^{p}(x)\right)=\exp \left(\beta \sum_{k=0}^{p-1} F\left(\sigma^{k}(x)\right)\right) \mu(x)
$$

$\mu(x)>0$, hence

$$
1=\exp \left(\beta \sum_{k=0}^{p-1} F\left(\sigma^{k}(x)\right)\right)
$$

Note that $\beta \neq 0$. Therefore $\sum_{k=0}^{p-1} F\left(\sigma^{k}(x)\right)=0$.

Lemma 6.3.9. Let $\mu$ be a purely atomic extremal $e^{\beta F}$-conformal probability measure, $\beta \in \mathbb{R}$. Then there exists $x \in X$ such that for $y \in X, \mu(y)>0$ if, and only if, $y \in \mathcal{O}(x)$.

Proof. Let $x \in X$ such that $\mu(x)>0$.

Suppose there exists $y \in X \backslash \mathcal{O}(x)$ such that $\mu(y)>0$. Since $\mu$ is a probability measure, $\mu(x)>0$ and $x \notin \mathcal{O}(y)$, we have $0<\mu(\mathcal{O}(y))<1$. Let $t=\mu(\mathcal{O}(y))$. Define $\mu_{1}$ by

$$
\mu_{1}(z)= \begin{cases}t^{-1} \mu(z) & \text { if } z \in \mathcal{O}(y) \\ 0 & \text { otherwise }\end{cases}
$$

Then $\mu_{1}$ is a probability measure. Note that $\sigma(z) \in \mathcal{O}(y)$ if, and only if, $z \in \mathcal{O}(y)$. In fact, $\mathcal{O}(\sigma(z))=\mathcal{O}(z)$ from Lemma 6.2.2. Then

$$
\mu_{1}(\sigma(z))=t^{-1} \mu(\sigma(z))=e^{\beta F(z)} t^{-1} \mu(z)=e^{\beta F(z)} \mu_{1}(z)
$$


Therefore $\mu_{1}$ is $e^{\beta F}$-conformal. Define $\mu_{2}$ by

$$
\mu_{2}(z)= \begin{cases}(1-t)^{-1} \mu(z) & \text { if } z \notin \mathcal{O}(y) \\ 0 & \text { if } z \in \mathcal{O}(y)\end{cases}
$$

$\mu_{2}$ is an $e^{\beta F}$-conformal probability measure as well. In fact,

$$
\begin{aligned}
\mu_{2}(X) & =\mu_{2}(X \backslash \mathcal{O}(y)) \\
& =(1-t)^{-1} \mu(X \backslash \mathcal{O}(y)) \\
& =(1-t)^{-1}[\mu(X)-\mu(\mathcal{O}(y))] \\
& =(1-t)^{-1}[1-t] \quad, \text { since } t=\mu(\mathcal{O}(x)), \\
& =1 .
\end{aligned}
$$

Then $\mu_{2}$ is a probability measure. Given $z \notin \mathcal{O}(y), \sigma(z) \notin \mathcal{O}(y)$. In fact, suppose $\sigma(z) \in$ $\mathcal{O}(y)$. Then $\mathcal{O}(\sigma(z))=\mathcal{O}(y)$ by Lemma 6.2.2, but $\mathcal{O}(z)=\mathcal{O}(\sigma(z))$. Then $\mathcal{O}(\sigma(z))=\mathcal{O}(y)$. Therefore $z \in \mathcal{O}(y)$. Contradiction. Then,

$$
\begin{aligned}
\mu_{2}(\sigma(z)) & =(1-t)^{-1} \mu(\sigma(z)) \\
& =e^{\beta F(z)}(1-t)^{-1} \mu(z) \quad, \text { since } \mu \text { is } e^{\beta F} \text {-conformal } \\
& =e^{\beta F(z)} \mu_{2}(z) .
\end{aligned}
$$

Therefore $\mu_{2}$ is $e^{\beta F}$-conformal.

Moreover, $\mu=t \mu_{1}+(1-t) \mu_{2}$. Therefore $\mu$ is not extremal. Contradiction. Then $\mu(y)=0$ if $y \notin \mathcal{O}(x)$.

Let $y \in \mathcal{O}(x)$. It follows from Proposition 6.3.6 that $\mu(y)>0$.

For $\beta \neq 0$, each extremal atomic $e^{\beta F}$-conformal probability measure corresponds to an orbit $\mathcal{O}(x)$. However, an orbit $\mathcal{O}(x)$ does not necessarily have a correspondent extremal atomic $e^{\beta F}$-conformal probability measure. 
Proposition 6.3.10. Let $\beta \in \mathbb{R} \backslash\{0\}$. Let $x \in X$ be a periodic point with minimum period $p$. There exists an extremal atomic $e^{\beta F}$-conformal probability measure with support $\mathcal{O}(x)$ if, and only if,

$$
\begin{gathered}
\sum_{j=0}^{p-1} F\left(\sigma^{j}(x)\right)=0, \\
M=\sum_{n=1}^{\infty} \sum_{y \in Y_{n}} \exp \left(-\beta \sum_{j=0}^{n-1} F\left(\sigma^{j}(y)\right)\right)<\infty .
\end{gathered}
$$

where $\quad Y_{n}=\sigma^{-n}(x) \backslash \bigcup_{j=0}^{n-1} \sigma^{-j}(x)$ for $n \geq 1$, and $Y_{0}=\{x\}$.

In this case, the measure is denoted by

$$
m_{x}=(1+M)^{-1}\left[\delta_{x}+\sum_{n=1}^{\infty} \sum_{y \in Y_{n}} \exp \left(-\beta \sum_{j=0}^{n-1} F\left(\sigma^{j}(y)\right)\right) \delta_{y}\right] .
$$

Proof. Let $\mu$ be an extremal atomic $e^{\beta F}$-conformal probability measure with support $\mathcal{O}(x)$. It follows from Corollary 6.3 .8 that 6.3$)$ holds.

Let $y \in \mathcal{O}(x)$, by Lemma 6.2 .8 there exists a minimum natural number $n$ such that $\sigma^{n}(y)=x$. Hence $y \in Y_{n}$. Therefore

$$
\mathcal{O}(x)=\bigcup_{n=0}^{\infty} Y_{n} \quad \text { and } \quad \mu(\mathcal{O}(x))=\sum_{n=0}^{\infty} \mu\left(Y_{n}\right) .
$$

Let $y \in Y_{n}$. From Proposition 6.3.6,

$$
\mu(y)=\exp \left(-\beta \sum_{j=0}^{n-1} F\left(\sigma^{j}(y)\right)\right) \mu(x) .
$$


$\mathcal{O}(x)$ is countable by Lemma 6.2.4. Thus,

$$
\mu\left(Y_{n}\right)=\sum_{y \in Y_{n}} \mu(y)=\sum_{y \in Y_{n}} \exp \left(-\beta \sum_{j=0}^{n-1} F\left(\sigma^{j}(y)\right)\right) \mu(x)
$$

Hence,

$$
\begin{aligned}
1 & =\mu(\mathcal{O}(x))=\sum_{n=0}^{\infty} \mu\left(Y_{n}\right) \\
& =\mu(x)+\sum_{n=1}^{\infty} \sum_{y \in Y_{n}} \exp \left(-\beta \sum_{j=0}^{n-1} F\left(\sigma^{j}(y)\right)\right) \mu(x) \\
& =\mu(x)\left[1+\sum_{n=1}^{\infty} \sum_{y \in Y_{n}} \exp \left(-\beta \sum_{j=0}^{n-1} F\left(\sigma^{j}(y)\right)\right)\right] \\
& =\mu(x)(1+M) .
\end{aligned}
$$

Then $M<\infty$ and $\mu(x)=(1+M)^{-1}$. Given $y \in Y_{n}, n \geq 1$,

$$
\mu(y)=(1+M)^{-1} \exp \left(-\beta \sum_{j=0}^{n-1} F\left(\sigma^{j}(y)\right)\right)
$$

Therefore 6.5 holds.

Conversely, assume 6.3), 6.4 hold. Given $n>1$,

$$
m_{x}\left(Y_{n}\right)=(1+M)^{-1} \sum_{y \in Y_{n}} \exp \left(-\beta \sum_{j=0}^{n-1} F\left(\sigma^{j}(y)\right)\right) .
$$

Hence,

$$
\begin{aligned}
m_{x}(\mathcal{O}(x)) & =m_{x}(x)+\sum_{n=1}^{\infty} m_{x}\left(Y_{n}\right) \\
& =(1+M)^{-1}\left[1+\sum_{n=1}^{\infty} \sum_{y \in Y_{n}} \exp \left(-\beta \sum_{j=0}^{n-1} F\left(\sigma^{j}(y)\right)\right)\right]
\end{aligned}
$$




$$
=(1+M)^{-1}(1+M)=1 .
$$

Then $m_{x}$ is a probability measure. Now we prove $m_{x}$ is $e^{\beta F}$-conformal.

Let $y \in Y_{n}, n \geq 2$. Then $\sigma(y) \in Y_{n-1}$. In fact, $\sigma^{n-1}(\sigma(y))=\sigma^{n}(y)=x$. Let $0 \leq l<n-1$, then $\sigma^{l}(\sigma(y))=\sigma^{l+1}(y) \neq x$, since $1 \leq l<n$. Let $y^{\prime}=\sigma(y)$, then

$$
\begin{aligned}
m_{x}(\sigma(y)) & =(1+M)^{-1} \exp \left(-\beta \sum_{j=0}^{n-2} F\left(\sigma\left(y^{\prime}\right)\right)\right) \\
& =(1+M)^{-1} \exp \left(-\beta \sum_{j=1}^{n-1} F(\sigma(y))\right) \\
& =e^{\beta F(y)}(1+M)^{-1} \exp \left(-\beta \sum_{j=0}^{n-1} F(\sigma(y))\right) \\
& =e^{\beta F(y)} m_{x}(y) .
\end{aligned}
$$

Let $y \in Y_{n}, n=1$. Then $\sigma(y)=x$. Hence,

$$
\begin{aligned}
m_{x}(\sigma(y)) & =m_{x}(x)=(1+M)^{-1} \\
& =e^{\beta F(y)}(1+M)^{-1} \exp (-\beta F(y)) \\
& =e^{\beta F(y)}(1+M)^{-1} \exp \left(-\beta \sum_{j=0}^{n-1} F\left(\sigma^{j}(y)\right)\right) \\
& =e^{\beta F(y)} m_{x}(y) .
\end{aligned}
$$

Note that $\sigma(x) \in Y_{p-1}$. In fact, $\sigma^{p-1}(\sigma(x))=\sigma^{p}(x)=x$. Given $0 \leq l<p-1, \sigma^{l}(\sigma(x))=$ $\sigma^{l+1}(x) \neq x$ since $1 \leq l+1<p$. Then, if $x^{\prime}=\sigma(x)$, we have

$$
\begin{aligned}
m_{x}(\sigma(x)) & =(1+M)^{-1} \exp \left(-\beta \sum_{j=0}^{p-2} F\left(\sigma^{j}\left(x^{\prime}\right)\right)\right) \\
& =(1+M)^{-1} \exp \left(-\beta \sum_{j=1}^{p-1} F\left(\sigma^{j}(x)\right)\right)
\end{aligned}
$$




$$
\begin{aligned}
& =e^{\beta F(x)}(1+M)^{-1} \exp \left(-\beta \sum_{j=0}^{p-1} F\left(\sigma^{j}(x)\right)\right) \\
& =e^{\beta F(x)}(1+M)^{-1} \quad \text { from } 6.3 \\
& =e^{\beta F(x)} m_{x}(x) .
\end{aligned}
$$

Therefore $m_{x}$ is $e^{\beta F}$-conformal. $m_{x}$ has support $\mathcal{O}(x)$ by definition. From Corollary 6.3.7. $m_{x}$ is extremal.

Definition 6.3.11. Given a continuous function $F: X \rightarrow \mathbb{R}$ we define $c_{F}: \mathcal{G} \rightarrow \mathbb{R}$ by

$$
c_{F}(x, k, y)=\sum_{j=0}^{n-1} F\left(\sigma^{j}(x)\right)-\sum_{j=0}^{m-1} F\left(\sigma^{j}(y)\right),
$$

for $n, m \in \mathbb{N}$ such that $k=n-m$ and $\sigma^{n}(x)=\sigma^{m}(y)$.

Lemma 6.3.12. $c_{F}$ is well-defined.

Proof. Let $(x, k, y) \in \mathcal{G}$. For $i=1,2$, let $n_{i}, m_{i} \in \mathbb{N}$ such that $\sigma^{n_{i}}(x)=\sigma^{m_{i}}(y), k=n_{i}-m_{i}$. Then $n_{2}-n_{1}=m_{2}-m_{1}$. Assume $n_{2}>n_{1}$ without loss of generality. Then $m_{2}>m_{1}$ and

$$
\begin{aligned}
& \sum_{j=0}^{n_{2}-1} F\left(\sigma^{j}(x)\right)-\sum_{j=0}^{m_{2}-1} F\left(\sigma^{j}(y)\right) \\
= & \left(\sum_{j=0}^{n_{1}-1} F\left(\sigma^{j}(x)\right)+\sum_{j=n_{1}}^{n_{2}-1} F\left(\sigma^{j}(x)\right)\right)-\left(\sum_{j=0}^{m_{1}-1} F\left(\sigma^{j}(y)\right)+\sum_{j=m_{1}}^{m_{2}-1} F\left(\sigma^{j}(y)\right)\right) \\
= & \left(\sum_{j=0}^{n_{1}-1} F\left(\sigma^{j}(x)\right)+\sum_{j=0}^{n_{2}-n_{1}-1} F\left(\sigma^{j+n_{1}}(x)\right)\right)-\left(\sum_{j=0}^{m_{1}-1} F\left(\sigma^{j}(y)\right)+\sum_{j=0}^{m_{2}-m_{1}-1} F\left(\sigma^{j+m_{1}}(y)\right)\right) \\
= & \left(\sum_{j=0}^{n_{1}-1} F\left(\sigma^{j}(x)\right)-\sum_{j=0}^{m_{1}-1} F\left(\sigma^{j}(y)\right)\right)+\left(\sum_{j=0}^{n_{2}-n_{1}-1} F\left(\sigma^{j+n_{1}}(x)\right)-\sum_{j=0}^{m_{2}-m_{1}-1} F\left(\sigma^{j+m_{1}}(y)\right)\right) \\
= & \left(\sum_{j=0}^{n_{1}-1} F\left(\sigma^{j}(x)\right)-\sum_{j=0}^{m_{1}-1} F\left(\sigma^{j}(y)\right)\right)+\left(\sum_{j=0}^{n_{2}-n_{1}-1} F\left(\sigma^{j}\left(\sigma^{n_{1}}(x)\right)\right)-\sum_{j=0}^{n_{1}-1} F\left(\sigma^{j}\left(\sigma^{m_{1}}(y)\right)\right)\right) \\
= & \left(\sum_{j=0}^{n_{1}-1} F\left(\sigma^{j}(x)\right)-\sum_{j=0}^{m_{1}-1} F\left(\sigma^{j}(y)\right)\right)+\left(\sum_{j=0}^{n_{2}-n_{1}-1} F\left(\sigma^{j}\left(\sigma^{m_{1}}(y)\right)\right)-\sum_{j=0} F\left(\sigma^{j}\left(\sigma^{m_{1}}(y)\right)\right)\right)
\end{aligned}
$$




$$
=\left(\sum_{j=0}^{n_{1}-1} F\left(\sigma^{j}(x)\right)-\sum_{j=0}^{m_{1}-1} F\left(\sigma^{j}(y)\right)\right) .
$$

Therefore $c_{F}(x, k, y)$ does not depend on the choice of $n, m$ satisfying $k=n-m$.

Proposition 6.3.13. $c_{F}$ is a continuous $\mathbb{R}$-valued 1 -cocycle on $\mathcal{G}$.

Proof. Let $(x, k, y),(y, l, z) \in \mathcal{G}$. There exist $m, n, p, q \in \mathbb{N}$ such that $k=m-n, l=p-q$,

$$
\sigma^{m}(x)=\sigma^{n}(y) \quad \text { and } \quad \sigma^{p}(y)=\sigma^{q}(z) .
$$

Then $m+p-n-q=k+l$ and $\sigma^{m+p}(x)=\sigma^{n+p}(y)=\sigma^{n+q}(z)$. Hence,

$$
\begin{aligned}
c_{F}(x, k+l, z) & =\sum_{j=0}^{m+p-1} F\left(\sigma^{j}(x)\right)-\sum_{j=0}^{n+q-1} F\left(\sigma^{j}(z)\right) \\
& =\sum_{j=0}^{m-1} F\left(\sigma^{j}(x)\right)+\sum_{j=m}^{m+p-1} F\left(\sigma^{j}(x)\right)-\sum_{j=0}^{q-1} F\left(\sigma^{j}(z)\right)-\sum_{j=q}^{n+q-1} F\left(\sigma^{j}(z)\right) \\
& =\sum_{j=0}^{m-1} F\left(\sigma^{j}(x)\right)+\sum_{j=0}^{p-1} F\left(\sigma^{j+m}(x)\right)-\sum_{j=0}^{q-1} F\left(\sigma^{j}(z)\right)-\sum_{j=0}^{n-1} F\left(\sigma^{j+q}(z)\right) \\
& =\sum_{j=0}^{m-1} F\left(\sigma^{j}(x)\right)+\sum_{j=0}^{p-1} F\left(\sigma^{j+n}(y)\right)-\sum_{j=0}^{q-1} F\left(\sigma^{j}(z)\right)-\sum_{j=0}^{n-1} F\left(\sigma^{j+p}(y)\right) \\
& =\sum_{j=0}^{m-1} F\left(\sigma^{j}(x)\right)+\sum_{j=n}^{p+n-1} F\left(\sigma^{j}(y)\right)-\sum_{j=0}^{q-1} F\left(\sigma^{j}(z)\right)-\sum_{j=p}^{p+n} F\left(\sigma^{j}(y)\right) \\
& =\sum_{j=0}^{m-1} F\left(\sigma^{j}(x)\right)-\sum_{j=0}^{n-1} F\left(\sigma^{j}(y)\right)+\sum_{j=0}^{p+n-1} F\left(\sigma^{j}(y)\right) \\
& -\sum_{j=0}^{q-1} F\left(\sigma^{j}(z)\right)+\sum_{j=0}^{p-1} F\left(\sigma^{j}(y)\right)-\sum_{j=0}^{p+n-1} F\left(\sigma^{j}(y)\right) \\
& =c_{F}(x, k, y)+c_{F}(y, l, z) .
\end{aligned}
$$

Now we prove the continuity of $c_{F}$. Let $\left(x_{i}, k_{i}, y_{i}\right) \rightarrow(x, k, y)$. Then $x_{i} \rightarrow x$ and $y_{i} \rightarrow y$. There exists $m, n \in \mathbb{N}$ with $m-n=k$ and $i_{0}$ such that $\sigma^{m}\left(x_{i}\right)=\sigma^{n}\left(y_{i}\right)$ for every $i \geq i_{0}$. By 
continuity of $\sigma, \sigma^{m}(x)=\sigma^{n}(y)$.

Hence, for every $i \geq i_{0}$,

$$
c_{F}\left(x_{i}, k_{i}, y_{i}\right)=\sum_{j=0}^{m-1} F\left(\sigma^{j}\left(x_{i}\right)\right)-\sum_{j=0}^{n-1} F\left(\sigma^{j}\left(y_{i}\right)\right) .
$$

Since $F$ and $\sigma$ are continuous on $X$, we have $c_{F}\left(x_{i}, k_{i}, y_{i}\right) \rightarrow c_{F}(x, k, y)$.

The following lemma will be used to prove that every continuous $\mathbb{R}$-valued 1-cocycle corresponds to a unique $c_{F}$.

Lemma 6.3.14. Let $(x, n-m, y) \in \mathcal{G}$ such that $\sigma^{n}(x)=\sigma^{m}(y)$. Suppose that $n, m \geq 1$. Then

$$
\begin{aligned}
(x, n-m, y)= & (x, 1, \sigma(x))\left(\sigma(x), 1, \sigma^{2}(x)\right) \cdots\left(\sigma^{n-1}(x), 1, \sigma^{n}(x)\right) \\
& \left(\sigma^{m}(y),-1, \sigma^{m-1}(y)\right)\left(\sigma^{m-1}(y),-1, \sigma^{m-2}(y)\right) \cdots(\sigma(y),-1, y) .
\end{aligned}
$$

Proof. First we show that for every natural number $N \geq 1$,

$$
\left(x, N, \sigma^{N}(x)\right)=(x, 1, \sigma(x)) \cdots\left(\sigma^{N-1}(x), 1, \sigma^{N}(x)\right) .
$$

Clearly (6.7) holds for $N=1$. Suppose that 6.7 is satisfied for an arbitrary $N$. Then

$$
\begin{aligned}
\left(x, N+1, \sigma^{N+1}(x)\right) & =\left(x, N, \sigma^{N}(x)\right)\left(\sigma^{N}(x), 1, \sigma^{N+1}(x)\right) \\
& =(x, 1, \sigma(x)) \cdots\left(\sigma^{N}(x), 1, \sigma^{N+1}(x)\right) .
\end{aligned}
$$

Hence, (6.7) is satisfied for every $N$. In particular, this equality holds for $N=n$. By the same argument,

$$
\left(y, m, \sigma^{m}(y)\right)=(y, 1, \sigma(y)) \cdots\left(\sigma^{m-1}(y), 1, \sigma^{m}(y)\right) .
$$


Then, applying the inverse on both sides, we have

$$
\left(\sigma^{m}(y),-m, y\right)=\left(\sigma^{m}(y),-1, \sigma^{m-1}(y)\right) \cdots(\sigma(y),-1, y)
$$

Since $\sigma^{n}(x)=\sigma^{m}(y)$, we have

$$
\begin{aligned}
(x, n-m, y)= & \left(x, n, \sigma^{n}(x)\right)\left(\sigma^{m}(y),-m, y\right) \\
= & (x, 1, \sigma(x))\left(\sigma(x), 1, \sigma^{2}(x)\right) \cdots\left(\sigma^{n-1}(x), 1, \sigma^{n}(x)\right) \\
& \left(\sigma^{m}(y),-1, \sigma^{m-1}(y)\right)\left(\sigma^{m-1}(y),-1, \sigma^{m-2}(y)\right) \cdots(\sigma(y),-1, y) .
\end{aligned}
$$

Now we will prove that there exists a bijection between continuous $\mathbb{R}$-valued 1-cocycles on $\mathcal{G}$ and the continuous functions from $X$ to $\mathbb{R}$. Recall from Proposition 6.3.13 that every $c_{F}$ is a continuous $\mathbb{R}$-valued 1-cocycle.

Proposition 6.3.15. For every continuous $\mathbb{R}$-valued 1 -cocycle $c$ on $\mathcal{G}$, there exists a unique continuous function $F: X \rightarrow \mathbb{R}$ such that $c=c_{F}$.

Proof. Let $c: \mathcal{G} \rightarrow \mathbb{R}$ be a continuous $\mathbb{R}$-valued 1-cocycle. Define the function $F: X \rightarrow \mathcal{G}$ by $F(x)=c(x, 1, \sigma(x))$.

The function $F$ is continuous. In fact, let $\left\{x_{n}\right\}_{n \in \mathbb{N}}$ be a sequence in $X$ converging to $x \in X$. By definition of $\mathcal{G},(x, 1, \sigma(x)) \in \mathcal{G}$ and each $\left(x_{n}, 1, \sigma\left(x_{n}\right)\right) \in \mathcal{G}$. Since $\sigma$ is continuous, we have $\sigma\left(x_{n}\right) \rightarrow \sigma(x)$. It follows from Corollary 6.1.8 that $\left(x_{n}, 1, \sigma\left(x_{n}\right)\right) \rightarrow(x, 1, \sigma(x))$. Since $c$ is continuous, we have $F\left(x_{n}\right) \rightarrow F(x)$. Therefore $F$ is continuous.

Let $(x, n-m, y) \in \mathcal{G}$ such that $\sigma^{n}(x)=\sigma^{m}(y)$. Assume that $n, m \geq 1$ without loss of generality. Then, by Lemma 6.3.14.

$$
\begin{aligned}
(x, n-m, y) & =(x, 1, \sigma(x))\left(\sigma(x), 1, \sigma^{2}(x)\right) \cdots\left(\sigma^{n-1}(x), 1, \sigma^{n}(x)\right) \\
& =\left(\sigma^{m}(y),-1, \sigma^{m-1}(y)\right)\left(\sigma^{m-1}(y),-1, \sigma^{m-2}(y)\right) \cdots(\sigma(y),-1, y) .
\end{aligned}
$$


Then

$$
\begin{aligned}
c(x, n-m, y)= & c(x, 1, \sigma(x))+\cdots+c\left(\sigma^{n-1}(x), 1, \sigma^{n}(x)\right) \\
& +c\left(\sigma^{m}(y),-1, \sigma^{m-1}(y)\right)+\cdots+c(\sigma(y),-1, y) . \\
= & \sum_{i=0}^{n-1} c\left(\sigma^{i}(x), 1, \sigma^{i+1}(x)\right)+\sum_{i=0}^{m-1} c\left(\sigma^{i+1}(y),-1, \sigma^{i}(y)\right) \\
= & \sum_{i=0}^{n-1} c\left(\sigma^{i}(x), 1, \sigma^{i+1}(x)\right)-\sum_{i=0}^{m-1} c\left(\sigma^{i}(y), 1, \sigma^{i+1}(y)\right) \\
= & \sum_{i=0}^{n-1} F\left(\sigma^{i}(x)\right)-\sum_{i=0}^{m-1} F\left(\sigma^{i}(y)\right) \\
= & c_{F}(x, n-m, y) .
\end{aligned}
$$

Finally we prove that $c_{F}$ is unique. Suppose that there exists a continuous function $H: X \rightarrow \mathbb{R}$ such that $c_{H}=c_{F}$. Then, for every $x \in X$,

$$
H(x)=c_{H}(x, 1, \sigma(x))=c_{F}(x, 1, \sigma(x))=F(x)
$$

Therefore $H=F$.

The following lemma shows a equality for $c_{F}$ on $\mathcal{G}_{y}^{y}$ where $\mathcal{O}(y)$ is periodic.

Lemma 6.3.16. Let $x \in X$ be a periodic point with minimum period $p$. Then

$$
c_{F}(y, k p, y)=k \sum_{j=0}^{p-1} F\left(\sigma^{j}(x)\right)
$$

for every $y \in \mathcal{O}(x), k \in \mathbb{Z}$.

Proof. Since $\sigma^{p}(x)=x$, we have

$$
c_{F}(x, p, x)=\sum_{j=0}^{p-1} F\left(\sigma^{j}(x)\right) .
$$


Let $y \in \mathcal{O}(x)$. Then, from Lemma 6.2.8, there exists $n$ such that $\sigma^{n}(y)=x$. Thus $(y, n, x) \in \mathcal{G}$. Then

$$
\begin{aligned}
c_{F}(y, k p, y) & =k c_{F}(y, p, y) \\
& =k c_{F}((y, n, x)(x, p, x)(x,-n, y)) \\
& =k\left[c_{F}(y, n, x)+c_{F}(x, p, x)+c_{F}(x,-n, y)\right] \\
& =k\left[c_{F}(y, n, x)+c_{F}(x, p, x)-c_{F}(x,-n, y)\right] \\
& =k c_{F}(x, p, x) \\
& =k \sum_{j=0}^{p-1} F\left(\sigma^{j}(x)\right) .
\end{aligned}
$$

Proposition 6.3.17. Let $\beta \neq 0$. Let $z \in X$ be aperiodic. There exists an extremal atomic $e^{\beta F}$-conformal probability measure with support $\mathcal{O}(z)$ if, and only if,

$$
M=\sum_{y \in \mathcal{O}(z)} e^{-\beta \mathcal{F}(y)}<\infty
$$

where

$$
\mathcal{F}(y)=\sum_{j=0}^{m-1} F\left(\sigma^{j}(y)\right)-\sum_{j=0}^{n-1} F\left(\sigma^{j}(z)\right)
$$

with $\sigma^{m}(y)=\sigma^{n}(z)$. In this case the measure is defined by

$$
m_{z}=M^{-1} \sum_{y \in \mathcal{O}(z)} e^{-\beta \mathcal{F}(y)} \delta_{y}
$$

In particular, if $z$ satisfies condition $(6.8)$, we say $z$ is $\beta$-summable.

Proof. First we prove $\mathcal{F}$ is well-defined. Let $y \in \mathcal{O}(z)$. There exists a unique $k \in \mathbb{Z}$ such that $(z, k, y) \in \mathcal{G}$. In fact, suppose there are $k_{1}, k_{2} \in \mathbb{Z}$ satisfying $\left(z, k_{1}, y\right),\left(z, k_{2}, y\right) \in \mathcal{G}$. 
Then $\left(z, k_{2}-k_{1}, z\right) \in \mathcal{G} . k_{2}-k_{1}=0$ from Lemma 6.2.10.

Let $m, n \in \mathbb{N}$ such that $\sigma^{m}(y)=\sigma^{n}(z)$. Then $(z, n-m, y) \in \mathcal{G}$. Hence $k=n-m$ and

$$
\sum_{j=0}^{m-1} F\left(\sigma^{j}(y)\right)-\sum_{j=0}^{n-1} F\left(\sigma^{j}(z)\right)=-c_{F}(z, k, y)
$$

Therefore $\mathcal{F}(y)$ does not depend on the choice of $m, n$.

Let $\mu$ be an extremal atomic $e^{\beta F}$-conformal probability measure with support $\mathcal{O}(z)$. Given $y \in \mathcal{O}(z)$ there exist $m, n \in \mathbb{N}$ such that $\sigma^{m}(y)=\sigma^{n}(z)$. Then by Proposition 6.3.6.

$$
\mu(y)=\exp \left(-\beta\left(\sum_{j=0}^{m-1} F\left(\sigma^{j}(y)\right)-\sum_{j=0}^{n-1} F\left(\sigma^{j}(z)\right)\right)\right) \mu(z)=e^{-\beta \mathcal{F}(y)} \mu(z)
$$

Since $\mu$ is a probability measure, it follows that

$$
1=\mu(\mathcal{O}(z))=\sum_{y \in \mathcal{O}(z)} \mu(y)=\sum_{y \in \mathcal{O}(z)} e^{-\beta \mathcal{F}(y)} \mu(z)
$$

Hence,

$$
\mu(z)=\left(\sum_{y \in \mathcal{O}(z)} e^{-\beta \mathcal{F}(y)}\right)^{-1}=M^{-1}
$$

Therefore $M<\infty$ and $\mu(y)=M^{-1} e^{-\beta \mathcal{F}(y)}$ for $y \in \mathcal{O}(z)$.

Conversely, assume 6.8 holds. We prove that $m_{z}$ is an extremal $e^{\beta F}$-conformal probability measure.

$$
m_{z}(\mathcal{O}(z))=M^{-1} \sum_{y \in \mathcal{O}(z)} e^{-\beta \mathcal{F}(y)}=M^{-1} M=1
$$

then $m_{z}$ is a probability measure. Now we show $m_{z}$ is $e^{\beta F}$-conformal. Let $y \in \mathcal{O}(z), n, m \in \mathbb{N}$ 
such that $\sigma^{m}(y)=\sigma^{n}(z), m \geq 2$. Then $\sigma^{m-1}(\sigma(y))=\sigma^{n}(z)$ and

$$
\begin{aligned}
\mathcal{F}(\sigma(y)) & =\sum_{j=0}^{m-2} F\left(\sigma^{j}(\sigma(y))\right)-\sum_{j=0}^{n-1} F\left(\sigma^{j}(z)\right)=\sum_{j=1}^{m-1} F\left(\sigma^{j}(y)\right)-\sum_{j=0}^{n-1} F\left(\sigma^{j}(z)\right) \\
& =-F(y)+\sum_{j=0}^{m-1} F\left(\sigma^{j}(y)\right)-\sum_{j=0}^{n-1} F\left(\sigma^{j}(z)\right) \\
& =-F(y)+\mathcal{F}(y) .
\end{aligned}
$$

Then,

$$
m_{z}(\sigma(y))=M^{-1} e^{-\beta \mathcal{F}(\sigma(y))}=M^{-1} e^{-\beta[-F(y)+\mathcal{F}(y)]}=M^{-1} e^{\beta F(y)} e^{-\beta \mathcal{F}(y)}=e^{\beta F(y)} m_{z}(y) .
$$

Therefore $m_{z}$ is $e^{\beta F}$-conformal. By definition of $m_{z}$, its support is $\mathcal{O}(z)$. From Corollary 6.3.7, $m_{z}$ is extremal.

Lemma 6.3.18. Let $X$ be a locally compact second countable Hausdorff topological space, $\mu$ a Borel measure which is finite on compact subsets of $X$. Given a local homeomorphism $\sigma: X \rightarrow X$, and a non-negative function $f$ on $X, \mu$ is $f$-conformal if, and only if,

$$
\mu(\sigma(A))=\int_{A} f(x) d \mu(x),
$$

for every open set $A$ such that $\left.\sigma\right|_{A}$ is injective.

Proof. The measure $\mu$ is Radon by Proposition 2.4.10. Assume $\mu$ is $f$-conformal, then (6.11) holds by definition.

Conversely, suppose (6.11) holds. Let $A$ be a measurable subset such that $\sigma$ is injective on $A$. First we assume there exists an open set $U$ such that $A \subset U$ and $\sigma$ is injective on $U$.

Given an open set $W$ including $\sigma(A)$, there exists an open set $W^{\prime}=W \cap \sigma(U)$ such that $\sigma(A) \subset W^{\prime} \subset \sigma(U)$ and $\mu\left(W^{\prime}\right) \leq \mu(W)$. Since $\mu$ is a Radon measure and $W$ is arbitrary, we 
have

$$
\mu(\sigma(A))=\inf _{\substack{\sigma(A) \subset W \\ W \text { open }}} \mu(W)=\inf _{\substack{\sigma(A) \subset W^{\prime} \subset \sigma(U) \\ W^{\prime} \text { open }}} \mu\left(W^{\prime}\right)
$$

The function $\sigma$ is injective on $U$, then for every open set $W$ satisfying $\sigma(A) \subset W \subset \sigma(U)$, there is a unique open set $V$ such that $A \subset V \subset U$ and $W=\sigma(V)$. Clearly $\sigma$ is injective on each $V$. Then,

$$
\begin{aligned}
\mu(\sigma(A)) & =\inf _{\substack{A \subset V \subset U \\
V \text { open }}} \mu(\sigma(V)) \\
& =\inf _{\substack{A \subset V \subset U \\
V \text { open }}} \int_{V} f(x) d \mu(x), \text { by hypothesis, } \\
& =\int_{A} f(x) d \mu(x), \text { by Lemma 2.4.13. }
\end{aligned}
$$

Now let $A$ be an arbitrary measurable set $A$ such that $\left.\sigma\right|_{A}$ is injective. Since $\sigma$ is a local homeomorphism and $X$ is second countable, there exists a countable open cover $\left\{U_{n}\right\}_{n \in \mathbb{N}}$ of $A$ such that $\sigma$ is injective on each $U_{n}$. Define $A_{1}=A \cap U_{1}$ and, for every $n$,

$$
A_{n+1}=A \cap U_{n+1} \backslash \bigcup_{j=1}^{n} A_{j}
$$

Then $A=\cup_{n=1}^{\infty} A_{n}$ and the family $\left\{A_{n}\right\}_{n \in \mathbb{N}}$ is disjoint. Moreover, $A_{n} \subset U_{n}$ for every $n$. Then,

$$
\mu\left(\sigma\left(A_{n}\right)\right)=\int_{A_{n}} f(x) d \mu(x)
$$

Since $\sigma$ is injective on $A$, we have,

$$
\mu(\sigma(A))=\sum_{n=1}^{\infty} \mu\left(\sigma\left(A_{n}\right)\right)=\sum_{n=1}^{\infty} \int_{A_{n}} f(x) d \mu(x)=\int_{A} f(x) d \mu(x) .
$$


Therefore $\mu$ is $f$-conformal.

Lemma 6.3.19. Let $\sigma: X \rightarrow X$ be a local homeomorphism. Let $n \in \mathbb{N}^{*}, x \in X$. Then there exists an open neighborhood $U$ of $x$ such that for $j=1, \ldots, n$,

$$
\left.\sigma\right|_{\sigma^{j-1}(U)}: \sigma^{j-1}(U) \rightarrow \sigma^{j}(U) \text { is a homeomorphism and } \sigma^{j}(U) \text { is open. }
$$

Proof. We prove this by induction. Let $n=1$. There exists an open neighborhood $U$ of $x$ such that $\sigma(U)$ is open and $\left.\sigma\right|_{U}: U \rightarrow \sigma(U)$ is a homeomorphism. Then the result holds for $n=1$.

Now assume the result holds for $n \geq 1$. Since $\sigma$ is a local homeomorphism, there exists an open neighborhood $W$ of $\sigma^{n}(x)$ in $\sigma^{n}(U)$ such that $\sigma(W)$ is open and $\left.\sigma\right|_{W}: W \rightarrow \sigma(W)$ is a homeomorphism.

Let $V=\left.\sigma\right|_{U} ^{-n}(W) . \quad V$ is an open neigborhood of $x$ in $U$. Let $j=1, \ldots, n$. Note that $\left.\sigma\right|_{U} ^{j}: U \rightarrow \sigma^{j}(U)$ is a homeomorphism. Since $V \subset U$, then $\sigma^{j}(V)$ is open and $\sigma^{j}(V) \subset \sigma^{j}(U)$. Hence $\left.\sigma\right|_{\sigma^{j-1}(V)}: \sigma^{j-1}(V) \rightarrow \sigma^{j}(V)$ is a homeomorphism for $i=1, \ldots, n$.

Note that $\sigma^{n+1}(V)=\sigma\left(\left.\left.\sigma\right|_{U} ^{n} \circ \sigma\right|_{U} ^{-n}(W)\right)=\sigma(W)$. Then $\sigma^{n+1}(V)$ is open. It follows that $\left.\sigma\right|_{\sigma^{n}(V)}: \sigma^{n}(V) \rightarrow \sigma^{n+1}(V)$ is a homeomorphism, therefore the result holds for $n+1$.

Remark 6.3.20. Let $x, y \in X$ such that $\sigma^{n}(x)=\sigma^{m}(y)$ for $n, m \in \mathbb{N}$. Assume $A$ is an open neigborhood of $x$ such that $(6.12)$ holds for $j=1, \ldots, n$, replacing $U$ by $A$. By the same argument, suppose there exists $B$, an open neigborhood of $y$ such that 6.12 holds for $j=1, \ldots, m$. Then we can assume without loss of generality that $\sigma^{n}(A)=\sigma^{m}(B)$.

In fact, let $V=\sigma^{n}(A) \cap \sigma^{m}(B)$. $V$ is an open neigborhood of $\sigma^{n}(x)$. Let $A_{0}=\left.\sigma\right|_{A} ^{-n}(V)$, $B_{0}=\left.\sigma\right|_{B} ^{-m}(V)$. Clearly $A_{0}$ and $B_{0}$ are open neigborhoods of $x, y$, respectively. Then, for $j=1, \ldots, n, 6.12$ holds for $A_{0}$, since $\sigma^{j-1}\left(A_{0}\right) \subset \sigma^{j-1}(A)$ and $\sigma^{j}\left(A_{0}\right) \subset \sigma^{j}(A)$ are open sets. Analogously 6.12 holds for $B_{0}$.

Moreover,

$$
\sigma^{n}\left(A_{0}\right)=\sigma^{n}\left(\left.\sigma\right|_{A} ^{-n}(V)\right)=V=\sigma^{m}\left(\left.\sigma\right|_{B} ^{-m}(V)\right)=\sigma^{m}\left(B_{0}\right)
$$


Theorem 6.3.21. Let $\beta \in \mathbb{R}$. A measure $\mu$ on $\mathcal{G}^{(0)}$ is $e^{\beta F}$-conformal if, and only if, $\mu$ is quasi-invariant with Radon-Nikodym derivative $e^{-\beta c_{F}}$.

Proof. Assume $\mu$ is $e^{\beta F}$-conformal.

Let $\mathcal{U} \subset \mathcal{G}$ be an open bisection. Let $T: r(\mathcal{U}) \rightarrow s(\mathcal{U})$ be the homeomorphism defined by $T=\left.\left.s\right|_{\mathcal{U}} \circ r\right|_{\mathcal{U}} ^{-1}$. Given $y \in s(\mathcal{U})$, let $h_{y}=\left.s\right|_{\mathcal{U}} ^{-1}(y)=(x, k, y)$. There are $A, B \subset X$ open sets, $n, m \in \mathbb{N}$ such that $h_{y} \in \mathcal{U}_{A, B}^{n, m} \subset \mathcal{U}$.

By Lemma 6.3.19 there exists an open neigborhood of $x, A_{0} \subset A$, such that for $j=$ $1, \ldots, n$,

$$
\left.\sigma\right|_{\sigma^{j-1}\left(A_{0}\right)}: \sigma^{j-1}\left(A_{0}\right) \rightarrow \sigma^{j}\left(A_{0}\right) \text { is a homeomorphism and } \sigma^{j}\left(A_{0}\right) \text { is open. }
$$

By the same argument, there exists an open neigborhood $B_{0}$ of $y$, with $B_{0} \subset B$, such that for $j=1, \ldots, m$,

$$
\left.\sigma\right|_{\sigma^{j-1}\left(B_{0}\right)}: \sigma^{j-1}\left(B_{0}\right) \rightarrow \sigma^{j}\left(B_{0}\right) \text { is a homeomorphism and } \sigma^{j}\left(B_{0}\right) \text { is open. }
$$

We can assume $\sigma^{n}\left(A_{0}\right)=\sigma^{m}\left(B_{0}\right)$ without loss of generality. Then $h_{y} \in \mathcal{U}_{A_{0}, B_{0}}^{n, m} \subset \mathcal{U}_{A, B}^{n, m}$.

Note that $s\left(\mathcal{U}_{A_{0}, B_{0}}^{n, m}\right)=B_{0}$ and $r\left(\mathcal{U}_{A_{0}, B_{0}}^{n, m}\right)=A_{0}$. In fact, since $\left.\sigma\right|_{A_{0}} ^{n},\left.\sigma\right|_{B_{0}} ^{m}$ are homeomorphisms, it follows that

$$
\begin{aligned}
\mathcal{U}_{A_{0}, B_{0}}^{n, m} & =\left\{\left(x^{\prime}, n-m, y^{\prime}\right): x^{\prime} \in A_{0}, y^{\prime} \in B_{0}, \sigma^{n}\left(x^{\prime}\right)=\sigma^{m}\left(y^{\prime}\right)\right\} \\
& =\left\{\left(\left.\sigma\right|_{A_{0}} ^{-n}\left(\left.\sigma\right|_{B_{0}} ^{m}\left(y^{\prime}\right)\right), n-m, y^{\prime}\right): y^{\prime} \in B_{0}\right\} \\
& =\left\{\left(x^{\prime}, n-m,\left.\sigma\right|_{B_{0}} ^{-m}\left(\left.\sigma\right|_{A_{0}} ^{n}\left(x^{\prime}\right)\right)\right): x^{\prime} \in A_{0}\right\} .
\end{aligned}
$$

Moreover, $\left.T\right|_{A_{0}}=\left.\left.\sigma\right|_{B_{0}} ^{-m} \circ \sigma\right|_{A_{0}} ^{n}$. Note that

$$
\begin{aligned}
\left.\left.\sigma\right|_{B_{0}} ^{-m} \circ \sigma\right|_{A_{0}} ^{n} & =\left.\left.\left.\left.\sigma\right|_{B_{0}} ^{-1} \circ \sigma\right|_{\sigma\left(B_{0}\right)} ^{-1} \circ \ldots \circ \sigma\right|_{\sigma^{m-1}\left(B_{0}\right)} ^{-1} \circ \sigma\right|_{A_{0}} ^{n} \\
& =\left.\left.\left.\left.\left.\sigma\right|_{B_{0}} ^{-m} \circ \sigma\right|_{\sigma^{n-1}\left(A_{0}\right)} \circ \sigma\right|_{\sigma^{n-2}\left(A_{0}\right)} \circ \ldots \sigma\right|_{\sigma^{1}\left(A_{0}\right)} \circ \sigma\right|_{A_{0}}
\end{aligned}
$$


Given $j=0, \ldots, n+m$, let

$$
T_{j}= \begin{cases}\left.\left.\sigma\right|_{B_{0}} ^{-m} \circ \sigma\right|_{\sigma^{j}\left(A_{0}\right)} ^{n-j}, & \text { if } 0 \leq j \leq n, \\ \left.\sigma\right|_{B_{0}} ^{-m-n+j}, & \text { if } n \leq j \leq n+m\end{cases}
$$

Then $T_{0}=\left.T\right|_{A_{0}}$ and $T_{n+m}=\left.i d\right|_{B_{0}}$. Note that $T_{j}: \sigma^{j}\left(A_{0}\right) \rightarrow B_{0}$ if $j \leq n$ and $T_{j}$ : $\sigma^{n+m-j}\left(B_{0}\right) \rightarrow B_{0}$ if $j \geq n$. Moreover,

- if $0 \leq j \leq n-1$

$$
T_{j}=\left.\left.\sigma\right|_{B_{0}} ^{-m} \circ \sigma\right|_{\sigma^{j}\left(A_{0}\right)} ^{n-j}=\left.\left.\left.\sigma\right|_{B_{0}} ^{-m} \circ \sigma\right|_{\sigma^{j+1}\left(A_{0}\right)} ^{n-j-1} \circ \sigma\right|_{\sigma^{j}\left(A_{0}\right)}=\left.T_{j+1} \circ \sigma\right|_{\sigma^{j}\left(A_{0}\right)} .
$$

Then $T_{j+1}=\left.T_{j} \circ \sigma\right|_{\sigma^{j}\left(A_{0}\right)} ^{-1}$. Also,

$$
\begin{aligned}
T_{j}^{-1} y & =\left.\left.\sigma\right|_{\sigma^{j}\left(A_{0}\right)} ^{j-n} \circ \sigma\right|_{B_{0}} ^{m}(y) \\
& =\left.\left(\left.\sigma\right|_{\sigma^{j}\left(A_{0}\right)} ^{n-j}\right)^{-1} \circ \sigma\right|_{A_{0}} ^{n}(x) \\
& =\left.\left(\left.\left.\sigma\right|_{A_{0}} ^{n} \circ \sigma\right|_{A_{0}} ^{-j}\right)^{-1} \circ \sigma\right|_{A_{0}} ^{n}(x) \\
& =\left.\left.\left.\sigma\right|_{A_{0}} ^{j} \circ \sigma\right|_{A_{0}} ^{-n} \circ \sigma\right|_{A_{0}} ^{n}(x) \\
& =\left.\sigma\right|_{A_{0}} ^{j}(x) .
\end{aligned}
$$

Let $B_{1} \subset B_{0}$ be measurable. Then,

$$
\begin{aligned}
T_{j+1_{*}} \mu\left(B_{1}\right) & =\left.T_{j_{*}} \sigma\right|_{\sigma^{j}\left(A_{0}\right)_{*}} ^{-1} \mu\left(B_{1}\right) \\
& =\left.\sigma\right|_{\sigma^{j}\left(A_{0}\right)_{*}} ^{-1} \mu\left(T_{j}^{-1}\left(B_{1}\right)\right) \\
& =\mu\left(\sigma\left(T_{j}^{-1}\left(B_{1}\right)\right)\right) .
\end{aligned}
$$

$T_{j}^{-1}\left(B_{1}\right)$ is measurable, since $T_{j}$ is continuous. Since $T_{j}^{-1}\left(B_{1}\right) \subset \sigma^{j}\left(A_{0}\right), \sigma$ is injective 
on $\sigma^{j}\left(A_{0}\right)$ and $\mu$ is $e^{\beta F}$-conformal, we have

$$
\begin{aligned}
\mu\left(\sigma\left(T_{j}^{-1}\left(B_{1}\right)\right)\right) & =\int_{T_{j}^{-1}\left(B_{0}\right)} e^{\beta F(u)} d \mu(u) \\
& =\int_{T_{j}^{-1}\left(B_{0}\right)} e^{\beta F\left(T_{j}^{-1} T_{j} u\right)} d \mu(u) \\
& =\int_{B_{0}} e^{\beta F\left(T_{j}^{-1} u\right)} d T_{j_{*}} \mu(u) \quad \text { by } 2.2 \text { on page } 15
\end{aligned}
$$

Then

$$
\frac{T_{j+1_{*}} \mu}{T_{j_{*}} \mu}(y)=e^{\beta F\left(T_{j}^{-1} y\right)}=e^{\beta F\left(\sigma^{j}(x)\right)} .
$$

Therefore,

$$
\frac{T_{j_{*}} \mu}{T_{j+1_{*}} \mu}(y)=e^{-\beta F\left(\sigma^{j}(x)\right)} .
$$

- if $n \leq j \leq n+m-1$,

$$
\begin{aligned}
T_{j} & =\left.\sigma\right|_{B_{0}} ^{-m-n+j} \\
& =\left(\left.\sigma\right|_{B_{0}} ^{m+n-j}\right)^{-1} \\
& =\left(\left.\left.\sigma\right|_{\sigma^{m+n-j-1}\left(B_{0}\right)} ^{m+\sigma}\right|_{B_{0}} ^{m+n-j-1}\right)^{-1} \\
& =\left.\left(\left.\sigma\right|_{B_{0}} ^{m+n-j-1}\right)^{-1} \circ \sigma\right|_{\sigma^{m+n-j-1}\left(B_{0}\right)} ^{-1} \\
& =\left.\left(\left.\sigma\right|_{B_{0}} ^{-m-n+j+1}\right)^{-1} \circ \sigma\right|_{\sigma^{m+n-j-1}\left(B_{0}\right)} ^{-1} \\
& =\left.T_{j+1} \circ \sigma\right|_{\sigma^{m+n-j-1}\left(B_{0}\right)} ^{-1} .
\end{aligned}
$$

Moreover,

$$
T_{j}^{-1} y=\left.\sigma\right|_{B_{0}} ^{m+n-j}(y)=\sigma^{m+n-j}(y)
$$


Let $B_{1} \subset B_{0}$ measurable. Then,

$$
\begin{aligned}
T_{j *} \mu\left(B_{1}\right) & =\left.T_{j+1_{*}} \sigma\right|_{\sigma^{m+n-j-1}\left(B_{0}\right)_{*}} ^{-1} \mu\left(B_{1}\right) \\
& =\left.\sigma\right|_{\sigma^{m+n-j-1}\left(B_{0}\right)_{*}} ^{-1} \mu\left(T_{j+1}^{-1}\left(B_{1}\right)\right) \\
& =\mu\left(\sigma\left(T_{j+1}^{-1}\left(B_{1}\right)\right)\right) .
\end{aligned}
$$

Note that $T_{j+1}^{-1}\left(B_{1}\right)$ is measurable by continuity of $T_{j+1}$. Since

$$
T_{j+1}^{-1}\left(B_{1}\right) \subset \sigma^{m+n-j-1}\left(B_{0}\right),
$$

$\sigma$ is injective on $\sigma^{m+n-j-1}\left(B_{0}\right)$ and $\mu$ is $e^{\beta F}$-conformal, we have

$$
\begin{aligned}
\mu\left(\sigma\left(T_{j+1}^{-1}\left(B_{1}\right)\right)\right) & =\int_{T_{j+1}^{-1}\left(B_{1}\right)} e^{\beta F(u)} d \mu(u) \\
& =\int_{T_{j+1}^{-1}\left(B_{1}\right)} e^{\beta F\left(T_{j+1}^{-1} T_{j+1} u\right)} d \mu(u) \\
& =\int_{B_{1}} e^{\beta F\left(T_{j+1}^{-1} u\right)} d T_{j+1_{*}} \mu(u) \quad \text { by } 2.2 \text { on page } 15
\end{aligned}
$$

Then,

$$
\frac{d T_{j_{*}} \mu}{d T_{j+1_{*}} \mu}(y)=e^{\beta F\left(T_{j+1}^{-1}(y)\right)}=e^{\sigma^{m+n-j-1}(y)} .
$$

Therefore,

$$
\begin{aligned}
\frac{d T_{*} \mu}{d \mu}(y) & =\frac{d T_{0 *} \mu}{d T_{m+n_{*}} \mu}(y) \\
& =\frac{d T_{0 *} \mu}{d T_{1 *} \mu}(y) \frac{d T_{1_{*}} \mu}{d T_{2 *} \mu}(y) \ldots \frac{d T_{n+m-1_{*}} \mu}{d T_{n+m_{*}} \mu}(y) \\
& =\left(\prod_{j=0}^{n-1} \frac{d T_{j_{*}} \mu}{d T_{j+1_{*}} \mu}(y)\right)\left(\prod_{j=n}^{n+m-1} \frac{d T_{j_{*}} \mu}{d T_{j+1_{*}} \mu}(y)\right)
\end{aligned}
$$




$$
\begin{aligned}
& \left.=\left(\prod_{j=0}^{n-1} e^{-\beta F\left(\sigma^{j}(x)\right)}\right)\left(\prod_{j=n}^{n+m-1} e^{\beta F\left(\sigma^{m+n-j-1}(y)\right)}\right) \quad \text { from } 6.13 \text { and } 6.14\right) \\
& =\left(\prod_{j=0}^{n-1} e^{-\beta F\left(\sigma^{j}(x)\right)}\right)\left(\prod_{j=0}^{m-1} e^{\beta F\left(\sigma^{m-j-1}(y)\right)}\right)
\end{aligned}
$$

Making the change of variables $j \mapsto m-j-1$ in the second product, we have

$$
\begin{aligned}
& =\left(\prod_{j=0}^{n-1} e^{-\beta F\left(\sigma^{j}(x)\right)}\right)\left(\prod_{j=0}^{m-1} e^{\beta F\left(\sigma^{j}(y)\right)}\right) \\
& =\exp \left(-\beta \sum_{j=0}^{n-1} F\left(\sigma^{j}(x)\right)+\beta \sum_{j=0}^{m-1} F\left(\sigma^{j}(y)\right)\right) \\
& =\exp \left(-\beta\left(\sum_{j=0}^{n-1} F\left(\sigma^{j}(x)\right)-\sum_{j=0}^{m-1} F\left(\sigma^{j}(y)\right)\right)\right) \\
& =e^{-\beta c_{F}(x, k, y)} \\
& =e^{-\beta c_{F}\left(h_{y}\right)}
\end{aligned}
$$

Since $y \in s(\mathcal{U})$ is arbitrary, the equality holds for every $y \in s(\mathcal{U})$. $\mathcal{U}$ is any open bisection. Therefore, by Lemma 5.3.8, $\mu$ is quasi-invariant with Radon-Nikodym derivative $e^{-\beta c_{F}}$.

Conversely, assume $\mu$ is quasi-invariant with Radon-Nikodym derivative $e^{-\beta c_{F}}$.

Let $A \subset X$ be an open set such that $\left.\sigma\right|_{A}$ is invertible. $\mathcal{U}_{\sigma(A), A}^{0,1}$ is an open bisection. In fact,

$$
\begin{aligned}
\mathcal{U}_{\sigma(A), A}^{0,1} & =\{(x,-1, y): x \in \sigma(A), y \in A, x=\sigma(y)\} \\
& =\{(\sigma(y),-1, y): y \in A\}
\end{aligned}
$$

Since $\sigma$ is injective on $A$, it follows that $r, s$ are injective on $\mathcal{U}_{\sigma(A), A}^{0,1}$. Moreover, $A=s\left(\mathcal{U}_{\sigma(A), A}^{0,1}\right)$, $\sigma(A)=r\left(\mathcal{U}_{\sigma(A), A}^{0,1}\right)$, and $T: \sigma(A) \rightarrow A$ is given by $T=\left.\sigma\right|_{A} ^{-1}$. From Lemma 5.3.8.

$$
\begin{aligned}
\frac{d T_{*} \mu}{d \mu}(y) & =e^{-\beta c_{F}\left(h_{y}\right)} \\
& =\exp \left(-\beta c_{F}(\sigma(y),-1, y)\right)
\end{aligned}
$$




$$
\begin{aligned}
& =\exp \left(-\beta\left(\sum_{j=0}^{0} F\left(\sigma^{j}(\sigma(y))-\sum_{j=0}^{1} F\left(\sigma^{j}(y)\right)\right)\right)\right. \\
& =\exp (-\beta[F(\sigma(y))-F(y)-F(\sigma(y))]) \\
& =e^{\beta F(y)}
\end{aligned}
$$

Hence,

$$
\mu(\sigma(A))=\mu\left(\left.\sigma\right|_{A}(A)\right)=\mu\left(T^{-1}(A)\right)=T_{*} \mu(A)=\int_{A} e^{\beta F(y)} d \mu(y)
$$

It follows from Lemma 6.3 .18 that $\mu$ is $e^{\beta F}$-conformal.

Remark 6.3.22. Let $\beta \neq 0$. Given a $e^{\beta F}$-conformal measure, it follows from Theorem 6.3.21 that $\mu$ is quasi-invariant with Radon-Nikodym derivative $e^{-\beta c_{F}}$. From Remark 4.1 .9 we have

$$
-\beta c_{F}(g)=0 \text { for } \mu \text {-a.e. } x \in G^{(0)} \text { and all } g \in G_{x}^{x} \text {, }
$$

then

$$
c_{F}(g)=0 \text { for } \mu \text {-a.e. } x \in G^{(0)} \text { and all } g \in G_{x}^{x} \text {, }
$$

We can show this fact for extremal $e^{\beta F}$-conformal probability measures using the properties of $\mathcal{G}$ when the set of periodic points in $X$ is countable.

Let $\mu$ be an extremal $e^{\beta F}$-conformal probability measure. From Lemma 6.3.3 and Lemma 6.3.9, the measure falls in one of these cases: $\mu$ is continuous; $\mu$ is purely atomic and supported on a periodic orbit; or $\mu$ is purely atomic and supported on an aperiodic orbit.

Suppose $\mu$ is continuous. Since the set of periodic points is countable, it follows that for $\mu$-a.e. $y, \mathcal{G}_{y}^{y}=\{(y, 0, y)\}$. However, $c_{F}(y, 0, y)=0$. Then the result holds for $\mu$ continuous.

If $\mu$ is purely atomic and supported on an aperiodic orbit $\mathcal{O}(z)$, then $\mathcal{G}_{y}^{y}=\{(y, 0, y)\}$ for 
every $y \in \mathcal{O}(z)$. Then 6.16 holds.

If $\mu$ is purely atomic and supported on a periodic orbit $\mathcal{O}(x)$ for a periodic point $x$ with minimum period $p$, it follows from 6.3 .8 that

$$
\sum_{i=0}^{p-1} F\left(\sigma^{i}(x)\right)=0
$$

Given $y \in \mathcal{O}(x), \mathcal{G}_{y}^{y}=\{(y, k p, y): k \in \mathbb{Z}\}$ from Lemma 6.2.10. From Lemma 6.3.16, we have $c_{F}(y, k p, y)=0$ for every $k \in \mathbb{Z}$. Then (6.16) holds.

Hence, the result follows for every extremal $e^{\beta F}$-conformal probability measure.

\subsection{KMS States on the Renault-Deaconu Groupoid}

In this section we find all extremal KMS states on $C^{*}(\mathcal{G})$ using Neshveyev's Theorems. Since the continuous $\mathbb{R}$-valued 1-cocycle on $\mathcal{G}$ is given by $c_{F}$ as in Definition 6.3.11, we fix the dynamics $\tau$ on $C^{*}(\mathcal{G})$, given by $\tau_{t}(f)(g)=e^{i t c_{F}(g)} f(g)$.

We will show that all extremal KMS states are $\phi_{m}, \phi_{m_{z}}, \phi_{x}^{\lambda}$, defined below.

Let $m$ be an extremal $e^{\beta F}$-conformal non-atomic probability measure on $X$. We define $\phi_{m}$ by

$$
\phi_{m}(f)=\int_{X} f(y, 0, y) d m(y)
$$

where $f \in C_{c}(\mathcal{G})$.

Given an aperiodic $\beta$-summable point $z$, by Proposition $6.3 .17 m_{z}$ is an extremal $e^{\beta F_{-}}$ conformal probability measure. We define $\phi_{m_{z}}$ by

$$
\phi_{m_{z}}(f)=\int_{X} f(y, 0, y) d m_{z}(y)
$$

with $f \in C_{c}(\mathcal{G})$. 
Given a periodic point $x$ with period $p$ with $x$ satisfying the conditions of Proposition 6.3.10, $\lambda \in \mathbb{C}$ with $|\lambda|=1$, we define $\phi_{x}^{\lambda}$ by

$$
\phi_{x}^{\lambda}(f)=\int_{X} \sum_{k \in \mathbb{Z}} \lambda^{k} f(y, k p, y) d m_{x}(y)
$$

for $f \in C_{c}(\mathcal{G})$.

Lemma 6.4.1. Let $x \in \mathcal{G}^{(0)}$. Given $g_{1}, g_{2} \in \mathcal{G}_{x}^{x}, u_{g_{1}} \cdot u_{g_{2}}=u_{g_{1} g_{2}}$. As a consequence, $C^{*}\left(\mathcal{G}_{x}^{x}\right)$ is a commutative $\mathrm{C}^{*}$-algebra with identity $u_{x}$.

Proof. Let $g \in \mathcal{G}$, then

$$
\begin{aligned}
\left(u_{g_{1}} \cdot u_{g_{2}}\right)(g) & =\sum_{a b=g} u_{g_{1}}(a) u_{g_{2}}(b) \\
& = \begin{cases}1 & \text { if } g_{1} g_{2}=g \\
0 & \text { otherwise }\end{cases} \\
& =u_{g_{1} g_{2}}(g) .
\end{aligned}
$$

Lemma 6.4.2. Let $x \in X, y \in \mathcal{O}(x), h \in \mathcal{G}_{y}^{x}$. There exists an $*$-isomorphism $P: C^{*}\left(\mathcal{G}_{y}^{y}\right) \rightarrow$ $C^{*}\left(\mathcal{G}_{x}^{x}\right)$ given by $P\left(u_{g}\right)=u_{h g h^{-1}}$. Moreover, $P$ is an isometry.

Proof. Let $P: C^{*}\left(\mathcal{G}_{y}^{y}\right) \rightarrow C^{*}\left(\mathcal{G}_{x}^{x}\right)$ be the linear map defined by $P\left(u_{g}\right)=u_{h g h^{-1}}$. Note that $P$ is invertible with inverse given by $P^{-1}: C^{*}\left(\mathcal{G}_{x}^{x}\right) \rightarrow C^{*}\left(\mathcal{G}_{y}^{y}\right)$, defined by $P^{-1}\left(u_{\widetilde{g}}\right)=u_{h^{-1}} \tilde{g} h$.

First we show that $P$ is a homomorphism. Given $g_{1}, g_{2}, g \in \mathcal{G}_{y}^{y}$,

$$
\begin{gathered}
P\left(u_{g_{1}} \cdot u_{g_{2}}\right)=P\left(u_{g_{1} g_{2}}\right)=u_{h g_{1} g_{2} h^{-1}}=u_{h g_{1} h^{-1} h g_{2} h^{-1}}=u_{h g_{1} h^{-1}} \cdot u_{h g_{2} h^{-1}}=P\left(u_{g_{1}}\right) \cdot P\left(u_{g_{2}}\right), \\
P\left(u_{g}{ }^{*}\right)=P\left(u_{g^{-1}}\right)=u_{h g^{-1} h^{-1}}=u_{\left(h g h^{-1}\right)^{-1}}=u_{\left(h g h^{-1}\right)}{ }^{*}=P\left(u_{g}\right)^{*} .
\end{gathered}
$$


Since $P$ is linear, we have that $P$ is a $*$-homomorphism. So is $P^{-1}$ by the same arguments. Then $P$ is an $*$-isomorphism between $\mathrm{C}^{*}$-algebras and therefore $P$ is an isometry.

Remark 6.4.3. Lemma 6.4.2 can be generalized for a locally compact Hausdorff second countable étale groupoid $G$. Given $x, y \in G^{(0)}$ such that $G_{y}^{x} \neq \varnothing$, there exists an *isomorphism $P:: C^{*}\left(G_{y}^{y}\right) \rightarrow C\left(G_{x}^{x}\right)$ given by $P\left(u_{g}\right)=u_{h g h^{-1}}$.

Proposition 6.4.4. Suppose the set of periodic points in $X$ is countable. Let $\beta \in \mathbb{R} \backslash\{0\}$. Let $\phi$ be the $\mathrm{KMS}_{\beta}$-state corresponding to the pair $\left(\mu,\left\{\varphi_{y}\right\}_{y}\right)$. Then $\phi$ is extremal if and only if $\mu$ is an extremal $e^{\beta F}$-conformal measure and $\varphi_{y}$ is a character for $\mu$-a.e. $y$.

Proof. It follows from Theorem 5.3 .10 that $\mu$ is quasi-invariant with Radon-Nikodym derivative $e^{-\beta c_{F}}$. It follows from Theorem 6.3.21 that $\mu$ is $e^{\beta F}$-conformal.

Assume $\phi$ is extremal.

- Suppose $\mu$ is not extremal, then $\mu=t \mu_{1}+(1-t) \mu_{2}$ where $\mu_{1}, \mu_{2}$ are $e^{\beta F}$-conformal probability measures, $0<t<1$ and $\mu_{1} \neq \mu_{2}$.

Then $\mu_{1}$ and $\mu_{2}$ are quasi-invariant with Radon-Nikodym derivative $e^{-\beta c_{F}}$. Since $\mu_{1}, \mu_{2} \ll \mu$, conditions (ii) and (iii) of Theorem 5.3.10 are satisfied. Then $\left(\mu_{1},\left\{\varphi_{y}\right\}_{y}\right)$ ), $\left.\left(\mu_{2},\left\{\varphi_{y}\right\}_{y}\right)\right)$ correspond to the KMS states $\phi_{1}, \phi_{2}$, respectively. Note that $\phi_{1} \neq \phi_{2}$. Then

$$
\begin{aligned}
t \phi_{1}(f)+(1-t) \phi_{2}(f) & =t \int_{X} \sum_{g \in \mathcal{G}_{x}^{x}} f(g) \varphi_{x}\left(u_{g}\right) d \mu_{1}(x)+(1-t) \int_{X} \sum_{g \in \mathcal{G}_{x}^{x}} f(g) \varphi_{x}\left(u_{g}\right) d \mu_{2}(x) \\
& =\int_{X} \sum_{g \in \mathcal{G}_{x}^{x}} f(g) \varphi_{x}\left(u_{g}\right) d\left(t \mu_{1}+(1-t) \mu_{2}\right)(x) \\
& =\int_{X} \sum_{g \in \mathcal{G}_{x}^{x}} f(g) \varphi_{x}\left(u_{g}\right) d \mu(x) \\
& =\phi(f) .
\end{aligned}
$$

Then $\phi$ is not extremal. Contradiction. 
- Suppose $\mu$ is extremal and non-atomic. Let $I$ be the set aperiodic points in $X$. Then $\mu(I)=1$ by Lemma 6.2.9. Given $y \in I, \mathcal{G}_{y}^{y}=\{y\}$ by Lemma 6.2.10. Then every element in $C^{*}\left(\mathcal{G}_{y}^{y}\right)$ in the form $a u_{y}$, where $a \in \mathbb{C}$. In addition $\varphi_{y}\left(u_{y}\right)=1$ because $\varphi$ is a state and $u_{y}$ is the unit of $C^{*}\left(\mathcal{G}_{y}^{y}\right)$. Therefore $\varphi_{y}$ is a character.

- Assume $\mu$ is extremal and atomic. Then there exists an orbit $I$ with $\mu(I)=1$. Suppose there is $x \in I$ such that $\varphi_{x}$ is not a character. Then, by Lemma 6.4.1, there are states $\varphi_{x}^{(1)}, \varphi_{x}^{(2)}, t \in(0,1)$ such that $\varphi_{x}^{(1)} \neq \varphi_{x}^{(2)}$ and $\varphi_{x}=t \varphi_{x}^{(1)}+(1-t) \varphi_{x}^{(2)}$.

Define the field of states $\left\{\varphi_{y}^{(1)}\right\}_{y},\left\{\varphi_{y}^{(2)}\right\}_{y}$ by

$$
\varphi_{y}^{(i)}\left(u_{g}\right)= \begin{cases}\varphi_{y}\left(u_{g}\right) & \text { if } y \notin I \\ \varphi_{x}^{(i)}\left(u_{h g h^{-1}}\right) & \text { if } y \in I, \text { and } h \in G_{y}^{x} \text { arbitrary }\end{cases}
$$

for $i=1,2$. Note that $h g h^{-1}$ does not depend on the choice of $g$. In fact, given $y \in \mathcal{O}(x), g \in \mathcal{G}_{y}^{y}, h \in \mathcal{G}_{y}^{x}$, there exists $k, l \in \mathbb{N}$ such that $g=(y, k, y)$ and $h=(x, l, y)$. Then $h g h^{-1}=(x, k, x)$.

It is clear that $\varphi_{y}^{(i)}$ is a state for $y \notin I$. We will show that $\varphi_{y}^{(i)}$ is also a state when $y \in I$. Given $h \in \mathcal{G}_{y}^{x}$, let $P: C^{*}\left(\mathcal{G}_{y}^{y}\right) \rightarrow C^{*}\left(\mathcal{G}_{x}^{x}\right)$ be defined by $P\left(u_{g}\right)=u_{h g h^{-1}}$ for every $g \in \mathcal{G}_{y}^{y}$. It follows from Lemma 6.4.2 that $P$ is an isometry. Moreover, by definition of $\varphi_{y}^{(i)}$, we have $\varphi_{y}^{(i)}=\varphi_{x}^{(i)} \circ P . \varphi_{y}^{(i)}$ is linear and bounded because

$$
\left\|\varphi_{y}^{(i)}\right\| \leq\left\|\varphi_{x}^{(i)}\right\|\|P\|=\left\|\varphi_{x}^{(i)}\right\|
$$

Since $\varphi_{x}^{(i)}=\varphi_{y}^{(i)} \circ P^{-1}$, we can show analogously that $\left\|\varphi_{x}^{(i)}\right\| \leq\left\|\varphi_{y}^{(i)}\right\|$. Then $\left\|\varphi_{y}^{(i)}\right\|=$ $\left\|\varphi_{x}^{(i)}\right\|=1$. Moreover,

$$
\varphi_{y}^{(i)}\left(u_{y}\right)=\varphi_{x}^{(i)}\left(u_{h y h^{-1}}\right)=\varphi_{x}^{(i)}\left(u_{h h^{-1}}\right)=\varphi_{x}^{(i)}\left(u_{x}\right)=1
$$

Note that $u_{y}$ is the unit of $C^{*}\left(\mathcal{G}_{y}^{y}\right)$. It follows from Theorem 5.1 .2 that $\varphi_{y}^{(i)}$ is a state. 
Since $\mu$ is atomic, $\left\{\varphi_{y}^{(i)}\right\}_{y}$ defines a $\mu$-measurable field of states by Remark 5.2.5. For $i=1,2$, let $\phi^{(i)}$ be the state defined by $\left(\mu,\left\{\varphi_{y}^{(i)}\right\}_{y}\right)$. We will show that $\phi^{(i)}$ is a KMS state. In order to prove this, we have show that properties (i)-(iii) in Theorem 5.3.10 hold.

We already know that $\mu$ is quasi-invariant with Radon-Nikodym derivative $e^{-\beta c_{F}}$, so property (i) holds.

We will prove property (ii). Let $y \in I$, then there exists $k \in \mathcal{G}_{y}^{x}$. Let $h \in \mathcal{G}_{y}$. Then $r(k) \in I$ and $k h^{-1} \in \mathcal{G}_{r(h)}^{x}$. For every $g \in \mathcal{G}_{y}^{y}$, we have

$$
\begin{aligned}
& \varphi_{r(h)}^{(i)}\left(u_{h g h^{-1}}\right)=\varphi_{x}^{(i)}\left(u_{k h^{-1}\left(h g h^{-1}\right) h k^{-1}}\right), \quad \text { by definition of } \varphi_{r(h)}^{(i)} \text {, } \\
& =\varphi_{x}^{(i)}\left(u_{k g k^{-1}}\right) \\
& =\varphi_{y}^{(i)}\left(u_{y}\right) \text {, by definition of } \varphi_{y}^{(i)} \text {. }
\end{aligned}
$$

Then property (ii) holds.

For $\mu$-a.e. $y \in X$, all $g \in \mathcal{G}_{y}^{y}$, we have $c_{F}(g)=0$ by Remark 6.3.22. Then property (iii) holds.

By definition of $\varphi_{y}^{(i)}, \varphi_{y}\left(u_{g}\right)=t \varphi_{y}^{(1)}\left(u_{g}\right)+(1-t) \varphi_{y}^{(2)}\left(u_{g}\right)$ if $y \notin I$. If $y \in I$, there exists $h \in \mathcal{G}_{y}^{x}$. Then,

$$
\begin{aligned}
\varphi_{y}\left(u_{g}\right) & =\varphi_{r(h)}\left(u_{h g h^{-1}}\right) \\
& =\varphi_{x}\left(u_{h g h^{-1}}\right) \\
& =t \varphi_{x}^{(1)}\left(u_{h g h^{-1}}\right)+(1-t) \varphi_{x}^{(2)}\left(u_{h g h^{-1}}\right) \\
& =t \varphi_{y}^{(1)}\left(u_{g}\right)+(1-t) \varphi_{y}^{(2)}\left(u_{g}\right) .
\end{aligned}
$$

Hence $\varphi_{y}=t \varphi_{y}^{(1)}+(1-t) \varphi_{y}^{(2)}$ and $\varphi_{y}^{(1)} \neq \varphi_{y}^{(2)}$ for every $y \in I$. By definition of $\left\{\varphi_{y}^{(i)}\right\}_{y}$, $\left(\mu,\left\{\varphi_{y}^{(i)}\right\}_{y}\right)$ defines a state $\phi^{(i)}, i=1,2$. Note that $\phi^{(1)} \neq \phi^{(2)}$. 
Then, for $f \in C_{c}(G)$,

$$
\begin{aligned}
\phi(f) & =\int_{X} \sum_{g \in \mathcal{G}_{y}^{y}} f(g) \varphi_{y}\left(u_{g}\right) d \mu(y) \\
& =\int_{X} \sum_{g \in \mathcal{G}_{y}^{y}} f(g)\left[t \varphi_{y}^{(1)}\left(u_{g}\right)+(1-t) \varphi_{y}^{(2)}\left(u_{g}\right)\right] d \mu(y) \\
& =t \int_{X} \sum_{g \in \mathcal{G}_{y}^{y}} f(g) \varphi_{y}^{(1)} d \mu(y)+(1-t) \int_{X} \sum_{g \in \mathcal{G}_{y}^{y}} f(g) \varphi_{y}^{(2)} d \mu(y) \\
& =t \phi^{(1)}(f)+(1-t) \phi^{(2)}(f) .
\end{aligned}
$$

Then $\phi$ is not extremal. Contradiction. Therefore $\mu$ is extremal and $\varphi_{y}$ is a character for $\mu$-a.e. $y$.

Conversely, suppose $\mu$ is extremal and $\varphi_{y}$ is a character for $\mu$-a.e. $y$. Suppose there exist KMS states $\phi^{(1)}, \phi^{(2)}, t \in(0,1)$ such that $\phi=t \phi^{(1)}+(1-t) \phi^{(2)}$ and each $\phi^{(i)}$ corresponds to the pair $\left(\mu_{i},\left\{\varphi_{y}^{(i)}\right\}_{y}\right)$. Since $X$ is clopen $\mathcal{G}$, we have for every $f \in C_{c}(X)$,

$$
\begin{aligned}
\phi(f) & =t \phi^{(1)}(f)+(1-t) \phi^{(2)}(f) \\
\int_{X} f(x) d \mu(x) & =\int_{X} f(x) d \mu_{1}(x)+(1-t) \int_{X} f(x) d \mu_{2}(x) .
\end{aligned}
$$

Then $\mu=t \mu_{1}+(1-t) \mu_{2}$. Since $\mu$ is extremal, we have $\mu_{1}=\mu_{2}=\mu$. Then, for every $f \in C_{c}(\mathcal{G})$,

$$
\begin{aligned}
\phi(f) & =t \phi^{(1)}(f)+(1-t) \phi^{(2)}(f) \\
& =t \int_{X} \sum_{g \in \mathcal{G}_{x}^{x}} f(g) \varphi_{x}^{(1)}\left(u_{g}\right) d \mu(x)+(1-t) \int_{X} \sum_{g \in \mathcal{G}_{x}^{x}} f(g) \varphi_{x}^{(2)}\left(u_{g}\right) d \mu(x) \\
& =\int_{X} \sum_{g \in \mathcal{G}_{x}^{x}} f(g)\left[t \varphi_{x}^{(1)}\left(u_{g}\right)+(1-t) \varphi_{x}^{(2)}\left(u_{g}\right)\right] d \mu(x) .
\end{aligned}
$$

Each $t \varphi_{x}^{(1)}+(1-t) \varphi_{x}^{(2)}$ is a state on $C^{*}\left(\mathcal{G}_{x}^{x}\right)$. Moreover, $\left\{t \varphi_{x}^{(1)}+(1-t) \varphi_{x}^{(2)}\right\}_{x}$ is a $\mu$-measurable field of states. Then the pair $\left(\mu,\left\{t \varphi_{x}^{(1)}+(1-t) \varphi_{x}^{(2)}\right\}_{x}\right)$ also defines $\phi$. It follows from Theorem 
5.2 .9 that

$$
\varphi_{x}=t \varphi_{x}^{(1)}+(1-t) \varphi_{x}^{(2)}, \quad \text { for } \mu \text {-a.e. } x
$$

Since $\varphi_{x}$ is a character $\mu$-a.e., it follows that $\varphi_{x}=\varphi_{x}^{(1)}=\varphi_{x}^{(2)}$ for $\mu$-a.e. $x$. Then, by Theorem 5.2.9. $\phi=\phi^{(1)}=\phi^{(2)}$. Therefore $\phi$ is extremal.

Lemma 6.4.5. Let $y \in X$ be aperiodic. There is a unique state $\varphi_{y}$ on $C^{*}\left(\mathcal{G}_{y}^{y}\right)$. In particular, $\varphi_{y}$ is a character.

Proof. Since $\mathcal{G}_{y}^{y}=\{y\}$, it follows that $C^{*}\left(\mathcal{G}_{y}^{y}\right)$ is isomorphic to $\mathbb{C}$. Hence there is a unique state on $C^{*}\left(\mathcal{G}_{y}^{y}\right)$, which is a character.

Lemma 6.4.6. Let $y \in X$ such that $\mathcal{O}(y)$ is periodic with period $p$. Let $\lambda \in \mathbb{C}$ such that $|\lambda|=1$. Define the linear functional $\varphi_{y}^{\lambda}$ on $C^{*}\left(\mathcal{G}_{y}^{y}\right)$ by $\varphi_{y}^{\lambda}\left(u_{(y, k p, y)}\right)=\lambda^{k}$. Then $\varphi_{y}^{\lambda}$ is a character on $C^{*}\left(\mathcal{G}_{y}^{y}\right)$. In fact, $\varphi_{y}^{\lambda}$ are the only characters defined on $C^{*}\left(\mathcal{G}_{y}^{y}\right)$.

Proof. Note that we can identify $\mathcal{G}_{y}^{y}$ with $\mathbb{Z}$ by the isomorphism $(y, k p, y) \mapsto k$. Moreover, $C^{*}(\mathbb{Z})$ is isomorphic to $C\left(S^{1}\right)$, the set of continuous functions on the complex unit circle. In fact, for $k \in \mathbb{Z}$, let $u_{k}: \mathbb{Z} \rightarrow \mathbb{C}$ be defined by

$$
u_{k}(l)= \begin{cases}1 & \text { if } l=k \\ 0 & \text { otherwise }\end{cases}
$$

and let $p_{k}: S^{1} \rightarrow \mathbb{C}$ be defined by $p_{k}(z)=z^{k}$.

There exists an isomorphism from $C^{*}(\mathbb{Z})$ to $C\left(S^{1}\right)$ given by $u_{k} \mapsto p_{k}$. Since $\left\{p_{k}\right\}_{k \in \mathbb{Z}}$ generates the commutative $\mathrm{C}^{*}$-algebra $C\left(S^{1}\right)$, it follows that all characters of $C\left(S^{1}\right)$ correspond to elements on the unit circle $S^{1}$. Hence, each character $\varphi$ on $C^{*}\left(\mathcal{G}_{y}^{y}\right)$ corresponds to a character $\lambda$ on $C\left(S^{1}\right)$ such that

$$
\varphi\left(u_{(y, k p, y)}\right)=p_{k}(\lambda)=\lambda^{k} .
$$




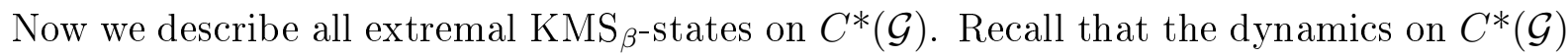
is given by $\tau_{t}(f)(g)=e^{i t c_{F}(g)} f(g)$.

Theorem 6.4.7. [26, Theorem 2.2] Let $\beta \in \mathbb{R} \backslash\{0\}$. Assume that the periodic points of $\sigma$ are countable. The extremal $\mathrm{KMS}_{\beta}$-states for $\tau$ are

1. States $\phi_{m}$, where $m$ is an extremal and continuous (non-atomic) $e^{\beta F}$-conformal Borel probability measure on $X$;

2. The states $\phi_{x}^{\lambda}$, where $\lambda \in \mathbb{C},|\lambda|=1$ and $x$ is periodic with minimum period $p$, such that

$$
\sum_{j=0}^{p-1} F\left(\sigma^{j}(x)\right)=0 \quad \text { and } \quad \sum_{n=1}^{\infty} \sum_{y \in Y_{n}} \exp \left(-\beta \sum_{j=0}^{n-1} F\left(\sigma^{j}(y)\right)\right)<\infty
$$

3. The states $\phi_{m_{z}}$ where $z$ is aperiodic and $\beta$-summable.

Proof. Let $\phi$ be an extremal $\mathrm{KMS}_{\beta}$-state corresponding to the pair $\left(\mu,\left\{\varphi_{y}\right\}_{y}\right)$. From Proposition 6.4.4 $\mu$ is an extremal $e^{\beta F}$-conformal probability measure, and $\varphi_{y}$ is a character for $\mu$-a.e. $y$.

It follows from Theorem 6.3.21 that $\mu$ is quasi-invariant with Radon-Nikodym derivative $e^{-\beta c_{F}}$. Since $\mu$ is extremal, $\mu$ is either atomic or non-atomic.

(i) Suppose $\mu=m$ is non-atomic or $\mu=m_{z}$ for $z$ aperiodic.

If $\mu=m_{z}$, then $z$ is $\beta$-summable by Proposition 6.3.17.

Let $\phi$ be an extremal $\mathrm{KMS}_{\beta^{-}}$-state corresponding to the pair $\left(m,\left\{\varphi_{y}\right\}_{y}\right)$. Define $I$ by

- the set of aperiodic points, i.e. points $X$ whose orbits are aperiodic, if $\mu$ is nonatomic. From Lemma 6.2.9 we have $m(I)=1$.

- $I=\mathcal{O}(z)$ if $\mu=m_{z}$. 
Then $\mu(I)=1$ and $y \in I$ is aperiodic for every $y \in I$. Then by Lemma 6.4.5, $\varphi_{y}$ defined by $\varphi_{y}\left(u_{y}\right)=1$ is the unique character defined on $C^{*}\left(\mathcal{G}_{y}^{y}\right)$.

Hence $\phi$ is defined by

$$
\phi(f)=\int_{X} \sum_{g \in \mathcal{G}_{y}^{y}} f(g) d \mu(y)=\int_{I} f(y, 0, y) d \mu(y)=\int_{X} f(y, 0, y) d \mu(y)
$$

Then $\phi=\phi_{m}$ if $\mu=m$ and $\phi=\phi_{m_{z}}$ if $\mu=m_{z}$.

(ii) Suppose $\mu=m_{x}$ with $x$ periodic with minimum period $p$.

From Proposition 6.3.10 conditions 6.17 must hold.

Let $I=\mathcal{O}(x)$. Let $\phi$ be an extremal $\mathrm{KMS}_{\beta}$-state with corresponding pair $\left(m_{x},\left\{\varphi_{y}\right\}_{y}\right)$. It follows from Proposition 6.4.4 and Lemma 6.4.6 that $\varphi_{x}=\varphi_{x}^{\lambda}$ for some $\lambda \in S^{1}$.

Let $y \in \mathcal{O}(x)$, then there exists $h=(x, l, y) \in \mathcal{G}$ for some $l \in \mathbb{Z}$. Let $g \in \mathcal{G}_{y}^{y}$, then $g=(y, k p, y)$ for some $k \in \mathbb{Z}$ by Lemma 6.2.10. Then applying property (iii) of Theorem 5.3 .10 ,

$$
\begin{aligned}
\varphi_{y}\left(u_{g}\right) & =\varphi_{r(h)}\left(u_{h g h^{-1}}\right) \\
& =\varphi_{x}\left(u_{(x, l, y)(y, k p, y)(y,-l, x)}\right) \\
& =\varphi_{x}\left(u_{(x, k p, x)}\right) \\
& =\varphi_{x}^{\lambda}\left(u_{(x, k p, x)}\right) \\
& =\lambda^{k} \\
& =\varphi_{y}^{\lambda}\left(u_{(y, k p, y)}\right) \\
& =\varphi_{y}^{\lambda}\left(u_{g}\right) .
\end{aligned}
$$

Then $\varphi_{y}=\varphi_{y}^{\lambda}$ for every $y \in I$. Then for $f \in C_{c}(G)$,

$$
\phi(f)=\int_{X} \sum_{g \in \mathcal{G}_{y}^{y}} f(g) \varphi_{y}\left(u_{g}\right) d m_{x}(y)
$$




$$
\begin{aligned}
& =\int_{I} \sum_{g \in \mathcal{G}_{y}^{y}} f(g) \varphi_{y}\left(u_{g}\right) d m_{x}(y) \\
& =\int_{I} \sum_{g \in \mathcal{G}_{y}^{y}} f(g) \varphi_{y}^{\lambda}\left(u_{g}\right) d m_{x}(y) \\
& =\int_{I} \sum_{k \in \mathbb{Z}} f(y, k p, y) \varphi_{y}^{\lambda}\left(u_{y, k p, y}\right) d m_{x}(y) \\
& =\int_{I} \sum_{k \in \mathbb{Z}} f(y, k p, y) \lambda^{k} d m_{x}(y) \\
& =\int_{X} \sum_{k \in \mathbb{Z}} f(y, k p, y) \lambda^{k} d m_{x}(y) \\
& =\phi_{x}^{\lambda}(f) .
\end{aligned}
$$

Hence every extremal KMS state has the form $\phi_{m}, \phi_{m_{z}}$ or $\phi_{x}^{\lambda}$.

Now we prove that $\phi_{m}, \phi_{m_{z}}, \phi_{x}^{\lambda}$ satisfying the conditions of the theorem always define extremal KMS states.

Note that, for an extremal $e^{\beta F}$-conformal probability measure $\mu$ on $X$, it follows from Remark 6.3.22 that for $\mu$-a.e. $x \in X$, all $g \in \mathcal{G}_{x}^{x}$, we have $c_{F}(g)=0$. Then in order to prove that a state defined by $\left(\mu,\left\{\varphi_{y}\right\}_{y}\right)$ is $\mathrm{KMS}_{\beta}$, we only need to show that property (ii) of Theorem 5.3 .10 holds.

- $\phi_{m}$

Let $m$ be an extremal and continuous $e^{\beta F}$-conformal Borel probability measure on $X$. Let $\left\{\varphi_{y}\right\}_{y \in X}$ be a family of states $\varphi_{y}$ on $C^{*}\left(\mathcal{G}_{y}^{y}\right)$.

Let $I$ be the set of aperiodic points. $m(X \backslash I)=0$ by Lemma 6.2.9. Let $f \in C_{c}(\mathcal{G})$. Given $y \in I, \mathcal{G}_{y}^{y}=\{y\}$. Then

$$
\sum_{g \in \mathcal{G}_{y}^{y}} f(g) \varphi\left(u_{g}\right)=f(y, 0, y)
$$

Thus $\left\{\varphi_{y}\right\}_{y}$ is a $m$-measurable field of states. Moreover, it follows from Lemma 6.4 .5 that $\varphi_{y}$ is a character for every $y \in I$. 
Now we show that $\left(m,\left\{\varphi_{y}\right\}_{y}\right)$ defines a KMS state. Let $y \in I$, then its orbit is aperiodic. Let $h \in \mathcal{G}_{y} \cdot g=y$ is the unique element in $\mathcal{G}_{y}^{y}$. Then

$$
\begin{aligned}
\varphi_{y}\left(u_{g}\right) & =\varphi_{y}\left(u_{y}\right)=1, \quad \text { and } \\
\varphi_{r(h)}\left(u_{h g h^{-1}}\right) & =\varphi_{r(h)}\left(u_{h y h^{-1}}\right)=\varphi_{r(h)}\left(u_{h h^{-1}}\right)=\varphi_{r(h)}\left(u_{r(h)}\right)=1 .
\end{aligned}
$$

Then property (ii) of Theorem 5.3.10 and, therefore, $\phi_{m}$ is a KMS state. Since $m$ is extremal and $\varphi_{y}$ is extremal for $m$-a.e. $y$, it follows from Proposition 6.4.4 that $\phi_{m}$ is extremal.

- $\phi_{m_{z}}$

This case is analogous to the proof for $m$ continuous if we define $I=\mathcal{O}(z)$. Note that $m_{z}$ is defined only if $z$ is $\beta$-summable by Proposition 6.3.17.

- $\phi_{x}^{\lambda}$

Let $x \in X$ be periodic with minimum period $p$ such that 6.17 holds. Then the extremal $e^{\beta F}$-conformal probability measure $m_{x}$ supported on $\mathcal{O}(x)$ exists by Proposition 6.3.10. Let $\left\{\varphi_{y}\right\}_{y \in X}$ be a family os states such that $\varphi_{y}=\varphi_{y}^{\lambda}$ for every $y \in \mathcal{O}(x)$. Then $\left\{\varphi_{y}\right\}_{y \in X}$ defines a $m_{x}$-measurable field of states. Moreover, $\varphi_{y}$ is a character for $\mu$-a.e. $y$ by Lemma 6.4.6.

Now we show that $\left(m_{x},\left\{\varphi_{y}\right\}_{y}\right)$ defines a KMS state, so we will show that property (ii) of Theorem 5.3.10 holds. Let $h \in \mathcal{G}_{x}$. There is $l \in \mathbb{Z}$, such that $h=(r(h), l, x)$. Let $g \in \mathcal{G}_{x}^{x}$. Then there exists $k \in \mathbb{Z}$ such that $g=(x, k p, x)$. Hence,

$$
\begin{aligned}
\varphi_{x}\left(u_{g}\right) & =\varphi_{x}\left(u_{(x, k p, x)}\right)=\lambda^{k}, \quad \text { and } \\
\varphi_{r(h)}\left(u_{h g h^{-1}}\right) & =\varphi_{r(h)}\left(u_{(r(h), l, x)(x, k p, x)(x,-l, r(h))}\right)=\varphi_{r(h)}\left(u_{(r(h), k p, r(h))}\right)=\lambda^{k} .
\end{aligned}
$$

Then $\left(m_{x},\left\{\varphi_{y}\right\}_{y}\right)$ defines a KMS state. Moreover, this state is extremal by Propo- 
sition 6.4.4. Given $f \in C_{c}(G)$, this state is defined by

$$
\begin{aligned}
\int_{X} \sum_{g \in \mathcal{G}_{y}^{y}} f(g) \varphi_{x}\left(u_{g}\right) d m_{x} & =\int_{I} \sum_{g \in \mathcal{G}_{y}^{y}} f(g) \varphi_{x}^{\lambda}\left(u_{g}\right) d m_{x} \\
& =\int_{I} \sum_{k \in \mathbb{Z}} f(y, k p, y) \varphi_{x}^{\lambda}\left(u_{(y, k p, y)}\right) d m_{x} \\
& =\int_{I} \sum_{k \in \mathbb{Z}} f(y, k p, y) \lambda^{k} d m_{x} \\
& =\int_{X} \sum_{k \in \mathbb{Z}} f(y, k p, y) \lambda^{k} d m_{x} \\
& =\phi_{x}^{\lambda}(f) .
\end{aligned}
$$

Therefore the extremal KMS states on $C^{*}(\mathcal{G})$ are precisely $\phi_{m}, \phi_{m_{z}}$ and $\phi_{x}^{\lambda}$.

Corollary 6.4.8. Let $\beta \in \mathbb{R} \backslash\{0\}$. Assume $F$ is positive on $X$. Then there exists a correspondence between the extremal $\mathrm{KMS}_{\beta}$ states and the extremal and continuous $e^{\beta F_{-}}$ conformal probability measures on $X$.

Proof. Let $m$ be an extremal and continuous $e^{\beta F}$-conformal probability measure on $X$. Then $\phi_{m}$ is a $\mathrm{KMS}_{\beta}$-state on $C^{*}(\mathcal{G})$ by Theorem 6.4 .7 .

Conversely, suppose $\phi$ is an extremal $\mathrm{KMS}_{\beta}$-state. We will see that $\phi$ is not in cases 2 and 3 of theorem Theorem 6.4.7,

2. Suppose $\phi=\phi_{x}^{\lambda}$ for some $\lambda \in \mathbb{C}$ with $|\lambda|=1$ and $x \in X$ periodic with minimum period $p>0$. Then

$$
\sum_{j=0}^{p-1} F\left(\sigma^{j}(x)\right)=0
$$

Contradiction, since $F$ assumes positive values.

3. Suppose $\phi=\phi_{m_{z}}$ for some $z \in X$ aperiodic. 
Define the sequence $\left\{y_{n}\right\}_{n \in \mathbb{N}}$ by $y_{n}=\sigma^{n}(z)$. Then

$$
\mathcal{F}\left(y_{n}\right)=-\sum_{j=0}^{n-1} F\left(\sigma^{j}(z)\right)=-\sum_{j=0}^{n-1} F\left(y_{j}\right)
$$

Then, for each $n, \mathcal{F}\left(y_{n+1}\right)<\mathcal{F}\left(y_{n}\right)$. Hence, $e^{-\beta \mathcal{F}\left(y_{n+1}\right)}>e^{-\beta \mathcal{F}\left(y_{n}\right)}$. So,

$$
\sum_{n=0}^{\infty} e^{-\beta \mathcal{F}\left(y_{n}\right)}=\infty
$$

Then

$$
\sum_{y \in \mathcal{O}(z)} e^{-\beta \mathcal{F}(y)}=\infty
$$

Therefore $z$ is not $\beta$-summable. Contradiction, since we assumed $\phi=\phi_{m_{z}}$.

Therefore $\phi=\phi_{m}$ for an extremal and continuous $e^{\beta F}$-conformal Borel probability measure on $X$. 


\section{Chapter 7}

\section{Concluding Remarks}

In this thesis we described KMS states on groupoid $\mathrm{C}^{*}$-algebras for locally compact Hausdorff second countable étale groupoids using Neshveyev's theorems. Then we studied a theorem due to Thomsen which characterizes the extremal KMS states for the Renault-Deaconu groupoid.

Neshveyev's theorem proved to be a useful tool to find an explicit formula for all KMS states. However, the proof of this theorem depends on the fact that the groupoid is étale. When the groupoid is not étale, it is possible to define groupoid $\mathrm{C}^{*}$-algebras which are similar

to the $\mathrm{C}^{*}$-algebras studied in this thesis. For instance, [10] defines the crossed product of a $\mathrm{C}^{*}$-algebra by a groupoid $G$ where this groupoid is locally compact Hausdorff and is endowed with a Haar system. This space is a closure of a space of continuous and compactly supported functions $f$ on $X$ such that, for every $x \in X, f(x)$ is an element of a $\mathrm{C}^{*}$-algebra. The operations in this space are analogous to the operations in a full $\mathrm{C}^{*}$-algebra. So, one challenge is to extend Neshveyev's theorem to non-étale groupoids.

Christensen [5] generalized the theorem of Neshveyev to describe KMS weights. This result can be applied to describe KMS weights for different groupoids. 


\section{Bibliography}

[1] Joseph Bak and Donald J. Newman. Complex Analysis. Springer, 2010.

[2] Ola Bratteli and Derek W. Robinson. Operator Algebras and Quantum Statistical Mechanics: Volume 1: $C^{*}$-and $W^{*}$-Algebras. Symmetry Groups. Decomposition of States. Springer-Verlag, 1979.

[3] Ola Bratteli and Derek W. Robinson. Operator Algebras and Quantum Statistical Mechanics. Vol. 2: Equilibrium states. Models in quantum statistical mechanics. SpringerVerlag, 1997.

[4] Jonathan H. Brown. Proper Actions of Groupoids on $C^{*}$-algebras. PhD thesis, Dartmouth College, Hanover, New Hampshire, May 2009.

[5] Johannes Christensen. KMS Weights on Groupoid $C^{*}$-algebras: With an Emphasis on Graph $C^{*}$-algebras. PhD thesis, Aarhus University, Aarhus, Denmark, July 2018.

[6] Donald L. Cohn. Measure Theory. Birkhäuser, 1980.

[7] Claire Debord and Jean-Marie Lescure. Index theory and Groupoids. In Hernan Ocampo, Eddy Pariguan, and Sylvie Paycha, editors, Geometric and Topological Methods for Quantum Field Theory, pages 86-158. Cambridge University Press, 2010.

[8] Manfred Denker and Mariusz Urbański. On the Existence of Conformal Measures. Transactions of the American Mathematical Society, 328(2):563-587, 1991. 
[9] Rodrigo S. Frausino. Groupoid C*-algebras, Conformal Measures and Phase Transitions. Master's thesis, University of São Paulo, São Paulo, May 2018.

[10] Geoff Goehle. Groupoid Crossed Products. PhD thesis, Dartmouth College, Hanover, New Hampshire, May 2009.

[11] Chaim S. Hönig. Aplicações da Topologia à Análise. Instituto de Matemática Pura e Aplicada, CNPq, 1976.

[12] Roy A. Johnson. Atomic and Nonatomic Measures. Proceedings of the American Mathematical Society, 25(3):650-655, 1970.

[13] Alexander Kechris. Classical descriptive set theory. Springer-Verlag, 1995.

[14] Mauro S. de F. Marques. Teoria da medida. UNICAMP, 2009.

[15] Paul S. Muhly and Dana P. Williams. Renault's equivalence theorem for groupoid crossed products. New York Journal of Mathematics, 3:1-87, 2008.

[16] Gerard J. Murphy. $C^{*}$-Algebras and Operator Theory. Academic press, 1990.

[17] Sergey Neshveyev. KMS States on the $C^{*}$-Algebras of Non-Principal Groupoids. Journal of Operator Theory, 70(2):513-530, 2013.

[18] Ian F. Putnam. Lecture Notes on $C^{*}$-Algebras, January 2016.

[19] Clive Reis. Abstract Algebra: An Introduction to Groups, Rings and Fields. World Scientific Publishing Company, 2011.

[20] Jean Renault. A Groupoid Approach to $C^{*}$-Algebras, volume 793. Springer-Verlag, 1980.

[21] Jadevilson C. Ribeiro and Rafael P. Lima. Funções holomorfas a valores em um $\mathbb{C}$ espaço vetorial topológico. https : / / www . ime . usp . br / cordaro / wp content/uploads/2017/09/Jadevilson-Rafael.pdf, 2017. Final project for a course on Locally Convex Spaces. 
[22] Walter Rudin. Functional Analysis. McGraw-Hill, 1973.

[23] Aidan Sims. Hausdorff Étale Groupoids and Their $\mathrm{C}^{*}$-Algebras. arXiv preprint arXiv:1710.10897, 2017.

[24] Gerald Teschl. Topics in Real and Functional Analysis. unpublished, available online at http://www. mat. univie.ac.at/ ngerald/ftp/book-fa/fa.pdf, 1998.

[25] Klaus Thomsen. KMS States and Conformal Measures. Communications in Mathematical Physics, 316(3):615-640, 2012.

[26] Klaus Thomsen. Phase transition in $\mathrm{O}_{2}$. Communications in Mathematical Physics, 349(2):481-492, 2017.

[27] Dana P. Williams. Crossed Products of $\mathrm{C}^{*}$-Algebras. In Mathematical Surveys and Monographs, volume 134. American Mathematical Society, 2007. 